\title{
SOCIAL STRATIFICATION AND HEALTH
}

FOUR ESSAYS ON SOCIAL DETERMINANTS OF HEALTH AND WELLBEING

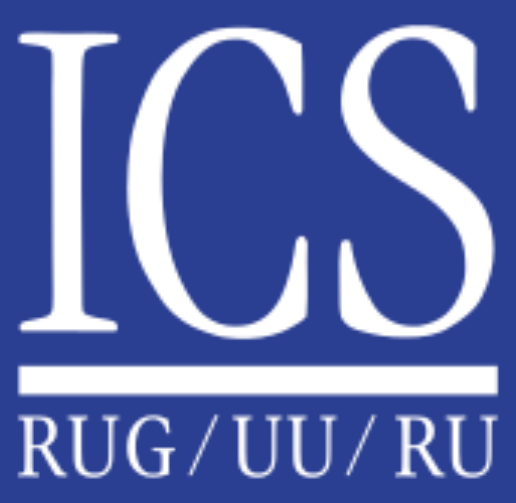





\section{Social Stratification and Health}

Four Essays on the Social Determinants

of Health and Wellbeing

Patrick Präg

ICS/Department of Sociology

University of Groningen 
To family and friends

ISBN: 978-94-6299-125-5

(C) Patrick Präg, 2015

Proofread by Julie Fricke

Printed by Ridderprint, 2015 
Patrick Präg: Social Stratification and Health. Four Essays on the Social Determinants of Health and Wellbeing

Thesis to obtain a doctoral degree at the University of Groningen, defended on July 2, 2015.

\section{Supervisors}

- Melinda Mills, ICS/University of Groningen

- Rafael Wittek, ICS/University of Groningen

\section{Assessment Committee}

· Jason Beckfield, Harvard University

- Ute Bültmann, University Medical Center Groningen

- Gerbert Kraaykamp, ICS/Radboud University Nijmegen

\section{The four essays of this thesis were published as}

· Präg, Patrick, and S.V. Subramanian. 2017. "Educational Inequalities in Self-Rated Health across US States and European Countries." International Journal of Public Health 62(6):709-716. doi: 10.1007/s00038-017-0981-6

• Präg, Patrick, Melinda Mills, and Rafael Wittek. 2016. "Subjective Socioeconomic Status and Health in Cross-National Comparison." Social Science Es Medicine 149:84-92. doi: 10.1016/j.socscimed.2015.11.044

- Präg, Patrick, Melinda Mills, and Rafael Wittek. 2014. "Income and Income Inequality as Social Determinants of Health: Do Social Comparisons Play a Role?" European Sociological Review 30(2):218-229. doi: 10.1093/esr/jct035

- Präg, Patrick, Rafael Wittek, and Melinda Mills. 2017. "The Educational Gradient in Self-Rated Health in Europe: Does the Doctor-Patient Relationship Make a Difference?" Acta Sociologica 60(4):325-341. doi: $10.1177 / 0001699316670715$ 



\section{Contents}

List of Tables $\quad$ ix

List of Figures

Acknowledgements xiii

1 Introduction $\quad 1$

1.1 Research problem and background . . . . . . . . . . . . . 1

1.1.1 Social determinants of health . . . . . . . . . . . . . 2

1.1.2 The social gradient in health . . . . . . . . . . . . . 2

1.1.3 Classical explanatory approaches ... . . . . . . . . . . . 3

1.2 Theoretical approaches and mechanisms . . . . . . . . . . . . . 4

1.2.1 Multidimensionality of SES . . . . . . . . . . . . 4

1.2.2 Understanding SES on different levels . . . . . . . . . . . . 6

1.3 Aim of this book and research questions . . . . . . . . . . . 9

1.4 Overview of the four studies . . . . . . . . . . . . . . . . . 12

1.4.1 Education-related health inequalities between US states in an international comparison . . . . . . . . . . . 12

1.4.2 Subjective SES and health in an international comparison . 13

1.4.3 Income inequality, income comparisons, and health . . . . . 13

1.4.4 The doctor-patient relationship and health . . . . . . . . . . 13

1.5 Data and analytic strategy . . . . . . . . . . . . . . . 14

1.5.1 Data . . . . . . . . . . . . . . . . 14

1.5.2 Analytic strategy . . . . . . . . . . . . . . 15

2 Education-Related Health Inequalities in US States in an Inter$\begin{array}{lr}\text { national Comparison } & 19\end{array}$

2.1 Introduction and background . . . . . . . . . . . . . . 19

2.2 Data and method . . . . . . . . . . . . . . . . . . . 21

2.3 Results . . . . . . . . . . . . . . . . . . . 23

2.4 Discussion . . . . . . . . . . . . . . . . . 25 


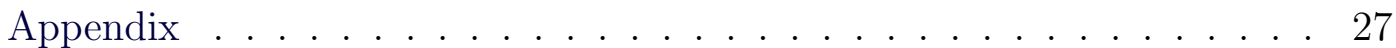

3 Subjective Socioeconomic Status and Health in a Cross-National $\begin{array}{lr}\text { Comparison } & 29\end{array}$

3.1 Introduction . . . . . . . . . . . . . . . . . . . . . . . . . . 29

3.2 Theoretical background . . . . . . . . . . . . . . . . . 31

3.2.1 The relationship between objective and subjective socioeconomic status $(\mathrm{SES}) \ldots \ldots . \ldots . \ldots . . \ldots 31$

3.2.2 The subjective SES-health relationship . . . . . . . . . . . . 32

3.2.3 Country affluence, income inequality, and the subjective SEShealth relationship . . . . . . . . . . . 33

3.3 Data and method . . . . . . . . . . . . . . . . . 35

3.3 .1 Data . . . . . . . . . . . . . . . . 35

3.3.2 Method and modeling strategy . . . . . . . . . . . 38

3.4 Results . . . . . . . . . . . . . . . . . . . . . . 39

3.4.1 Descriptive findings . . . . . . . . . . . . . . . . . 39

3.4 .2 Multivariate analyses . . . . . . . . . . . . . . . . 41

3.4.3 Sensitivity analyses . . . . . . . . . . . . . . . 47

3.5 Discussion . . . . . . . . . . . . . . . . . . 50

Appendix . . . . . . . . . . . . . . . . . 51

4 Income and Income Inequality as Social Determinants of Health: $\begin{array}{ll}\text { Do Social Comparisons Play a Role? } & 57\end{array}$

4.1 Introduction . . . . . . . . . . . . . . . . . . . 57

4.2 Theoretical background . . . . . . . . . . . . . . . 60

4.2.1 The socioeconomic gradient in health . . . . . . . . . 60

4.2.2 The negative income inequality-health correlation . . . . . . 61

4.2.3 Social comparisons and psychobiological reactions to hierarchies ......................... 62

4.3 Data and methods . . . . . . . . . . . . . . . 64

4.3 .1 Data . . . . . . . . . . . . . . . 64

4.3 .2 Outcome variables . . . . . . . . . . . . . . 65

4.3.3 Predictor variables . . . . . . . . . . . . . . . 66

4.3.4 Method and modeling strategy . . . . . . . . . . . 68

4.4 Results . . . . . . . . . . . . . . . . . . . . . . 68

4.4.1 Main findings . . . . . . . . . . . . . . 68

4.4.2 Sensitivity analyses . . . . . . . . . . . . . . 71

4.5 Discussion . . . . . . . . . . . . . . . . . . . 71

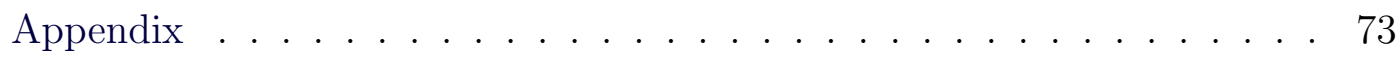


5 The Educational Gradient in Self-Rated Health in Europe: Does the Doctor-Patient Relationship Make a Difference? 93

5.1 Introduction . . . . . . . . . . . . . . . . . . . . . . 93

5.2 Theoretical background . . . . . . . . . . . . . . . . . 95

5.2.1 The doctor-patient relationship . . . . . . . . . . . . . 95

5.2.2 Paternalistic and egalitarian models . . . . . . . . . . . . 96

5.2.3 Socioeconomic status and doctor-patient relations . . . . . . 98

5.3 Data and methods . . . . . . . . . . . . . . . . . . . . . 99

5.3 .1 Data . . . . . . . . . . . . . . . . . . 99

5.3.2 Method and modeling strategy . . . . . . . . . . . . 102

5.4 Results . . . . . . . . . . . . . . . . . . . . . . . . 102

5.4 .1 Sensitivity analyses . . . . . . . . . . . . . . . 108

5.5 Discussion . . . . . . . . . . . . . . . . . . . . . . . . 108

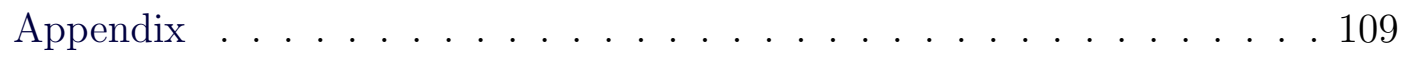

6 Conclusion 113

6.1 Summary of main findings . . . . . . . . . . . . . . . . . . 114

6.1.1 Educational health gradients in the US and Europe . . . . . 114

6.1.2 Subjective SES-health gradients in comparative perspective 114

6.1.3 Income comparisons, income inequality, and health . . . . . 114

6.1.4 The doctor-patient relationship and the social gradient in health . . . . . . . . . . . . . . . 115

6.2 Discussion and implications . . . . . . . . . . . . . . . . 116

6.3 Contributions . . . . . . . . . . . . . . . . . . 117

6.4 Limitations and directions for future research . . . . . . . . . . 118

$\begin{array}{lr}\text { Bibliography } & 121\end{array}$

$\begin{array}{lr}\text { Dutch summary } & 147\end{array}$

$\begin{array}{lr}\text { Curriculum Vitae } & 151\end{array}$

$\begin{array}{ll}\text { ICS Dissertation Series } & 153\end{array}$ 



\section{List of Tables}

1.1 Datasets used in the studies . . . . . . . . . . . . 15

2.1 Descriptive statistics by sample . . . . . . . . . . . . . 23

A2.1 Country and US state codes and sample sizes . . . . . . . . . 27

A2.1 Country and US state codes and sample sizes (cont'd) . . . . . 28

3.1 Descriptive statistics of individual-level variables . . . . . . . . 41

3.2 Self-rated health and psychological wellbeing regressed on several predictors (random coefficient models) . . . . . . . . . 43

3.3 Self-rated health and psychological wellbeing regressed on several predictors (random coefficient models), cross-level interactions . . . . . . . . . . . . . . . . . . 48

A3.1 Descriptive statistics of country-level variables . . . . . . . . 52

A3.2 Self-rated health regressed on several predictors excluding the French sample . . . . . . . . . . . . . . . 56

4.1 Descriptive statistics of individual-level variables . . . . . . . . 66

4.2 Psychological wellbeing and self-rated health regressed on several predictors (random intercept models) . . . . . . . . . 70

A4.1 Descriptive statistics of country-level variables . . . . . . . . . 74

A4.2 Psychological wellbeing and self-rated health regressed on several predictors (random intercept models), no upper age limit . 76

A4.3 Psychological wellbeing and self-rated health regressed on several predictors (random intercept models), excluding respondents older than $65 \ldots \ldots \ldots 77$

A4.4 Psychological wellbeing and self-rated health regressed on several predictors (random intercept models), excluding respondents older than $60 \ldots \ldots \ldots$. . . . . . . . . . 78

A4.5 Psychological wellbeing and self-rated health regressed on several predictors (random intercept models), expanded partnership status . . . . . . . . . . . . . . . . . 80 
A4.6 Psychological wellbeing and self-rated health regressed on several predictors (random intercept models), dummy indicator for missing income . . . . . . . . . . . . . . . . 82

A4.7 Psychological wellbeing and self-rated health regressed on several predictors (random intercept models), categorized Gini . . 84

A4.8 Psychological wellbeing and self-rated health regressed on several predictors (random slope models), random slopes for various predictors . . . . . . . . . . . . . . . . 86

A4.9 Psychological wellbeing and self-rated health regressed on several predictors (random slope models), random slope for income quintiles . . . . . . . . . . . . . . . . 88

A4.10 Psychological wellbeing and self-rated health regressed on several predictors (random intercept models), Gini averaged over several years . . . . . . . . . . . . . . . . 89

A4.11 Psychological wellbeing and self-rated health regressed on several predictors (random intercept models), three-way interaction 91

$5.1 \quad$ Descriptive statistics _. . . . . . . . . . . 100

5.2 Paternalistic and egalitarian doctor-patient relationship as measured in the second round of the ESS . . . . . . . . . . . . . . 101

5.3 Self-rated health regressed on education and doctor-patient relationship, OLS regression . . . . . . . . . . . . 103

5.4 Self-rated health regressed on education and doctor-patient relationship including interaction terms, OLS regression . . . . 106

A5.1 Self-rated health regressed on education and doctor-patient relationship, random intercept models . . . . . . . . . . . . . 110

A5.2 Self-rated health regressed on education and doctor-patient relationship including interaction terms, random intercept models111 


\section{List of Figures}

2.1 Prevalence rate, rate difference, and rate ratio of low and high educated Americans and Europeans . . . . . . . . . . . . . . . . 25

3.1 Average subjective SES by country . . . . . . . . . . . . . . . 39

3.2 Country-level correlations between self-rated health, psychological wellbeing, and subjective SES . . . . . . . . . . . . . . . 40

3.3 Slope variation of subjective SES and health outcomes . . . . . . . 44

3.4 Cross-level interaction plot for GDP per capita, subjective SES, and self-rated health . . . . . . . . . . . . . . . . . . . . 49

A3.1 Slope variation of subjective SES for self-rated health and psychological wellbeing, based on country-specific OLS models . . . . . . 53

A3.2 Scatterplots of subjective SES-health associations against GDP per capita and income inequality, based on country-specific OLS models 55

4.1 Average income comparison orientation by income and country . . . 67

5.1 Educational gradient in health by country, before and after adjusting for egalitarian doctor-patient relationship . . . . . . . . . . . . 104

5.2 Educational gradient in health by country, before and after adjusting for egalitarian doctor-patient relationship . . . . . . . . . . . 107 



\section{Acknowledgements}

Quite a number of people deserve thanks for their support while I completed this project. First of all, I would like to thank my supervisors Rafael Wittek and Melinda Mills for their encouragement and help. I thank SV Subramanian, also for hosting my stay at the Harvard School of Public Health and for co-authoring one of the chapters of this book. I am grateful to the members of the reading committee Jason Beckfield, Ute Bültmann, and Gerbert Kraaykamp for taking the time to read and comment on the present thesis.

Furthermore, I think that this is also a good opportunity to thank those who have supervised my work or who have collaborated with me in the past years or at the present; specifically, Barbara Beham, Sonja Drobnič, Lea Ellwardt, Dirk Enzmann, Eva Kibele, Sascha Peter, and Roland Verwiebe. I have learned a lot from them and I am looking forward to continuing to do so in the future.

This work also would not have been possible without the vibrant and supportive atmosphere at the Department of Sociology in Groningen and at the Interuniversity Center for Social Science Theory and Methodology (ICS). Special thanks go to Rie Bosman and Marijtje van Duijn. The members of the former Inequality in the Life Course research group in Groningen, among them Sissi Balbo, Katia Begall, Maja Djundeva, and Felix Tropf, deserve the biggest praise for interesting discussions and great insights. I am grateful that Maja Djundeva and Tina Kretschmer were willing to serve as paranymphs for the dissertation defense.

Thanks are also due to Miranda Visser, who copyedited the Dutch summary. Yes, you read that correctly: I also wrote the Dutch summary myself. 



\section{Chapter 1}

\section{Introduction}

\subsection{Research problem and background}

A large body of research has established a robust relationship between social stratification and a large variety of health outcomes (House, 2002; Elo, 2009; Adler et al., 1994; Kitagawa and Hauser, 1973; Olshansky et al., 2012). Those with better jobs, higher incomes, better education, higher status, and living in wealthier areas live longer and healthier lives. ${ }^{1}$ Nonetheless, important questions remain, and the most prominent one referring to the precise reasons underlying this relationship (Adler et al., 1994; Elo, 2009; Hedström and Swedberg, 1996). A well-known impediment to addressing this question properly is the multifaceted nature of socioeconomic status. While some researchers have attempted to map individuals onto one-dimensional, catch-all measures of socioeconomic status (Liberatos et al., 1988), social stratification research has reached a consensus that SES is a complex phenomenon that cannot be captured by a single variable (Braveman et al., 2005; Chen et al., 2013; Krieger et al., 1997; Torssander and Erikson, 2010). Furthermore, the multifaceted nature of SES incorporates both objective and subjective aspects that determine one's health. It is not only the material aspects of SES that affect health, such as economic resources and living conditions, but also the subjective appraisal of these material conditions. In the Chapters that follow, we present a multifaceted approach towards socioeconomic status with the goal of shedding light on the mechanisms linking SES and health. A crucial distinction between different facets of socioeconomic status is the one between its objective and subjective components (Schnittker and McLeod, 2005), which will guide our exploration in this book.

\footnotetext{
${ }^{1}$ Other dimensions of social stratification, such as sex, race, or migrant status are interesting as well but beyond the scope of this book.
} 


\subsubsection{Social determinants of health}

Research on health and illness has long been the principal domain of the medical sciences, studying the biological and genetic processes causing diseases. This singular focus on the biomedical paradigm in research on health, however, has long been challenged by the social sciences (Bloom, 2002). Research has been able to show that social contacts (Berkman and Syme, 1979; Holt-Lunstad et al., 2010), material living conditions (McKeown, 1979), and socioeconomic status (Townsend and Davidson, 1982) are major determinants of health. While these findings might not be surprising for sociologists - one of the first sociological studies found that those who are less well socially integrated are more likely to commit suicide (Durkheim, 1951) - , they helped putting the role of biological and genetic approaches into perspective: social factors must not be neglected in the study of health and illness. Moreover, social determinants of health have gained in importance, as infectious diseases have become less important as the main causes of death. Chronicincurable, long-term - diseases are nowadays the most crucial threats to health and well-being. Against the backdrop of aging societies, social factors will further gain in importance, also for aging healthily and happily (Stolk et al., 2009).

\subsubsection{The social gradient in health}

Sociological research on health long relied on the assumption that socioeconomic status was linked to health only in the form of poverty (Adler and Ostrove, 1999). Outside of poverty research, SES was mostly seen as a nuisance factor in research that needed to be controlled for statistically. But since the 1980's, this assumption was challenged, probably most forcefully when Marmot et al. $(1978,1984)$ showed an increase of cardiovascular disease and mortality risk with each decrease in occupational grade in the Whitehall Study. Given the fact that the sample of the Whitehall study solely consisted of British civil servants, poverty could clearly be ruled out as a cause for the social gradient. This finding was replicated for a variety of populations and health outcomes, giving rise to a vast line of research on the social gradient in health (Adler and Rehkopf, 2008). This doesn't just hold true for individuals living in contemporary societies with free and and extensive public health care coverage (Elo, 2009; Mackenbach et al., 2008); historical evidence also documents that there was a social gradient in health in $17^{\text {th }}$ century Geneva (Whitehead, 1997), as well as in ancient Greece, Egypt, and China (Krieger et al., 1997). ${ }^{2}$

\footnotetext{
${ }^{2}$ But also see e.g. Harris (2004) or Bengtsson and Van Poppel (2011) for evidence of the historical variation in social gradients in health.
} 


\subsubsection{Classical explanatory approaches}

Given the robustness of the relationship between SES and health outcomes, it raises the question as to which factors would account for this relationship. A classical typology of reasons was drawn up by Townsend and Davidson (1982). Townsend and Davidson (1982) distinguished between four different types of explanations that are traditionally seen to be at the core of the social gradient in health. 1) Artifact explanations refute the existence of a social gradient in health and attribute any such findings to spuriousness and measurement error. Although this explanatory approach has not been able to rule out the existence of a social gradient in health, it has great merits in highlighting the numerous methodological problems that arise when researching health inequalities, such as the lack of a consensus on how to measure SES properly (e.g. Chandola, 1998; Torssander and Erikson, 2010). 2) Social selection explanations suggest that the connection between SES and health is essentially a case of selection bias. Bad health induces socioeconomic downward mobility, thus individuals with poor health are selected into worse socioeconomic positions. Whereas this explanation is certainly part of the story of the social gradient in health, longitudinal studies have shown that selection effects are much smaller than the effects of SES on health (Chandola et al., 2003; Thiede and Traub, 1997). 3) The cultural/behavioral explanation points to lifestyle factors, such as smoking, poor diet, and lack of exercise, as underlying reasons of health inequalities. Research shows that these lifestyle factors are unevenly distributed across social strata and play a major role in the generation of the social gradient (Pampel et al., 2010). 4) Structural/materialist explanations identify the manifest physical living conditions of individuals as causes of the social gradient in health. Living conditions, such as housing and working conditions, clearly have an impact on physical health (e.g. Aldabe et al., 2011), and large SES differentials in material living conditions can be found (Präg et al., 2010).

Apart from these four classical explanatory approaches, two additional approaches have been developed in the past years (Macintyre, 1997; Nettleton, 2006). 5) The life course approach focuses on trajectories of cumulative health (dis)advantage (Kuh and Ben-Shlomo, 2004; Willson et al., 2007; Pavalko and Caputo, 2013), where health is conceptualized as a life course capital that is preserved or depleted over time. 6) Psychosocial factors have also been suggested as major predictors for the SES-health relationship. For instance, anxiety and stress, resulting from unfavorable social comparisons and lack of social support, have been suggested to increase inequality in health (Marmot, 2004; Schnittker and McLeod, 2005; Wilkinson and Pickett, 2010). 


\subsection{Theoretical approaches and mechanisms}

Our analyses in this book revolve around two interlinked aspects of the social gradient in health. On the one hand, we will explore different dimensions comprising SES, specifically the tension between its objective and subjective aspects. On the other hand, social stratification also affects health on different levels, not only on the level of the individual and the household, but also on the levels of regions and societies one is living in.

\subsubsection{Multidimensionality of SES}

Social stratification research typically distinguishes between several dimensions of SES, most prominently education, income, and occupation-related measures, such as occupational prestige or class (Lahelma, 2001). These dimensions of SES can group individuals, households, and societies in a vertical fashion, with those with low education, low income, or low prestige towards the bottom of a hierarchy, and those with high education, income, or prestige at the top. While there is substantial overlap between the different dimensions, the heterogeneity between the dimensions can be crucial (Braveman et al., 2005; Geyer et al., 2006; Lenski, 1954; Jackson, 1962). For instance, some individuals can have remarkable work careers and achieve high occupational prestige, while having only little education, or they can have attained a high educational degree, while failing to obtain a job that provide them a high income.

Objective measures of SES have been shown to be related to important health outcomes. Seminal studies and meta-analyses have shown substantial disparities with respect to mortality and other health outcomes, specifically between educational groups, occupations, and income groups (Kitagawa and Hauser, 1973; Furnée et al., 2008; Kunst et al., 1998; Mirowsky and Ross, 2003). Different mechanisms have been discussed in the context of these relationships (Adler and Rehkopf, 2008), among them health literacy and self-management ability for education, adverse and dangerous working conditions for occupational groups, and differential access to resources for income groups. However, studies have suggested that these mechanisms cannot account for all of the SES differences that are found in health.

A classical objective indicator of SES is education. It mainly draws on the knowledge-based resources on an individual, but captures other aspects, such as life chances as well. A large number of studies have shown the beneficial health effects of education (e.g. Lleras-Muney, 2005; Masters et al., 2012; Mirowsky and Ross, 2003). Factors linking education and health are less financial hardship and better employment possibilities (Ross and Wu, 1996), greater command of social- 
psychological resources, such as social contacts (Ellwardt et al., 2014; McPherson et al., 2006), greater sense of control (Mirowsky and Ross, 2003), less stress and fewer traumatic life events (Lantz et al., 2005), and better health behaviors (Pampel et al., 2010). Furthermore, it reflects the transition from parents' SES and one's own adulthood SES, capturing both the material, cognitive, and other resources available from the family background, as well as adult resources that affect health (Galobardes et al., 2006). Education also affects the level of knowledge, skills, and what has been termed 'health literacy' (Williams et al., 1995) of individuals. Previous studies have suggested that the strength of the relationship between education and health varies across countries (e.g. Eikemo et al., 2008a; Von dem Knesebeck et al., 2006); however, these have largely overlooked the variation in the size of the education-health gradient.

Income is another major objective indicator of SES. Income is a direct measure of the material resources component of SES. While money in and of itself is unlikely to be a direct determinant of health and illness, it facilitates obtaining goods and services that can enhance and maintain health. Furthermore, income can also affect health via non-material pathways, for instance by inducing feelings of relative deprivation among those with low income (Huijts et al., 2010a).

One dimension of SES that has gained recent prominence, especially in research on public health, is subjective socioeconomic status (Adler et al., 2000; Adler, 2013; Cohen et al., 2008). For measuring subjective SES, individuals are typically asked to locate themselves in the social hierarchy, rather than relying on objective measures, such as education, income, or occupation. Furthermore, the relationship between subjective SES and health (Adler et al., 2000; Nobles et al., 2013) is usually found over and above the effects of objective SES markers. This has been interpreted as the detrimental health effects of status differentials (SinghManoux et al., 2005). Individuals perceiving themselves to be low in social status exhibit worse health via a direct pathway, namely via neuroendocrinological stress reactions, and via an indirect pathway, namely poor health behaviors, such as smoking or overeating, which serve as coping reactions to the perceived lower status position.

One of the major problems of the research that tries to disentangle the health effects of subjective SES is the difficulty of distinguishing the status effects, as measured by subjective SES (while controlling for objective SES), from any measurement imprecision the objective SES measures might have. Subjective SES has been praised for its ability to capture facets of objective SES, such as parental background, SES of the partner, or inherited wealth, that the traditional measures of occupational prestige, education, and income arguably only poorly reflect. For this reason, using a more direct measure of status differences can overcome 
this problem. A crucial aspect of the status explanation of health inequalities is the self-location of individuals in the social hierarchy. Research has shown that individuals vary in their propensity to engage in such status comparisons, and we posit that individuals which attribute a higher importance to status comparisons should also experience more pronounced health effects of their status position. Therefore, investigating the health effects of income comparisons in relationship to their income position in the national income hierarchy can yield important insights.

An additional dimension of SES inequalities in health is their relational aspect. Power is an understudied dimension in social stratification research. A look at the associations of different styles of doctor-patient communication with health outcomes and how these associations vary by educational groups can shed light into this dimension of health inequalities. Over the past decades, the prevailing paternalistic style of doctor-patient communication, which largely relied on the deference of the patient to the authority of the doctor, has shifted towards a more egalitarian model, where patients are accepted as experts of their own symptoms, doctors offer patients choices between treatment options, and consensual decisions on treatments are sought.

\subsubsection{Understanding SES on different levels}

Research on health inequalities focuses on two different areas of interest. One area is concerned with what has been called "downstream" factors, namely the mechanisms concerned with how the experience of social life "get under the skin." Research in this field is concerned with the effects of stress and coping (Dickerson and Kemeny, 2004; Thoits, 2010), health behaviors (Pampel et al., 2010; Umberson et al., 2010), and the physiological processes related to them (e.g. Epel et al., 2004; McEwen, 1998). Another area of research is occupied with the so-called 'upstream' processes (Braveman et al., 2011) such as national policy arrangements (Bambra, 2011; Beckfield and Krieger, 2009; Brennenstuhl et al., 2012; Navarro et al., 2006; Olafsdottir and Beckfield, 2011), in particular health care systems (Beckfield et al., 2013; Wendt et al., 2009).

Despite the fact that these two types of processes should best be seen in conjunction to get a full picture of the social determinants of health, there is often little explicit interconnection between the two in the existing research. Of course, research on downstream factors acknowledges the important role of the social forces shaping the downstream processes, but only little explicit attention is given to the structural origins of the downstream processes in empirical studies and any modifying effects of social contexts on downstream processes are largely 
neglected (Schnittker and McLeod, 2005). In research on upstream determinants of health, links between the upstream processes and health outcomes on the microlevel, especially in a cross-national context (Olafsdottir, 2007), are rarely explored. Strengthening the links between upstream and downstream processes, and understanding the interactions between the two, will increase our knowledge of the social processes that determine health and wellbeing. In order to better link the upstream and downstream processes, a multilevel approach is warranted.

Health differences by SES occur in social contexts. Not only the individual SES position of an individual affects their health, but also aspects of the context they live in. An especially salient context is the overall society in which they live, however, other contexts should not be neglected.

\section{The "comparative turn" in health inequalities research}

The growing importance of comparisons across societies (Mills et al., 2006) has also recently started to affect research on health inequalities. There is a longstanding tradition of comparing population health disparities between countries (e.g. Deaton, 2013), but only recently health disparities within countries have taken the center stage in cross-national comparisons (e.g. Mackenbach et al., 2008, 2015a,b; Olafsdottir and Beckfield, 2011). While public health research in general has long been interested in the effects of 'upstream,' institutional factors driving health outcomes (Beckfield and Krieger, 2009; Beckfield et al., 2013), research on health inequalities has experienced what can be called a 'comparative turn' (Olafsdottir et al., 2013). Comparing the extent of health inequalities across countries allows us to put them into context and gives a fresh impression of which inequalities are considered to be 'large' or 'small.' Furthermore, comparing health inequalities across societies allows a more systematic examination of contextual factors, such as income inequality or welfare state policies, that can affect the size of socioeconomic gradients in health. By doing so, comparative health inequalities research can potentially give researchers and policy makers a sense of the malleability of health inequalities.

\section{The health effects of income inequality}

The relationship of income inequality in a country and population health is a crucial public health debate taking place not only in academia, but also in the media and among policy makers. The debate originally emerged from public health research (Rodgers, 1979; Wilkinson, 1992, 1996; Wilkinson and Pickett, 2010), but has also had strong repercussions in sociological social stratification research (Beckfield, 2004; Wilkinson and Pickett, 2009). Next to the individual variation in SES, 
income inequality has been suggested to affect population health. Specifically, social inequalities in a country have been suggested to reduce the health of the population of that country. While early in the debate, sociologists have dismissed this public health research for reasons of poor theoretical elaboration, faulty operationalization, and doubts about causality (e.g. Beckfield, 2004; Goldthorpe, 2010), more recently it has been argued that many questions raised in this debate are genuinely sociological and should be addressed by sociologists (Layte and Whelan, 2014).

The debate revolves around two major issues. Firstly, the existence and the causality of an association between income inequality and health is questioned. Secondly, assuming that there is a causal association between the two, the mechanisms which link income inequality and population health are disputed. Although Beckfield (2004) cast doubts on the existence of a causal effect of income inequality by showing that any negative effect of income inequality was spurious due to unobserved country characteristics in a rigorous fixed-effect examination of a large-country panel, a recent replication with a longer observation period and a larger number of countries did find negative income inequality effects in some groups of countries even after accounting for such unobserved characteristics (Pop et al., 2013). A large meta-analysis of existing multilevel studies, which can also account for population composition, hinted at the presence of a negative effect of income inequality on health (Kondo et al., 2009). Also, a recent review paper disputes claims that the inequality-health correlation is only incidental (Pickett and Wilkinson, 2015).

Those researchers who have found an effect of income inequality on population health have suggested several pathways which underlie this relationship (Layte, 2012). A first option is social trust. In a nutshell, the underlying mechanism for this pathway is that greater economic inequality in a society erodes social trust due to fewer possibilities for homophilous ties, thus making social support, an important factor affecting health, more difficult (Ellwardt et al., 2014). A second option is the neo-materialistic pathway, which suggests that income inequality is an indicator of underinvestment into societal resources, both in the sense of infrastructure like social services and in the sense of human capital, which then, in turn, is detrimental for population health (Lynch et al., 2000). A third, not necessarily mutually exclusive pathway, is status considerations, which lead to greater anxiety with respect to social prestige in societies with greater economic inequality (Layte and Whelan, 2014), and thus affect health negatively.

Furthermore, it has been suggested that income inequality also affects the size of health gradients in countries (Beckfield et al., 2013; Semyonov et al., 2013; Wilkinson and Pickett, 2008). Different pathways have been suggested for this. 
High-SES invididuals in less egalitarian societies might have more resources that they can translate more easily into better health, leaving the disadvantaged even further behind in terms of health (Beckfield et al., 2013). Alternatively, societies with a high degree of income inequality are characterized by a country's systematic underinvestment across a wide range of human, physical, and social infrastructures. The less well-off are likely to suffer most from these underinvestments, as they lack the personal resources to make up for these public underinvestments; thus, health inequalities in countries with greater income inequality could be greater (Semyonov et al., 2013). Finally, Wilkinson and Pickett (2008) suggest that status competition should be stronger in places characterized by greater income inequality, thus exacerbating health inequalities via a status differentiation pathway.

\subsection{Aim of this book and research questions}

What seems to play less of a role in the traditional approaches to explain the SES-health relationship are two factors: firstly, the multifaceted nature of SES, and secondly, that SES can exert its effects on different levels. The multifaceted nature of SES refers to the fact that SES not only manifests itself in objective, easily observable indicators, such as education, income, and occupation, but also in subjective indicators (to which less attention has been paid) that are assumed to be important drivers of health inequalities. While it has long been assumed that SES is largely active on the individual level, recent research has gathered evidence that SES also affects health on multiple levels.

In this book, we will explore various crucial aspects of the relationship between SES and health. In a first step, we will look at the variation across countries in the size of the relationship between one traditional aspect of SES, namely education, and self-reported poor health.

- How does the size of the relationship between education and health vary across different spatial entities, such as countries and sub-national regions? (Chapter 2)

Answering this question will allow us to get a better understanding of the malleability of the social gradient in health. In a (hypothetical) world where the relationship between SES and health comes down to biological or genetic determinism, we would not expect any variation in the size of the SES-health gradient across different spatial units. However, being able to see the variation in the size of the SES-health gradient across places illustrates the relevance of the social forces shaping health and the potential for policy intervention to reduce health inequalities. 
At the same time, however, one should not be mistaken about the assumed within-country homogeneity when comparing the sizes of health gradients across countries. Data allow us to break down the education-health relationship by subnational units in one particularly interesting country. In the United States, we are able to compare the SES-health relationship across the 50 federal states. The US is an intriguing case due to the discrepancy between its great wealth and, at the same time, its poor population health performance when compared to similar high-income countries (e.g. Avendano and Kawachi, 2014). Furthermore, the US is notorious for its vast heterogeneity in terms of population health across its regions (e.g Murray et al., 2006).

Therefore, exploring the within-US heterogeneity in the education-health relationship will provide us with potentially important insights into the social stratification of health in the US:

- How does the size of the relationship between education and health within the US compare to the same relationship in European countries? (Chapter 2)

We will then move on to the subjective mechanisms linking SES and health. In Chapter 3, we will focus on a relatively novel SES marker in research on health inequalities. Subjective SES is seen as a self-appraisal about one's own location in a socioeconomic status order and directly touches upon the perceptional aspects of social stratification. Given that previous research has shown that subjective SES is a powerful predictor of health in a variety of settings, we are interested in seeing whether this finding also holds in a cross-national comparison. Furthermore, previous research has also established that the subjective SES-health relationship remains substantially important even after accounting for objective markers of SES.

- Does the subjective SES-health relationship replicate across countries? (Chapter 3 )

- Does the subjective SES-health relationship replicate across countries, even after controlling for objective markers of SES? (Chapter 3)

Furthermore, exploring these questions in a cross-national setting allows us to go one step beyond and to explore the variation in the size of the subjective SEShealth relationship as well as to attempt to identify societal-level factors that can shed light onto the processes generating this variability. Country affluence and income inequality play important roles in the debate about societal-level determinants of population health and can be assumed to affect variation in the subjective SES-health relationship. Thus, we also pose the following questions: 
- Is there variation in the SES-health relationship across countries? (Chapter 3 )

- Can the variation in the SES-health relationship across countries be explained by differences in national income or income inequality in a country? (Chapter 3)

We will continue to explore the links between income, income inequality, and health. The previous literature has suggested that status competition is an important mechanism linking relative income and income inequality to population health. One promising approach to test this pathway is to exploit the variation in the importance individuals attribute to comparing their incomes to those of others. Individuals vary in their orientation towards social comparisons, and we would expect that individuals with a stronger social comparison orientation should experience greater negative health effects of higher income inequality and lower relative income.

- Is the relationship between income inequality and health moderated by one's income comparison orientation? (Chapter 4)

- Is the relationship between relative income and health moderated by one's income comparison orientation? (Chapter 4)

Furthermore, another important aspect of SES is its relational dimension which is relatively rarely explored. This dimension can be expected to strongly play out in the health domain when it comes to the relationship between doctors and their patients. When it comes to health advice and treatment decisions, doctors possess great authority and power over their patients. While the doctor-patient relation in the past was largely built on this asymmetry, fostering a paternalistic or doctor-centered model of the relationships between doctors and patients, various developments in society and the health domain have given rise to a more egalitarian or patient-centered model of doctor-patient relations, aiming for joint decisionmaking and treating their patients as experts for their own symptoms and their own living situation. The question arises how such variation in the doctor-patient relationship affect social inequalities in health.

- How is the doctor-patient relationship associated with the SES gradient in health? (Chapter 5) 


\subsection{Overview of the four studies}

This selection of studies presented in this book replicates and extends existing research in several major ways. Firstly, we explore the variation in the strength of the education-health association not only across countries, but also within a country with marked health and socioeconomic inequalities: the United States. By doing so, we gain greater insight into the variability and malleability of educationrelated health inequalities across and within countries. Whereas the relationship between SES and health is sometimes reduced to genetic and medical factors, acknowledging the educational differences not only between, but also within societies, highlights the importance of social, 'upstream' determinants of population health and points to potentials for policy intervention. Secondly, we examine the relationship between subjective SES and health in a cross-national comparison. While previous studies were able to show the association between subjective SES and health only in single societal contexts, we are able to show that the relationship between subjective SES and health holds in 27 countries from all over the world, even over and above the influences of objective status markers. Thirdly, we propose and empirically test a new link between income inequality and health, namely social comparison orientations. Social comparisons are assumed to be a key mechanism in the pathway between income inequality and health. Drawing on data from 23 European countries, we are able to show that a greater reported importance of reporting one's income does not exacerbate the negative effect of income inequality on health. Fourthly, we explore the association between the doctor-patient relationship and the educational health gradient across countries, adding a relational perspective to the research on SES and health.

\subsubsection{Education-related health inequalities between US sta- tes in an international comparison}

Chapter 2 focuses on a conventional and objective SES indicator, namely education, with the aim to explore variation in the size of the education-related health gradient comparing US states and European countries. The rationale for this approach is that comparative research on the SES - health relationship has recently focused on comparing European countries to the United States. What has been overlooked so far is within-US heterogeneity with respect to health gradients. As the US data we use for our analysis allow us to break down the analysis by the federal states of the US, we can compare the relationship in US states to those found in European countries. 


\subsubsection{Subjective SES and health in an international com- parison}

Chapter 3 departs from the key finding of recent research on health inequalities that there is substantial variation in the size of health gradients across countries. Cross-national studies have not only shown that objective markers of SES, such as income, education, and social class, are generally associated with health outcomes, but also that the sizes of these associations vary across countries. An SES indicator status that has only recently been introduced in research on health inequalities is subjective socioeconomic status. Studies revealed that subjective socioeconomic status is also associated with health, usually over and above the effects of objective SES markers. This evidence has been interpreted as proof of social status effects on health.

In our study, we aim to test the robustness of the association between subjective SES and health in a sample of nationally representative, standardized surveys from 27 countries worldwide. We will conduct multilevel analyses to explore whether the relationship between subjective SES and health holds when controlling for objective measures of SES, namely education, income, and occupational prestige. Additionally, we will test whether the cross-national variation in the subjective SES-health relationship is affected by national income and income inequality in a country.

\subsubsection{Income inequality, income comparisons, and health}

Chapter 4 tackles the income inequality-health relationship and the social gradient in health from a different angle. Both of these phenomena have been suggested to be explainable by the mechanism of status comparisons. This, however, has rarely ever been tested in a direct fashion. In this article, we explicate and test this mechanism by assessing the role of social comparison orientation. Research has shown that individuals vary in their propensity to engage in social comparisons, and those with a higher propensity are also more likely to be affected by the outcomes of such comparisons. In our analysis, we check whether the tendency to compare one's income to that of others can contribute to explaining socio-economic disparities in health.

\subsubsection{The doctor-patient relationship and health}

Chapter 5 addresses the relational aspects of the social gradient in health. Research suggests that doctor-patient relations have evolved from a doctor-centered, 
paternalistic approach towards a more patient-centered, egalitarian model of interactions between physicians and their patients. Given the long-running debate on the relationship between education and health, the question arises how differences in doctor-patient relations affects educational inequalities in health. First, we test whether a more egalitarian doctor-patient relationship is associated with a reduced educational gradient in self-reported health. Second, we test whether egalitarian (e.g. discussing treatment decisions with patients) and paternalistic (e.g. withholding some information from patients) doctor-patient relations show differential effects on self-reported health across different educational groups.

\subsection{Data and analytic strategy}

\subsubsection{Data}

In order to test our theoretical ideas, we make use of the five different datasets listed in Table 1.1. Major advantages of these data sets are the relatively extant coverage of countries and individuals, which facilitates cross-national comparisons. A slight disadvantage of the data sets are the availability of relatively few health markers, which are also based on self-reports only. Nonetheless, the data sets used provide a high data quality and a high degree of comparability due to tight quality controls (EVS and GESIS, 2011; Jowell et al., 2007).

For Chapter 2, we pool information from European Values Study (EVS; EVS and GESIS, 2011) and the Behavioral Risk Factor Surveillance System (BRFSS; CDC, 2008). The European Values Study provides the biggest number of European countries in a recent academically-driven population survey and includes a single item on self-reported health. Sample sizes in the individual countries are on average $N=1,100$. The Behavioral Risk Factor Surveillance System is a US-based survey that comprises ample health indicators, among them a measure of self-reported health. The major advantage of the BRFSS is the large sample size in the federal states (average $N=5,400$ ), which allow us to break down the data by federal states and to explore the variability in health inequalities between the US states.

In Chapter 3, we make use of the recently released International Social Survey Program (ISSP) 2011 module on "Health and Health Care" (ISSP Research Group, 2013). This data set provides us with information on health outcomes, such as selfrated health and psychological wellbeing, as well as our focal independent variable subjective SES.

In Chapters 4 and 5, we exploit the second and the third round of the European Social Survey (ESS, Jowell et al., 2007). An item on self-rated health is part of the core questionnaire of the ESS. In addition, the third round fielded a module on 
Table 1.1: Datasets used in the studies

\begin{tabular}{|c|c|c|c|c|}
\hline Data set & Reference & $\begin{array}{l}\text { Year data } \\
\text { collection }\end{array}$ & $\begin{array}{l}\text { No. of } \\
\text { coun- } \\
\text { tries }\end{array}$ & Chapter \\
\hline $\begin{array}{l}\text { Behavioral Risk Factor Surveillance } \\
\text { System (BRFSS) }\end{array}$ & CDC (2008) & 2008 & $51^{1}$ & Ch. 2 \\
\hline European Values Study (EVS) & $\begin{array}{l}\text { EVS and } \\
\text { GESIS } \\
(2011)\end{array}$ & 2008 & $45^{2}$ & Ch. 2 \\
\hline $\begin{array}{l}\text { International Social Survey Program } \\
\text { (ISSP) } 2011\end{array}$ & $\begin{array}{ll}\text { ISSP } & \text { Re- } \\
\text { search } & \\
\text { Group } & \\
(2013) & \end{array}$ & $2011-13$ & 27 & Ch. 3 \\
\hline European Social Survey (ESS) Round 3 & $\begin{array}{l}\text { Huppert } \\
\text { et al. }(2009)\end{array}$ & $2006 / 07$ & 23 & Ch. 4 \\
\hline European Social Survey (ESS) Round 2 & $\begin{array}{l}\text { Kooiker } \\
\text { et al. }(2004)\end{array}$ & $2004 / 05$ & 24 & Ch. 5 \\
\hline
\end{tabular}

Notes: ${ }^{1} 50$ federal states of the US and the District of Columbia,

${ }^{2} 44$ countries and the region of Kosovo

"Personal and Social Wellbeing" (third round, Huppert et al., 2009) which includes an item on the income comparison orientation of individuals. This will provide the data for the analyses conducted in Chapter 4. The second round included a module on "Opinions on Health and Care Seeking" (Kooiker et al., 2004), which provides information on opinions about the doctor-patient relationship. This will be the focus of Chapter 5 .

\subsubsection{Analytic strategy}

The studies presented in this book are standing in the tradition of cross-national research (Kohn, 1987; Mills et al., 2006), drawing on data from multiple countries and exploiting differences between the countries to better understand the social forces shaping the lives and, in particular, the health and wellbeing of individuals. Cross-national research typically deals with questions that extend across multiple levels. Major challenges in this context are being able to disentangle compositional and true contextual effects: Do countries differ in one way or the other because their populations differ in their composition (e.g. more older people, more highlyeducated people), or are the differences truly due to contextual factors (e.g. higher income inequality)? Another general question driving cross-national research is the interactions between processes located at the country level and those at the individual level, for instance, whether income inequality exacerbates the social gradients in health in countries.

Statistical methodology and, probably more importantly, increased computa- 
tional speed, have made answering such questions much easier in the past years. A major trend has been the proliferation of random intercept and random coefficient models in cross-national research (Kreft and De Leeuw, 1998; Snijders and Boskers, 2012; Subramanian et al., 2003). Stemming originally from educational research that focused on students clustered in classrooms, which were, in turn, clustered in schools, these models where applied to individuals nested in countries for the purposes of cross-national research.

Random effects models give researchers greater accuracy over standard OLS models when it comes to accounting for the clustering of individuals in countries. Furthermore, they are an extremely flexible approach to modeling cross-national data. However, researchers have recently started pointing out problems in standard applications of random effects models in cross-national research. On the one hand, researchers have pointed to problems of estimating the variance components correctly when the number of countries is low (Bell et al., 2014; Bryan and Jenkins, 2015). While the number of level-2 units in studies of classrooms and neighborhoods can easily be several dozen, the number of countries in a multilevel study is rarely above thirty and often less than 25. On the other hand, researchers coming from the tradition of panel data analysis are calling the random effects assumption underlying random effects models into question (Allison, 2009; Halaby, 2004).

In order to overcome these problems, researchers have pointed to the merits of what has been called two-step regression models (Kedar and Shively, 2005; Wooldridge, 2010). In this approach, single-level regression models are fitted to each country data set separately in the first step. In the second step, the parameters of interest are extracted from these models and then modeled as outcomes in a second single-level model.

Given the fact that the number of units on the lowest level, the level of individuals, is usually large, one of the advantages of random effects models, the partial pooling and borrowing strength property (Jones and Bullen, 1994), is of less importance. This property of random effects models can be crucial to increase the precision of level-1 parameters when the average number of level-1 units is low (such as students in classrooms), but in multi-country studies, where the number of individuals is usually in the thousands, it gives researchers little or no advantage over single-level models.

Given the fact that the jury is still out about who is ultimately right in these debates (and expecting that it is unlikely that one single approach turns out to be ultimately right), the Chapters of this book will use both strategies. In Chapter 2, where the focus is on exploring differences between level-2 units with large sample sizes, there is clearly nothing to be gained by using random effects models. Singlelevel models for each unit separately allow the removal of compositional effects from 
the predicted prevalence rates of poor health and also allow for a more intuitive prediction of these rates. In Chapters 3 to 4 , the analyses will largely draw on random effects models, which are, however, checked for their robustness in twostep regression models. In Chapter 5, both fixed and random effects models will be reported. 



\section{Chapter 2}

\section{Education-Related Health Inequalities in US States in an International Comparison $^{1}$}

\subsection{Introduction and background}

When compared to other industrialized, high-income countries, the US population exhibits a distinct health disadvantage across important health outcomes, such as infant mortality, heart disease, sexually transmitted diseases, injuries from violence and motor vehicle crashes, and life expectancy (Avendano and Kawachi, 2014; Bezruchka, 2012; Crimmins et al., 2011; US Burden of Disease Collaborators, 2013; Woolf and Aron, 2013). While differences in health care might be one cause of this disadvantage, it is unlikely that they are the main driver (Berkman and Epstein, 2008; Braveman et al., 2011): The US has the highest health care spending in the world (Thorpe et al., 2007; Woolf and Aron, 2013). Furthermore, the wide range of health indicators where the US is at a disadvantage underlines that medical care deficiencies cannot be the sole cause. A potential lever suggested to eliminate the overall health disadvantage of the US population is to reduce the health disadvantage of lower socioeconomic groups (Avendano et al., 2009; Avendano and Kawachi, 2014; Bayer et al., 2013; Dow and Rehkopf, 2010).

Various studies (Avendano et al., 2009, 2011; Avendano and Kawachi, 2011; Banks et al., 2006, 2009, 2010; Jürges, 2009; Kaplan et al., 2010; McDonough et al., 2010; Sacker et al., 2007; Semyonov et al., 2013; Woolf and Aron, 2013) have shown that the US as a whole indeed exhibits greater socioeconomic status (SES) inequalities in health than European countries. In addition, population health and health disparities in the US vary greatly by geographical location (Adler and

\footnotetext{
${ }^{1}$ This Chapter is co-authored with SV Subramanian.
} 
Rehkopf, 2008; Ezzati et al., 2008; Kindig and Cheng, 2013; Murray et al., 2005, 2006) and are not explainable by race or health care access and utilization alone (Avendano and Kawachi, 2014; Murray et al., 2006). In this study, we explore how within-US variation in the education-self-rated health association compares to the variation between European countries, taking both absolute and relative measures of health inequality into account.

We are aiming to shed light into these variations by looking into educational differences in self-rated health in 44 European countries and the 50 federal states of the US. By doing so, we attempt to overcome the 'methodological nationalism' (Mau and Verwiebe, 2010; Wimmer and Glick Schiller, 2002) which is prevalent in the majority of current comparative research on health inequalities. Researchers often rely on the nation-state as a seemingly 'natural' unit of analysis (Olafsdottir et al., 2013), but the focus on nation states as units of analyses may mask important heterogeneities within countries. By breaking down the US into its 50 federal states and comparing them to European countries, we take a first step toward looking into the black box of a nation-state in cross-national research, exploring the variance of health inequalities within and between countries. By focusing on the United States, and using European countries as a benchmark, we contrast two particularly interesting cases. Compared to Europe with its diverse and distinct historical trajectories and its resulting institutional variety (Mackenbach et al., 2013), the US have experienced a rather homogeneous development in terms of institutions $^{2}$, however, it still exhibits a well-known within-country heterogeneity in terms of health.

A second, distinct contribution of our paper is our focus on two separate indicators of health inequalities. The use of conceptually different measures of health inequalities has long hampered previous health disparities research. Health inequalities can both be conceived of as being relative - e.g. by using ratios of health outcomes in high- and low-SES groups - or as being absolute - by relying on differences between social groups (King et al., 2012; Mackenbach and Kunst, 1997). Valid arguments for using either of these metrics can be made, however, it has to be seen that both can yield different, sometimes even contradictory, results (Harper et al., 2010): In the case of a decreasing prevalence rate in the overall population, relative inequality is most likely to increase when the rate of decrease is somewhat higher for the better-off group than for the worse-off group, despite the fact that absolute inequality can be decreasing. In order to overcome this problem, our analyses will draw on absolute, as well as relative, measures of inequality

\footnotetext{
${ }^{2}$ While recent research has pointed to the legacy of Jim Crow laws for population health (Krieger et al., 2014; Subramanian et al., 2009), our focus on the non-Hispanic white population should ameliorate this concern.
} 
between educational groups in societies.

\subsection{Data and method}

We combine two data sets, the 2008 waves of the European Values Study (EVS) and the Behavioral Risk Factor Surveillance System (BRFSS). The fourth wave of the EVS (2011), collected in 2008, provides health self-ratings for samples of individuals from all countries of Europe with 100,000 or more inhabitants. Sampling and questionnaire translation procedures followed high quality standards to ensure comparability between countries. Methodological details about the survey can be found in EVS and GESIS (2011). Our European sample includes health information from 44 European countries and the region of Kosovo.

The Behavioral Risk Factor Surveillance System (CDC, 2008) is a US-state representative survey of the adult population living in households. The BRFSS is the result of collaboration between the Centers for Disease Control and Prevention and US states and territories since the 1980's. The BRFSS comprises large samples from the 50 federal states, as well as the District of Columbia. We chose the 2008 wave of the BRFSS for our analyses due to the temporal proximity to the EVS and removed the US territories Guam, Virgin Islands, and Puerto Rico from the data set.

For our analyses, we restricted the age range from 25 to 75 years to ensure that respondents' educational attainment process is completed and to remove health variation attributable to differential life expectancies across countries. Furthermore, we restricted the BRFSS to non-Hispanic Whites to explore variation beyond those attributable to racial health disparities in the US. This is a common practice in research comparing health disparities between the US and European countries (e.g. Avendano et al., 2009, 2011; Banks et al., 2006). The number of respondents in each European country is about $N=1,100$ and in each US state around $N=5,400$ (ranging from $N=646$ in Iceland to $N=16,567$ in Washington), which allows us to meaningfully compare differences between the federal states and European countries. Unfortunately, the comparatively small sample sizes in the EVS do not allow us to break down European countries into subnational units, for instance NUTS regions in the European Union. Exact sample sizes per country and federal state as well as name abbreviations are reported in Table A2.1.

Self-rated health Health status is ascertained from a single-item measure with five response options, although exact wording and response categories vary be- 
tween the surveys. The BRFSS measures self-rated health with ('Would you say that in general your health is ...') with five response categories 'Poor,' 'Fair,' 'Good,' 'Very good,' and 'Excellent.' The EVS asks 'All in all, how would you describe your state of health these days? Would you say it is ...', and the response options provided are 'Very poor,' 'Poor,' 'Fair,' 'Good,' and 'Very good.' Similar to Olafsdottir et al. (2013), we opted for a relative concordance interpretation (Jürges et al., 2008) to harmonize the response formats and count European respondents who reported 'Very poor' or 'Poor' health, as well as American respondents reporting 'Poor' or 'Fair' health as reporting 'Poor health.' This left us with poor health prevalence rates of 15.4 per cent and 10.3 per cent in the US and European samples, respectively.

Education Drawing on the ISCED-1997 classification (UNESCO, 2006), we distinguish between three key educational groups: Those not having completed high school (ISCED 0-2), those with a high school degree (ISCED 3), and those with further education (ISCED 4-6). There are several reasons for focusing on education for our purposes. Firstly, education reflects both individuals' material and non-material resources and their social status in a broad fashion. Secondly, the ISCED, with its high degree of cross-national standardization, allows to meaningfully compare educational groups across countries. Thirdly, educational attainment is usually completed in early adulthood and remains for the most part stable across the life course, thus reducing the chance of reverse causation: Unlike occupational status, educational degrees do not change even when an adult experiences a health shock (Elstad, 2004). Fourthly, compared to income, which usually comes with a large proportion of non-respondents, education is a comparably easy-to-measure indicator of socioeconomic status. Finally, education is a meaningful measure for the socioeconomic status of both men and women and for those outside of the labor force (Lahelma, 2001).

We use logistic regression models for each country and US state separately to model the log odds of reporting poor health, adjusted for sex and age. Firstly, based on these models, we predicted probabilities of reporting poor health for those with more than and less than high school. ${ }^{3}$ Secondly, we calculated the difference in adjusted prevalence rates of poor health in the two groups (less than minus

\footnotetext{
${ }^{3}$ We fit single-level logistic regression models for each country separately instead of relying on hierarchical random effects models for two reasons. Firstly, given the sample sizes in the US states and countries, the 'partial pooling' or 'borrowing strength' features of random effects models (Jones and Bullen, 1994) are not necessary for our purposes. Secondly, obtaining predicted probabilities from single-level nonlinear models strikes us more intuitive than obtaining them from a nonlinear model with a random effect (which will yield cluster-specific effects only, not population-averaged coefficients). But of course a logit random effects model would yield exactly the same predicted probabilities.
} 
Table 2.1: Descriptive statistics by sample

\begin{tabular}{llrr}
\hline Variable & Category & $\begin{array}{r}\text { US sample } \\
(N=269,105)\end{array}$ & $\begin{array}{r}\text { European sample } \\
(N=54,066)\end{array}$ \\
\hline Health & \% Poor & 15.4 & 10.3 \\
& \% Not poor & 84.6 & 89.8 \\
Education & \% Low & 5.6 & 28.5 \\
& \% Middle & 28.7 & 39.8 \\
& \% High & 65.7 & 31.7 \\
Sex & \% Male & 38.9 & \\
& \% Female & 61.1 & 54.5 \\
Age & $M$ & & \\
& $S D$ & 53.1 & 47.9 \\
& & 12.1 & 14.0 \\
\hline
\end{tabular}

more than high school) as a measure of absolute health inequalities. Finally, we calculated the ratio of the prevalence rates in the two groups (less than over more than high school), indicating relative health inequalities. 95 per cent confidence intervals (CI's) were obtained by means of the delta method.

\section{$2.3 \quad$ Results}

Results of our analyses are presented in Figure 2.1. The left panel of Figure 2.1 presents the overall prevalence rates of poor health in European countries and US states. There is a substantial variation, ranging from 3.0 per cent reporting poor health in The Netherlands to 27.8 per cent reporting poor health in West Virginia. European countries generally exhibit lower prevalence rates than the US, but this pattern has exceptions. The District of Columbia ranges among the European countries with a relatively low prevalence rate of poor health (5.5 per cent), and a number of Eastern European countries, such as Moldova, Georgia, Russia, and Ukraine, show prevalence rates in the range of the US states. In 43 US states, the prevalence rates of poor health were significantly above the European (EVS) average (dashed line).

The middle panel of Figure 2.1 shows the rate differences between the lower and higher educated respondents. In North Dakota, for instance, the rate difference is 11.6 per cent (95 per cent CI 8.2 to 15.1), indicating that the prevalence rate of poor health among the lower educated is 11.6 percentage points higher than among the higher educated. The fact that 0 is not included in the 95 per cent 

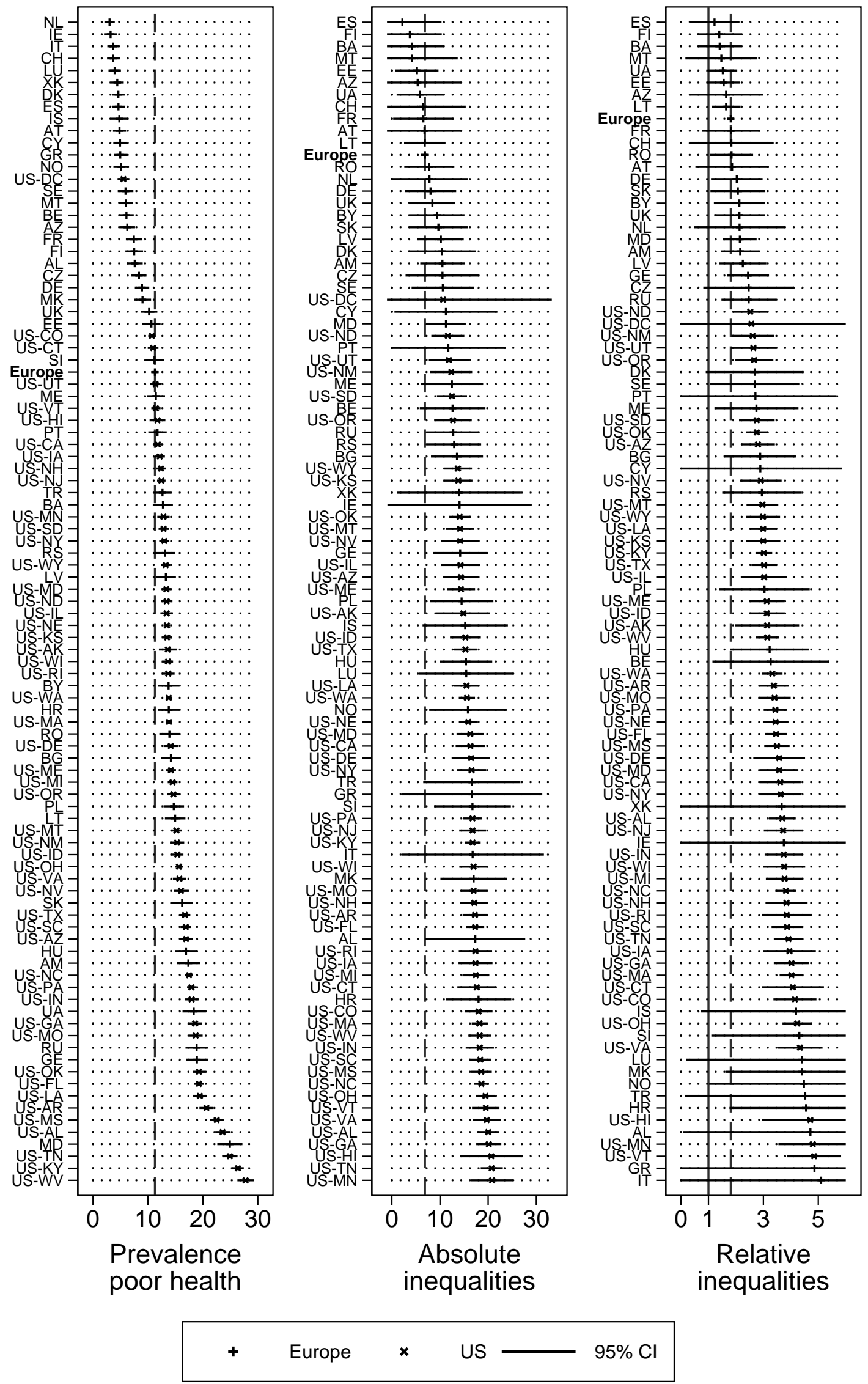

Figure 2.1: For caption see top of next page. 
Figure 2.1: Left panel: Prevalence rate and 95\% CI of reporting poor health in Europe and the US; middle panel: absolute inequalities in reporting poor health (rate difference low educated - high educated) and 95\% CI; right panel: relative inequalities in reporting poor health (rate ratio low educated over high educated). Everything adjusted for age and gender.

Notes: Dashed lines denote population-weighted EVS averages. 95\% CI's in middle panel truncated when $<-1,95 \% \mathrm{CI}$ 's in right panel truncated when $<0$ or $>6$.

confidence interval indicates that this difference reaches a level of conventional statistical significance. As was to be expected, poor health is generally more prevalent among the lower educated than among the higher educated. Comparing the absolute inequalities shows that differences are usually higher in the US, but a substantial number of European countries exhibit rate differences similar to those found in the US. Croatia (18.0 per cent), Albania (17.3 per cent), Italy (16.8 per cent), and Slovenia (16.7 per cent) are the most striking examples for large absolute health inequalities in Europe. All US states (except for Washington, DC) exhibited greater rate differences than the European average.

The right panel of Figure 2.1 shows the relative inequalities in poor health as expressed by the rate ratio of poor health among the lower educated compared to the higher educated. For example, in Lithuania, the rate ratio amounts to 1.6 (95 per cent CI 1.1 to 2.2), denoting that the lower educated are 60 per cent more likely to report poor health compared to the higher educated. In a number of countries and states, 95 per cent confidence intervals of the rate ratios include the value 1 , indicating that the point estimates are not statistically significant at the conventional level. Among those places where the rate ratio is significantly different from 1, the range is from 1.6 in Lithuania to 4.8 in Vermont. Again, while a large number of European countries exhibit substantially lower rate ratios than the US federal states, many European countries have ratios similar to those of the US, e.g. Croatia (4.6), Norway (4.5), or Macedonia (4.4). The dashed line indicates that all US states except the District of Columbia show greater relative inequalities than Europe.

\subsection{Discussion}

By and large, both prevalence of poor health and education-related inequality in poor health are greater in the US than in Europe. We reveal substantial within-US variation and show that differences between the US and Europe are not as clearcut as is often assumed. Some European countries resemble US states in terms of poor health prevalence and health inequalities. Although rankings differ when 
comparing absolute and relative inequalities, differences between Europe and the US are not straightforward.

While the striking resemblance between some Eastern European countries and large parts of the US might seem puzzling at first sight, research has pointed out some similarities between the health care systems of the US and those of Eastern Europe. The US suffer from a lack of universal health care access, and some problems of the health care system in Eastern Europe, like its weakness of primary care, its specialist orientation, and great fragmentation (Rechel et al., 2013) can also be found in the US. Furthermore, similarities in the often rudimentary conditions of social policies, both in the US and in Eastern Europe, could provide explanations for the patterns revealed in this study (Avendano and Kawachi, 2014; Fuchs and Offe, 2009; Hacker, 2006).

But as stated earlier, health care cannot be the main driver of the EuropeUS differences. Identifying upstream factors which can account for the differences between countries and states will be a major challenge for future research. Income inequality has been suggested to plausibly be a driver of health disparities (Beckfield et al., 2013; Wilkinson and Pickett, 2008). Despite the doubts about the causality between income inequality and population health (Beckfield, 2004; Lynch et al., 2004), there might be a link to health inequalities via complex pathways. Creating a comparable database for comprehensive institutional factors that might serve as explanations not only on the national level, but also on subnational levels, would be an important next step.

Also between European countries, the substantial heterogeneity in terms of health inequalities is remarkable. For some inequality indicators, countries such as Norway, known for their egalitarian policies, exhibit major health inequalities. Our findings corroborate existing research in this respect (Brennenstuhl et al., 2012), and the reasons for this variation are still being examined (Huijts and Eikemo, 2009; Huijts et al., 2010a; Mackenbach, 2012). Within the US, the country which we were able to break down into subnational units and where public welfare provision is generally low, variation in health inequalities was also substantial; therefore, egalitarian welfare policies might also not be the sole driver for health inequalities. Studies have demonstrated that also within European countries with largely centralized welfare policies, health inequalities vary across regions (e.g. Kibele, 2012; Kibele et al., 2014). Further comparative research into health inequalities should account for subnational levels that, as demonstrated in our study, exhibit a substantial heterogeneity of their own. While looking at heterogeneity within the US only and not within European countries is a shortcoming of our study, future research should identify datasets that allow for more detailed comparisons within Europe. Although the EVS is the largest contemporaneous European micro- 
dataset in terms of country coverage, the sample sizes within countries prohibit further breakdowns of the data to subnational levels. Future research should also focus on other health indicators, specifically ones that are more straightforward to compare across countries.

In conclusion, while some US states are on par with Western European countries regarding the size of the gradient, many have gradients that resemble those found in Eastern European countries. Our exploratory study cannot offer a comprehensive explanation of the variation in health inequalities we demonstrated. Given the recent advocacy for global comparisons of health inequalities (Beckfield et al., 2013; Olafsdottir et al., 2013), our study serves as a reminder to not overlook levels of variation within countries and that understanding local health conditions can give important cues for improving population health.

\section{Appendix}

Table A2.1: Country and US state codes and sample sizes

\begin{tabular}{|c|c|c|c|c|c|}
\hline Abb. & Country & $N$ & Abb. & Federal state & $N$ \\
\hline $\mathrm{AL}$ & Albania & 1,172 & US-AK & Alaska & 1,643 \\
\hline $\mathrm{AM}$ & Armenia & 1,151 & US-AL & Alabama & 3,756 \\
\hline AT & Austria & 1,223 & US-AR & Arkansas & 3,693 \\
\hline $\mathrm{AZ}$ & Azerbaijan & 1,137 & US-AZ & Arizona & 3,370 \\
\hline $\mathrm{BA}$ & Bosnia Herzegovina & 1,217 & US-CA & California & 5,950 \\
\hline $\mathrm{BE}$ & Belgium & 1,232 & US-CO & Colorado & 8,334 \\
\hline BG & Bulgaria & 1,241 & US-CT & Connecticut & 3,868 \\
\hline BY & Belarus & 1,155 & US-DC & District of Columbia & 1,697 \\
\hline $\mathrm{CH}$ & Switzerland & 1,058 & US-DE & Delaware & 2,699 \\
\hline $\mathrm{CY}$ & Cyprus & 1,159 & US-FL & Florida & 7,050 \\
\hline $\mathrm{CZ}$ & Czech Republic & 1,459 & US-GA & Georgia & 3,379 \\
\hline $\mathrm{DE}$ & Germany & 1,771 & US-HI & Hawaii & 1,900 \\
\hline DK & Denmark & 1,278 & US-IA & Iowa & 4,655 \\
\hline $\mathrm{EE}$ & Estonia & 1,232 & US-ID & Idaho & 3,942 \\
\hline ES & Spain & 1,166 & US-IL & Illinois & 3,318 \\
\hline FI & Finland & 1,000 & US-IN & Indiana & 3,330 \\
\hline FR & France & 1,224 & US-KS & Kansas & 6,296 \\
\hline GE & Georgia & 1,232 & US-KY & Kentucky & 6,442 \\
\hline GR & Greece & 1,209 & US-LA & Louisiana & 3,798 \\
\hline $\mathrm{HR}$ & Croatia & 1,130 & US-MA & Massachusetts & 13,388 \\
\hline
\end{tabular}


Table A2.1: Country and US state codes and sample sizes (cont'd)

\begin{tabular}{|c|c|c|c|c|c|}
\hline Abb. & Country & $N$ & Abb. & Federal state & $N$ \\
\hline $\mathrm{HU}$ & Hungary & 1,198 & US-MD & Maryland & 5,902 \\
\hline IE & Ireland & 781 & US-ME & Maine & 5,435 \\
\hline IS & Iceland & 646 & US-MI & Michigan & 5,963 \\
\hline IT & Italy & 1,203 & US-MN & Minnesota & 3,368 \\
\hline $\mathrm{LT}$ & Lithuania & 1,190 & US-MO & Missouri & 3,641 \\
\hline $\mathrm{LU}$ & Luxembourg & 1,115 & US-MS & Mississippi & 4,369 \\
\hline LV & Latvia & 1,184 & US-MT & Montana & 4,977 \\
\hline $\mathrm{MD}$ & Moldova & 1,188 & US-NC & North Carolina & 10,099 \\
\hline $\mathrm{ME}$ & Montenegro & 1,232 & US-ND & North Dakota & 3,885 \\
\hline MK & Macedonia & 1,242 & US-NE & Nebraska & 12,191 \\
\hline MT & Malta & 1,222 & US-NH & New Hampshire & 5,404 \\
\hline NL & Netherlands & 1,260 & US-NJ & New Jersey & 6,898 \\
\hline $\mathrm{NO}$ & Norway & 916 & US-NM & New Mexico & 2,920 \\
\hline PL & Poland & 1,183 & US-NV & Nevada & 3,014 \\
\hline $\mathrm{PT}$ & Portugal & 1,252 & US-NY & New York & 4,987 \\
\hline $\mathrm{RO}$ & Romania & 1,207 & US-OH & Ohio & 9,069 \\
\hline $\mathrm{RS}$ & Serbia & 1,241 & US-OK & Oklahoma & 4,930 \\
\hline RU & Russian Federation & 1,166 & US-OR & Oregon & 3,547 \\
\hline $\mathrm{SE}$ & Sweden & 1,046 & US-PA & Pennsylvania & 8,974 \\
\hline SI & Slovenia & 1,098 & US-RI & Rhode Island & 3,286 \\
\hline SK & Slovak Republic & 1,263 & US-SC & South Carolina & 5,529 \\
\hline $\mathrm{TR}$ & Turkey & 1,928 & US-SD & South Dakota & 4,884 \\
\hline $\mathrm{UA}$ & Ukraine & 1,222 & US-TN & Tennessee & 3,651 \\
\hline UK & United Kingdom & 1,527 & US-TX & Texas & 5,518 \\
\hline \multirow[t]{7}{*}{$\mathrm{XK}$} & Kosovo & 1,110 & US-UT & Utah & 4,075 \\
\hline & & & US-VA & Virginia & 3,444 \\
\hline & & & US-VT & Vermont & 5,458 \\
\hline & & & US-WA & Washington & 16,567 \\
\hline & & & US-WI & Wisconsin & 5,129 \\
\hline & & & US-WV & West Virginia & 3,322 \\
\hline & & & US-WY & Wyoming & 6,161 \\
\hline
\end{tabular}




\section{Chapter 3}

\section{Subjective Socioeconomic Status and Health in Cross-National Comparison $^{1}$}

\subsection{Introduction}

The relationship between objective and subjective socioeconomic status (SES) is a classic topic within sociology (Evans and Kelley, 2004; Lindemann and Saar, 2014; Marx, 1976; Wright, 1997), which has recently resurfaced in public health research (Adler, 2013; Nobles et al., 2013; Singh-Manoux et al., 2005; Wolff et al., 2010a). While sociological research on the issue long focused on class conflict and the potential for social revolution, public health research has discovered a robust association between subjective SES and a diverse range of health outcomes, usually over and above the influence of objective measures of social status. The general finding appears to be that those with a higher self-perception rating of their socioeconomic status enjoy better health (Adler, 2013).

Contrary to objective, long-established measures of socioeconomic status like education, income, and occupational prestige, subjective socioeconomic status is a self-appraisal about their location in a socioeconomic status order (Ross and Mirowsky, 2002). Terms that are sometimes used synonymously are perceived social position (Garbarski, 2010) and subjective social status (Adler et al., 2000; Demakakos et al., 2008).

The great recent interest in subjective SES among public health researchers

\footnotetext{
${ }^{1}$ This Chapter is co-authored with Melinda Mills and Rafael Wittek. Previous drafts of this Chapter were presented at the RC 28 Spring Meeting 2014 in Budapest, where it received the Aage B. Sørensen Award, and at the 2015 Annual Meeting of the Population Association of America in San Diego, CA. The authors thank discussants at these occasions for their comments as well as Marijtje van Duijn for help.
} 
has two reasons. Firstly, the subjective SES-health link has great potential to shed additional light onto the effects of social hierarchy on health. One strand of research, inspired by the works of Wilkinson (1992), suggests that subjective socioeconomic status reflects the relative rather than absolute position in the hierarchy of a society, and that the perception of inequality and subordination in the hierarchy of a society has damaging effects on health outcomes. Secondly, a more methodological reason for the relevance of the subjective SES-health relationship, is the interest in the general performance of subjective SES as a general marker of SES compared to other indicators like income or education. Some public health researchers, for instance, Singh-Manoux et al. (2005), suggest that subjective socioeconomic status could be a 'cognitive average' of objective SES markers, yielding a more precise measurement of overall SES.

With our study, we aim to shed light onto previously understudied aspects of the relationship between subjective SES and health, namely studying how this relationship operates in cross-national comparison. Our study extends existing research in three ways.

Firstly, while existing comparative research on health inequalities has so far focused on objective SES indicators, such as education (Eikemo et al., 2008a; Mackenbach et al., 2008), income (Huijts et al., 2010a), or class (Eikemo et al., 2008b), our study will extend that line of research by focusing on an innovative SES measure, namely subjective SES. Different indicators of SES cannot be used interchangeably (Torssander and Erikson, 2010), as they all tap at different, loosely related aspects of SES and vary in the strength of their association to health. Given the variation in levels of subjective SES across countries (Lindemann and Saar, 2014), we expect that comparing subjective SES-health gradients across societies is a valuable contribution to the literature.

Secondly, while previous research has established the health effects of subjective SES in a wide variety of population groups (e.g Adler et al., 2000; Quon and McGrath, 2014; Singh-Manoux et al., 2003), studies making use of representative population samples are scarce. Understanding the interplay of objective and subjective SES, however, requires samples that are free from selection bias, including all SES groups of a population, as associations found in restricted samples might misrepresent those apparent in the general population.

Thirdly, we present the first cross-national study on the subjective SES-health relationship. While not all existing studies have focused on US and UK samples (e.g. Karvonen and Rahkonen, 2011; Nobles et al., 2013), the vast majority has done so, and no existing study has compared differences in the strength of the subjective SES-health relationship across different societies. In fact, a recent review article on subjective SES and health explicitly demanded more cross-nationally 
comparative research on the subjective SES-health relationship (Euteneuer, 2014). Drawing on comparable data from 27 societies from all parts of the world, we will explore the variability in the relationship between subjective SES and health. By doing so, we will thus contribute to the recent 'comparative turn' in research on health inequalities (Beckfield et al., 2013; Eikemo et al., 2008a; Olafsdottir et al., 2013). Comparing the extent of health inequalities across countries allows to put them into context and gives an fresh impression of which inequalities can considered to be 'large' or 'small' (Olafsdottir et al., 2013). Furthermore, comparing health inequalities across societies allows identifying contextual factors, such as income inequality or welfare state policies, that can affect the size of socioeconomic gradients in health. In turn, comparative health inequalities research can give researchers and policy makers a sense of the malleability of health inequalities.

Exploiting the recently released ISSP 2011 data on "Health and Health Care," we will make use of multilevel modeling (random and fixed effects models) to explore variation in the subjective SES-health relationship across 27 countries, examining two distinct yet interrelated aspects of health, namely self-reported overall health, as well as psychological wellbeing.

\subsection{Theoretical background}

\subsubsection{The relationship between objective and subjective socioeconomic status (SES)}

The relationship between objective and subjective SES has long been a central concern of classical sociology. A traditional materialist argument has been the one of 'direct reflection' (Evans and Kelley, 2004): The objective working conditions and the relationship to the means of production lead the working classes to realize that they are at the bottom of the social hierarchy (Marx, 1975, 1976), which leads to an image of a polarized society with a sparsely populated elite at the top and a large working class at the bottom of society. Durkheim (1933) generally concurred with the notion that individuals' objective positions are accurately perceived by them, but maintained that the demand for skilled labor and greater occupational differentiation would give rise to a middle class, creating a more middle-heavy hierarchy in a society. It was Weber (1968) who highlighted the distinction between class and status, arguing that they are different but interrelated aspects of the stratification of a society, with class being primarily determined by relations on the labor market and status being a relational aspect of social honor that is reflected in patterns of association and lifestyles (Chan and Goldthorpe, 2007). 
Empirical research, often inspired by social psychology, has empirically backed up Weber's position, often finding objective conditions being a strong yet hardly all-determining predictor of subjective SES (Evans and Kelley, 2004; Jackman and Jackman, 1973). A proposed explanation is that perceptions of one's own social location are strongly shaped by one's social environment, such as family and friends. Given innate preferences of homophily, these form a biased sample, always leading individuals to believe they are located towards the middle of the social hierarchy (Evans and Kelley, 2004).

While classical sociology has thus raised the issue of tensions between objective and subjective SES, the consequences of the different aspects of SES have remained largely unexplored.

\subsubsection{The subjective SES-health relationship}

Public health research was able to amass substantial evidence for the existence of an association between subjective socioeconomic status and health. Health outcomes linked to subjective socioeconomic position included self-rated overall health (Demakakos et al., 2008; Singh-Manoux et al., 2005; Wolff et al., 2010b), depression (Demakakos et al., 2008; Sakurai et al., 2010; Singh-Manoux et al., 2003), nurserated health (Nobles et al., 2013), cortisol (Adler et al., 2000; Wright and Steptoe, 2005), obesity (Goodman et al., 2003), and mortality rates (Kopp et al., 2004). While some studies showed that the association between subjective socioeconomic status and health was explained when accounting for objective markers of SES, at least for some outcomes (Singh-Manoux et al., 2003), the majority of studies suggests that subjective SES is associated with health even after controlling for objective SES.

These findings do not only pertain to US or UK samples (Demakakos et al., 2008; Garbarski, 2010; Operario et al., 2004; Seeman et al., 2014; Singh-Manoux et al., 2003, 2005), a number of studies also drew on samples from other regions, such as Finland (Karvonen and Rahkonen, 2011), Hungary (Kopp et al., 2004), Indonesia (Nobles et al., 2013), Japan (Sakurai et al., 2010), Taiwan (Collins and Goldman, 2008), or Canada (Dunn et al., 2006). While many of the studies focused on select populations, such as pregnant women (Ostrove et al., 2000; Reitzel et al., 2007), adolescents (Goodman et al., 2001, 2003; Karvonen and Rahkonen, 2011; Quon and McGrath, 2014), older adults (Collins and Goldman, 2008; Demakakos et al., 2008; Garbarski, 2010), participants of an experiment (Cohen et al., 2008), or civil service workers (Singh-Manoux et al., 2003, 2005), relatively few used representative samples of the general population (Nobles et al., 2013; Sakurai et al., 2010; Wolff et al., 2010b). 
How can the relationship between subjective SES and health be interpreted? Research has so far pointed out two interpretations. Firstly, the subjective measure picks up aspects of SES that remain unmeasured by objective markers of SES. Subjective SES has been suggested to give a more nuanced reflection of an individual's socioeconomic standing. On the one hand, taking into account socioeconomic characteristics that are relatively easy to observe, such as income or educational degrees attained (Singh-Manoux et al., 2005). On the other hand, subjective socioeconomic status also reflects more difficult to gauge aspects of socioeconomic status, such as past and future prospects. This explanation is in line with the apparent popularity of using subjective measures to assess the SES of adolescents (Quon and McGrath, 2014), given the difficulties of accurately assessing SES of adolescents who usually have not yet finished their SES attainment process. Secondly, the association of health with the subjective measure reflects the harm to health caused by the cognitive and emotional reactions to lower status positions. Experiencing lower status has been suggested to have negative health consequences in itself (Layte and Whelan, 2014; Marmot, 2004; Wilkinson and Pickett, 2010), operating via stress-related neuroendocrinological pathways (Dickerson and Kemeny, 2004) and poor health behaviors such as smoking or overeating (Layte and Whelan, 2009). These interpretations are not necessarily mutually exclusive, they can both operate at the same time.

An important function of cross-national research is to confirm the presence of relationships found in single-context studies in a variety of contexts. Based on the mass of research findings, we pose the following hypotheses:

H1A: Subjective SES is positively related to health in all countries under study.

H1B: Subjective SES is positively related to health in all countries under study after accounting for objective measures of SES (household income, education, and occupational prestige).

\subsubsection{Country affluence, income inequality, and the sub- jective SES-health relationship}

Two major contextual factors that are frequently discussed in the literature on social determinants of health are the economic resources of a country, most commonly expressed as GDP per capita, and income inequality, usually expressed as the Gini coefficient. While most of the current literature focuses on the direct effects of country affluence and income inequality on health, we will extend this literature by making a case that both these factors can have moderating effects on 
the subjective SES-health relationship.

The effects of country affluence on population health have been variously and prominently demonstrated (Deaton, 2013). Populations flourish in terms of health when economic resources are available in great quantity. Societies with greater resources available in the infrastructure can benefit all their members, reducing the importance of individual perceptions for health and wellbeing. In line with the notion of 'A rising tide lifts all boats,' greater wealth in a country might decrease the strength of the subjective SES-health relationship. Semyonov et al. (2013) also suggest that the availability of resources in a country could reduce the relationship between SES and health, as individual command over resources becomes less important. The same could be true for the subjective SES-health relationship, as status competition might be less crucial as long as basic needs are met.

H2: The subjective SES-health association is weaker in countries with greater affluence.

Some researchers, however, have pointed out that the relationship between country affluence becomes unimportant for population health as soon as a certain threshold of wealth has been surpassed (Wilkinson, 1997; Wilkinson and Pickett, 2010). After that level has been reached, it is presumably income inequality that becomes the important driver of population health (Wilkinson and Pickett, 2010). The debate about the relationship between income inequality and health has been elaborately discussed in the literature (Beckfield, 2004; Ellwardt et al., 2014; Kondo et al., 2009; Layte and Whelan, 2014; Lynch et al., 2004; Präg et al., 2014), however, here we would like to focus on any moderating effects of income inequality on the subjective SES-health association.

A few previous studies have suggested that income inequality might exacerbate health inequalities (Beckfield et al., 2013; Semyonov et al., 2013; Wilkinson and Pickett, 2008). Beckfield et al. (2013) suggest a 'fundamental cause' (Phelan et al., 2010) explanation for this hypothesized relationship. High-SES invididuals in less egalitarian societies might have even more resources that they can translate more easily into better health, leaving the disadvantaged even further behind in terms of health. Also, given that income can serve as a buffer for the stress individuals face in their lives, low-income individuals in less egalitarian societies should be more stressed and, thus, less healthy, exacerbating the health gradient in less egalitarian countries. Semyonov et al. (2013) point to the neo-materialist pathway (Lynch et al., 2000) that is suggested to connect income inequality and average population health. According to this pathway, societies with a high degree of income inequality are also characterized by a country's systematic underinvestment 
across a wide range of human, physical, and social infrastructures. The less well-off are likely to suffer most from these underinvestments, as they lack the personal resources to make up for these public underinvestments, thus, it is reasonable to expect that health inequalities in countries with greater income inequality should be greater as well. Wilkinson and Pickett (2008) suggest that status competition should be stronger in places characterized by greater income inequality, which exacerbates health inequalities via a status differentiation pathway.

The empirical evidence, however, has been mixed. Semyonov et al. (2013) report that any exacerbating effect of income inequality on the relationship between household wealth and health in their sample of countries is solely driven by the US. For the other, mostly Western European countries in their sample, they do not find any relationship between income inequality and health. Beckfield et al. (2013) study a heterogeneous sample of countries from around the world and find a weak moderating effect of income inequality on the association between income and selfrated health, but a sizable interaction between income inequality, education, and self-rated health in a country; the more unequal a society in terms of income, the stronger the relationship between education and self-rated health. Wilkinson and Pickett (2008) study counties in the US and are able to show that mortality rates for different causes of death, which are more strongly related to median county income, are also more strongly correlated with income inequality, suggesting that there is indeed a link between income inequality and health gradients.

However, in the context of a subjective SES measure, one could expect that an interaction effect of income inequality and subjective SES would be strong evidence for the interpretation of subjective SES being a marker of the negative health effects of low social status. Wilkinson and Pickett (2010) argue that greater social inequality in a country makes status comparisons more painful, creating greater stress and leading to worse health outcomes for those lower in the social hierarchy. While evidence for this mechanism is so far mixed (Layte, 2012; Präg et al., 2014), the test proposed here tackles the issue from a new angle.

H3: The subjective SES-health association is stronger in countries with greater income inequality.

\subsection{Data and method}

\subsubsection{Data}

Individual-level data Our analyses make use of the recently released 2011 International Social Survey Program (ISSP) module "Health and Health Care" 
(ISSP Research Group, 2013). Our analysis contains information from respondents from 27 countries from all major regions of the world, namely Australia (AU), Belgium (BE), Bulgaria (BG), Switzerland (CH), Chile (CL), the Czech Republic (CZ), Germany (DE), Denmark (DK), Finland (FI), France (FR), Croatia (HR), Israel (IL), Japan (JP), South Korea (KR), Latvia (LV), the Netherlands (NL), Norway (NO), the Philippines (PH), Poland (PL), Portugal (PT), Russia (RU), Sweden (SE), Slovenia (SI), Slovakia (SK), Turkey (TR), Taiwan (TW), and South Africa (ZA). The United Kingdom and the United States had to be excluded from our analyses, as the focal independent variable, subjective socioeconomic status, was not included in the data set. Realized sample sizes range from about 1,000 to 3,300 respondents per country. Interviews were conducted in the period 2011-2013 and response rates range between 30.2 per cent (Wallonian region of Belgium) and 85.9 per cent (South Africa).

We restrict the age range of respondents to $25-74$ years. The reasons for this is that on the one hand, we want to ensure most respondents have completed education, and on the other, we have to account for the fact that some countries used upper and lower age cut-offs during data collection. Sample sizes per country are reported in Appendix Table A3.1.

Country-level information We obtained information on income inequality from the Standardized World Income Inequality Database (SWIID, Solt, 2009) as expressed in Gini coefficients. A Gini of 0 indicates that all households in a country have exactly the same income (low inequality), whereas a Gini of 100 indicates high inequality (one household receives all income in a country, while no other households receive no income at all). Country affluence (GDP per capita, log transformed) information was obtained from the World Bank data base (World Bank, 2014b) and information for Taiwan was obtained from the International Monetary Fund (IMF, 2012). Descriptive statistics of country-level covariates are reported in Appendix Table A3.1.

\section{Outcome variables}

Self-rated overall health was measured with a single item: "In general, would you say your health is ... excellent (4), very good (3), good (2), fair (1), or poor (0)?" Self-rated health is a general assessment of one's health status, not connected to any specific illness, but covering largely physical and functional aspects of health (Idler et al., 1999). It has been shown to predict mortality and morbidity and has high test-retest reliability in a number of studies (Idler and Benyamini, 1997). Furthermore, this variable has been recommended by the WHO for comparative 
research (De Bruin et al., 1996), and a large number of researchers have followed this advice (e.g. Hildebrand and Van Kerm, 2009; Huijts and Kraaykamp, 2011), especially in the comparative study of health disparities (Beckfield et al., 2013; Mackenbach et al., 2008; Präg et al., 2014). Research has also shown that different socioeconomic groups evaluate their health in comparable ways (Burström and Fredlund, 2001) and that the associations between objective health indicators and self-perceived health are largely similar across countries (Bardage et al., 2005). In terms of clustering across countries, we find an intraclass correlation coefficient $(I C C)$ of .09. The $I C C$ gives the proportion of the total variance in a dependent variable that is accounted for by the clustering in countries. Put differently, the $I C C$ is a measure of the extent to which respondents living in the same country are more similar to one another than to respondents living in other countries.

As a second outcome variable, we are using a composite measure of psychological wellbeing based on three items on psychological distress reported in the last four weeks. Respondents were asked how often in the past four weeks they 'felt unhappy and depressed,' 'lost confidence in yourself,' and 'felt you could not overcome your problems.' Response options were 'never,' (0) 'seldom,' (1) 'sometimes,' (2) 'often,' (3) and 'very often' (4). Psychological wellbeing is a frequently used outcome variable in cross-national studies (e.g. Djundeva et al., 2015). A principal component analysis of the three items yields a clear, one-dimensional solution (explained variance 89 per cent); all three items exhibit factor loadings exceeding .88. Cronbach's alpha for the five items is .87 (range $\alpha=.72(\mathrm{PH})$ to $\alpha=.91$ $(\mathrm{LT})$ ), indicating high internal consistency in all countries under study. We calculated the average score of the three items, yielding a variable ranging from 0 to 4 , with higher values indicating greater psychological wellbeing. For psychological wellbeing, $I C C=.04$. A potential problem is that about 75 per cent of French respondents did not answer the questions about psychological wellbeing. For this reason, we exclude the entire French sample from the analyses of psychological wellbeing.

Both outcome variables correlate with $r=.39$ at the individual level, indicating that they that capture related, yet distinct aspects of health.

\section{Predictor variables}

The focal predictor variable of our study is subjective socioeconomic status. It was measured with the question: "In our society, there are groups which tend to be towards the top and groups which tend to be towards the bottom. Below is a scale that runs from the top to the bottom. Where would you put yourself on this scale?" Along with this question, respondents were presented a ladder, and rungs 
were assigned numbers from 1 to 10 , with 1 indicating the very bottom and 10 the very top rung of the ladder. This measure resembles the one introduced by Adler et al. (2000), which is frequently used in current research (Lindemann and Saar, 2014; Nobles et al., 2013). Cundiff et al. (2013) demonstrated the construct validity of the scale. Evans and Kelley (2004) make a case for the cross-national comparability of the question, pointing to 1) the simple, abstract structure of the question, facilitating comparability across countries; 2) the problems that would arise if respondents have to force themselves into a restricted, pre-assigned classscheme; and 3) its avoidance of in many countries politically charged terms like 'middle class' or 'working class.'

In order to assess objective socioeconomic status, we rely on three indicators. Education was classified according to the ISCED 1997 typology (UNESCO, 2006), distinguishing between the lower educated (ISCED 0-2), those with medium education (ISCED 3-4), and those with tertiary degrees (ISCED 5-6). Household income before taxes was equivalized by dividing it by the square root of the number of household members and transformed into country-specific income quintiles. For those respondents who failed to report their income, we added an additional category to retain them for our analyses (Cohen et al., 2003). Occupational prestige was assessed by creating ISEI scores (International Socio-Economic Index, Ganzeboom et al., 1992) based on the ISCO-88 occupational classification. Originally, the ISEI ranges from 16 to 90; to facilitate interpretation, we have rescaled the predictor by dividing it by 10 . For respondents who have never worked (and thus do not have an ISEI score), we included a dummy variable and imputed the average ISEI. By doing so, we can interpret the coefficient of the dummy indicator as the average difference between those who have never worked and those who have or had a job with an average ISEI (Allison, 2002).

We further control for age (measured in years), sex $(1=$ female, $0=$ male), and a set of dummies to control for legal marital status ('married/civil partnership' (ref.), 'separated/divorced,' 'widowed,' 'single/never married').

\subsubsection{Method and modeling strategy}

In order to test our hypotheses, we rely on multilevel (random coefficient) modeling (Snijders and Boskers, 2012; Subramanian et al., 2003). This allows us to account for the fact that the respondents in our sample are nested in countries. Furthermore, we can explicitly model between-country variation, while simultaneously accounting for compositional differences between countries. Our models include random intercepts, thus allowing for country-specific constant terms in the regression equations. To facilitate interpretation of interactions and the random 


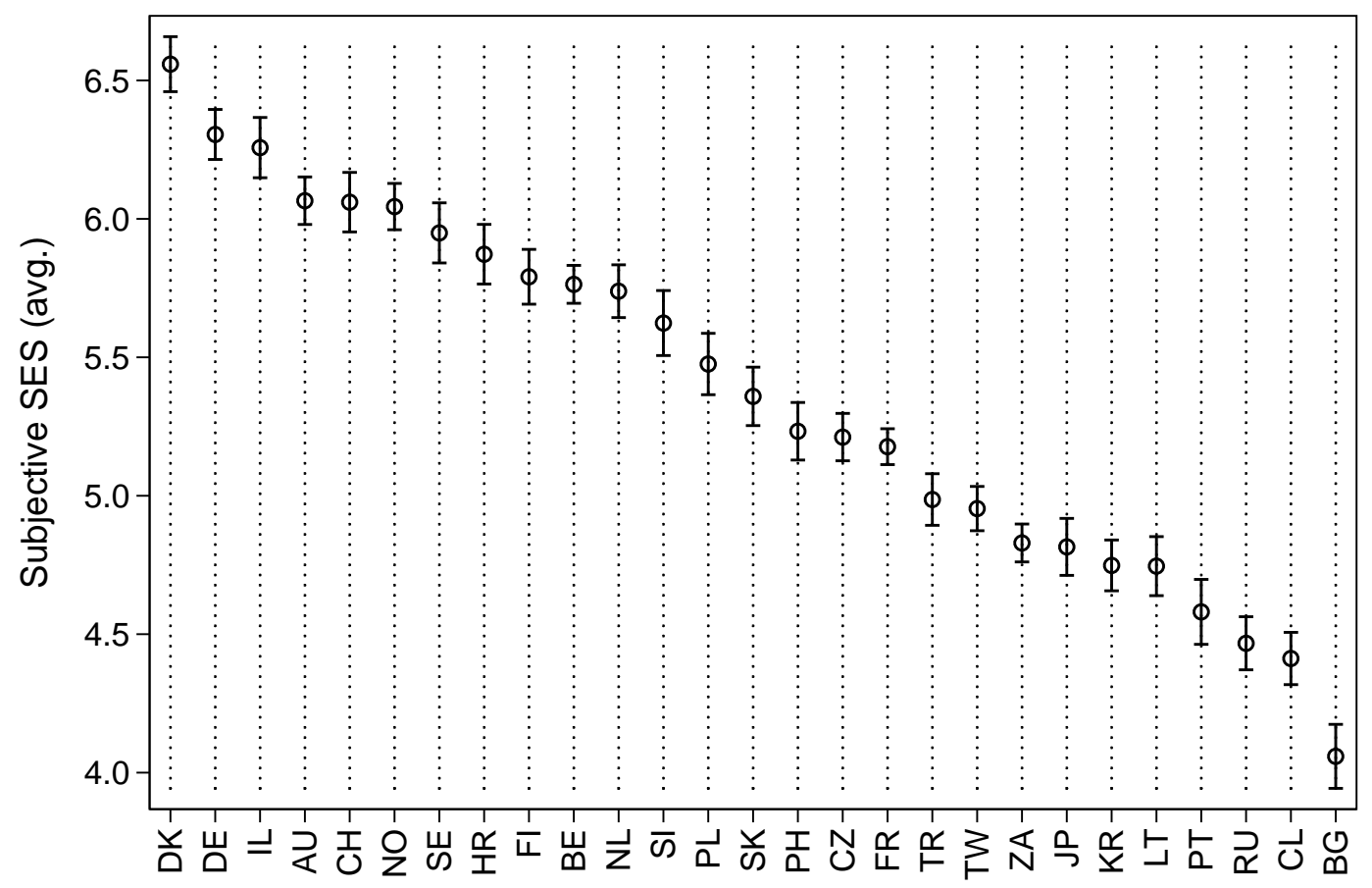

Figure 3.1: Average subjective SES by country Note: Error bars denote $95 \%$ CI's

components, all continuous predictor variables have been grand-mean centered. Given the focal interest in the cross-national variation of the subjective SEShealth relationship while only having a limited sample of countries, we replicated all analyses using two-step OLS regression models as a robustness check (Bryan and Jenkins, 2015; Kedar and Shively, 2005).

\subsection{Results}

\subsubsection{Descriptive findings}

Figure 3.1 displays the country averages in subjective SES across countries. Some interesting patterns emerge. By and large the country averages seem to follow national income, with individuals in high-GDP countries reporting higher subjective SES. However, this pattern is not without exceptions. For instance, Israelis report the third-highest subjective SES, right after the Danes and the Germans. The bottom of the ranking is occupied by Russia, Chile, and Bulgaria: three countries with a comparably low GDP. However, Portugal and Japan occupy the next-highest positions in the ranking.

Figure 3.2 presents descriptive findings on the country-level for the two health outcomes and subjective SES. As already reported for the micro-level, the two 


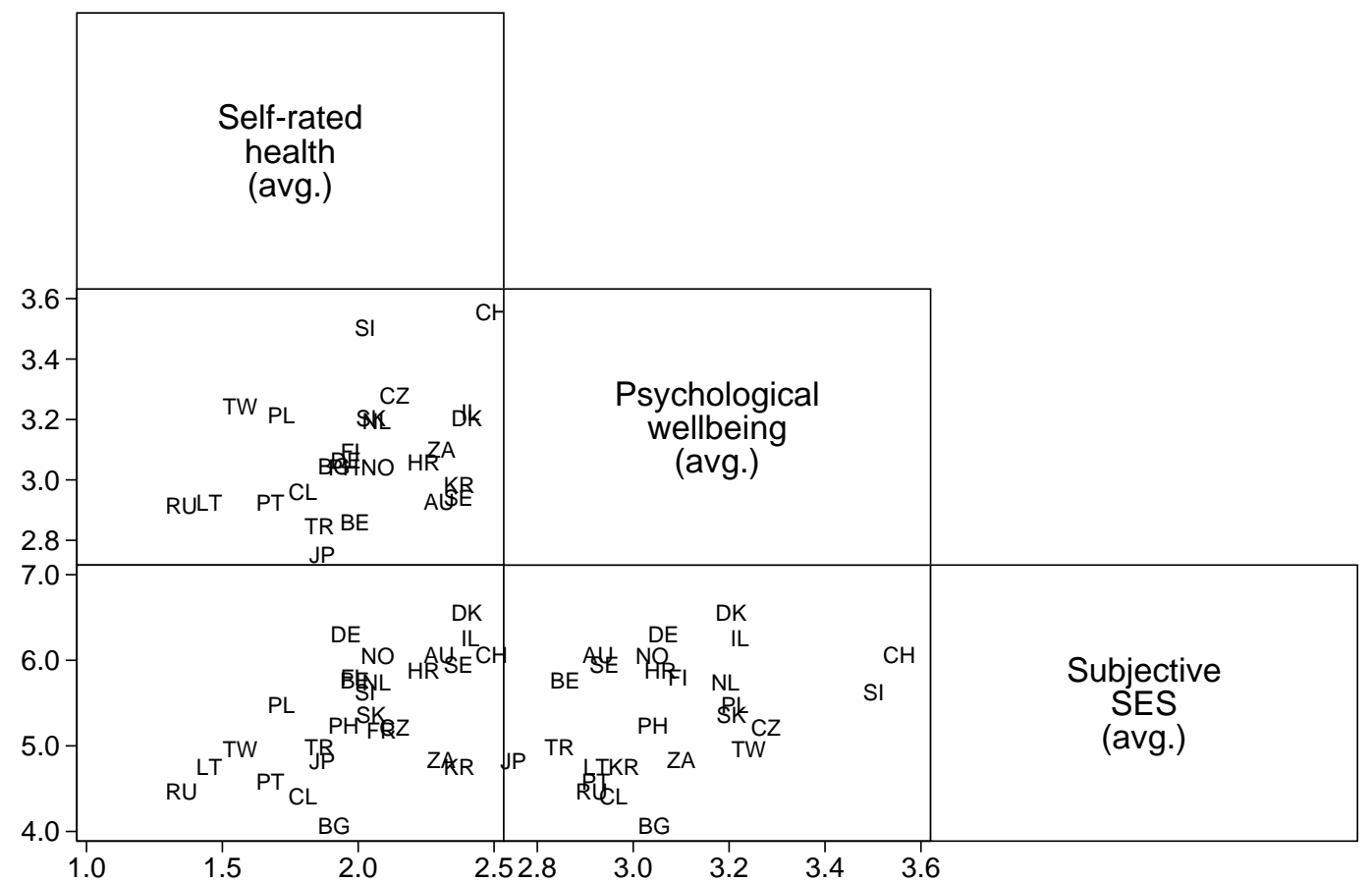

Figure 3.2: Scatterplot matrix of self-rated health, psychological wellbeing, and subjective SES on the country level

health outcomes are related to one another, however, the relationship appears to be rather modest (Pearson's $r=.34$ for 26 countries). We can also see that subjective SES aggregated to the country level predicts the two outcomes, but to varying degrees: While the relationship to self-rated overall health is sizable $(r=.60)$, the relationship to average psychological wellbeing in a country is more modest $(r=.34)$. Table 3.1 reports means and standard deviations from the individual-level variables under study. 
Table 3.1: Descriptive statistics of individual-level variables, $N=29,799$

\begin{tabular}{|c|c|c|c|c|}
\hline Variable & Mean & Std. dev. & Min. & $\operatorname{Max}$ \\
\hline Self-rated general health & 2.04 & 0.99 & 0 & 4 \\
\hline Psychological wellbeing & 3.09 & 0.93 & 0 & 4 \\
\hline Subjective SES (centered) & 0.00 & 1.80 & -4.40 & 4.60 \\
\hline \multicolumn{5}{|l|}{ Education } \\
\hline Low Education & 0.34 & - & 0 & 1 \\
\hline Medium Education & 0.39 & - & 0 & 1 \\
\hline High Education & 0.27 & - & 0 & 1 \\
\hline \multicolumn{5}{|l|}{ ISEI } \\
\hline ISEI (centered) & 0.00 & 16.0 & -27.3 & 46.7 \\
\hline Never worked & 0.08 & - & 0 & 1 \\
\hline \multicolumn{5}{|l|}{ Household income quintiles } \\
\hline Q 1 (lowest) & 0.15 & - & 0 & 1 \\
\hline Q 2 & 0.16 & - & 0 & 1 \\
\hline Q 3 & 0.16 & - & 0 & 1 \\
\hline Q 4 & 0.16 & - & 0 & 1 \\
\hline Q 5 (highest) & 0.17 & - & 0 & 1 \\
\hline Income missing & 0.20 & - & 0 & 1 \\
\hline Age (centered) & 0.00 & 13.6 & -23.6 & 25.4 \\
\hline Female & 0.56 & - & 0 & 1 \\
\hline \multicolumn{5}{|l|}{ Marital status } \\
\hline Married/cohabiting & 0.65 & - & 0 & 1 \\
\hline Divorced & 0.10 & - & 0 & 1 \\
\hline Widowed & 0.07 & - & 0 & 1 \\
\hline Single & 0.18 & - & 0 & 1 \\
\hline
\end{tabular}

\subsubsection{Multivariate analyses}

Table 3.2 presents coefficients as obtained from random intercept models. In Models $1 \mathrm{a}$ and $1 \mathrm{~b}$, we find a small yet statistically significant relationship between subjective SES and health. For self-rated general health in Model 1a, the association with subjective SES amounts to 0.13. For each additional rung on the subjective SES ladder, self-rated health increases by 0.13 points. Given the fact that the standard deviation of self-rated health is close to 1.0, this also amounts 
to an increase of 0.13 standard deviations, which is a small yet statistically significant effect size. In Model 1b, which has psychological wellbeing as the outcome, findings are strikingly similar, with the subjective SES coefficient also being about 0.13. When standardizing this with the standard deviation of the psychological wellbeing variable ( $S D=0.93$ ), it shows that an additional rung on the subjective SES ladder goes along with an increase of 0.14 standard deviations in psychological wellbeing, which is again a small, but statistically significant effect size.

In both models, the control variables behave as expected. Age has a negative association with overall health and psychological wellbeing, women reported slightly worse health and wellbeing than men, and married/cohabiting individuals have better overall health and wellbeing than singles, the divorced, and especially the widowed. 
Table 3.2: Self-rated health and psychological wellbeing regressed on several predictors (random coefficient models)

\begin{tabular}{|c|c|c|c|c|c|c|}
\hline & $\begin{array}{c}\text { (1a) } \\
\text { Self-rated } \\
\text { general health }\end{array}$ & $\begin{array}{c}\text { (1b) } \\
\text { Psychological } \\
\text { wellbeing }\end{array}$ & $\begin{array}{c}(2 \mathrm{a}) \\
\text { Self-rated } \\
\text { general health }\end{array}$ & $\begin{array}{c}(2 \mathrm{~b}) \\
\text { Psychological } \\
\text { wellbeing }\end{array}$ & $\begin{array}{c}(3 \mathrm{a}) \\
\text { Self-rated } \\
\text { general health }\end{array}$ & $\begin{array}{c}\text { (3b) } \\
\text { Psychological } \\
\text { wellbeing }\end{array}$ \\
\hline Subjective SES & $\begin{array}{c}0.130^{* * *} \\
(42.47)\end{array}$ & $\begin{array}{c}0.125^{* * *} \\
(39.06)\end{array}$ & $\begin{array}{c}0.132^{* * *} \\
(20.52)\end{array}$ & $\begin{array}{c}0.124^{* * *} \\
(16.51)\end{array}$ & $\begin{array}{c}0.108^{* * *} \\
(16.69)\end{array}$ & $\begin{array}{c}0.109^{* * *} \\
(14.77)\end{array}$ \\
\hline \multicolumn{7}{|l|}{ Education (ref. low education) } \\
\hline Medium education & & & & & $\begin{array}{c}0.0883^{* * *} \\
(6.62)\end{array}$ & $\begin{array}{c}0.0373^{* *} \\
(2.64)\end{array}$ \\
\hline High education & & & & & $\begin{array}{c}0.121^{* * *} \\
(6.90)\end{array}$ & $\begin{array}{c}0.00258 \\
(0.14)\end{array}$ \\
\hline ISEI & & & & & $\begin{array}{c}0.00202^{* * *} \\
\quad(5.15)\end{array}$ & $\begin{array}{c}0.00119^{* *} \\
(2.89)\end{array}$ \\
\hline Never worked (ref. works/v & vorked) & & & & $\begin{array}{c}-0.00646 \\
(-0.29)\end{array}$ & $\begin{array}{c}-0.0564^{*} \\
(-2.48)\end{array}$ \\
\hline \multicolumn{7}{|l|}{ Income (ref. lowest quintile) } \\
\hline Second quintile & & & & & $\begin{array}{c}0.0817^{* * *} \\
\quad(4.47)\end{array}$ & $\begin{array}{c}0.117^{* * *} \\
(6.13)\end{array}$ \\
\hline Third quintile & & & & & $\begin{array}{c}0.0842^{* * *} \\
(4.56)\end{array}$ & $\begin{array}{c}0.189^{* * *} \\
(9.77)\end{array}$ \\
\hline Fourth quintile & & & & & $\begin{array}{c}0.141^{* * *} \\
(7.45)\end{array}$ & $\begin{array}{c}0.207^{* * *} \\
(10.40)\end{array}$ \\
\hline Fifth quintile & & & & & $\begin{array}{c}0.191^{* * *} \\
(9.72)\end{array}$ & $\begin{array}{c}0.210^{* * *} \\
(10.16)\end{array}$ \\
\hline Income missing & & & & & $\begin{array}{c}0.134^{* * *} \\
(7.38)\end{array}$ & $\begin{array}{c}0.194^{* * *} \\
(10.16)\end{array}$ \\
\hline Age & $\begin{array}{c}-0.0170^{* * *} \\
(-39.81)\end{array}$ & $\begin{array}{c}-0.00170^{* * *} \\
(-3.79)\end{array}$ & $\begin{array}{c}-0.0169^{* * *} \\
(-39.63)\end{array}$ & $\begin{array}{c}-0.00169^{* * *} \\
(-3.76)\end{array}$ & $\begin{array}{c}-0.0158^{* * *} \\
(-36.27)\end{array}$ & $\begin{array}{c}-0.00118^{*} \\
(-2.57)\end{array}$ \\
\hline Female (ref. male) & $\begin{array}{c}-0.0474^{* * *} \\
(-4.58)\end{array}$ & $\begin{array}{c}-0.171^{* * *} \\
(-15.77)\end{array}$ & $\begin{array}{c}-0.0475^{* * *} \\
(-4.60)\end{array}$ & $\begin{array}{c}-0.171^{* * *} \\
(-15.77)\end{array}$ & $\begin{array}{c}-0.0413^{* * *} \\
(-3.96)\end{array}$ & $\begin{array}{c}-0.161^{* * *} \\
(-14.65)\end{array}$ \\
\hline \multicolumn{7}{|c|}{ Marital status (ref. married/cohabiting) } \\
\hline Divorced & $\begin{array}{c}-0.0528^{* *} \\
(-3.09)\end{array}$ & $\begin{array}{c}-0.195^{* * *} \\
(-10.72)\end{array}$ & $\begin{array}{c}-0.0514^{* *} \\
(-3.00)\end{array}$ & $\begin{array}{c}-0.193^{* * *} \\
(-10.62)\end{array}$ & $\begin{array}{c}-0.0421^{*} \\
(-2.46)\end{array}$ & $\begin{array}{c}-0.178^{* * *} \\
(-9.77)\end{array}$ \\
\hline Widowed & $\begin{array}{c}-0.116^{* * *} \\
(-5.40)\end{array}$ & $\begin{array}{c}-0.210^{* * *} \\
(-9.34)\end{array}$ & $\begin{array}{c}-0.116^{* * *} \\
(-5.39)\end{array}$ & $\begin{array}{c}-0.207^{* * *} \\
(-9.20)\end{array}$ & $\begin{array}{c}-0.0924^{* * *} \\
(-4.30)\end{array}$ & $\begin{array}{c}-0.179^{* * *} \\
(-7.98)\end{array}$ \\
\hline Single & $\begin{array}{c}-0.0427^{* *} \\
(-2.93)\end{array}$ & $\begin{array}{c}-0.129^{* * *} \\
(-8.38)\end{array}$ & $\begin{array}{c}-0.0417^{* *} \\
(-2.87)\end{array}$ & $\begin{array}{c}-0.124^{* * *} \\
(-8.04)\end{array}$ & $\begin{array}{c}-0.0427^{* *} \\
(-2.94)\end{array}$ & $\begin{array}{c}-0.116^{* * *} \\
(-7.56)\end{array}$ \\
\hline Intercept & $\begin{array}{c}2.072^{* * *} \\
(42.31)\end{array}$ & $\begin{array}{c}3.242^{* * *} \\
(98.50)\end{array}$ & $\begin{array}{c}2.065^{* * *} \\
(42.67)\end{array}$ & $\begin{array}{c}3.242^{* * *} \\
(94.70)\end{array}$ & $\begin{array}{c}1.884^{* * *} \\
(36.91)\end{array}$ & $\begin{array}{c}3.064^{* * *} \\
(83.32)\end{array}$ \\
\hline Variance (intercept) & $0.0628^{* * *}$ & $0.0261^{* * *}$ & $0.0612^{* * *}$ & $0.0283^{* * *}$ & $0.0620^{* * *}$ & $0.0262^{* * *}$ \\
\hline Variance (residual) & $0.758^{* * *}$ & $0.762^{* * *}$ & $0.756^{* * *}$ & $0.759^{* * *}$ & $0.747^{* * *}$ & $0.753^{* * *}$ \\
\hline Variance (subjective SES) & & & $0.000836^{* * *}$ & $0.00117^{* * *}$ & $0.000809^{* * *}$ & $0.00107^{* * *}$ \\
\hline Covariance (subjective SES & , intercept) & & 0.001 & -0.002 & 0.00117 & -0.00246 \\
\hline Deviance & 76431.8 & 69879.8 & 76384.4 & 69807.3 & 76045.2 & 69589.4 \\
\hline$N$ countries & 27 & 26 & 27 & 26 & 27 & 26 \\
\hline$N$ & 29,799 & 27,191 & 29,799 & 27,191 & 29,799 & 27,191 \\
\hline
\end{tabular}




\section{Empirical Bayes regression lines}
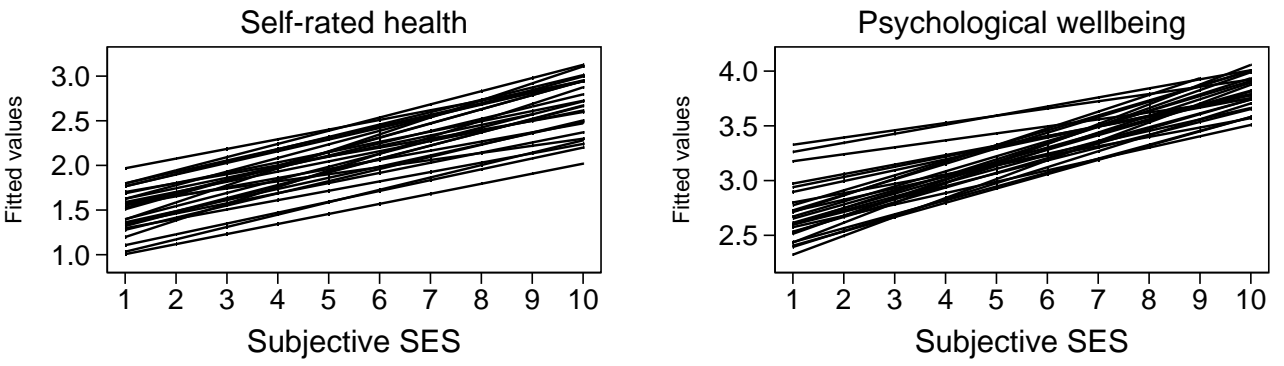

\section{Empirical Bayes residuals \\ not accounting for objective SES indicators}
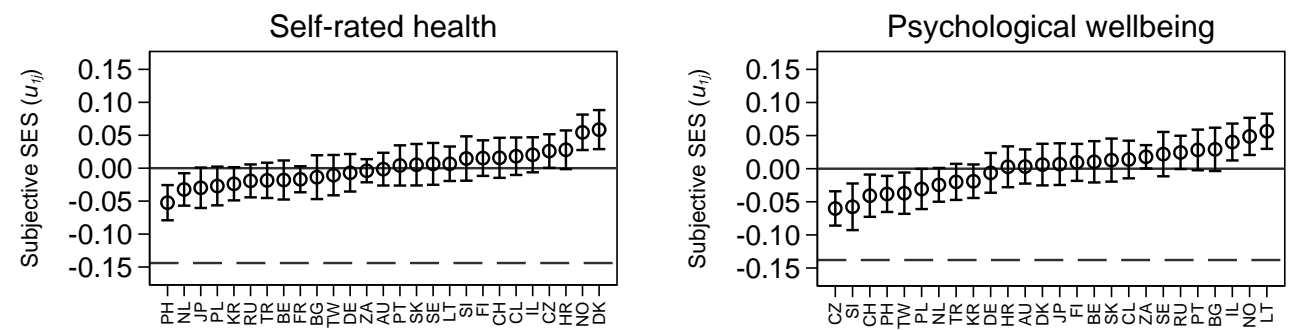

\section{Empirical Bayes residuals accounting for objective SES indicators}
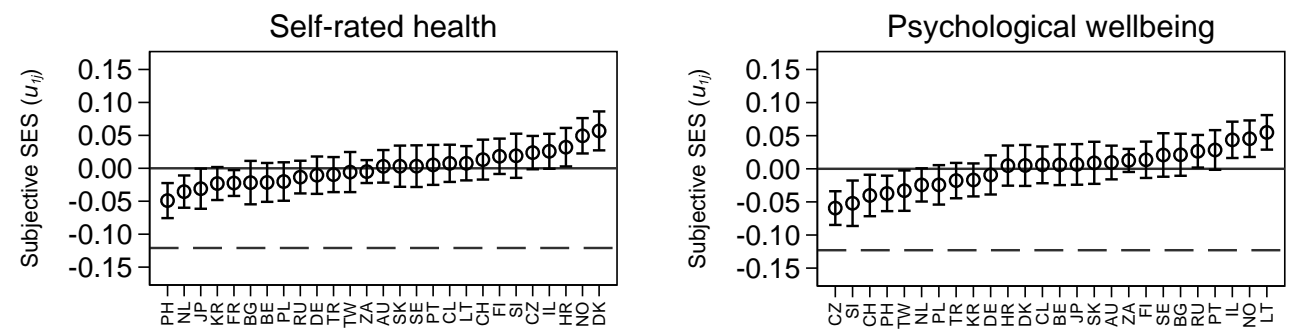

Figure 3.3: Panels in top row: Empirical Bayes regression lines based on Models 2a (left panel) and 2b (right panel) for all countries. Middle row: Caterpillar plots of the association between subjective SES and health outcomes across countries based on Models 2a (left panel) and 2b (right panel). Bottom row: Caterpillar plots of the association between subjective SES and health outcomes across countries based on Models 3a (left panel) and 3b (right panel)

Notes: Dashed lines in middle and lower panels denote the upper bounds of the $95 \%$ CI's of the fixed coefficient of subjective SES, as obtained from Models $2 \mathrm{a}$ and $2 \mathrm{~b}$ (middle row) and

Models 3a and 3b (lower row). Error bars denote 95\% CI's of posterior means.

In Models 2a and 2b, we allow the slope of subjective SES to vary across countries. While the fixed parameters of the models are hardly affected by this change, 
model fit, however, improves substantially. A deviance test between Models 1a and $2 \mathrm{a}$ shows that the deviance decreases by 47.37 after adding the random slope for subjective SES and a parameter for the covariance between subjective SES and the random intercept, indicating a highly significant improvement of model fit with $d f=2, p<.001$. The same test for Models $1 \mathrm{~b}$ and $2 \mathrm{~b}$ yields a similar finding, with a deviance difference of $72.50, d f=2, p<.001$. Substantially, these tests indicate that there is variation across countries in the size of the association between subjective SES and the two health outcomes of self-rated health and psychological wellbeing. Furthermore, the small covariance parameters of subjective SES and the random intercept indicate that there is no association between the average health status/average psychological wellbeing in a country and the size of the subjective SES coefficient. This is confirmed in upper-row panels of Figure 3.3, which display the empirical Bayes regression lines of subjective SES on self-rated health (upper left) and psychological wellbeing (lower left).

The panels in the middle row of Figure 3.3 give us further insights into the variation of the subjective SES-health association across countries and provide a test of Hypothesis 1A. Hypothesis 1A had posited that the relationship between subjective SES and health exists in all countries of our sample. The panels in the middle row display caterpillar plots of the empirical Bayes residuals as obtained after fitting a random coefficient model (specifically, Models $2 \mathrm{a}$ and 2b) for the health outcomes, including subjective SES (random and fixed component), and age, sex, and marital status as control variables. For the right-hand panel in the middle row pertaining to self-rated health, we see that there is significant variation in the subjective SES-self-rated health association across countries, with the Philippines and the Netherlands showing the weakest effects of subjective SES on self-rated health, and the Nordic countries Denmark and Norway showing the strongest effect. The line towards the bottom of the panel indicates -1 times the upper bound of the 95 per cent confidence interval of the fixed coefficient of subjective $\mathrm{SES}^{2}$. Given the non-overlap of the confidence intervals of the empirical

\footnotetext{
${ }^{2}$ We are fitting a model

$$
Y_{i j}=\beta_{0}+\beta_{1 j} \text { subjective } \operatorname{SES}_{i j}+\beta_{2} \text { controls }_{i j}+u_{0 j}+u_{1 j}+e_{0 i j}
$$

where $u_{0 j}$ denotes the variation around the intercept $\beta_{0}$ and $u_{1 j}$ the slope variation around $\beta_{1 j}$. From this model, we obtain the empirical Bayes residuals $\hat{u}_{1 j}$, which are substantively the country-specific deviations from the fixed coefficient of subjective SES $\beta_{1 j}$. Hypothesis 1A posits that
}

$$
\beta_{1 j}+\hat{u}_{1 j}>0
$$

It follows that

$$
\hat{u}_{1 j}>-\beta_{1 j}
$$


Bayes residuals with this line, we can infer that the relationship of subjective SES on self-rated health is significantly different from zero in all countries under study. This finding gives support to Hypothesis 1A, which had posited exactly that. In the right-hand panel in the middle row, we see a similar pattern for psychological wellbeing: the subjective SES-psychological wellbeing association is statistically significant in all countries under study. The order of countries differs somewhat from the one found for the subjective SES-self-rated health association. In Norway and Latvia, the association between subjective SES and psychological wellbeing is strongest, while it is weakest in the Czech Republic and Slovenia. In sum, our analyses fully support Hypothesis 1A.

In Models 3a and 3b of Table 3.2, we additionally control for objective SES indicators, namely education, occupational prestige (ISEI), and household income. While the relationship between subjective SES and self-rated health is reduced (to .11) in Model 3a, it remains significantly different from zero. We also see clear and statistically significant gradients with respect to all objective SES indicators. The better educated and the ones with more prestigious jobs fare better in terms of self-rated health, and those with higher income enjoy better health than the poor. Those who have never held a job appear to have no health disadvantages when compared to those with an average ISEI score. A similar pattern arises in Model 3b, where psychological wellbeing is the outcome variable. Again, the coefficient of subjective SES drops slightly (to .11), but is still different from zero, as indicated by the $t$-statistic. The coefficients for education show that the relationship to psychological wellbeing is unexpectedly non-linear, with those with a medium degree reporting greater wellbeing than both those in the bottom and the top educational groups. For occupational prestige, we also see a positive relationship to wellbeing, and we can see that those who have never held a job in their lives report slightly worse psychological wellbeing than those with a job with an average ISEI score. The coefficients for household income reveal that reported wellbeing increases with each income quintile.

So far, our results show that the subjective SES-health relationship exists over and above the effects of objective SES in our sample of countries, for both selfrated general health and psychological wellbeing, but only for the fixed-effects, average coefficient. In order to ascertain whether this really holds in each and every country of our sample, we turn to the bottom row of Figure 3.3, which shows two caterpillar plots as obtained from Models $3 \mathrm{a}$ and $3 \mathrm{~b}$. The two panels of the bottom row of Figure 3.3 are similar to those of the middle row, but the

\footnotetext{
So in order to test that $\beta_{1 j}+\hat{u}_{1 j}$ is greater than 0 in all countries at the $99 \%$ level, the lower bound of the $95 \%$ CI of $\hat{u}_{1 j}$ must not overlap with -1 times the upper bound of the $95 \%$ CI of $\beta_{1 j}$.
} 
difference is that the caterpillar plots in the bottom row are based on Models $3 \mathrm{a}$ and $3 \mathrm{~b}$, which control for objective indicators of SES. Thus, the two panels in the bottom row of Figure 3.3 serve as a test of Hypothesis 1B, which had posited that subjective SES is positively related to health in all countries under study even after controlling for indicators of objective SES. We see that there is no overlap between the confidence intervals of the empirical Bayes estimates for each country and the dashed lines denoting the upper bounds of the 95 per cent confidence intervals of the fixed effect coefficients of subjective SES. This indicates that the relationship between subjective SES and both our health outcomes is greater than zero in all countries in our sample at conventional levels of statistical precision, supporting Hypothesis 1B. When comparing the caterpillar plots in Figure 3.3 across models, it shows that controlling for objective SES indicators has little impact on the order of countries. For both outcomes, the three countries with the strongest and the weakest associations remain the same after accounting for objective SES.

But how can the variation in the subjective SES-health relationship be explained? Hypothesis 2 posited that country affluence could play a crucial role, decreasing the importance of subjective SES for health outcomes. Table 3.3 tests this hypothesis in Models 4a and 4b. Model 4a reveals that there is a statistically significant interaction between GDP per capita and subjective SES for self-rated health, but not for psychological wellbeing (Model 4b). Figure 3.4 plots the predicted slopes for the subjective SES-self-rated health correlation at different levels of subjective SES and GDP per capita. Firstly, the plot reveals that the differences at different levels of GDP are substantially small and, secondly, that the results contradict Hypothesis 2. The more affluent a country is, the greater the health inequalities according to subjective SES. Thus, our findings do not support Hypothesis 2.

Hypothesis 3 posited that there is a cross-level interaction between income inequality and subjective SES: The greater the income inequality in a country, the stronger the relationship between subjective SES and health. Models 5a and $5 \mathrm{~b}$ of Table 3.3 test Hypothesis 3 and reveal that there such moderation. The coefficients are small and statistically not different from zero, indicating that the strength of the relationship between subjective SES and health in a country does not depend on its income distribution.

\subsubsection{Sensitivity analyses}

In order to assess the robustness of our results, we have conducted various sensitivity checks, which are presented in the Appendix of this Chapter. Firstly, we have re-estimated our random coefficient models via two-step OLS regression models. 
Table 3.3: Self-rated health and psychological wellbeing regressed on several predictors (random coefficient models), cross-level interactions

\begin{tabular}{|c|c|c|c|c|}
\hline & $\begin{array}{c}(4 a) \\
\text { Self-rated } \\
\text { general health }\end{array}$ & $\begin{array}{c}(4 \mathrm{~b}) \\
\text { Psychological } \\
\text { wellbeing }\end{array}$ & $\begin{array}{c}(5 \mathrm{a}) \\
\text { Self-rated } \\
\text { general health }\end{array}$ & $\begin{array}{c}\text { (5b) } \\
\text { Psychological } \\
\text { wellbeing }\end{array}$ \\
\hline Subjective SES & $\begin{array}{c}0.109^{* * *} \\
(17.87)\end{array}$ & $\begin{array}{c}0.109^{* * *} \\
(14.69)\end{array}$ & $\begin{array}{c}0.135^{* * *} \\
(6.03)\end{array}$ & $\begin{array}{c}0.0858^{* * *} \\
(3.36)\end{array}$ \\
\hline $\begin{array}{l}\text { Education (ref. low education) } \\
\text { Medium education }\end{array}$ & $\begin{array}{l}0.0886^{* * *} \\
(6.64)\end{array}$ & $\begin{array}{c}0.0374^{* *} \\
(2.65)\end{array}$ & $\begin{array}{c}0.0885^{* * *} \\
(6.63)\end{array}$ & $\begin{array}{c}0.0372^{* *} \\
(2.63)\end{array}$ \\
\hline High education & $\begin{array}{c}0.120^{* * *} \\
(6.88)\end{array}$ & $\begin{array}{c}0.00282 \\
(0.15)\end{array}$ & $\begin{array}{c}0.121^{* * *} \\
(6.89)\end{array}$ & $\begin{array}{c}0.00294 \\
(0.16)\end{array}$ \\
\hline ISEI & $\begin{array}{l}0.00201^{* * *} \\
\quad(5.12)\end{array}$ & $\begin{array}{c}0.00120^{* *} \\
(2.90)\end{array}$ & $\begin{array}{l}0.00202^{* * *} \\
(5.14)\end{array}$ & $\begin{array}{c}0.00120^{* *} \\
(2.92)\end{array}$ \\
\hline $\begin{array}{l}\text { Never worked } \\
\text { (ref. works/worked) }\end{array}$ & $\begin{array}{c}-0.00648 \\
(-0.29)\end{array}$ & $\begin{array}{c}-0.0569^{*} \\
(-2.49)\end{array}$ & $\begin{array}{c}-0.00690 \\
(-0.31)\end{array}$ & $\begin{array}{c}-0.0565^{*} \\
(-2.48)\end{array}$ \\
\hline $\begin{array}{l}\text { Income (ref. lowest quintile) } \\
\text { Second quintile }\end{array}$ & $\begin{array}{l}0.0818^{* * *} \\
(4.47)\end{array}$ & $\begin{array}{c}0.117^{* * *} \\
(6.13)\end{array}$ & $\begin{array}{l}0.0817^{* * *} \\
(4.47)\end{array}$ & $\begin{array}{c}0.117^{* * *} \\
(6.13)\end{array}$ \\
\hline Third quintile & $\begin{array}{c}0.0844^{* * *} \\
(4.57)\end{array}$ & $\begin{array}{c}0.189^{* * *} \\
(9.76)\end{array}$ & $\begin{array}{c}0.0842^{* * *} \\
(4.56)\end{array}$ & $\begin{array}{c}0.189^{* * *} \\
(9.76)\end{array}$ \\
\hline Fourth quintile & $\begin{array}{c}0.142^{* * *} \\
(7.46)\end{array}$ & $\begin{array}{c}0.207^{* * *} \\
(10.38)\end{array}$ & $\begin{array}{c}0.141^{* * *} \\
(7.45)\end{array}$ & $\begin{array}{c}0.207^{* * *} \\
(10.38)\end{array}$ \\
\hline Fifth quintile & $\begin{array}{c}0.191^{* * *} \\
(9.73)\end{array}$ & $\begin{array}{c}0.209^{* * *} \\
(10.14)\end{array}$ & $\begin{array}{c}0.191^{* * *} \\
(9.73)\end{array}$ & $\begin{array}{c}0.209^{* * *} \\
(10.13)\end{array}$ \\
\hline Income missing & $\begin{array}{c}0.135^{* * *} \\
(7.44)\end{array}$ & $\begin{array}{c}0.194^{* * *} \\
(10.14)\end{array}$ & $\begin{array}{c}0.134^{* * *} \\
(7.41)\end{array}$ & $\begin{array}{c}0.194^{* * *} \\
(10.13)\end{array}$ \\
\hline Age & $\begin{array}{c}-0.0158^{* * *} \\
(-36.31)\end{array}$ & $\begin{array}{c}-0.00117^{*} \\
(-2.55)\end{array}$ & $\begin{array}{c}-0.0158^{* * *} \\
(-36.27)\end{array}$ & $\begin{array}{c}-0.00118^{*} \\
(-2.57)\end{array}$ \\
\hline Female (ref. male) & $\begin{array}{c}-0.0412^{* * *} \\
(-3.95)\end{array}$ & $\begin{array}{c}-0.161^{* * *} \\
(-14.65)\end{array}$ & $\begin{array}{c}-0.0412^{* * *} \\
(-3.96)\end{array}$ & $\begin{array}{c}-0.161^{* * *} \\
(-14.65)\end{array}$ \\
\hline \multicolumn{5}{|l|}{ Marital status (ref. married/cohabiting) } \\
\hline Divorced & $\begin{array}{c}-0.0418^{*} \\
(-2.44)\end{array}$ & $\begin{array}{c}-0.178^{* * *} \\
(-9.77)\end{array}$ & $\begin{array}{c}-0.0420^{*} \\
(-2.46)\end{array}$ & $\begin{array}{c}-0.178^{* * *} \\
(-9.78)\end{array}$ \\
\hline Widowed & $\begin{array}{c}-0.0926^{* * *} \\
(-4.30)\end{array}$ & $\begin{array}{c}-0.180^{* * *} \\
(-7.99)\end{array}$ & $\begin{array}{c}-0.0925^{* * *} \\
(-4.30)\end{array}$ & $\begin{array}{c}-0.179^{* * *} \\
(-7.98)\end{array}$ \\
\hline Single & $\begin{array}{c}-0.0427^{* *} \\
(-2.94)\end{array}$ & $\begin{array}{c}-0.116^{* * *} \\
(-7.54)\end{array}$ & $\begin{array}{c}-0.0428^{* *} \\
(-2.95)\end{array}$ & $\begin{array}{c}-0.116^{* * *} \\
(-7.55)\end{array}$ \\
\hline GDP per capita (logged) & $\begin{array}{c}0.0746 \\
(1.43)\end{array}$ & $\begin{array}{c}-0.0224 \\
(-0.63)\end{array}$ & & \\
\hline GDP per capita $($ logged $) \times$ Subjective SES & $\begin{array}{c}0.0131^{*} \\
(1.99)\end{array}$ & $\begin{array}{c}0.00164 \\
(0.21)\end{array}$ & & \\
\hline Income inequality & & & $\begin{array}{c}-0.00320 \\
(-0.60)\end{array}$ & $\begin{array}{c}0.000549 \\
(0.16)\end{array}$ \\
\hline Income inequality $\times$ Subjective SES & & & $\begin{array}{c}-0.000818 \\
(-1.25)\end{array}$ & $\begin{array}{c}0.000694 \\
(0.93)\end{array}$ \\
\hline Intercept & $\begin{array}{c}1.888^{* * *} \\
(38.11)\end{array}$ & $\begin{array}{l}3.062^{* * *} \\
(83.37)\end{array}$ & $\begin{array}{c}1.987^{* * *} \\
(11.11)\end{array}$ & $\begin{array}{c}3.047^{* * *} \\
(25.48)\end{array}$ \\
\hline Variance (intercept) & $0.0575^{* * *}$ & $0.0258^{* * *}$ & $0.0612^{* * *}$ & $0.0262^{* * *}$ \\
\hline Variance (residual) & $0.747^{* * *}$ & $0.753^{* * *}$ & $0.747^{* * *}$ & $0.753^{* * *}$ \\
\hline Variance (subjective SES) & $0.000674^{* * *}$ & $0.00107^{* * *}$ & $0.000754^{* * *}$ & $0.00103^{* * *}$ \\
\hline Covariance (subjective SES, intercept) & 0.000402 & $-0.00243^{*}$ & 0.00100 & $-.00252^{*}$ \\
\hline Deviance & 76040.0 & 69589.0 & 76043.4 & 69588.2 \\
\hline$N$ countries & 27 & 26 & 27 & 26 \\
\hline$N$ & 29,799 & 27,191 & 29,799 & 27,191 \\
\hline
\end{tabular}




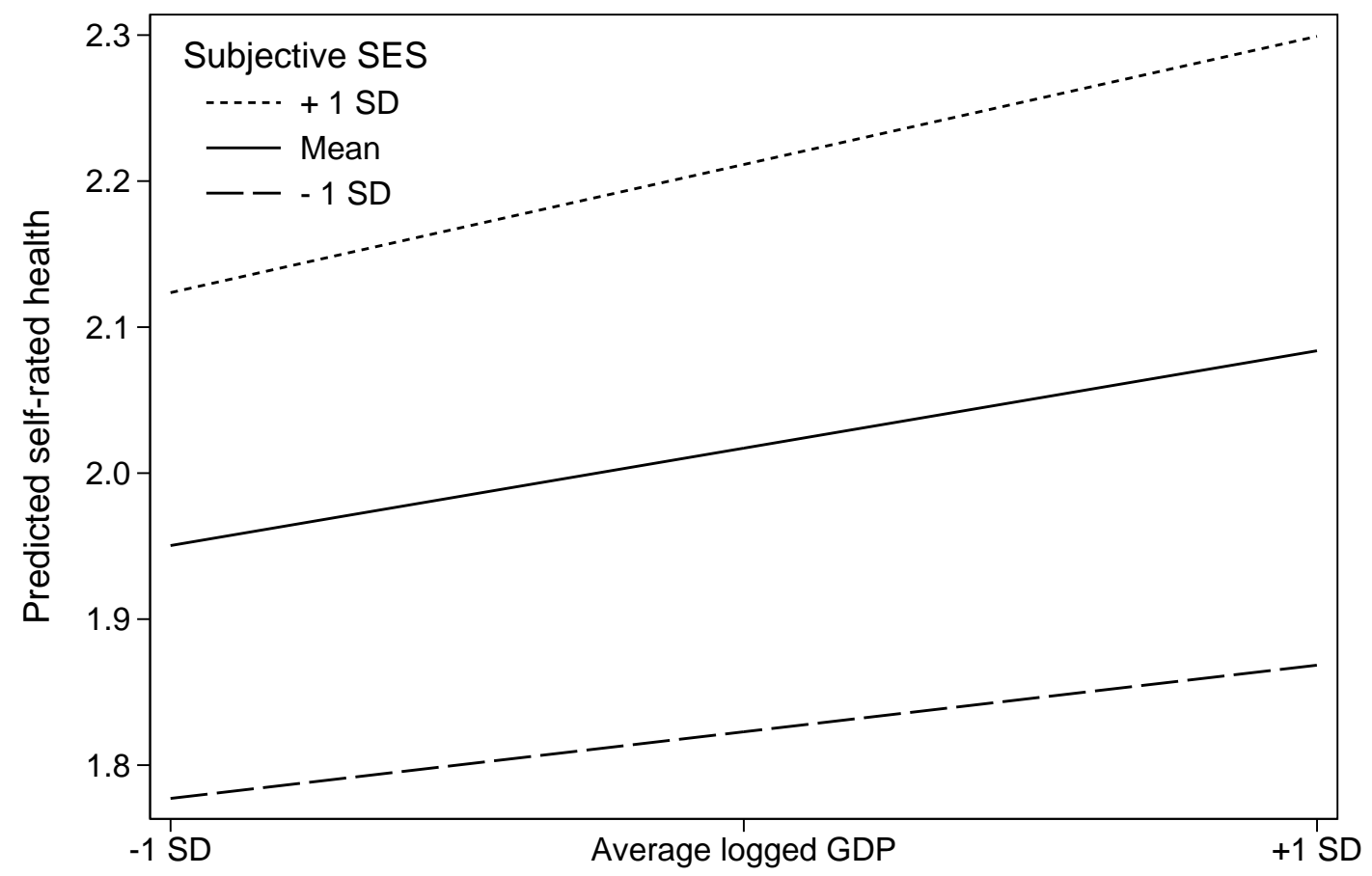

Figure 3.4: Plot of the GDP per capita $\times$ subjective SES interaction for self-rated general health

Recent simulations have suggested that estimates of variance components in random effects models are unreliable when the number of countries is small (Bryan and Jenkins, 2015). Bryan and Jenkins (2015) argue to have at least 25 countries for random effects models with linear outcomes. Although we are above that threshold with our sample of countries, this is only by a small margin. Another advantage of the two-step OLS regression approach is that fitting country-specific models amounts to including random slopes for all predictor variables in our models. The implicit assumption of the Models presented in Tables 3.2 and 3.3 is that subjective SES is the only predictor variable which varies in the strength of its relationship to the outcomes across countries. While this assumption is unlikely to be tenable, allowing more slopes to vary and introducing more variance components and covariance terms in our models easily leads to convergence problems. Thus, the two-step OLS regression approach also tests the robustness of our results when these assumptions are violated. Secondly, we have re-estimated all models with self-rated health as an outcome variable after having excluded France to demonstrate that our results are not dependent on the presence of France in the sample. Results show that our results prove to be robust to these additional checks. 


\subsection{Discussion}

Research has established a relationship between subjective socioeconomic status (SES) and health that appears to hold over and above the associations health has with objective indicators of SES. Drawing on data from 27 countries, we present the first cross-national study confirming this finding for two different health outcomes: self-rated overall health and psychological wellbeing. Subjective SES is significantly related to self-rated health and psychological wellbeing in all countries in our sample. This finding holds even after controlling for the objective SES markers of income, education, and occupational prestige.

Furthermore, we document significant variation across countries in the subjective SES-health relationship, with stronger relationships in countries, such as Norway, and a weaker association in the Philippines and the Netherlands. Thus, our results add to the emerging body of comparative research on social inequalities in health. Similar to many other studies (e.g. Brennenstuhl et al., 2012), it is difficult to explain patterns of cross-national variation in health inequalities. Hypothesized country-level moderation effects of country affluence and income inequality could not be found. Contrary to what we expected, we find an exacerbating effect of country affluence on the subjective SES gradient in self-rated health. The richer a country, the greater the effect of subjective SES on self-rated health. This can be interpreted in the light of Wilkinson and Pickett's (2010) idea that subjective status considerations are more important for health in more affluent countries than in countries where the fulfillment of basic needs is more important for health. Nonetheless, the questions remain whether, on the one hand, an effect of the size we find is clinically relevant and, on the other hand, why such an effect is not found for our second outcome variable psychological wellbeing, which should presumably be more sensitive to status considerations than a measure of general health.

The absence of a moderating effect of income inequality was also unexpected, as greater social inequality in a country could, in principle, make perceived low social status more painful. However, this notion could not be corroborated. The strength of the association between subjective SES and self-rated health, as well as psychological wellbeing, is independent of the income distribution in a country. This contradicts the findings of Wilkinson and Pickett (2008), who suggested that greater income inequality exacerbates health inequalities due to more status competition. While Wilkinson and Pickett (2008) examined average income and mortality rates in US counties, we put their explanation to a more stringent test, looking at subjective SES, self-rated health, and psychological wellbeing: three indicators much closer to the hypothesized status competition mechanism than average income and aggregate mortality. Future research trying to understand 
variation in the subjective SES-health gradient could consider examining cultural differences between countries (e.g. Hofstede, 2001), for instance the distinction between what has been called tight and loose cultures (Gelfand et al., 2011; Harrington and Gelfand, 2014). 'Tight' cultures are characterized by strong social norms and formal hierarchies, which might buffer the negative health effects of low subjective SES.

Nonetheless, there are a number of limitations of our study that need to be acknowledged. Given the reliance on cross-sectional and self-reported data, which is a widespread problem in cross-national research on health inequalities (Olafsdottir et al., 2013), it is difficult to make causal claims based on the findings at hand. However, previous longitudinal research has been able to establish that the subjective SES-health relationship can only partially be attributed to reverse causality (Garbarski, 2010; Nobles et al., 2013). Another aspect of endogeneity that could affect our findings is omitted variable bias. Previous research has speculated whether the relationship between health (especially self-reports) and subjective SES could be spurious, as both could be affected by an unmeasured individual characteristic like a personality trait (Singh-Manoux et al., 2005). However, a recent experimental study was able to show that the relationship between subjective SES and self-rated health was not affected by an experimental mood induction (Kraus et al., 2013), giving support to the notion that negative mood is not a confounder of the subjective SES-self-reported health relationship and strengthening the case for using self-reports of health in our study.

\section{Appendix}

This Appendix presents additional descriptive statistics and a variety of sensitivity analyses of the results presented in the main text of the Chapter.

\section{Country-level descriptive statistics}

Appendix Table A3.1 reports country-level descriptive statistics for the Gini coefficient and GDP per capita and gives individual-level sample sizes per country. 
Table A3.1: Descriptive statistics of country-level variables, $N=27$

\begin{tabular}{|c|c|c|c|}
\hline Country & Gini coefficient & GDP per capita & Sample size \\
\hline $\mathrm{AU}$ & 33.9 & $36,654.20$ & 1,280 \\
\hline $\mathrm{BE}$ & 25.1 & $36,877.00$ & 1,011 \\
\hline BG & 35.8 & $4,570.51$ & 776 \\
\hline $\mathrm{CH}$ & 30.2 & $55,005.90$ & 926 \\
\hline CL & 49.7 & $9,030.74$ & 1,155 \\
\hline $\mathrm{CZ}$ & 25.6 & $14,402.00$ & 1,393 \\
\hline $\mathrm{DE}$ & 30.3 & $37,321.80$ & 1,234 \\
\hline DK & 27.0 & $46,699.20$ & 1,062 \\
\hline $\mathrm{FI}$ & 25.5 & $38,921.70$ & 1,038 \\
\hline FR & 28.9 & $34,405.40$ & 2,461 \\
\hline HR & 27.6 & $10,711.30$ & 813 \\
\hline IL & 37.0 & $22,273.20$ & 871 \\
\hline JP & 30.5 & $36,160.80$ & 882 \\
\hline $\mathrm{KR}$ & 31.4 & $21,226.00$ & 1,284 \\
\hline LT & 36.4 & $9,566.36$ & 911 \\
\hline NL & 26.8 & $41,305.50$ & 1,024 \\
\hline $\mathrm{NO}$ & 22.2 & $64,534.00$ & 1,386 \\
\hline $\mathrm{PH}$ & 41.3 & $1,429.75$ & 998 \\
\hline PL & 29.7 & $10,387.40$ & 868 \\
\hline $\mathrm{PT}$ & 33.2 & $18,442.70$ & 779 \\
\hline $\mathrm{RU}$ & 45.2 & $6,633.07$ & 1,076 \\
\hline $\mathrm{SE}$ & 21.9 & $43,749.90$ & 796 \\
\hline SI & 24.2 & $19,147.80$ & 764 \\
\hline SK & 24.0 & $14,672.30$ & 845 \\
\hline $\mathrm{TR}$ & 37.5 & $8,413.32$ & 1,068 \\
\hline TW & 30.5 & $37,719.60$ & 840 \\
\hline $\mathrm{ZA}$ & 63.5 & $5,923.99$ & 2,111 \\
\hline Average & 32.4 & $25,414.28$ & 1,098 \\
\hline$S D$ & 9.2 & $17,234.10$ & - \\
\hline
\end{tabular}

Note: Gini from Solt (2009), GDP per capita from

World Bank (2014a). GDP per capita for

Taiwan taken from IMF (2012) 


\section{Subjective SES-health association based on country-specific OLS regression models}

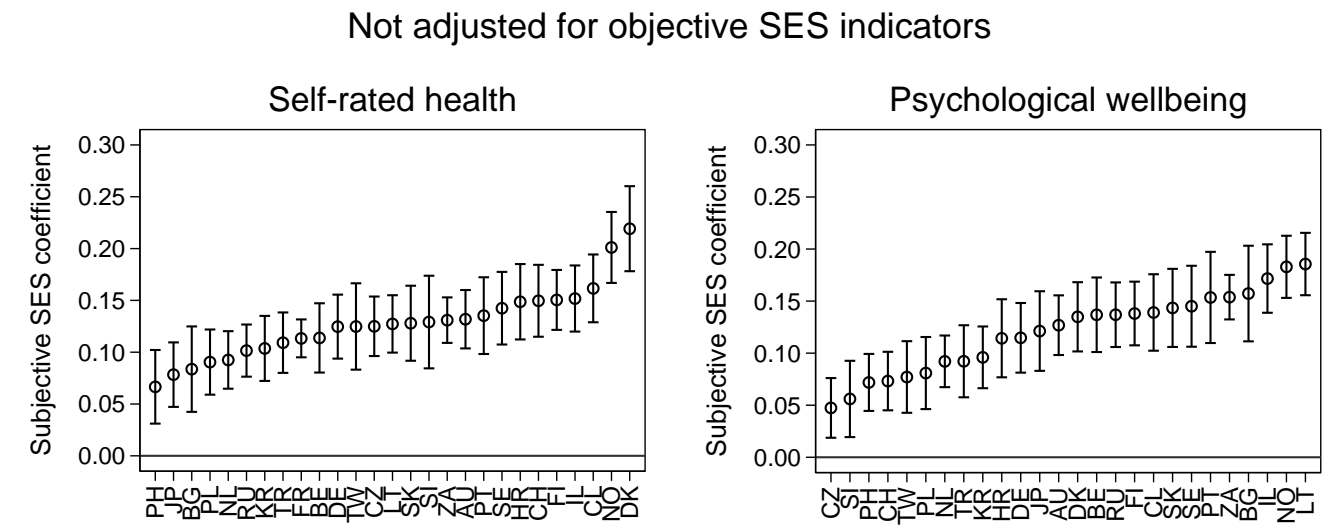

Adjusted for objective SES indicators
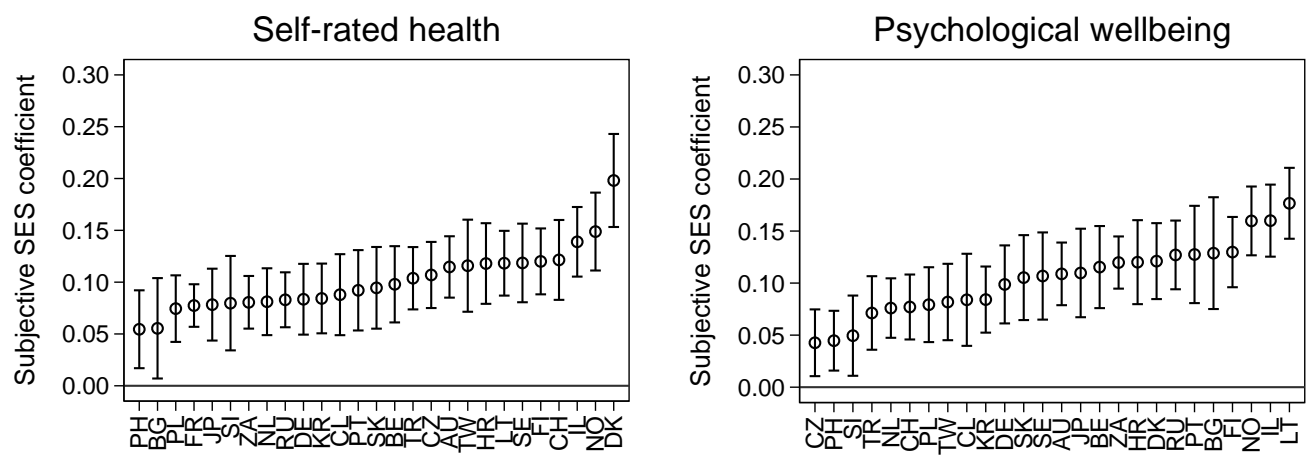

Figure A3.1: Association between subjective SES and health outcomes across countries, based on country-specific OLS regression models. Upper row: not adjusting for objective SES indicators. Lower row: adjusting for objective SES indicators.

Notes: Error bars denote 95\% CI's of OLS coefficients. All models control for age, sex, and marital status.

Figure A3.1 tests Hypotheses 1A and 1B using country specific OLS regression models instead of random coefficient models for the entire sample. The panels in the upper row of Figure A3.1 present the unstandardized regression coefficients of subjective SES along with their 95 per cent confidence intervals, stemming from country-specific OLS models which additionally controlled for age, sex, and marital status. It shows that the subjective SES coefficients are greater than zero at conventional levels of statistical precision in all countries of our sample for both outcome variables, thus supporting Hypothesis 1A. A comparison with the panels 
in the middle row of Figure 3.3 show that the ordering of countries is similar regardless of the estimation procedure.

The panels at the bottom of Figure A3.1 report the same parameters as those in the top row, however, they are now derived from models additionally controlling for objective indicators of SES education, occupational prestige, and household income. Again, the 95 per cent confidence intervals indicate that the subjective SES-health association can be found in all countries in our study, even when objective indicators of SES are accounted for. This finding supports Hypothesis 1B. Also, a comparison with the corresponding panels at the bottom of Figure 3.3 reveals that country order is similar for both approaches.

\section{Cross-level interactions estimated via the two-step approach}

Figure A3.2 reports tests of Hypotheses 2 and 3 using the two-step OLS regression approach. For each country sample and each outcome variable, an OLS regression model including subjective SES and controlling for the objective SES indicators, as well as age, sex, and marital status, was estimated. The unstandardized subjective SES coefficients were then regressed on GDP per capita and against the Gini coefficient. Results are reported in the scatterplots displayed in Figure A3.2. The panels in the top row of Figure A3.2 confirm the findings reported in Models 4a and 4b of Table 3.3, as well Figure 3.4. There is a positive correlation between country affluence and the strength of the subjective SES-self-rated health correlation, but not for psychological wellbeing, thus Hypothesis 2 is not supported. 
Interactions with GDP per capita
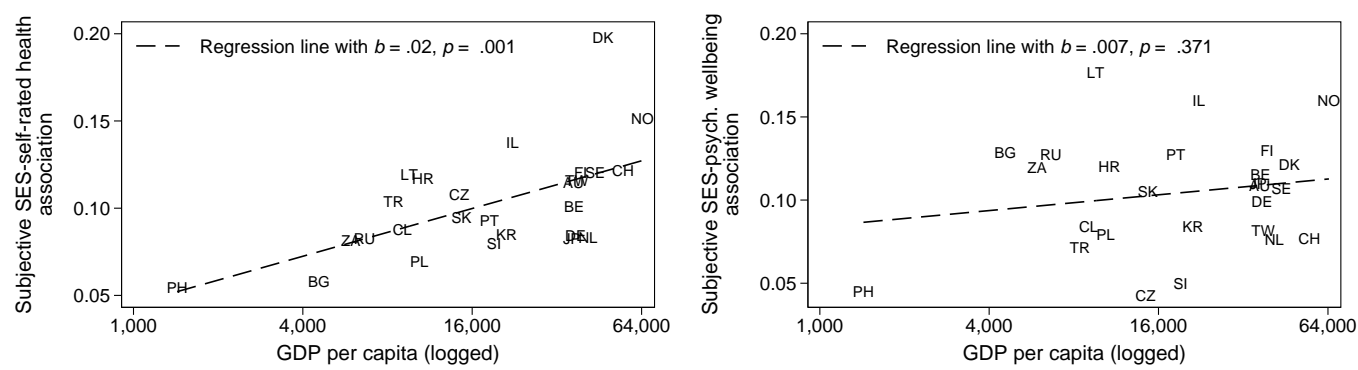

Interactions with income inequality
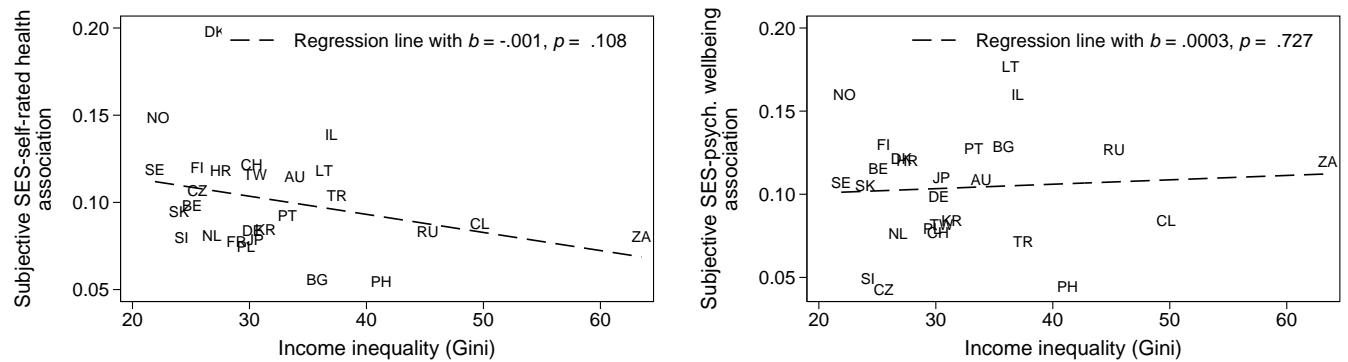

Figure A3.2: Scatterplots of subjective SES-health associations against countrylevel predictors, based on country-specific OLS models. Upper row: logged GDP per capita. Lower row: income inequality.

Note: All models control for education, occupational prestige, household income, age, sex, and marital status.

The panels in the bottom row of Figure A3.2 display another test of Hypothesis 3, which had suggested that the subjective SES-health relationship is stronger in less egalitarian countries. Identical to the results reported in Models $5 \mathrm{a}$ and $5 \mathrm{~b}$ of Table 3.3, no support for Hypothesis 3 is found, as no clear pattern emerges from the plots.

\section{Models for self-rated health excluding France}

Table A3.2 replicates the findings for self-rated health as reported in Tables 3.2 and 3.3 after excluding the French sample. The French sample has been excluded from the Models predicting psychological wellbeing. To show that our findings for self-rated health are not an artefact of including the French sample, we also report our findings after removing the French respondents. Table A3.2 shows that the results from our Models are robust against excluding France. 
Table A3.2: Self-rated health regressed on several predictors excluding the French sample

\begin{tabular}{|c|c|c|c|c|c|}
\hline & $\begin{array}{c}(1) \\
\text { Self-rated } \\
\text { general health }\end{array}$ & $\begin{array}{c}(2) \\
\text { Self-rated } \\
\text { general health }\end{array}$ & $\begin{array}{c}(3) \\
\text { Self-rated } \\
\text { general health }\end{array}$ & $\begin{array}{c}(4) \\
\text { Self-rated } \\
\text { general health }\end{array}$ & $\begin{array}{c}(5) \\
\text { Self-rated } \\
\text { general health }\end{array}$ \\
\hline Subjective SES & $\begin{array}{c}0.132^{* * *} \\
(40.65)\end{array}$ & $\begin{array}{c}0.133^{* * *} \\
(20.11)\end{array}$ & $\begin{array}{c}0.110^{* * *} \\
(16.63)\end{array}$ & $\begin{array}{c}0.112^{* * *} \\
(18.42)\end{array}$ & $\begin{array}{c}0.141^{* * *} \\
(6.33)\end{array}$ \\
\hline \multicolumn{6}{|c|}{ Education (ref. low education) } \\
\hline Medium education & & & $\begin{array}{c}0.0945^{* * *} \\
(6.63)\end{array}$ & $\begin{array}{c}0.0950^{* * *} \\
(6.67)\end{array}$ & $\begin{array}{c}0.0948^{* * *} \\
(6.65)\end{array}$ \\
\hline High education & & & $\begin{array}{c}0.121^{* * *} \\
(6.52)\end{array}$ & $\begin{array}{c}0.121^{* * *} \\
(6.50)\end{array}$ & $\begin{array}{c}0.121^{* * *} \\
(6.51)\end{array}$ \\
\hline ISEI & & & $\begin{array}{c}0.00190^{* * *} \\
(4.57)\end{array}$ & $\begin{array}{c}0.00188^{* * *} \\
(4.53)\end{array}$ & $\begin{array}{c}0.00189^{* * *} \\
(4.55)\end{array}$ \\
\hline Never worked (ref. works/w & rorked) & & $\begin{array}{c}-0.00944 \\
(-0.41)\end{array}$ & $\begin{array}{c}-0.00970 \\
(-0.42)\end{array}$ & $\begin{array}{c}-0.0101 \\
(-0.44)\end{array}$ \\
\hline \multicolumn{6}{|l|}{ Income (ref. lowest quintile) } \\
\hline Second quintile & & & $\begin{array}{c}0.0759^{* * *} \\
(3.94)\end{array}$ & $\begin{array}{c}0.0760^{* * *} \\
(3.94)\end{array}$ & $\begin{array}{l}0.0759^{* * *} \\
(3.93)\end{array}$ \\
\hline Third quintile & & & $\begin{array}{c}0.0841^{* * *} \\
(4.31)\end{array}$ & $\begin{array}{c}0.0842^{* * *} \\
(4.31)\end{array}$ & $\begin{array}{c}0.0840^{* * *} \\
(4.30)\end{array}$ \\
\hline Fourth quintile & & & $\begin{array}{c}0.135^{* * *} \\
(6.71)\end{array}$ & $\begin{array}{c}0.135^{* * *} \\
(6.71)\end{array}$ & $\begin{array}{c}0.135^{* * *} \\
(6.71)\end{array}$ \\
\hline Fifth quintile & & & $\begin{array}{c}0.186^{* * *} \\
(8.93)\end{array}$ & $\begin{array}{c}0.186^{* * *} \\
(8.93)\end{array}$ & $\begin{array}{c}0.186^{* * *} \\
(8.94)\end{array}$ \\
\hline Income missing & & & $\begin{array}{c}0.134^{* * *} \\
(6.97)\end{array}$ & $\begin{array}{c}0.136^{* * *} \\
(7.03)\end{array}$ & $\begin{array}{c}0.135^{* * *} \\
(7.00)\end{array}$ \\
\hline Age & $\begin{array}{c}-0.0176^{* * *} \\
(-38.75)\end{array}$ & $\begin{array}{c}-0.0175^{* * *} \\
(-38.59)\end{array}$ & $\begin{array}{c}-0.0163^{* * *} \\
(-35.29)\end{array}$ & $\begin{array}{c}-0.0164^{* * *} \\
(-35.33)\end{array}$ & $\begin{array}{c}-0.0163^{* * *} \\
(-35.28)\end{array}$ \\
\hline Female (ref. male) & $\begin{array}{c}-0.0494^{* * *} \\
(-4.52)\end{array}$ & $\begin{array}{c}-0.0493^{* * *} \\
(-4.51)\end{array}$ & $\begin{array}{c}-0.0437^{* * *} \\
(-3.95)\end{array}$ & $\begin{array}{c}-0.0436^{* * *} \\
(-3.94)\end{array}$ & $\begin{array}{c}-0.0436^{* * *} \\
(-3.95)\end{array}$ \\
\hline \multicolumn{6}{|c|}{ Marital status (ref. married/cohabiting) } \\
\hline Divorced & $\begin{array}{c}-0.0537^{* *} \\
(-2.93)\end{array}$ & $\begin{array}{c}-0.0515^{* *} \\
(-2.81)\end{array}$ & $\begin{array}{c}-0.0428^{*} \\
(-2.33)\end{array}$ & $\begin{array}{c}-0.0424^{*} \\
(-2.31)\end{array}$ & $\begin{array}{c}-0.0426^{*} \\
(-2.33)\end{array}$ \\
\hline Widowed & $\begin{array}{c}-0.120^{* * *} \\
(-5.29)\end{array}$ & $\begin{array}{c}-0.120^{* * *} \\
(-5.30)\end{array}$ & $\begin{array}{c}-0.0960^{* * *} \\
(-4.24)\end{array}$ & $\begin{array}{c}-0.0963^{* * *} \\
(-4.25)\end{array}$ & $\begin{array}{c}-0.0962^{* * *} \\
(-4.25)\end{array}$ \\
\hline Single & $\begin{array}{c}-0.0436^{* *} \\
(-2.81)\end{array}$ & $\begin{array}{c}-0.0425^{* *} \\
(-2.73)\end{array}$ & $\begin{array}{c}-0.0421^{* *} \\
(-2.71)\end{array}$ & $\begin{array}{c}-0.0420^{* *} \\
(-2.71)\end{array}$ & $\begin{array}{c}-0.0422^{* *} \\
(-2.73)\end{array}$ \\
\hline GDP per capita (logged) & & & & $\begin{array}{c}0.0723 \\
(1.35)\end{array}$ & \\
\hline GDP per capita (logged) $\times$ & Subjective SES & & & $\begin{array}{c}0.0154^{*} \\
(2.39)\end{array}$ & \\
\hline Income Inequality & & & & & $\begin{array}{c}-0.00307 \\
(-0.57)\end{array}$ \\
\hline Income Inequality $\times$ Subjec & tive SES & & & & $\begin{array}{c}-0.000930 \\
(-1.44)\end{array}$ \\
\hline Intercept & $\begin{array}{c}2.069^{* * * *} \\
(40.76) \\
\end{array}$ & $\begin{array}{c}2.061^{* * *} \\
(41.01)\end{array}$ & $\begin{array}{c}1.881^{* * *} \\
(35.38) \\
\end{array}$ & $\begin{array}{c}1.887^{* * * *} \\
(36.39) \\
\end{array}$ & $\begin{array}{c}1.981^{* * *} \\
(10.76) \\
\end{array}$ \\
\hline Variance (intercept) & $0.0649^{* * *}$ & $0.0635^{* * *}$ & $0.0645^{* * *}$ & $0.0602^{* * *}$ & $0.0638^{* * *}$ \\
\hline Variance (residual) & $0.776^{* * *}$ & $0.774^{* * *}$ & $0.765^{* * *}$ & $0.765^{* * *}$ & $0.765^{* * *}$ \\
\hline Variance (subjective SES) & & $0.000839^{* * *}$ & $0.000792^{* * *}$ & $0.000597^{* * *}$ & $0.000715^{* * *}$ \\
\hline Covariance (subjective SES & , intercept) & 0.00186 & 0.00145 & 0.000581 & 0.00128 \\
\hline Deviance & 70380.9 & 70337.1 & 70046.9 & 70040.6 & 70044.8 \\
\hline$N$ countries & 26 & 26 & 26 & 26 & 26 \\
\hline$N$ & 27,191 & 27,191 & 27,191 & 27,191 & 27,191 \\
\hline
\end{tabular}




\section{Chapter 4}

\section{Income and Income Inequality as Social Determinants of Health: Do Social Comparisons Play a Role? ${ }^{1}$}

\subsection{Introduction}

The effects of social conditions on individual health have long been acknowledged. Earlier research has conclusively shown how both social relationships and socioeconomic status (SES) have positive effects on a large variety of health outcomes. Individuals who are better socially integrated benefit from better health than those who are less socially integrated (Holt-Lunstad et al., 2010), and individuals with a higher SES exhibit better health than their low-SES counterparts (Elo, 2009). Research has further established that not only factors at the individual level affect health, but also contextual-level conditions. The recent debate as to how greater income inequality in a society can diminish the health of individuals in it is an example for such contextual influences on health. How such social antecedents operate to affect health and disease, however, is still not fully understood (Elo, 2009; Thoits, 2011).

Prominent explanatory approaches for these relationships have focused on the

\footnotetext{
${ }^{1}$ This Chapter is co-authored by Melinda Mills and Rafael Wittek. A slightly different version of this Chapter appeared in the European Sociological Review (Präg et al., 2014). Earlier versions of this Chapter were presented at the RC 28 Spring Meeting 2011 in Essex, the ECSR/EQUALSOC summer school 2011 in Trento, the ECSR conference 2011 in Dublin, and the Dutch Sociological Association meeting 2011 in Ghent. The authors thank the discussants at these occasions, the three anonymous ESR reviewers, as well as Richard Wilkinson and Jason Beckfield for detailed and constructive comments on drafts of the paper.
} 
material living conditions of individuals and societies and psychosocial mechanisms. Material living conditions such as strenuous physical labor or less health care access, have long been proposed as reasons for the poorer health of the lower social strata. With respect to the negative income inequality-health correlation, the so-called 'neo-materialist' approach blamed an underinvestment into human, physical, and cultural capital occurring in high-inequality societies (Davey Smith, 1996; Lynch et al., 2004). Societies with greater income inequality have fewer collective resources to invest in the educational, the medical, and cultural infrastructure, which in turn hurts health and stretches the social fabric.

Recently, researchers have turned to psychosocial factors to explain links between SES and health (Schnittker and McLeod, 2005). One of those factors, social comparisons, is assumed to be a key mechanism linking social conditions and health. For instance, comparing one's own health or health behaviors can have important effects on one's health assessments and future health behaviors (Thoits, 2011). Individuals receive guidance in terms of behavior and norms when comparing their health and health behaviors to others' health and lifestyles. Comparing with others can aid in the interpretation of physical symptoms, help adapting to health threats, and provide social validation for personal health behaviors (Suls, 2003).

Conversely, a type of social comparison process that has been suggested to lead to negative health effects is the comparison of social status. Social status comparisons are assumed to underlie two of the most prominent phenomena in research on societal determinants of health, the socioeconomic gradient in health (Marmot, 2004) and the negative inequality-health correlation (Wilkinson and Pickett, 2010). The socioeconomic gradient in health refers to the oft-replicated finding that in developed countries health disparities between the different socioeconomic strata arise, with those better off economically exhibiting better health than their counterparts from lower socioeconomic strata. The negative income inequality-health correlation refers to the finding that population health in those developed societies is worse where income inequality is higher. Some researchers have attributed these phenomena to a common core, namely the existence of status hierarchies in societies and the status competition that takes place in these hierarchies (Marmot, 2004; Wilkinson and Pickett, 2010).

According to this explanation, individuals are assumed to compete for status and prestige in social hierarchies, and being unsuccessful in this status competition, i.e. receiving negative appraisals of one's status, has been shown to lead to stress (Dickerson and Kemeny, 2004). Chronic stress, in turn, is related to all sorts of negative health outcomes (Sapolsky, 2004). Thus, being on a lower or higher rung of the social ladder leads to possible negative appraisals of one's status, which 
leads to stress and illnesses, and a socioeconomic gradient in health and illness emerges (Marmot, 2004). Also, living in a society with more income inequality, which equates to being on a social ladder where the rungs are farther apart, should result in more status competition and more negative appraisals of one's status. From this emerges a negative correlation between income inequality and average health. This could either affect all members of a society to the same extent (as suggested by Wilkinson and Pickett, 2010) or predominately those at the bottom of the income distribution (Lancee and Van de Werfhorst, 2012).

If these presumed causal chains were true, individuals who would be more sensitive to status competition should exhibit greater health effects. Social psychological research has shown that individuals vary in their propensity to engage in social comparisons (Buunk and Gibbons, 2007). Furthermore, it has been shown that those who are more prone towards comparisons are also more likely to be affected by the results of such comparisons (Buunk and Gibbons, 2007). In our study, we will focus on one form of status comparisons, namely income comparisons. We will proceed from the assumptions that income is an important status marker in contemporary societies (Marmot, 2004) and that the importance individuals attribute to income comparisons reflect their social comparison orientation and their sensitivity to status competition. We will utilize this income comparison orientation to test its moderating effect on the relationships between relative income, income inequality, and health.

This Chapter will extend existing research in three distinct ways. Firstly, we make use of a novel approach to test an assumption underlying a mechanism proposed to be responsible for both the negative health effects of low SES and income inequality, namely social comparisons of one's social status. According to our knowledge, this has never been tested before. We draw on social-psychological findings about differences in the propensity to engage in and the sensitivity towards social comparisons and this allows us to overcome the practical problems associated with reliably and validly measuring social comparisons. Earlier research on status comparisons as a linking mechanism has relied on imposing the assumption that individuals compare their incomes to demographically similar groups. Secondly, by focusing on a potential common cause we contribute to linking the up to now largely separate debates on health effects of income inequality and health inequalities (Beckfield et al., 2013; Wilkinson and Pickett, 2008). Thirdly, we aim at giving a comprehensive view of health as a psychophysical entity by drawing on two distinct indicators of health, namely general health and psychological wellbeing, both measured via self-reports. By using these two indicators as our outcome variables, we are able to distinguish between general and mental health effects of income and income inequality. 
Exploiting the third wave of the European Social Survey (ESS, 2006/07), our study analyzes the relationship between income inequality, income comparisons, and health for 18,356 Europeans in paid work from 23 countries.

\subsection{Theoretical background}

\subsubsection{The socioeconomic gradient in health}

The finding of an SES gradient in health, in the sense that those of higher socioeconomic status enjoy better health than those in lower positions of the social ladder, is a nearly universal one: Historical sources show that there has been a socioeconomic gradient in health in ancient Greece, Egypt, and China (Krieger et al., 1997). Comparative sources reveal that a socioeconomic gradient in health can nowadays be found in all countries (Beckfield et al., 2013; Elo, 2009; Mackenbach et al., 2005), irrespective of public health care provision and welfare regimes. Furthermore, the gradient has been documented not only for self-rated health and life expectancy, but also for a wide range of health outcomes.

Research on the SES-health gradient has proposed a whole range of causal pathways linking one's economic conditions to health, such as health behaviors, poverty, access to and quality of health care; however, these are seen as incomplete (Elo, 2009). Status differences are one of the many explanatory factors proposed and that have received a substantial amount of attention in the debate. Status in this context refers to distinction in valued aspects and does not necessarily entail a high SES (Brennan and Pettit, 2004; Frank, 1985). After an income threshold beyond material hardships has been passed, additional income does not buy better health - it becomes a marker of status (Marmot, 2004). In line with the gains in happiness that are brought about by a higher relative income (Clark et al., 2008), individuals are concerned about their position in the hierarchy. This corresponds to an evolutionary account of the emergence of status seeking (Marmot, 2004) and to links between hierarchies and stress and illness encountered in non-human primates (Sapolsky, 2005; Shively, 2000). Furthermore, the status competition explanation is in line with recent findings about subjective socioeconomic status: Subjective SES - the subjective assessment of one's own socioeconomic status in a society - has been shown to be crucially related to health outcomes Schnittker and McLeod (2005). Believing that one has a high socioeconomic position is beneficial for health irrespective of one's actual SES measured in an objective way. Subjective SES inherently involves social comparisons: one has to size up peers to gain an impression of one's own ranking in the social hierarchy. Evidence for the effect of subjective SES is strong: In an experimental prospective study, Cohen et al. 
(2008) were able to show that it was the subjective assessment of SES and not the objective indicators that predicted whether participants developed a common cold when exposed to a common cold virus.

In line with existing evidence (Mackenbach et al., 2008; Lorant et al., 2003), we suggest that there is a socioeconomic gradient in self-rated health and psychological wellbeing.

H1: Relative income is positively related to self-rated health and psychological wellbeing.

\subsubsection{The negative income inequality-health correlation}

Evidence of a zero-order correlation between income inequality and population health outcomes in developed societies has been presented since the late 1970's (Rodgers, 1979), but it was not until Wilkinson's (1992) contribution that the debate gained momentum. Wilkinson (1992) suggested that there was a -.86 countrylevel correlation between income inequality and life expectancy. Numerous replications followed, and probably the most extensive narrative review by Wilkinson and Pickett (2006) counted 139 studies until then. Studies, however, vary strongly in their methodological quality; especially early studies largely relied on aggregatelevel data with only few data points. Thus, narrative reviews like Wilkinson and Pickett (2006), Pickett and Wilkinson (2015), and Lynch et al. (2004) vary in their conclusions. Lynch and colleagues refute the idea of any meaningful correlation between income inequality and health and argue for the neo-materialist account of a spurious association between inequality and health, and Wilkinson and Pickett insist on a causal relationship. Also, recent high-quality studies only provide mixed evidence. Kondo et al.'s (2009) meta-analysis of earlier multilevel studies showed a correlation between income inequality and health. This correlation, however, was substantially attenuated when unmeasured characteristics of areas with high income inequality were accounted for. In a similar vein, fixed-effects analyses of macro-level data sets attributed the correlation between income inequality and health fully to unobserved time-constant country characteristics (Beckfield, 2004) or could find such a correlation only in low- and middle-income countries (Pop et al., 2013).

Based on theoretical reasoning and the bulk of the existing evidence, we suggest that there is a negative correlation between income inequality in a country and health:

H2: Income inequality is negatively correlated with self-rated health and psychological wellbeing. 


\subsubsection{Social comparisons and psychobiological reactions to hierarchies}

Social comparisons are assumed to be an innate human activity that evolved over evolutionary time. To match with potential competitors is an ability of high adaptive value that has been recognized in many species (Gilbert et al., 1995). Social comparisons form the foundations of self-knowledge (Fiske, 2011) and can satisfy the basic human need to feel competent by letting people know whether their opinions are correct and what their abilities allow them to do (Festinger, 1954). Also, social comparisons have long been a focal interest of classical sociology, for instance, Veblen's (1899) notion of conspicuous consumption, which suggested that social status is communicated to strangers via wasteful ways of spending money.

Social comparison theory has had sizable impact on research on health and health behaviors (Buunk and Gibbons, 1997; Suls, 2003). Individuals use the attitudes, beliefs, and behaviors of others as benchmarks for evaluating their own attitudes, beliefs, and behaviors, and usually shift their own to match those of the group. Furthermore, norms about health behaviors are acquired via social comparison processes, for instance, through the use of alcohol and cigarettes, seeking health care and counseling, adherence to treatment regimes, and to attend to diet (Thoits, 2011).

In the case of status hierarchies, social comparisons of status positions can have different effects. Perceiving oneself to be better than others is beneficial for selfesteem, positive affect, and it reduces anxiety. Negative results of comparisons diminish self-esteem, produce negative affect, and can cause stress (Buunk and Gibbons, 1997). In the case of an individual with low status, such as a person with little income, income comparisons will most likely lead to stress (Marmot, 2004). In the case of income inequality, people living in areas with high rather than low income inequality are more concerned about how they compare to others (status anxiety) and feel deprived, marginalized, and angry as a result (relative deprivation) (Wilkinson and Pickett, 2010). Threats to one's social esteem, value, and status have been shown to be very salient for creating stress (Dickerson and Kemeny, 2004).

Therefore, stress is linked to negative health effects in two ways. Firstly, there is a direct connection via physiological pathways. Stressed individuals react with a fight-or-flight response: energy is mobilized via putting out glucocorticoides to exercise muscles, and other, non-essential processes for fighting or fleeing are deferred, such as digestion, growth, inflammation, and tissue repair, and immune function is inhibited (Sapolsky, 2005). While this is functional in the case of immediate danger, studies have shown that these physiological processes taking 
place in stressful situations are dangerous when experienced chronically. Secondly, there is an indirect connection between stress and bad health in the sense that individuals might engage in unhealthy behaviors, such as smoking or overeating. These are forms of relaxation and pleasure that can serve to regulate the mood of the disadvantaged (Pampel et al., 2010).

Whereas early research assumed that social comparisons largely depend on situational factors (Mussweiler, 2003) and not on personality, recent research was able to show that individuals vary in their propensity to engage in social comparisons (Buunk and Gibbons, 2007). Social comparisons can be very functional in many situations. For example, a strong social comparison orientation has been shown to serve an adaptive function that enhances subjective wellbeing among the elderly (Frieswijk et al., 2007): Social comparisons provided older adults with information that allowed them to make adjusted assessments of their own situation. Research has also shown that results of such comparisons have a stronger impact on those who have a stronger social comparison orientation (Buunk and Gibbons, 2007). In our study, we will focus on an important and easily measurable aspect of social comparison orientation, namely income comparison orientation. An earlier study was able to show that income comparisons were less likely to be important the higher one's household income, although the effect was small and contradicted by a non-linear effect of education on income comparisons (Clark and Senik, 2010), which provided support to the notion that income comparison orientation is a personality trait.

In sum, distressing comparison processes have been suggested to be at the core of the socioeconomic gradient in health and the negative effects of income inequality on health. Previous research on the SES-health link did not consider that there are considerable personality differences in social comparison orientation, as shown by recent advances in psychological research on personality and social comparisons. We posit that individuals with a greater propensity to compare their incomes should suffer more in countries with greater income inequality and when their relative income is lower. In order to test whether comparison processes are truly key in explaining the socioeconomic gradient in health and the inequality-health association, we hypothesize that those individuals who are more sensitive towards comparison processes due to a greater income comparison orientation should experience greater health effects of relative income and of income inequality:

H3: Income comparison orientation moderates the effect of relative income on self-rated health and psychological wellbeing in the sense that those respondents with a stronger income comparison orienta- 
tion will experience stronger relative income effects.

H4: Income comparison orientation moderates the effect of income inequality on self-rated health and psychological wellbeing in the sense that those respondents with a stronger income comparison orientation will experience stronger income inequality effects.

\subsection{Data and methods}

\subsubsection{Data}

This study uses the third round of the European Social Survey (ESS, Jowell et al., 2007), a large-scale survey conducted in 25 European countries in 2006/07, namely Austria (AT), Belgium (BE), Bulgaria (BG), Switzerland (CH), Cyprus (CY), Germany (DE), Denmark (DK), Estonia (EE), Spain (ES), Finland (FI), France (FR), the United Kingdom (UK), Hungary (HU), Ireland (IE), Latvia (LV), The Netherlands (NL), Norway (NO), Poland (PL), Portugal (PT), Romania (RO), Russia (RU), Sweden (SE), Slovenia (SI), Slovakia (SK), and Ukraine (UA). Cyprus and Latvia had to be excluded from our study due to differences in the measurement of the income comparison variable. Thus, our analyses are based on 23 countries. In all countries, random probability samples were drawn, following standardized procedures and strict quality controls to ensure representativeness and comparability of the data. Each round of the ESS comprises a core questionnaire on a range of basic topics, such as demographics and health status, and rotating modules of questions probing for more specialized topics. The third round fielded a module titled "Personal and Social Wellbeing" (Huppert et al., 2009), which specifically contained questions on income comparisons and psychological wellbeing, making this wave of the ESS especially useful for our purposes.

Due to the fact that our focal independent variable, the subjective importance of income comparisons, was collected only for respondents who are active on the labor market (employed, self-employed, and family workers), our analyses are restricted to respondents currently in paid work. To ensure that outliers in terms of age do not affect our results, we removed respondents older than 70 years of age. Furthermore, we removed respondents with missing values on one of our study variables. This results in a sample size of 18,356 complete cases. Sample sizes per country are reported in Appendix Table A4.1. 


\subsubsection{Outcome variables}

Our analysis makes use of two dependent variables. Psychological wellbeing is based on a short version of the Center for Epidemiologic Studies Depression Scale (CES-D; Radloff, 1977). Despite being a scoring rather than diagnostic measure, the CES-D is a strong predictor for depression without covering all potential symptoms of depression. Of the original twenty CES-D items, eight items are included in the questionnaire, probing whether respondents felt, for instance, lonely, sad, or depressed in the last week. Four response options were provided, ranging from 'None or almost none of the time,' (0) to 'All or almost all of the time' (3). Responses were reverse coded, summed, and divided by eight, resulting in a composite variable ranging from 0 to 3 , so that higher values indicate a higher degree of psychological wellbeing. The scale exhibits a high degree of internal consistency (Cronbach's alpha $=.80$ ) and an earlier multi-group confirmatory factor analysis showed the validity of the scale for cross-national comparison (Van de Velde et al., 2010b). Furthermore, it has already been successfully applied in cross-nationally comparative studies (Huijts et al., 2013). The intraclass correlation coefficient $(I C C)$ of psychological wellbeing is .08 , which is modest by conventional standards.

Self-rated overall health is measured with a single item: 'How is your health in general?' Response options ranged from 'very bad' (0) to 'very good' (4). Selfrated health is a general assessment of one's health status, not connected to any specific illness, but covering largely physical and functional aspects of health (Idler et al., 1999). It has been shown to predict mortality and morbidity and has high test-retest reliability in a number of studies (Idler and Benyamini, 1997). Furthermore, this variable has been recommended by the World Health Organization for comparative research (De Bruin et al., 1996) and a large number of researchers have followed this advice (Hildebrand and Van Kerm, 2009; Huijts et al., 2010b), especially in the comparative study of health disparities (Gesthuizen et al., 2012; Mackenbach et al., 2008). Research has also shown that different socioeconomic groups evaluate their health in comparable ways (Burström and Fredlund, 2001) and that the associations between objective health indicators and self-perceived health are largely similar across countries (Bardage et al., 2005). Clustering of self-rated health within countries is moderate by conventional standards, with an $I C C$ of .13 .

Means and standard deviations of the variables are reported in Table 4.1, along with descriptive statistics for all other individual-level variables. The correlation between the two outcome variables is $r=.35$, reflecting that they are two interrelated, yet distinct aspects of health. 
Table 4.1: Descriptive statistics of individual-level variables, $N=18,356$

\begin{tabular}{lcccc}
\hline Variable & Mean & Std. dev. & Min. & Max. \\
\hline Psychological wellbeing & 2.33 & 0.46 & 0 & 3 \\
Self-rated overall health & 2.95 & 0.78 & 0 & 4 \\
& & & & \\
Female & 0.49 & - & 0 & 1 \\
Married/cohabiting & 0.56 & - & 0 & 1 \\
Age (centered) & 0.00 & 12.10 & -26.7 & 28.3 \\
& & & & \\
Income comparison orientation (centered) & 0.00 & 1.85 & -2.3 & 3.7 \\
Education in years (centered) & 0.00 & 3.63 & -13.6 & 9.4 \\
\hline
\end{tabular}

\subsubsection{Predictor variables}

Importance of income comparisons was measured by the question 'How important is it for you to compare your income with other people's incomes?' Original response options ranged from 'Not at all important' (0) to 'Very important' (6), with no labels for the response options in between. Despite being a measure that has not yet been used in research on health inequities, Clark and Senik (2010) were able to demonstrate construct validity by showing its negative correlations to a wide range of wellbeing measures, something we would expect from theory (Kasser and Ryan, 1993). The left panel of Figure 4.1 shows the average income comparison orientation by income quintiles, and, when looking at the point estimates, a slight negative relationship to income can be seen: Those at the bottom of the income distribution are more likely to engage in income comparisons than those at the top. The confidence intervals, however, show that the differences between income groups are statistically not significant. The right panel of Figure 4.1 presents country averages in income comparison orientation. It shows that Eastern European exhibit the greatest average income comparison orientation, whereas the wealthier countries show lower values.

Relative household income was included as a set of dummy variables representing country-specific income quintiles, with the highest (fifth) quintile serving as the reference category. Specifically, the questionnaire asked all respondents for total household net income from all sources. Response options were sets of income ranges which slightly varied over countries. Due to the fact that income information enters the models in the form of country-specific quintile dummy indicators, this does not have a substantial effect on our results. By using the mid-points of income categories, a metric household income variable was created, which was equivalized by dividing it by the square root of the number of household members. 


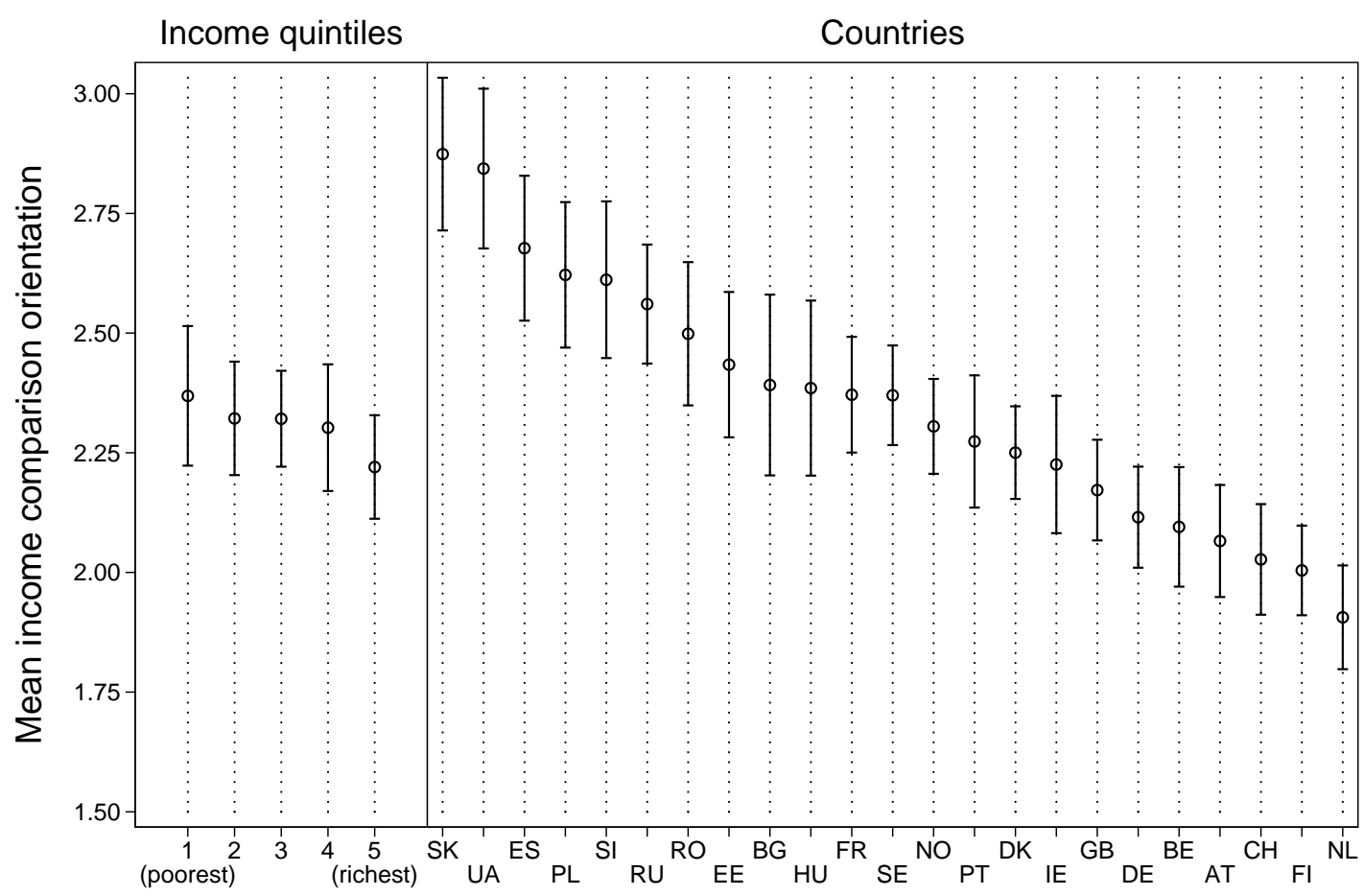

Figure 4.1: Average income comparison orientation by income quintile (left panel) and by country (right panel)

Notes: Error bars denote 95\% CI's. 95\% CI's in the left panel are based on cluster-robust standard errors.

This brings household income to the individual level and adjusts household income for the economies of scale that arise when individuals live together. Controlling for individual income when assessing the inequality-health relationship is crucial, as the nonlinear relationship between income and health on the individual level (every additional Euro will improve health only to a diminishing extent) can create a macro-level correlation between income inequality and health (Gravelle, 1998). With total income being constant, a more equal income distribution should yield better average health.

Income inequality was measured on the country-level via the Gini coefficient of net household income. The Gini coefficient ranges between 0 and 100, with 0 indicating an income distribution where every household has exactly the same income and 100 denoting that there is perfect inequality in the distribution (one household receives all income, while all other households receive nothing). Gini coefficients were obtained from the Eurostat database 2011 for the year that the ESS data were collected in that country (see Appendix Table A4.1 for countrylevel descriptive statistics of income inequality). These coefficients are derived from the high-quality EU-SILC survey covering all EU member states, ensuring a high degree of comparability. For countries not covered by Eurostat, we additionally 
retrieved Gini coefficients from the World Bank (2010) and the OECD (2011). Earlier research demonstrated that the income inequality-health association is largely independent of the choice of the income inequality measure (Judge et al., 1998), and recent research has largely relied on the Gini coefficient (Ellwardt et al., 2014; Layte, 2012; Whelan and Maître, 2013; Wilkinson and Pickett, 2010).

Control variables included in our models are marital status/cohabitation $(0=$ not married or cohabiting with a partner, $1=$ married/cohabiting with a partner), sex $(0=$ male, $1=$ female $)$, age, and education as measured in years. Furthermore, the log of GDP per capita was controlled to adjust for differences in country wealth. Country-level descriptive statistics of GDP per capita are reported in Appendix Table A4.1.

\subsubsection{Method and modeling strategy}

In order to test the hypotheses we derived from the theory, we estimate multilevel (random coefficient) models, with respondents nested in countries (Snijders and Boskers, 2012). Our model includes a random intercept, thus allowing for country-specific constant terms in the regression equations. In order to facilitate interpretation of interactions and the random components, all continuous predictor variables have been grand-mean centered. We have tested a variety of alternative model specifications including random slopes and different shapes of the Gini coefficient (see the Appendix of this Chapter), however, they all lead substantially to the same conclusions.

In the first models (Model 1a and 1b) of Table 4.2, we check for the presence of an inequality-health correlation and an income gradient in health, controlling for various crucial covariates such as GDP per capita, and test Hypotheses 1 and 2. In Models $2 \mathrm{a}$ and $2 \mathrm{~b}$, we add the income comparison orientation variable to the equation, and in Models $3 \mathrm{a}$ and $3 \mathrm{~b}$ we enter the interaction between income inequality and income comparison orientation variable to test Hypotheses 3 and 4 .

\subsection{Results}

\subsubsection{Main findings}

Results of our analyses are presented in Table 4.2. Models 1a and 1b show that there is an income gradient both in psychological wellbeing and in self-rated health: The higher one's income, the better one's psychological wellbeing and the better one's overall health. This confirms Hypothesis 1, which had posited a positive 
relationship between relative income and health. For self-rated health, the gradient is somewhat steeper, as all income groups are significantly worse off than the highest income quintile. For psychological wellbeing, the fourth quintile does not differ significantly from the fifth, thus gains in wellbeing seem to level off more strongly for each additional Euro.

With respect to Hypothesis 2, we find a negative effect of income inequality on both psychological wellbeing and self-rated health, while holding individual income and country wealth constant. This confirms Hypothesis 2, which had suggested that there would be such a negative relationship. For a one-point increase in the Gini coefficient (approximately the difference between the Republic of Ireland and the United Kingdom), psychological wellbeing drops by .007, and self-rated health is reduced by .016. For an increase of the Gini coefficient by one standard deviation (4.7), psychological wellbeing drops by $(S D(X) / S D(Y) \cdot b=4.7 / .46 \cdot .007) .068$ standard deviations, and self-rated health is reduced by $(4.7 / .78 \cdot .016) .10$ standard deviations. Substantially, this effect is small, but this is in line with the findings of earlier research such as Kondo et al. (2009).

Our control variables behave as suggested by earlier research: Women report somewhat lower psychological wellbeing and general health than men, those who are married or cohabiting report being healthier than those who are not married or cohabiting, and health and psychological wellbeing decline with age.

Models $2 \mathrm{a}$ and $2 \mathrm{~b}$ add income comparison orientation to the equation. Income comparison orientation is negatively related to psychological wellbeing and self-rated health, meaning that individuals who find income comparisons more important have worse mental and overall health. When comparing the coefficients of the income quintiles across the respective models, we see that there has been no substantial change in their sizes. This indicates that the income gradient in health is not mediated by income comparison orientation.

Models $3 \mathrm{a}$ and $3 \mathrm{~b}$ then test Hypothesis 3, which had posited that income comparison orientation moderates the income gradient in health in the sense that those who are more prone towards income comparisons suffer more from low income. Both for psychological wellbeing and self-rated health, we can see that none of the interaction terms are significant; thus, we do not find any empirical support for this hypothesis.

Models 4a and 4b test Hypothesis 4, which had stated that the negative health effects of income inequality will be worse for those who are more strongly oriented towards income comparisons. Again, the interaction terms are not significant; thus, we also do not find support for this hypothesis. 


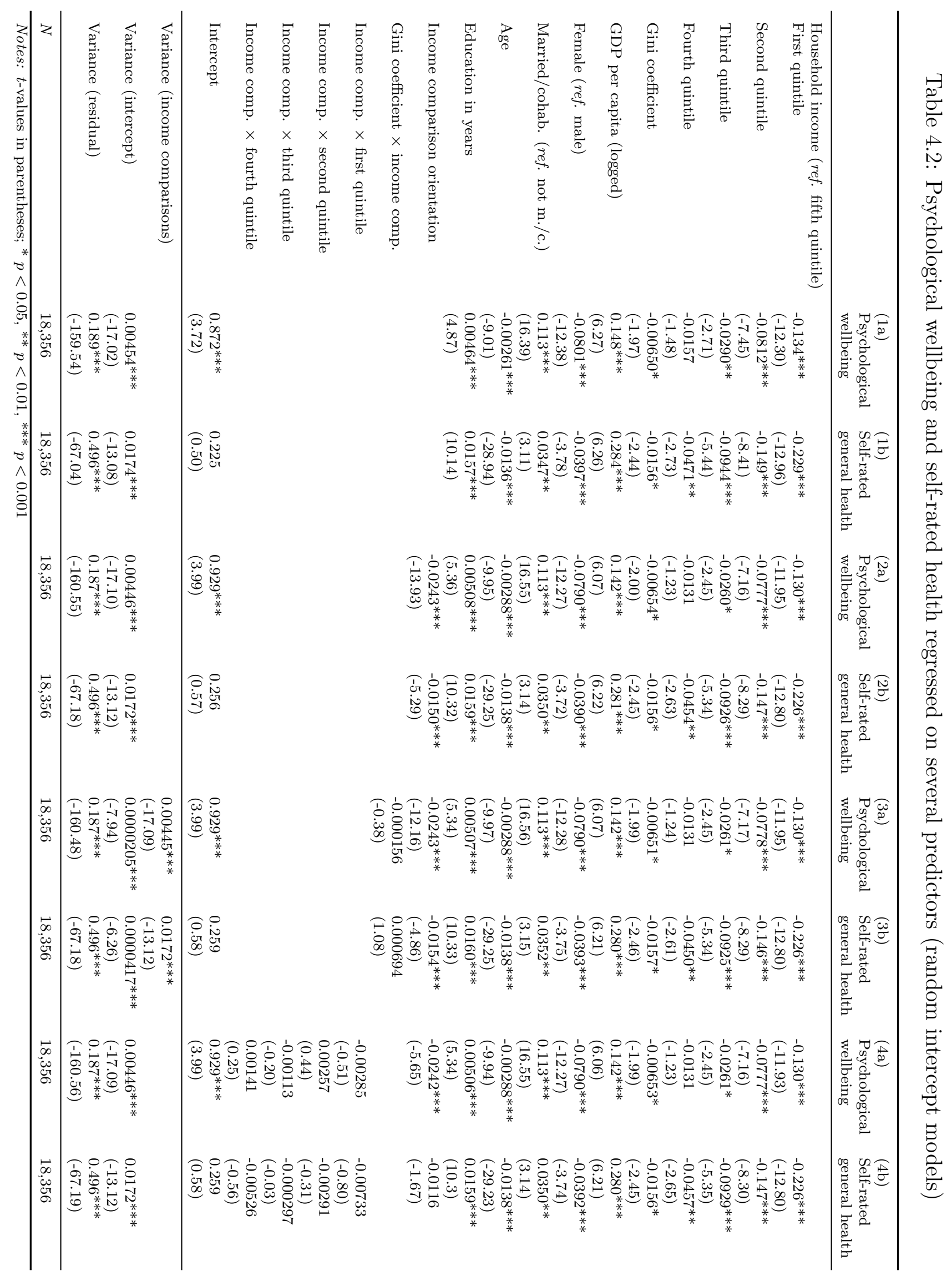




\subsubsection{Sensitivity analyses}

In order to assess the robustness of our findings, we have conducted various sensitivity analyses, which are presented in the Appendix of this Chapter. We show that our findings are by and large robust to different age cut-offs, a more detailed partnership variable, an alternative treatment of the missing data on income, a categorized Gini coefficient, random slopes for all predictors, and averaging the Gini coefficient over several years. Furthermore, we show the results of including different interaction terms.

\subsection{Discussion}

Using a sample of 18,356 Europeans from 23 countries, our study investigated the role of income comparisons in two important contemporary puzzles that medical sociology and social stratification research aim to solve: the socioeconomic gradient in health and the negative income inequality-health correlation. In line with previous theory and evidence, we found an income gradient both in self-rated health and in psychological wellbeing, with the former being somewhat steeper than the latter. Furthermore, we found a negative correlation of income inequality with self-rated health and psychological wellbeing.

Our key finding, however, was that the individual importance attributed to income comparisons did not moderate the effects of relative income or income inequality on health. This finding suggests that those who are most likely to experience stress in income comparisons do not have health outcomes different from those who care less about income comparisons. Despite using two different, yet very important and established health outcomes, and despite using only rather restricted models, we were not able to find a moderating effect of income comparisons.

This finding has important theoretical implications. It casts doubt on the crucial role that researchers such as Wilkinson and Pickett (2010) or Marmot (2004) have attributed to the mechanism of status differentiation that is assumed to link social stratification and health outcomes. One explanation might be that status hierarchies are irrelevant as a causal pathway; instead it is other pathways that are linking socioeconomic status, income inequality, and health. With respect to income inequality, this is in line with the recent findings by Beckfield (2004) and Kondo et al. (2009), who reported that the negative income inequality-health correlation is reduced or disappears when unobserved factors are accounted for. Another explanation of the associations found could be that individuals choose their reference groups wisely, for instance, identify with better-off others (Huguet 
et al., 2009) and do not engage in 'unhealthy' upward income comparisons, a possibility that is consistent with earlier findings of social comparison research (Suls and Wheeler, 2000). Regardless of which it is, our findings suggest that explanations of health inequities or negative effects of inequality that rely on status competition should be called into question.

Also, limitations of the current study need to be mentioned. Although income is an important, if not the most important, marker of social prestige in modern industrialized countries, future research should take other indicators of social status into account (Goldthorpe, 2010). A potential problem in our specific case was that we had only household income available, but our information on income comparison orientation referred to only one household member (in paid work). It would have been ideal to be able to distinguish between household income and incomes of individual household members, but to our knowledge, no large cross-national data sets has both information on health and different types of income. Even if that data were available, it would be difficult to make a clear-cut case for opting for one over the other. Families mostly pool their incomes, with women often being 'secondary' earners, which means that looking only at individual income might grossly misrepresent one's actual resources and social position (cf. for instance the 'dominance principle' of Erikson and Goldthorpe, 1992). Using individual income only as a status marker might have led to biases for groups such as housewives, secondary earners, the unemployed, or pensioners. Furthermore, our sample is restricted to those active on the labor market. Our findings with respect to the effects of income inequality and relative income were in line with those that are seen in general population samples. Despite the fact that the labor force is an important and large part of the population, it might cause some selectivity in our analyses, as those not working are more likely to be severely ill, or income comparisons might only have an effect for those outside of the labor force at the very bottom of the income distribution. This affects the generalizability of our results, warranting further research. Also, our measure of social comparisons was rather limited. More detailed information on social comparisons, such as to whom one is comparing oneself to, and a multi-item measure of social comparison orientation (Schneider and Schupp, 2014) might yield deeper insights into the generating mechanisms of health inequalities.

Notwithstanding these limitations, our paper provides important empirical evidence for two of the most important phenomena at the interface between social stratification research and public health research: the inequality-health association and the SES gradient in health. Our findings suggest that the role of status comparisons for these phenomena might not be as crucial as has often been assumed and that research should focus on alternative pathways for understanding 
these important associations.

\section{Appendix}

This Appendix presents additional descriptive statistics and a variety of sensitivity analyses of the results presented in the main text of the Chapter.

\section{Country-level descriptive statistics}

Appendix Table A4.1 reports country-level descriptive statistics for the Gini coefficient and GDP per capita and gives individual-level sample sizes per country. 
Table A4.1: Descriptive statistics of country-level variables, $N=23$

\begin{tabular}{|c|c|c|c|}
\hline Country & Gini coefficient & GDP per capita & Sample size \\
\hline $\mathrm{AT}$ & 26.2 & 30,700 & 860 \\
\hline $\mathrm{BE}$ & 27.8 & 28,000 & 820 \\
\hline BG & 31.2 & 9,000 & 443 \\
\hline $\mathrm{CH}$ & 28.0 & 35,000 & 913 \\
\hline $\mathrm{DE}$ & 26.8 & 27,500 & 1,156 \\
\hline DK & 23.7 & 30,600 & 856 \\
\hline $\mathrm{EE}$ & 33.4 & 15,600 & 568 \\
\hline ES & 31.2 & 24,700 & 659 \\
\hline $\mathrm{FI}$ & 25.9 & 27,000 & 951 \\
\hline $\mathrm{FR}$ & 27.3 & 25,700 & 984 \\
\hline GB & 32.5 & 28,500 & 1,080 \\
\hline $\mathrm{HU}$ & 33.3 & 14,900 & 518 \\
\hline IE & 31.3 & 34,400 & 663 \\
\hline NL & 26.4 & 31,000 & 983 \\
\hline $\mathrm{NO}$ & 30.0 & 43,400 & 1,181 \\
\hline PL & 33.3 & 12,300 & 655 \\
\hline $\mathrm{PT}$ & 36.8 & 19,600 & 555 \\
\hline $\mathrm{RO}$ & 33.0 & 9,100 & 686 \\
\hline $\mathrm{RU}$ & 44.0 & 6,947 & 967 \\
\hline $\mathrm{SE}$ & 24.0 & 29,100 & 1,191 \\
\hline SI & 23.7 & 20,700 & 554 \\
\hline SK & 24.5 & 17,000 & 572 \\
\hline UA & 28.0 & 2,303 & 541 \\
\hline Average & 29.7 & 22,741 & 798 \\
\hline$S D$ & 4.7 & 10,382 & - \\
\hline
\end{tabular}

Note: Gini coefficient and GDP per capita taken from

Eurostat (2011), OECD (2011), and World Bank (2010)

\section{Sensitivity analyses}

In order to assess the robustness of our findings, we present a number of sensitivity analyses in this Appendix. 


\section{Different age limits}

Firstly, we present models with different upper age limits. In the Chapter, we are presenting models with an upper age limit of age 70, however it is known that countries differ substantially in mortality after age 60, potentially leading to bias due to a select sample. Thus, in Table A4.2, we present a model where we do not impose any upper age limit, in Table A4.3, we exclude respondents older than 65, and in Table A4.4, we exclude respondents age older than 60. All age limits yield substantially similar results to those presented in the paper. 


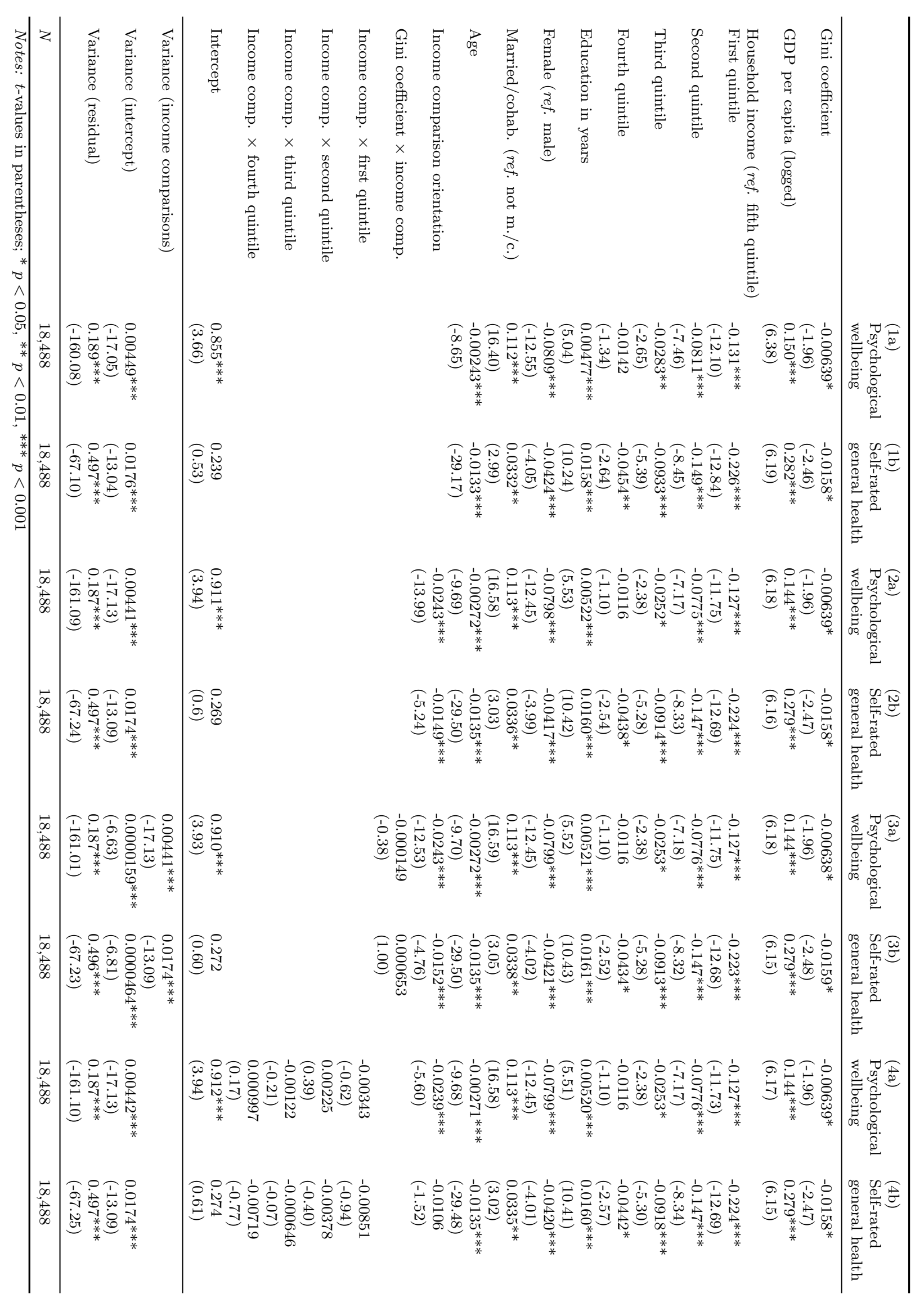

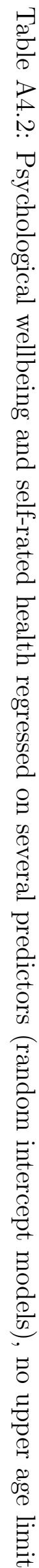




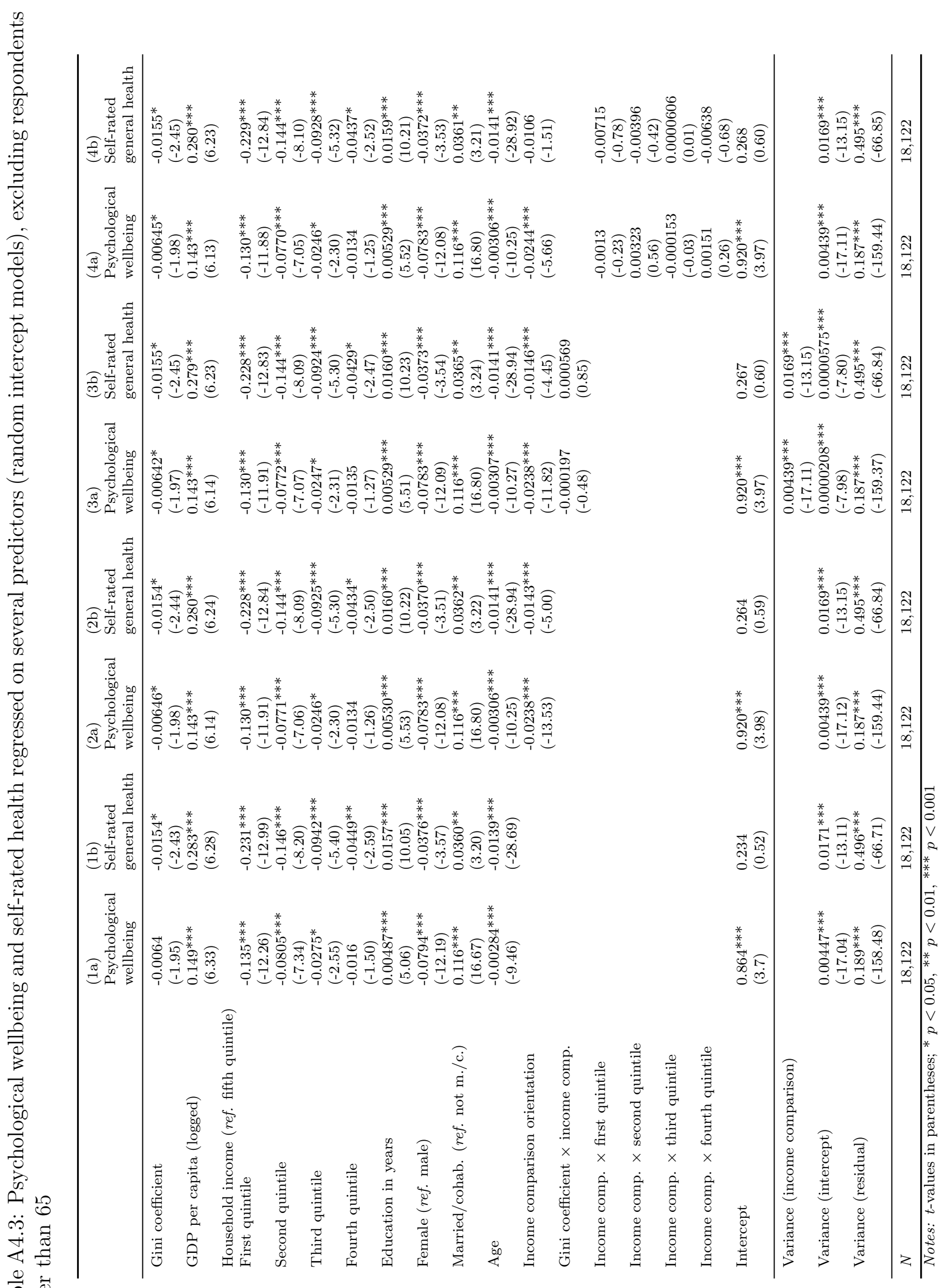




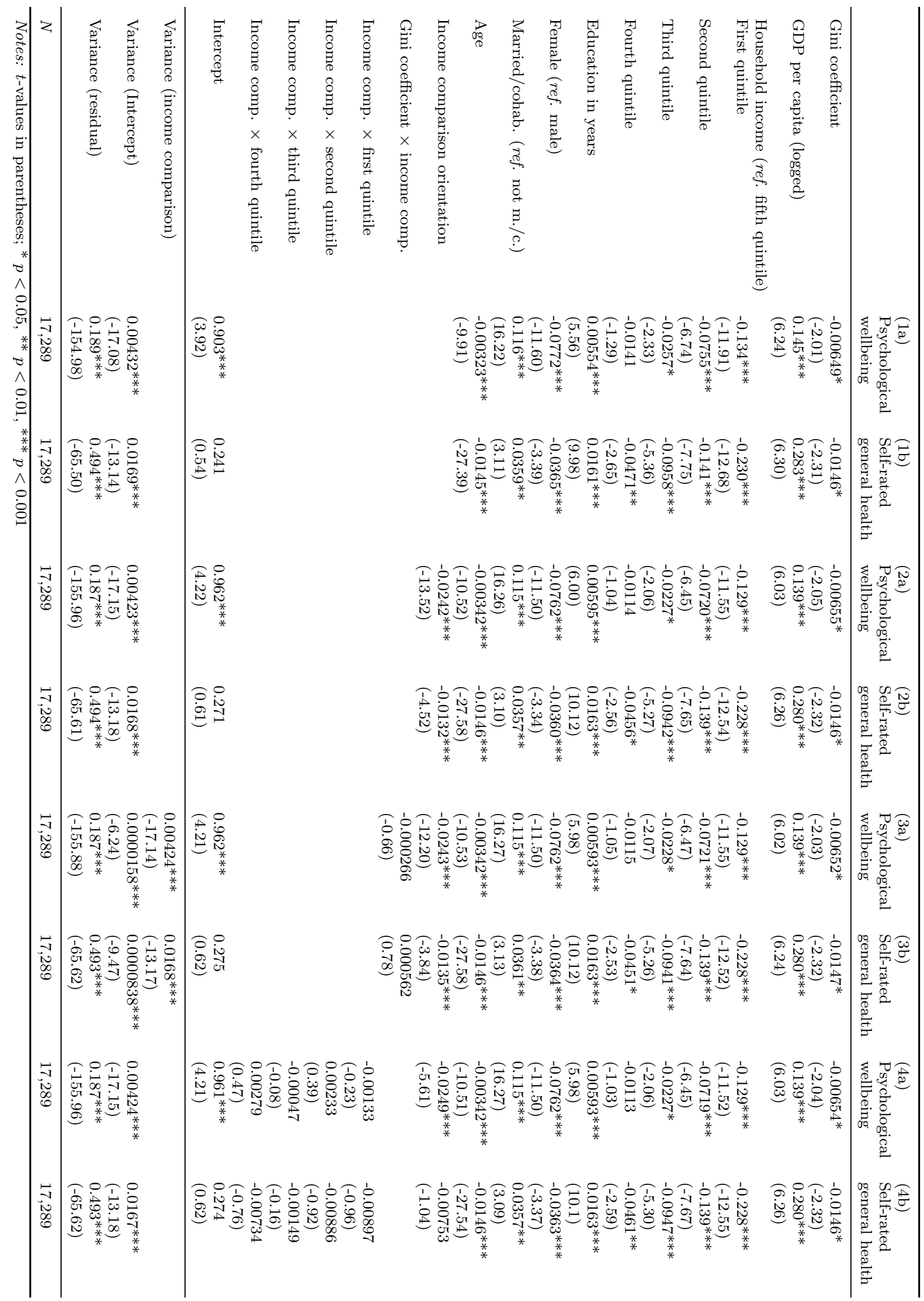




\section{Expanded partnership variable}

Secondly, we present models with an expanded partnership variable (Table A4.5). For the sake of parsimony, we distinguished only between respondents who are married and/or cohabiting with a partner versus those who do not in our paper. However, since it is known that marital status is an important predictor of self-rated health (Huijts et al., 2010b) and Clark and Senik (2010) have shown important differences between singles and married respondents in their income comparison orientation, we present a fuller picture of the relationships between marital status and health in this Appendix.

Therefore, in Table A4.5, we present models distinguishing between (1) married respondents, respondents cohabiting with a partner, as well as respondents living with a civil partnership (CP) agreement, (2) divorced respondents, separated respondents, or respondents who have dissolved a CP, (3) widows and widowers as well as respondents who were in a CP where the partner has died, and (4) singles. 


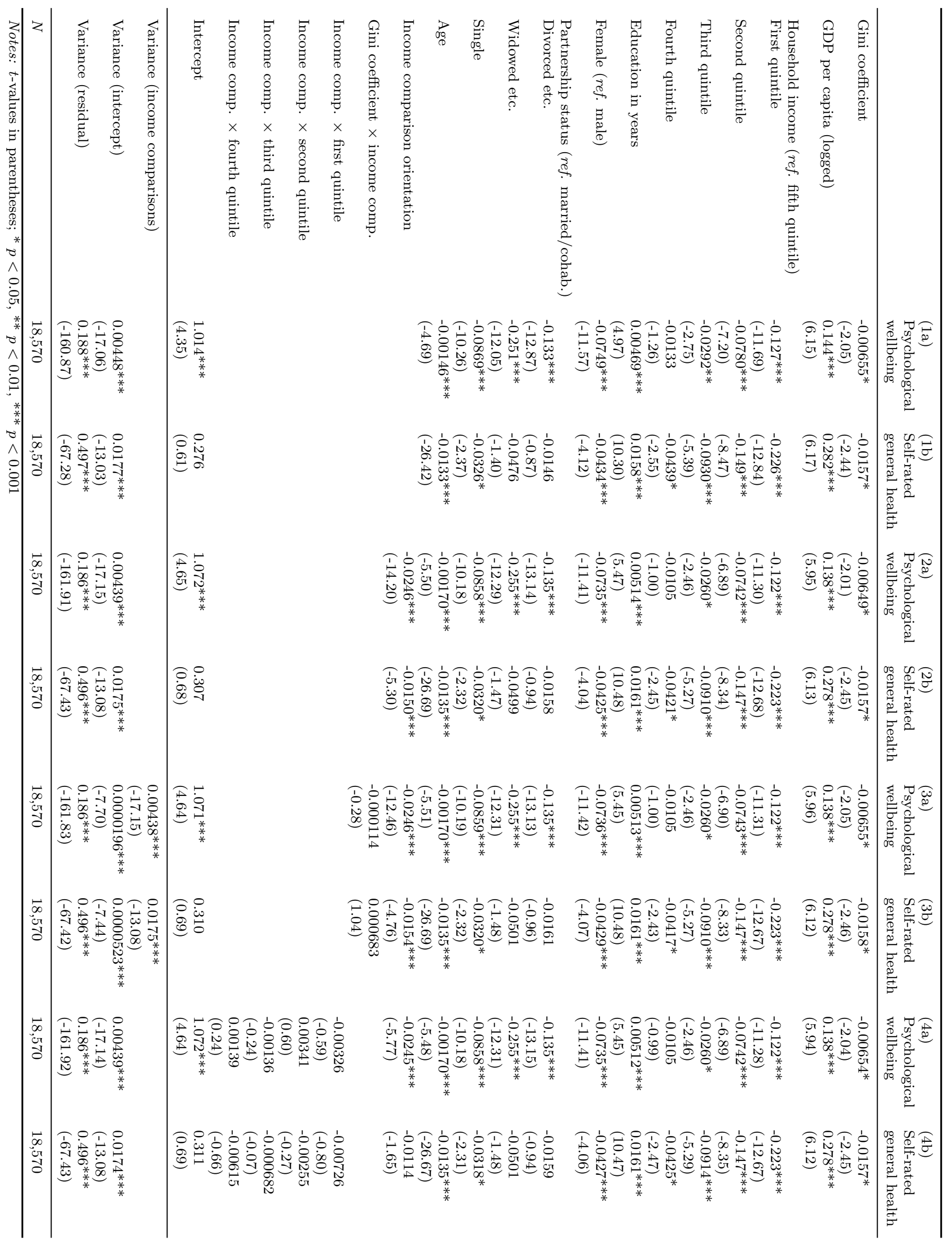

足

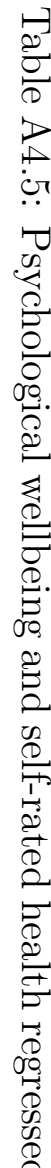

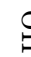

送 
As can be seen in Table A4.5, for psychological wellbeing there are statistically significant differences between the married and cohabiting group versus all other groups. These differences all point in the same direction: Married and cohabiting individuals enjoy greater psychological wellbeing than those who are divorced, widowed, or single. For self-rated health, a similar picture arises, but not all differences are statistically significant. The general picture is that married and cohabiting individuals report better health than all other groups; however, this difference is only significant for single individuals.

All points of substantive interest remain substantially the same for all models, regardless of what version of the variable is used.

\section{Missing income information}

Thirdly, we inspect the impact of dealing with missing values in our data. After removing respondents not active on the labor market, around 19 per cent of cases in the sample fail to report an income. In the analyses presented in the paper, we have removed those respondents from the sample. As a sensitivity check of our findings, we have included these cases using an additional dummy variable that denotes those cases who did not report an income. This dummy variable approach is a traditional technique to deal with missing data and is still often being used nowadays; for example, Layte (2012) uses the same approach in his paper on income inequality and mental health.

Our results using this approach are presented in Table A4.6. Results show that those failing to report an income differ somewhat from the highest income quintile, but less than those in the first and second quintile, suggesting that those who did not report an income likely draw from diverse income groups. In terms of substantive results, there is no change when using this approach. 


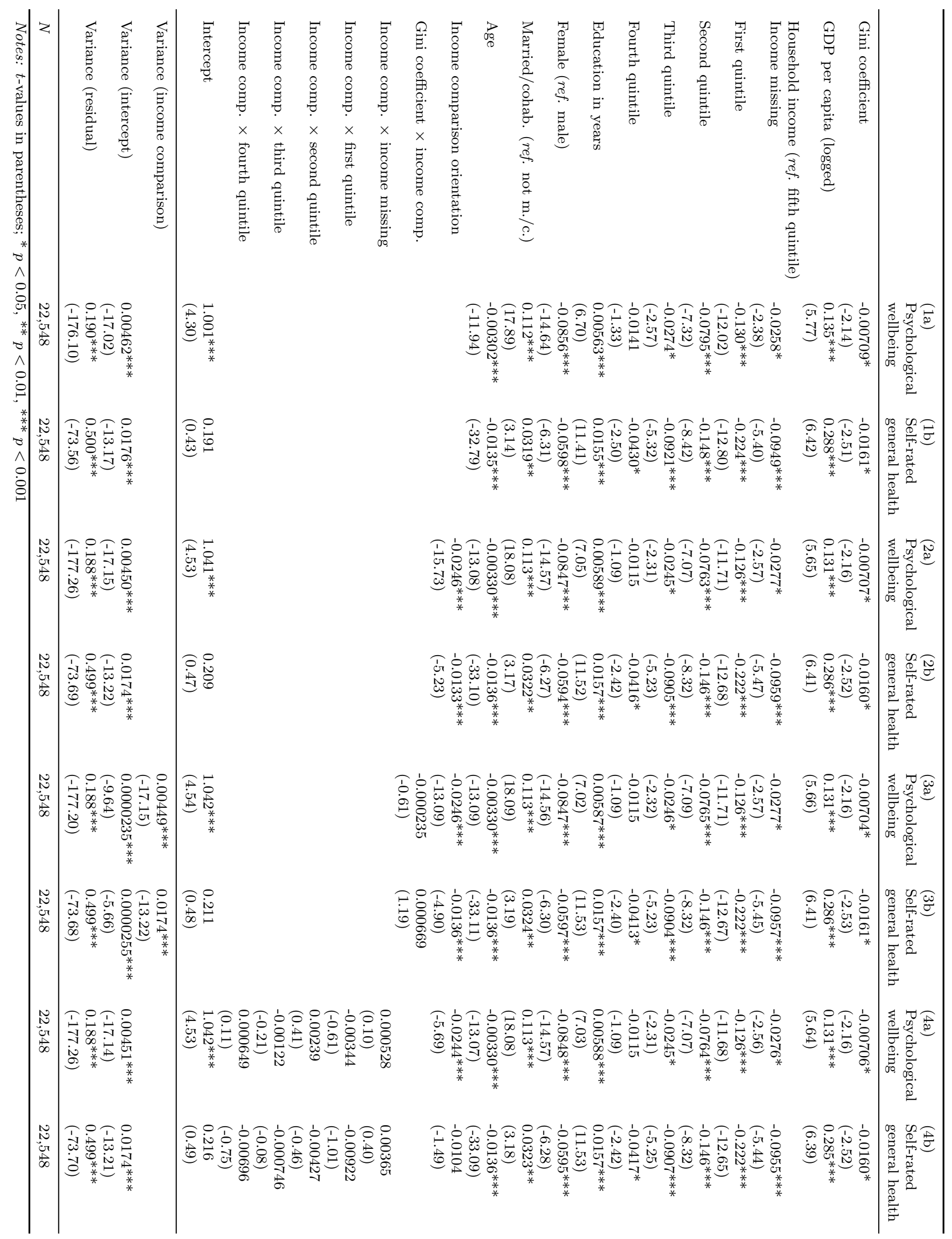




\section{Categorical Gini coefficient}

Fourthly, we present analyses where we have collapsed countries into three categories based on their level of income inequality. This allows for seeing whether the slope of income comparison orientation changes at the extremes of the Gini coefficient.

We have tested such an approach and found little difference in the substantive results (see Table A4.7). In these models, we categorized the Gini coefficient into three groups, namely

- low $\left(\leq 33^{\text {rd }}\right.$ percentile $)$,

- $\operatorname{medium}\left(>33^{\text {rd }}\right.$ percentile and $\leq 67^{\text {th }}$ percentile), and

- $\operatorname{high}\left(>67^{\text {th }}\right.$ percentile).

For self-rated health, it shows that the difference between the medium and the low group is not statistically significant; however, it points into the same direction. For all substantial purposes, results remained the same. However, convergence was greatly hampered for some of the models. 


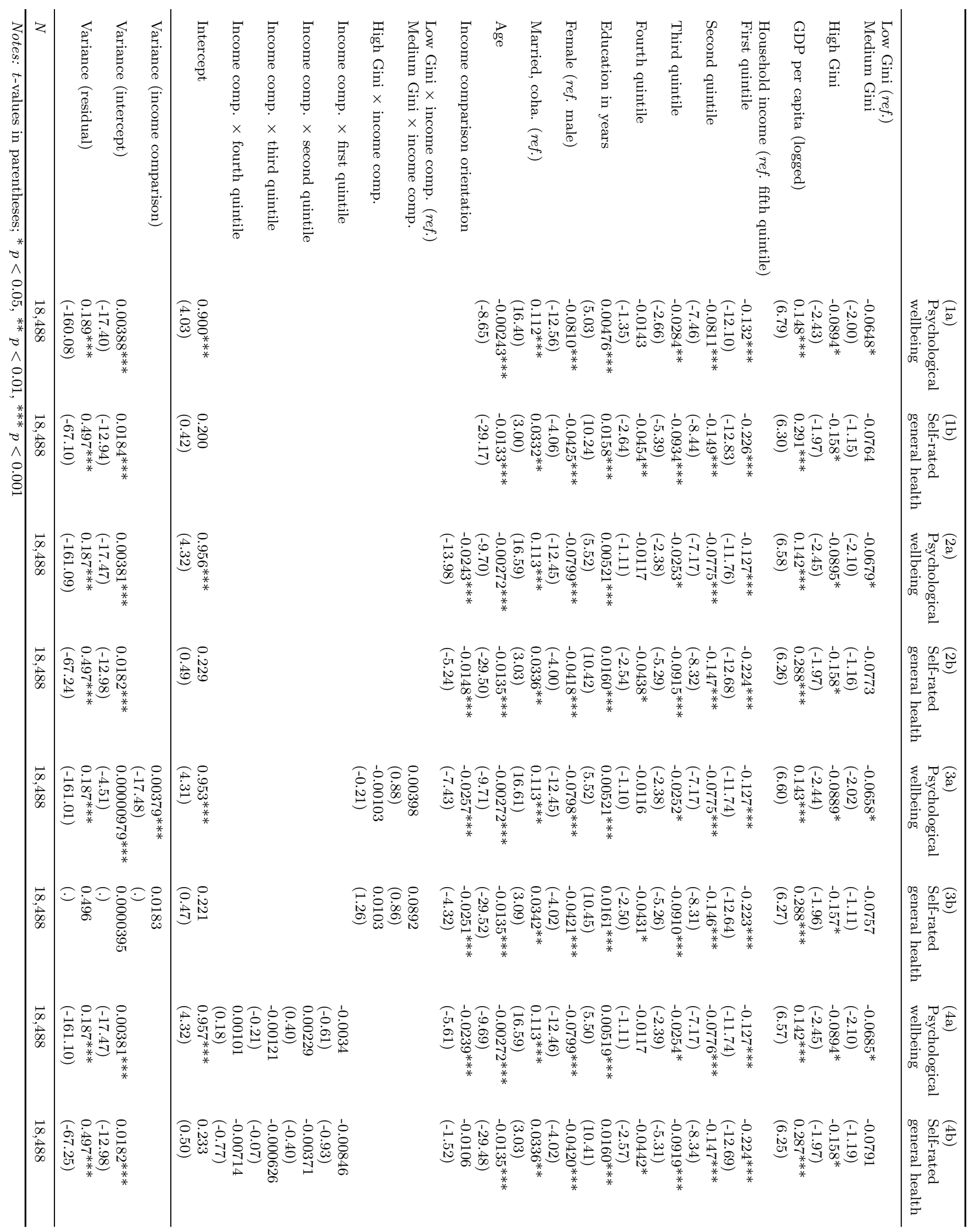

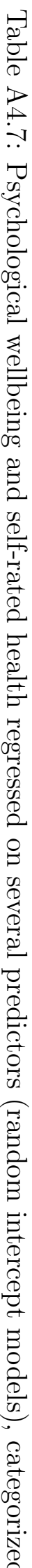

: 


\section{Random slopes}

Fifthly, we present models where we allow the slopes of the individual-level predictors to vary across countries. In Table A4.8, selected models with random slopes for education, gender, marital status, and age are reported. Comparing the Deviances as reported at the bottom of the Table shows that they all improve model fit, yet they hardly affect the substantive findings. In order to present simpler and more parsimonious models, we decided to present a random intercept model in the main text and only mention the robustness of our findings, even when including random slopes. 


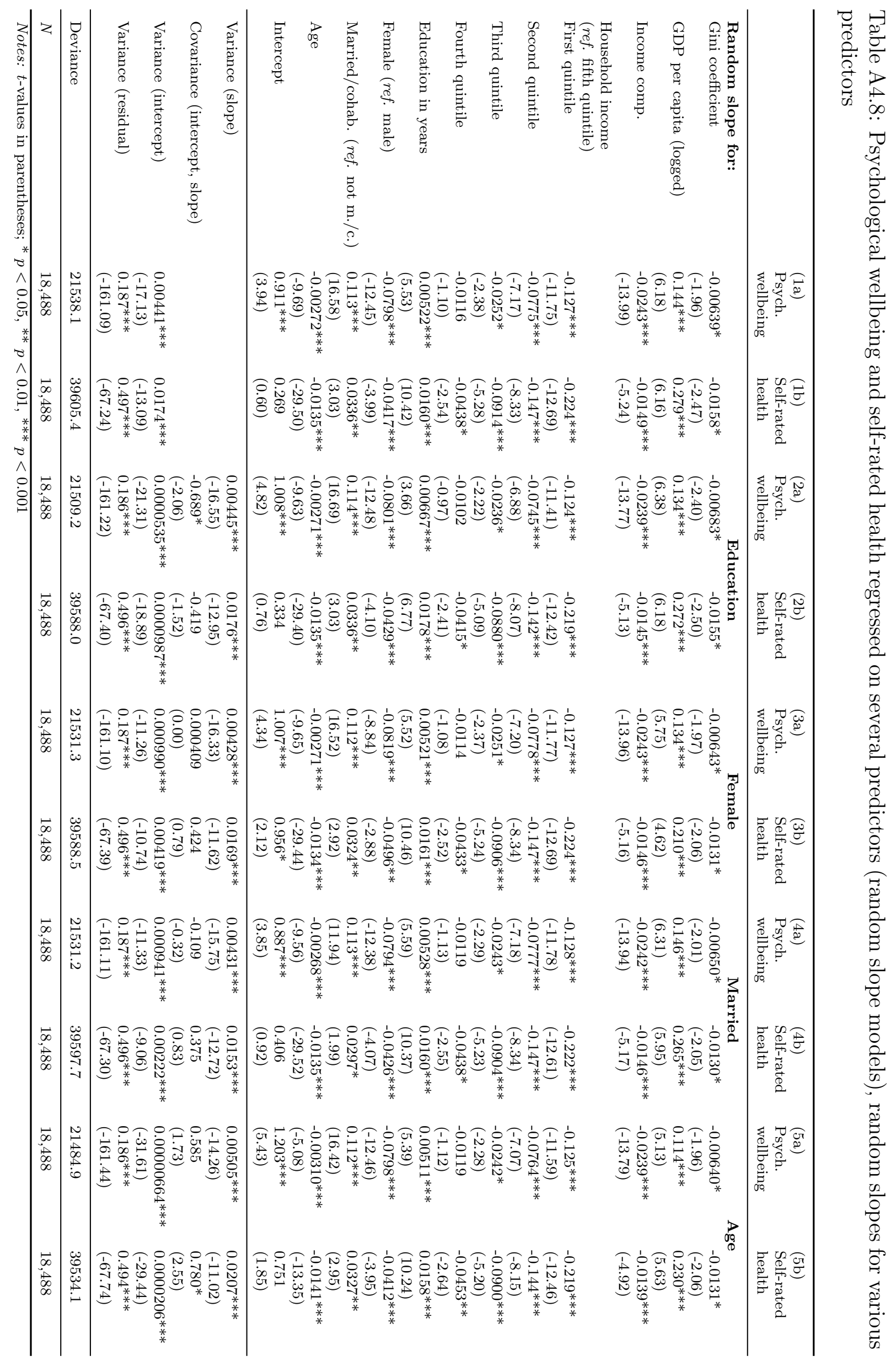


Fitting a random slope model for income in the shape of quintile dummies turned out to be impossible as it would require freeing four slopes in the model. Given the limited number of level-2 units (23 countries), such a model cannot converge. Instead, we have included a linear term for income quintiles (ranging from 1 - poorest quintile to 5-richest quintile) and have allowed the slope of that linear term to vary. Selected models with a random income quintile slope are reported in Table A4.9. Again, the substantial findings remain the same.

We are aware of the possibility of using a two-step multilevel procedure to circumvent the problem of fitting a random slope model with several random slopes for the income dummies (e.g. Wooldridge, 2010). However, this approach then creates the problem how to fit a model with several outcome variables (the income dummy coefficients) at the same time due to the computational problems and the restrictive assumptions that come with such models (Tabachnick and Fidell, 2007), therefore we have abstained from this approach.

\section{Income inequality averaged over several years}

Sixthly, we assessed whether our findings are robust against year-to-year volatility in the Gini coefficient. As income inequality can vary over the course over several years, for instance together with the business cycle (Atkinson, 2003), we have averaged Gini coefficients over several years (2004 to 2008) for those countries where yearly data was available.

These models are presented in Table A4.10. Results remain substantially the same, which is not surprising as the correlation between the single-year and average Gini coefficients is high, Pearson's $r=.95$. 
Table A4.9: Psychological wellbeing and self-rated health regressed on several predictors (random slope models), random slope for income quintiles

\begin{tabular}{|c|c|c|c|c|}
\hline & $\begin{array}{l}(1 \mathrm{a}) \\
\text { Psychological } \\
\text { wellbeing }\end{array}$ & $\begin{array}{l}(1 \mathrm{~b}) \\
\text { Self-rated } \\
\text { general health }\end{array}$ & $\begin{array}{l}(2 \mathrm{a}) \\
\text { Psychological } \\
\text { wellbeing }\end{array}$ & $\begin{array}{l}(2 \mathrm{~b}) \\
\text { Self-rated } \\
\text { general health }\end{array}$ \\
\hline Gini coefficient & $\begin{array}{l}-0.00639 * \\
(-1.96)\end{array}$ & $\begin{array}{l}-0.00638 \\
(-1.95)\end{array}$ & $\begin{array}{l}-0.0158^{*} \\
(-2.47)\end{array}$ & $\begin{array}{l}-0.0154^{*} \\
(-2.47)\end{array}$ \\
\hline GDP per capita (logged) & $\begin{array}{l}0.143^{* * *} \\
(6.10)\end{array}$ & $\begin{array}{l}0.154^{* * *} \\
(7.98)\end{array}$ & $\begin{array}{l}0.279^{* * *} \\
(6.16)\end{array}$ & $\begin{array}{l}0.277^{* * *} \\
(6.23)\end{array}$ \\
\hline Income comparison orientation & $\begin{array}{l}-0.0243^{* * *} \\
(-13.94)\end{array}$ & $\begin{array}{l}-0.0243^{* * *} \\
(-13.95)\end{array}$ & $\begin{array}{l}-0.0148^{* * *} \\
(-5.23)\end{array}$ & $\begin{array}{l}-0.0146^{* * *} \\
(-5.17)\end{array}$ \\
\hline Income quintile & $\begin{array}{l}0.0329^{* * *} \\
(13.70)\end{array}$ & $\begin{array}{l}0.0336^{* * *} \\
(10.31)\end{array}$ & $\begin{array}{l}0.0556^{* * *} \\
(14.21)\end{array}$ & $\begin{array}{l}0.0559^{* * *} \\
(11.17)\end{array}$ \\
\hline Education & $\begin{array}{l}0.00514^{* * *} \\
(5.44)\end{array}$ & $\begin{array}{l}0.00504^{* * *} \\
(5.34)\end{array}$ & $\begin{array}{l}0.0160^{* * *} \\
(10.40)\end{array}$ & $\begin{array}{l}0.0161^{* * *} \\
(10.43)\end{array}$ \\
\hline Female (ref. male) & $\begin{array}{l}-0.0794^{* * *} \\
(-12.38)\end{array}$ & $\begin{array}{l}-0.0791^{* * *} \\
(-12.34)\end{array}$ & $\begin{array}{l}-0.0416^{* * *} \\
(-3.98)\end{array}$ & $\begin{array}{l}-0.0413^{* * *} \\
(-3.96)\end{array}$ \\
\hline Married/cohab. (ref. not m./c.) & $\begin{array}{l}0.114^{* * *} \\
(16.75)\end{array}$ & $\begin{array}{l}0.114^{* * *} \\
(16.81)\end{array}$ & $\begin{array}{l}0.0343^{* *} \\
(3.10)\end{array}$ & $\begin{array}{l}0.0347^{* *} \\
(3.13)\end{array}$ \\
\hline Age & $\begin{array}{l}-0.00271^{* * *} \\
(-9.67)\end{array}$ & $\begin{array}{l}-0.00270^{* * *} \\
(-9.62)\end{array}$ & $\begin{array}{l}-0.0135^{* * *} \\
(-29.49)\end{array}$ & $\begin{array}{l}-0.0135^{* * *} \\
(-29.43)\end{array}$ \\
\hline Intercept & $\begin{array}{l}0.769^{* * * *} \\
(3.29)\end{array}$ & $\begin{array}{l}0.664^{* * *} \\
(3.46)\end{array}$ & $\begin{array}{l}0.000775 \\
(0.00)\end{array}$ & $\begin{array}{l}0.0229 \\
(0.05) \\
\end{array}$ \\
\hline Variance (intercept) & $\begin{array}{l}0.00450^{* * *} \\
(-17.08)\end{array}$ & $\begin{array}{l}0.000109^{* * *} \\
(-14.17)\end{array}$ & $\begin{array}{l}0.0174^{* * * *} \\
(-13.08)\end{array}$ & $\begin{array}{l}0.000215^{* * *} \\
(-11.47)\end{array}$ \\
\hline Variance (residual) & $\begin{array}{l}0.187 * * * \\
(-160.99)\end{array}$ & $\begin{array}{l}0.187 * * * \\
(-160.98)\end{array}$ & $\begin{array}{l}0.497 * * * \\
(-67.22)\end{array}$ & $\begin{array}{l}0.496^{* * *} \\
(-67.26)\end{array}$ \\
\hline Variance (slope) & & $\begin{array}{l}0.00942^{* * *} \\
(-13.42)\end{array}$ & & $\begin{array}{l}0.0233^{* * *} \\
(-10.99)\end{array}$ \\
\hline Covariance (intercept, slope) & & $\begin{array}{l}-2.185 \\
(-1.22)\end{array}$ & & $\begin{array}{l}-0.681 \\
(-1.92) \\
\end{array}$ \\
\hline Deviance & 21558.0 & 21543.1 & 39608.6 & 39603.7 \\
\hline$N$ & 18,488 & 18,488 & 18,488 & 18,488 \\
\hline
\end{tabular}




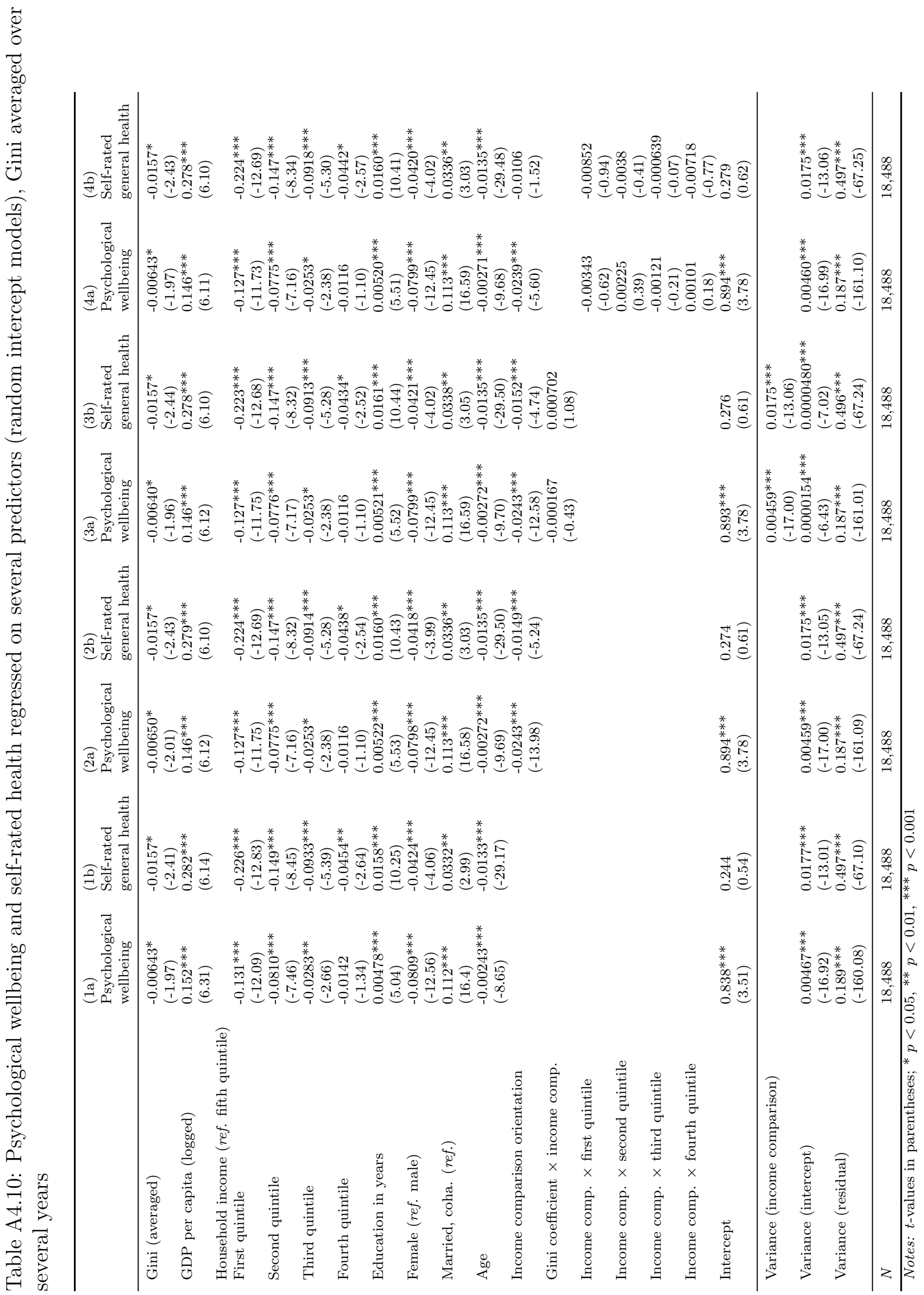


Three-way interaction between income, income comparison orientation, and income inequality

Finally, a common criticism of Wilkinson and Pickett's (2010) work pertains to their claim that income inequality negatively affects everyone regardless of their income rather than predominately those at the bottom of the income distribution (Lancee and Van de Werfhorst, 2012; Neckermann and Torche, 2007; Runciman, 2009). Critics make a case that is those at the bottom of the income distribution who suffer from the effects of income inequality, whereas those at the top are presumably largely unaffected. In order to test the tenability of such claims, we ran a number of additional models with interaction terms, displayed in Table A4.11.

Interaction terms between income quintiles and income inequality remained non-significant in our models (1a and 1b) - thus supporting the claim of Wilkinson and Pickett (2010). Those at the bottom at the income distribution appear to not suffer more from income inequality than those at the top.

Models $2 \mathrm{a}$ and $2 \mathrm{~b}$ of Table A4.11 show a three-way interaction between income quintiles, income comparison orientation, and income inequality. Again, no significant differences can be found. 
Table A4.11: Psychological wellbeing and self-rated health regressed on several predictors (random intercept models), three-way interaction

\begin{tabular}{|c|c|c|c|c|}
\hline & $\begin{array}{l}(1 \mathrm{a}) \\
\text { Psychological } \\
\text { wellbeing }\end{array}$ & $\begin{array}{l}\text { (1b) } \\
\text { Self-rated } \\
\text { general health }\end{array}$ & $\begin{array}{l}(2 \mathrm{a}) \\
\text { Psychological } \\
\text { wellbeing }\end{array}$ & $\begin{array}{l}\text { (2b) } \\
\text { Self-rated } \\
\text { general health }\end{array}$ \\
\hline Gini coefficient & $\begin{array}{l}-0.00522 \\
(-1.47)\end{array}$ & $\begin{array}{l}-0.0128 \\
(-1.87)\end{array}$ & $\begin{array}{l}-0.00521 \\
(-1.46)\end{array}$ & $\begin{array}{l}-0.0128 \\
(-1.88)\end{array}$ \\
\hline GDP per capita (logged) & $\begin{array}{l}0.144^{* * *} \\
(6.19)\end{array}$ & $\begin{array}{l}0.279^{* * *} \\
(6.14)\end{array}$ & $\begin{array}{l}0.144^{\text {*** }} \\
(6.20)\end{array}$ & $\begin{array}{l}0.278^{* * *} \\
(6.14)\end{array}$ \\
\hline Household income (ref. fifth quintile) & & & & \\
\hline First quintile & $\begin{array}{l}-0.127^{* * *} \\
(-11.71)\end{array}$ & $\begin{array}{l}-0.223^{* * *} \\
(-12.62)\end{array}$ & $\begin{array}{l}-0.128^{* * *} \\
(-11.80)\end{array}$ & $\begin{array}{l}-0.223^{* * *} \\
(-12.64)\end{array}$ \\
\hline Second quintile & $\begin{array}{l}-0.0785^{* * *} \\
(-7.24)\end{array}$ & $\begin{array}{l}-0.147^{* * *} \\
(-8.31)\end{array}$ & $\begin{array}{l}-0.0787^{* * *} \\
(-7.25)\end{array}$ & $\begin{array}{l}-0.147^{* * *} \\
(-8.32)\end{array}$ \\
\hline Third quintile & $\begin{array}{l}-0.0261^{*} \\
(-2.45)\end{array}$ & $\begin{array}{l}-0.0905^{* * *} \\
(-5.21)\end{array}$ & $\begin{array}{l}-0.0264^{*} \\
(-2.48)\end{array}$ & $\begin{array}{l}-0.0912^{* * *} \\
(-5.25)\end{array}$ \\
\hline Fourth quintile & $\begin{array}{l}-0.0112 \\
(-1.06)\end{array}$ & $\begin{array}{l}-0.0433^{*} \\
(-2.51)\end{array}$ & $\begin{array}{l}-0.0113 \\
(-1.07)\end{array}$ & $\begin{array}{l}-0.0439 * \\
(-2.55)\end{array}$ \\
\hline Income comp. (ICO) & $\begin{array}{l}-0.0243^{* * *} \\
(-13.97)\end{array}$ & $\begin{array}{l}-0.0149^{* * *} \\
(-5.25)\end{array}$ & $\begin{array}{l}-0.0236 * * * \\
(-5.50)\end{array}$ & $\begin{array}{l}-0.0106 \\
(-1.51)\end{array}$ \\
\hline Gini $\times$ first quintile & $\begin{array}{l}-0.00213 \\
(-1.00)\end{array}$ & $\begin{array}{l}-0.00261 \\
(-0.75)\end{array}$ & $\begin{array}{l}-0.00252 \\
(-1.17)\end{array}$ & $\begin{array}{l}-0.00267 \\
(-0.76)\end{array}$ \\
\hline Gini $\times$ second quintile & $\begin{array}{l}-0.00416 \\
(-1.83)\end{array}$ & $\begin{array}{l}-0.00523 \\
(-1.41)\end{array}$ & $\begin{array}{l}-0.00417 \\
(-1.84)\end{array}$ & $\begin{array}{l}-0.0052 \\
(-1.40)\end{array}$ \\
\hline Gini $\times$ third quintile & $\begin{array}{l}0.000998 \\
(0.48)\end{array}$ & $\begin{array}{l}-0.00296 \\
(-0.87)\end{array}$ & $\begin{array}{l}0.000993 \\
(0.47)\end{array}$ & $\begin{array}{l}-0.00315 \\
(-0.92)\end{array}$ \\
\hline Gini $\times$ fourth quintile & $\begin{array}{l}-0.000955 \\
(-0.44)\end{array}$ & $\begin{array}{l}-0.00439 \\
(-1.24)\end{array}$ & $\begin{array}{l}-0.000896 \\
(-0.41)\end{array}$ & $\begin{array}{l}-0.00446 \\
(-1.26)\end{array}$ \\
\hline First quintile $\times$ ICO & & & $\begin{array}{l}-0.004 \\
(-0.71)\end{array}$ & $\begin{array}{l}-0.00882 \\
(-0.97)\end{array}$ \\
\hline Second quintile $\times$ ICO & & & $\begin{array}{l}0.00189 \\
(0.33)\end{array}$ & $\begin{array}{l}-0.00373 \\
(-0.40)\end{array}$ \\
\hline Third quintile $\times$ ICO & & & $\begin{array}{l}-0.00201 \\
(-0.35)\end{array}$ & $\begin{array}{l}-0.00173 \\
(-0.18)\end{array}$ \\
\hline Fourth quintile $\times$ ICO & & & $\begin{array}{l}0.000477 \\
(0.08)\end{array}$ & $\begin{array}{l}-0.00681 \\
(-0.73)\end{array}$ \\
\hline $\mathrm{ICO} \times$ Gini & & & $\begin{array}{l}-0.000913 \\
(-1.04)\end{array}$ & $\begin{array}{l}-0.000557 \\
(-0.39)\end{array}$ \\
\hline First quintile $\times$ ICO $\times$ Gini & & & $\begin{array}{l}0.00242 \\
(1.82)\end{array}$ & $\begin{array}{l}0.00128 \\
(0.68)\end{array}$ \\
\hline Second quintile $\times$ ICO $\times$ Gini & & & $\begin{array}{l}-0.000405 \\
(-0.33)\end{array}$ & $\begin{array}{l}0.000397 \\
(0.20)\end{array}$ \\
\hline Third quintile $\times$ ICO $\times$ Gini & & & $\begin{array}{l}0.00113 \\
(1.00)\end{array}$ & $\begin{array}{l}0.0016 \\
(0.87)\end{array}$ \\
\hline Fourth quintile $\times$ ICO $\times$ Gini & & & $\begin{array}{l}0.00013 \\
(0.11)\end{array}$ & $\begin{array}{l}0.00186 \\
(0.97)\end{array}$ \\
\hline Education in years & $\begin{array}{l}0.00521^{* * *} \\
(5.52)\end{array}$ & $\begin{array}{l}0.0160^{* * * *} \\
(10.43)\end{array}$ & $\begin{array}{l}0.00517^{* * *} \\
(5.48)\end{array}$ & $\begin{array}{l}0.0161^{* * *} \\
(10.43)\end{array}$ \\
\hline Female (ref. male) & $\begin{array}{l}-0.0796^{* * *} \\
(-12.42)\end{array}$ & $\begin{array}{l}-0.0417^{* * *} \\
(-3.99)\end{array}$ & $\begin{array}{l}-0.0799^{* * *} \\
(-12.45)\end{array}$ & $\begin{array}{l}-0.0421^{* * * *} \\
(-4.02)\end{array}$ \\
\hline Married/cohab. (ref. not m./c.) & $\begin{array}{l}0.112^{* * *} \\
(16.49)\end{array}$ & $\begin{array}{l}0.0332^{* *} \\
(3.00)\end{array}$ & $\begin{array}{l}0.112^{* * *} \\
(16.52)\end{array}$ & $\begin{array}{l}0.0333^{* *} \\
(3.00)\end{array}$ \\
\hline Age & $\begin{array}{l}-0.00268^{* * *} \\
(-9.55)\end{array}$ & $\begin{array}{l}-0.0135^{* * *} \\
(-29.39)\end{array}$ & $\begin{array}{l}-0.00268^{* * *} \\
(-9.54)\end{array}$ & $\begin{array}{l}-0.0135^{* * *} \\
(-29.39)\end{array}$ \\
\hline Intercept & $\begin{array}{l}0.910^{* * *} \\
(3.94)\end{array}$ & $\begin{array}{l}0.273 \\
(0.61)\end{array}$ & $\begin{array}{l}0.909^{* * *} \\
(3.93)\end{array}$ & $\begin{array}{l}0.278 \\
(0.62)\end{array}$ \\
\hline Variance (intercept) & $\begin{array}{l}0.00440^{* * *} \\
(-17.13)\end{array}$ & $\begin{array}{l}0.0174^{* * *} \\
(-13.08)\end{array}$ & $\begin{array}{l}0.00439^{* * *} \\
(-17.13)\end{array}$ & $\begin{array}{l}0.0174^{* * *} \\
(-13.08)\end{array}$ \\
\hline Variance (residual) & $\begin{array}{l}0.187^{* * *} \\
(-161.13)\end{array}$ & $\begin{array}{l}0.497^{* * *} \\
(-67.25)\end{array}$ & $\begin{array}{l}0.187 * * * \\
(-161.18)\end{array}$ & $\begin{array}{l}0.497 * * * \\
(-67.27)\end{array}$ \\
\hline$N$ & 18,488 & 18,488 & 18,488 & 18,488 \\
\hline
\end{tabular}





\section{Chapter 5}

\section{The Educational Gradient in Self-Rated Health in Europe. Does the Doctor-Patient Relationship Make a Difference? ${ }^{1}$}

\subsection{Introduction}

Social inequality in health and disease is a pervasive phenomenon, both over time and across societies. The mechanisms underlying it, however, are not fully understood (Elo, 2009). Earlier research has suggested that one important cause of social inequality in health is differential access to and quality of health care (Beckfield et al., 2013; Paterson and Judge, 2002). Focusing on a crucial aspect of quality of health care, namely the doctor-patient relationship, this paper studies the effects of different models of doctor-patient interactions on educational inequalities in self-reported health.

A positive relationship between socioeconomic status and health has been documented for individuals both in contemporary societies (Elo, 2009; Mackenbach et al., 2008; Präg et al., 2014), as well as in earlier periods of history. Despite this long tradition of research on health disparities between social strata, little conclusive evidence on the underlying reasons of the social gradient has been produced. The problem lies in understanding the link between socioeconomic status (SES) and health: Why do those with higher socioeconomic status enjoy better health?

\footnotetext{
${ }^{1}$ This chapter is co-authored by Rafael Wittek and Melinda Mills. Earlier versions of this chapter were presented at the Third Dutch European Social Survey Workshop 2010 in The Hague and the Joint ECSR, QMSS2, and Transeurope Conference 2010 in Bamberg. The authors thank the discussants at these occasions for their comments.
} 
Traditional explanations that focus material living conditions and lifestyle factors are seen as incomplete (Elo, 2009). Even though material living conditions have greatly improved in the past century, the educational differential in health has remained intact. Lifestyle factors, such as smoking, drinking, insufficient exercise, and poor diet, also display social gradients, but are substantially smaller than the SES-health gradient (Lantz et al., 2001). The problem is even more puzzling due to the ubiquity of the social gradient: Neither Nordic-style welfare states granting universal access to high-quality health care nor the virtual elimination of infectious diseases as the most prevalent causes of death were able to eradicate the social gradient in health. Furthermore, different aspects of socioeconomic status, such as education, income, and occupational prestige, all tend to show effects on health, even independent of another (Elo, 2009).

Education is a crucial indicator of SES and a particularly important risk factor for poor health (Gesthuizen et al., 2012; Mirowsky and Ross, 2003). The lower educated suffer from poorer health compared to their better educated counterparts; for instance, with respect to self-rated health or mortality (Gesthuizen et al., 2012; Mackenbach et al., 2008). While remaining largely stable over the life course, education is a major predictor of future life chances. It is strongly connected to access to resources important for health, such as material resources and social connections (Ellwardt et al., 2014).

The aim of this paper is to examine whether some aspects of doctor-patient relations exacerbate or compress health inequalities. Existing research points in this direction. Firstly, it has been shown that doctor-patient communication is affected by patient's SES in the sense that doctors convey less information and directions to patients from the lower social strata of a society, deploy a more directive consulting style, and give patients fewer positive socio-emotional responses (Willems et al., 2005). Secondly, aspects of doctor-patient relationships have been shown to be related to outcomes, such as patient satisfaction, coping with disease, and recall and understanding of information received from the physician (Ong et al., 1995), as well as to self-reported and objective health measures (Stewart, 1995a). However, there has been little research connecting the two issues of the SES-health gradient and SES differences in the doctor-patient relationship (Willems et al., 2005). Our study aims to fill this gap by explicitly addressing the role of doctor-patient relations in relation to educational inequalities in self-rated health.

An additional aspect that makes the doctor-patient relationship a particularly interesting factor in the study of inequalities in health is the current drastic transformations in these relationships. In part due to the growing awareness of the crucial role of doctor-patient relations for health outcomes, there are strong tendencies towards a more egalitarian relationship between physicians and their 
patients. The traditional 'doctor-centered' model (Byrne and Long, 1976), with its dominance of the doctor's role, is gradually being replaced with the 'patientcentered' model that gives greater weight to patients' needs and preferences, and aims at a partnership between the doctor and patient. As there are no empirical data on doctor-patient relations over a longer period of time, we increase the available variation in doctor-patient relations by studying 24 European countries for our analyses.

By addressing the role of patient-doctor relations in a model of the social gradient in health, we are aiming to extend research on health inequalities. Our central argument is that the differences in the doctor-patient relationship could affect the social gradient in health in two contrary ways. First, a more egalitarian relationship between doctors and patients might be accompanied by a more egalitarian distribution of health outcomes. Thus, an egalitarian doctor-patient relationship could potentially compress the social gradient in health. Second, differences in the doctor-patient relationship could potentially give rise to mechanisms that reproduce health inequalities. Whereas the traditional 'doctor-centered' model drew upon doctors' authority to induce compliance and to improve health, the new 'patient-centered' model builds upon patients' self-determination to generate positive health effects. The overall change in the doctor-patient relationship could lead to an advantage in health among highly educated individuals, due to their greater cognitive abilities and capacities to exercise self-regulation, exacerbating the social inequality in health.

The following section will elaborate upon the theoretical background of our study and generate hypotheses. This is followed by a description of the data and method used to test our hypotheses. The European Social Survey (2004/05) provides information on individuals in 24 European countries, with self-rated health examined via fixed and random effect models. Results are presented in the subsequent section, followed by a conclusion and reflection upon the results.

\subsection{Theoretical background}

\subsubsection{The doctor-patient relationship}

The relationship between doctors and patients is a complex phenomenon. It involves communication about issues of vital importance, but is often emotionally laden, sometimes involuntary, requires close cooperation, and is based upon interaction between individuals in unequal positions (Ong et al., 1995). Traditionally, the doctor - patient relationship is an asymmetric one, with the doctor being advantaged in several ways. Firstly, doctors command superior knowledge with 
regard to health and illness. Secondly, doctors have a gatekeeper function with respect to many aspects of health and illness, both in a formal (e.g. sickness certifications for employers) and an informal way (e.g. the sick role, Parsons, 1951). Thirdly, doctors have great power in steering interactions with patients, in terms of aspects, such as waiting times, but also regarding the content of meetings.

There is a long-standing tradition in sociology of research on the doctor-patient relationship (Henderson, 1935; Heritage and Maynard, 2006, 2011) and some classical works of sociology deal with the diffusion of innovations among physicians (Coleman et al., 1957) or interactions between physicians and patients (Parsons, 1951). However, there are relatively few studies which focus on the relationship between the doctor - patient relationship and health outcomes. A number of studies have shown that aspects of doctor-patient relationships can impact patient well-being and behavior, such as the ability to understand medical information, compliance with treatment regimes, coping with illness, satisfaction with care, overall quality of life, and health status (Griffin et al., 2004; O'Connor et al., 2009; Ong et al., 1995; Stewart, 1995a,b; Weiner et al., 2013; Williams et al., 2000).

The existing literature identified different pathways along which aspects of the doctor-patient relationship can impact health. One potential pathway is the quality of health care. A review of 139 studies revealed that the doctor-patient relationship is the most important predictor of patient-perceived quality of health care (Crow et al., 2003). Another possible pathway is adherence to doctors' recommendations. Stavropoulou (2011) shows that individuals' reports of adherence to medication recommendations depends on their relationships to their doctors, corroborating results of earlier studies (Barry et al., 2000; Bultman and Svarstad, 2000; Stavropoulou, 2012).

\subsubsection{Paternalistic and egalitarian models}

The asymmetry between doctors and patients is fundamental for different models of the doctor-patient relationship. Two different models are treated here, namely, the 'paternalistic' or 'doctor-centered' model and the 'egalitarian' or 'patient-centered' one. The paternalistic model actively builds upon the asymmetry between doctor and patient, as the doctor's role as an expert is crucial for this model to function. The general idea of the model is straightforward: Based on their superior medical knowledge, doctors give advice to their patients, who, in turn, comply with this advice. Driven by a desire to appear competent (White, 1959), i.e. to make correct decisions, and aiming at behavioral confirmation (Ormel et al., 1997), patients defer to the doctors' authority (Cialdini and Griskevicius, 2010). This authority is based on the doctors' expert status and their superior skills and knowledge. As 
the model greatly draws upon characteristics of the doctor while the patient holds a largely passive role, this model is sometimes called the 'doctor-centered model' (Byrne and Long, 1976).

In the last decades, however, the doctor-centered model is being replaced by the egalitarian model (Kaba and Sooriakumaran, 2007; Peck and Conner, 2011; Steinhart, 2002). This model tries to bridge the asymmetry between doctor and patient by also conceiving the patient as an expert, namely an expert for their own situation (Department of Health, 2001) and assigning them a more active role. This development has been fostered by campaigns encouraging patients to ask their physicians questions (Judson et al., 2013). Reasons for this shift of paradigms are numerous, with the most important ones being the crisis of the expert role in society (Beck, 1992), the greater availability of medical information for patients in self-help groups and internet sites (Neuberger, 2000), the increasing role of chronic, incurable diseases (Charmaz and Rosenfeld, 2010), and medical progress that has created more treatment options and, thus, more opportunities for decision-making in the cure process. The general idea of the model consists of multiple aspects: Both the doctor and patient are seen as experts - the doctor for conveying medical information, and the patient for their own preferences and living situation. The doctor's task is seen less as providing the patient with binding advice, but more to communicate medical information about diseases and treatments to the patient and to discuss the information with them, such as benefits and risks of different treatment options. If decisions are to be made, they will be made jointly (Charles et al., 1997), taking into account not only the medical facts, but also the patient's preferences.

The mechanism at the core of the patient-centered model is also different from the one underlying the doctor-centered one. Instead of satisfying the needs for competence and behavioral confirmation via complying with doctors' orders, the patient-centered model fulfills the desires for patient competence and autonomy by informing the patient and treating them as capable of deciding for themselves. According to self-determination theory (Djundeva et al., 2015; Ryan and Deci, 2000), a combination of competence and autonomy will induce intrinsic motivation in patients. Feelings of competence can be achieved via the provision of information, but these feelings of competence need to be accompanied with a sense of autonomy to result in intrinsic motivation (Deci et al., 1999). Intrinsic motivation is potentially of crucial importance in the cure process, as individuals with intrinsic motivation have more interest, confidence, and self-esteem, and perform better in comparison to individuals who are externally controlled (e.g. merely following orders). 


\subsubsection{Socioeconomic status and doctor-patient relations}

Previous research has shown that doctor-patient relations vary with patients' SES. Doctor-patient communication is affected by patient's SES in the sense that doctors convey less information and directions to patients from the lower social strata of a society, deploy a more directive consulting style, and give patients fewer positive socio-emotional responses (Willems et al., 2005). Also, doctors are able to identify the SES of their patients: Psychologist demonstrated that individuals can reliably identify the approximate income and SES of others within seconds (Kraus and Keltner, 2009).

One way doctor-patient relations could operate in the context of the educational gradient in self-rated health is that there might be an educational gradient in the doctor-patient relations. Given the evidence that higher SES might be associated with a different, more egalitarian model of doctor-patient relations (Willems et al., 2005) and the evidence suggesting that a more egalitarian model of doctorpatient relations is associated with better wellbeing and health outcomes (Griffin et al., 2004; O'Connor et al., 2009; Ong et al., 1995; Stewart, 1995a,b; Weiner et al., 2013; Williams et al., 2000), the relationship between education and selfrated health might be at least partially due to differences in the doctor-patient relationship. In line with this reasoning, we formulate the following expectation:

H1: An egalitarian doctor-patient relationship reduces the size of the positive association between education and self-rated health.

Additionally, the models of the doctor-patient relationship might have differing effects on different educational groups. In this respect, a group of particular interest is the lower educated, which is a prominent risk group for health marginalization (Gesthuizen et al., 2012). On the one hand, they might particularly benefit from a more patient-centered approach that takes their living conditions into account (e.g. Lutfey and Freese, 2005), but on the other, the paternalistic approach might be particularly detrimental for them, as it does not pay attention to special needs they might have. Based on this, we further hypothesize:

H2A: The lower educated benefit most in terms of self-rated health from an egalitarian doctor-patient relationship.

H2B: The lower educated suffer most in terms of self-rated health from a paternalistic doctor-patient relationship. 


\subsection{Data and methods}

\subsubsection{Data}

This study exploits the second round of the European Social Survey (ESS, Jowell et al., 2007), a large-scale survey conducted in 26 countries in 2004/05. We include 24 of these countries in our study, namely Austria (AT), Belgium (BE), Switzerland $(\mathrm{CH})$, the Czech Republic (CZ), Germany (DE), Denmark (DK), Estonia (EE), Spain (ES), Finland (FI), the United Kingdom (UK), Greece (GR), Hungary (HU), Italy (IT), Ireland (IE), Iceland (IS), Luxembourg (LU), the Netherlands (NL), Norway (NO), Poland (PL), Portugal (PT), Sweden (SE), and Slovenia (SI). Turkey and the Ukraine were excluded from the analysis, as first results revealed substantial cultural and socioeconomic differences to the other countries in the sample. In all countries, random probability samples were drawn, following standardized procedures and strict quality controls to ensure representativeness and comparability of the data.

Our study excludes respondents 25 years old or younger, as a substantial share of them has not yet finished education, which is one of our focal predictors in this study. Our sample size is further reduced by respondents who have missing values on any of the study variables. Descriptive statistics of the final sample of 31,089 respondents are provided in Table 5.1.

\section{Health outcome: Self-rated health}

The outcome variable of this study is self-rated health. Self-rated health was measured on a five-point scale ranging from 'very bad' (0) to 'very good' (4). Selfrated health is a general assessment of one's health status, not connected to any specific illness, but covering mental, physical, and social aspects of health (Idler et al., 1999). It has been shown to predict mortality and morbidity and has high test-retest reliability in a number of studies (Idler and Benyamini, 1997). Furthermore, this variable has been recommended by the WHO for comparative research (De Bruin et al., 1996) and a large number of researchers have followed this advice (Hildebrand and Van Kerm, 2009; Huijts and Kraaykamp, 2012). Research has also shown that different socioeconomic groups evaluate their health in comparable ways (Burström and Fredlund, 2001) and that the associations between objective health indicators and self-perceived health are largely similar across countries (Bardage et al., 2005). Clustering of self-rated health within countries is moderate by conventional standards: self-rated health has an intraclass correlation coefficient $(I C C)$ of .105 . The $I C C$ gives the proportion of the total variance in the dependent variable that is accounted for by the clustering in countries. Put differ- 
Table 5.1: Descriptive statistics (uncentered variables), $N=31,089$

\begin{tabular}{lcccc}
\hline Variable & Mean & Std. dev. & Min. & Max. \\
\hline Self-rated general health & 2.74 & 0.91 & 0 & 4 \\
Education & & & & \\
Low education & 0.34 & - & 0 & 1 \\
Medium education & 0.42 & - & 0 & 1 \\
High education & 0.24 & - & 0 & 1 \\
Egalitarian model & & & & \\
Easy to understand & 3.30 & 1.15 & 1 & 5 \\
Treat patients as equal & 3.20 & 1.26 & 1 & 5 \\
Discuss decisions & 3.33 & 1.23 & 1 & 5 \\
Paternalistic model & & & & \\
Patients reluctant to ask questions & 2.56 & 1.14 & 1 & 5 \\
Keep truth from patients & 2.25 & 1.07 & 1 & 5 \\
Doctors deny mistakes & 4.15 & 1.06 & 1 & 5 \\
Controls & & & & \\
Age & 50.8 & 15.50 & 26 & 101 \\
Female & 0.54 & - & 0 & 1 \\
Married/cohabiting & 0.70 & - & 0 & 1 \\
Active on labor market & 0.54 & - & 0 & 1 \\
\hline
\end{tabular}

ently, the $I C C$ is a measure of the extent to which respondents living in the same country are more similar to one another than to respondents living in other countries. An $I C C$ of .105 shows that nearly 90 per cent of the variation in self-rated health is found on the individual level.

\section{Predictor variables}

Doctor-patient relationship The ESS questionnaire contains six items covering different aspects of the doctor-patient relationship. Respondents were asked how often the statements applied to doctors in general. These six statements largely correspond to the two models of doctor-patient relations described previously and are reproduced in Table 5.2.

All answers are recorded on a five-point scale, ranging from 'never or almost never' (0) to 'always or almost always' (4). Zero-order correlations between the six items are low to moderate, and factor analyses do not yield a clear one- or two-dimensional structure. This points to the fact that the items capture distinct features of the doctor-patient relationship, but however does not allow for the creation of meaningful composite scores. 
Table 5.2: Paternalistic and egalitarian doctor-patient relationship as measured in the second round of the ESS

\section{Paternalistic model}

Patients are reluctant to ask their doctor all the questions they would like to ask.

Doctors keep the whole truth from their patients.

Doctors are not willing to admit their mistakes to their patients. (Item reversed)

\section{Egalitarian model}

Doctors use words or phrases that their patients do not find difficult to understand.

(Item reversed)

Doctors treat their patients as their equals.

Before doctors decide on a treatment, they discuss it with their patient.

Education We rely on education as our focal indicator of socioeconomic status. Based on the International Standard Classification of Education (UNESCO, 2006), we distinguish between three educational groups: low (ISCED 0-2), medium (ISCED 3-4), and the higher educated (ISCED 5-6). There are several reasons for relying on education for our purposes. Firstly, education reflects both individuals' material and non-material resources and their social status in a broad fashion. Secondly, the ISCED, with its high degree of cross-national standardization, allows to meaningfully compare educational groups across countries. Thirdly, educational attainment is usually completed in early adulthood and remains stable across the life course, thus reducing the chance of reverse causation: unlike occupational status, educational degrees do not change even when an adult experiences a health shock (Elstad, 2004). Fourthly, compared to income, which usually comes with a large proportion of non-respondents, education is a comparably easy-to-measure indicator of SES. Finally, education is a meaningful measure for the SES of both men and women (Lahelma, 2001).

Control variables As individual-level control variables, the models contain marital status/cohabitation $(0=$ not married or cohabiting with a partner, $1=$ married/cohabiting with a partner $)$, sex $(0=$ male, $1=$ female $)$, age, and employment status $(0=$ no paid work in last week, $1=$ paid work in last week $)$. Being married is a known correlate of many positive health outcomes (Koball et al., 2010) and there is evidence that it is not just the formal act of marriage that improves health, but rather that the presence of a partner is largely responsible for the positive impact (Meadows et al., 2008). In many countries, women are known to report slightly worse health compared to men (Dahlin and Härkönen, 2013). Higher age is known to be related with poorer health status, and paid work, regardless of full or part-time, improves and preserves health (Ross and Mirowsky, 1995). 


\subsubsection{Method and modeling strategy}

For analyzing a clustered dataset, multilevel (random effects) modeling is often recommended (Snijders and Boskers, 2012; Subramanian et al., 2003). Given the relatively low number of countries and our prime interest in the relationship between variables at the individual level (Bryan and Jenkins, 2015), we present results as obtained from OLS regression models. These models account for the country variance by including country fixed effects and correct for the clustering in the data by reporting cluster-robust (Huber-White) standard errors. We have centered our predictor variables at their respective country means, allowing us to obtain unbiased estimates of the within-country regressions (Enders and Tofighi, 2007). To assess the robustness of our analytical strategy, we have replicated our analyses using random effects modeling, leading to virtually identical results.

\subsection{Results}

Model 1 reported in Table 5.3 confirms the presence of an educational gradient in health. Respondents with medium and high education report significantly better health than lower educated respondents. A Wald test further confirms that the coefficients for medium and highly educated respondents differ from one another $(F(1,23)=134.51, p=.000)$, indicating that there is not a single dividing line between the lower educated and anyone with higher education, but that the health benefits of education increment gradually. The control variables in the model behave as can be expected from the literature: Older respondents and females report worse health (although the latter is not statistically significant), and those with a partner and those active on the labor market report better health. A comparison of the coefficients shows that (apart from age) education appears to be the most important predictor of health.

The indicators of an egalitarian doctor-patient relationship are entered in Model 2 of Table 5.3. Reporting that doctors are easy to understand and that they treat their patients as equals is associated with significantly better health, whereas the third indicator, whether doctors discuss their decisions with patients, is unrelated to self-reported health. Hypothesis 1, which had posited that a more egalitarian doctor-patient relationship attenuates the educational gradient in health, has to be rejected. When comparing the coefficients for education as obtained from Model 1 and Model 2, barely any difference can be detected, indicating that by and large, more egalitarian doctor-patient relations do not reduce educational differences in self-rated health.

In order to assess differences between countries for this finding, we have run 
Table 5.3: Self-rated health regressed on education and doctor-patient relationship, OLS regression

\begin{tabular}{|c|c|c|c|}
\hline & (1) & $(2)$ & (3) \\
\hline \multicolumn{4}{|l|}{ Education (ref. low education) } \\
\hline Medium education & $\begin{array}{l}0.167^{* * *} \\
(0.0196)\end{array}$ & $\begin{array}{l}0.162^{* * *} \\
(0.0196)\end{array}$ & $\begin{array}{l}0.166^{* * *} \\
(0.0201)\end{array}$ \\
\hline High education & $\begin{array}{l}0.301^{* * *} \\
(0.0209)\end{array}$ & $\begin{array}{l}0.293^{* * *} \\
(0.0214)\end{array}$ & $\begin{array}{l}0.297^{* * *} \\
(0.0215)\end{array}$ \\
\hline \multicolumn{4}{|l|}{ Egalitarian model } \\
\hline Easy to understand & & $\begin{array}{c}0.0196^{* * *} \\
(0.00508)\end{array}$ & \\
\hline Treat patients as equal & & $\begin{array}{c}0.0266^{* * *} \\
(0.00515)\end{array}$ & \\
\hline Discuss decisions & & $\begin{array}{l}-0.00149 \\
(0.00432)\end{array}$ & \\
\hline \multicolumn{4}{|l|}{ Paternalistic model } \\
\hline Patients reluctant to ask questions & & & $\begin{array}{c}-0.00539 \\
(0.00429)\end{array}$ \\
\hline Keep truth from patients & & & $\begin{array}{c}-0.0233^{* * *} \\
(0.00580)\end{array}$ \\
\hline Doctors deny mistakes & & & $\begin{array}{c}-0.0146^{*} \\
(0.00676)\end{array}$ \\
\hline Age & $\begin{array}{c}-0.0138^{* * *} \\
(0.00141)\end{array}$ & $\begin{array}{c}-0.0140^{* * *} \\
(0.00140)\end{array}$ & $\begin{array}{c}-0.0138^{* * *} \\
(0.00142)\end{array}$ \\
\hline Female (ref. male) & $\begin{array}{l}-0.0114 \\
(0.0176)\end{array}$ & $\begin{array}{l}-0.0105 \\
(0.0178)\end{array}$ & $\begin{array}{l}-0.0117 \\
(0.0175)\end{array}$ \\
\hline Married/cohab. (ref. not m./c.) & $\begin{array}{c}0.0801^{* * *} \\
(0.0126)\end{array}$ & $\begin{array}{c}0.0784^{* * *} \\
(0.0125)\end{array}$ & $\begin{array}{c}0.0798^{* * *} \\
(0.0125)\end{array}$ \\
\hline Active on labor market (ref. not active) & $\begin{array}{l}0.286^{* * *} \\
(0.0178)\end{array}$ & $\begin{array}{l}0.286^{* * *} \\
(0.0176)\end{array}$ & $\begin{array}{l}0.285^{* * *} \\
(0.0178)\end{array}$ \\
\hline Intercept & $\begin{array}{l}2.871^{* * *} \\
(0.0144)\end{array}$ & $\begin{array}{l}2.875^{* * *} \\
(0.0147)\end{array}$ & $\begin{array}{l}2.874^{* * *} \\
(0.0147)\end{array}$ \\
\hline Country dummies included & YES & YES & YES \\
\hline $\begin{array}{l}N \text { countries } \\
N\end{array}$ & $\begin{array}{c}24 \\
31,089\end{array}$ & $\begin{array}{c}24 \\
31,089\end{array}$ & $\begin{array}{c}24 \\
31,089\end{array}$ \\
\hline
\end{tabular}

Notes: Robust standard errors in parentheses. Coefficients for country dummies not displayed.

${ }^{*} p<0.05,{ }^{* *} p<0.01,{ }^{* * *} p<0.001$ 
separate regression models by country and report the education coefficients before and after adjusting for the egalitarian doctor-patient relationship in Figure 5.1. While it is apparent that the size of the educational gradient in health varies considerably across countries, closer inspection reveals that differences between the adjusted and unadjusted coefficients are negligible, indicating that the finding of no meaningful reduction in the educational gradient in health in case of a more egalitarian doctor-patient relationship holds for all countries under study.

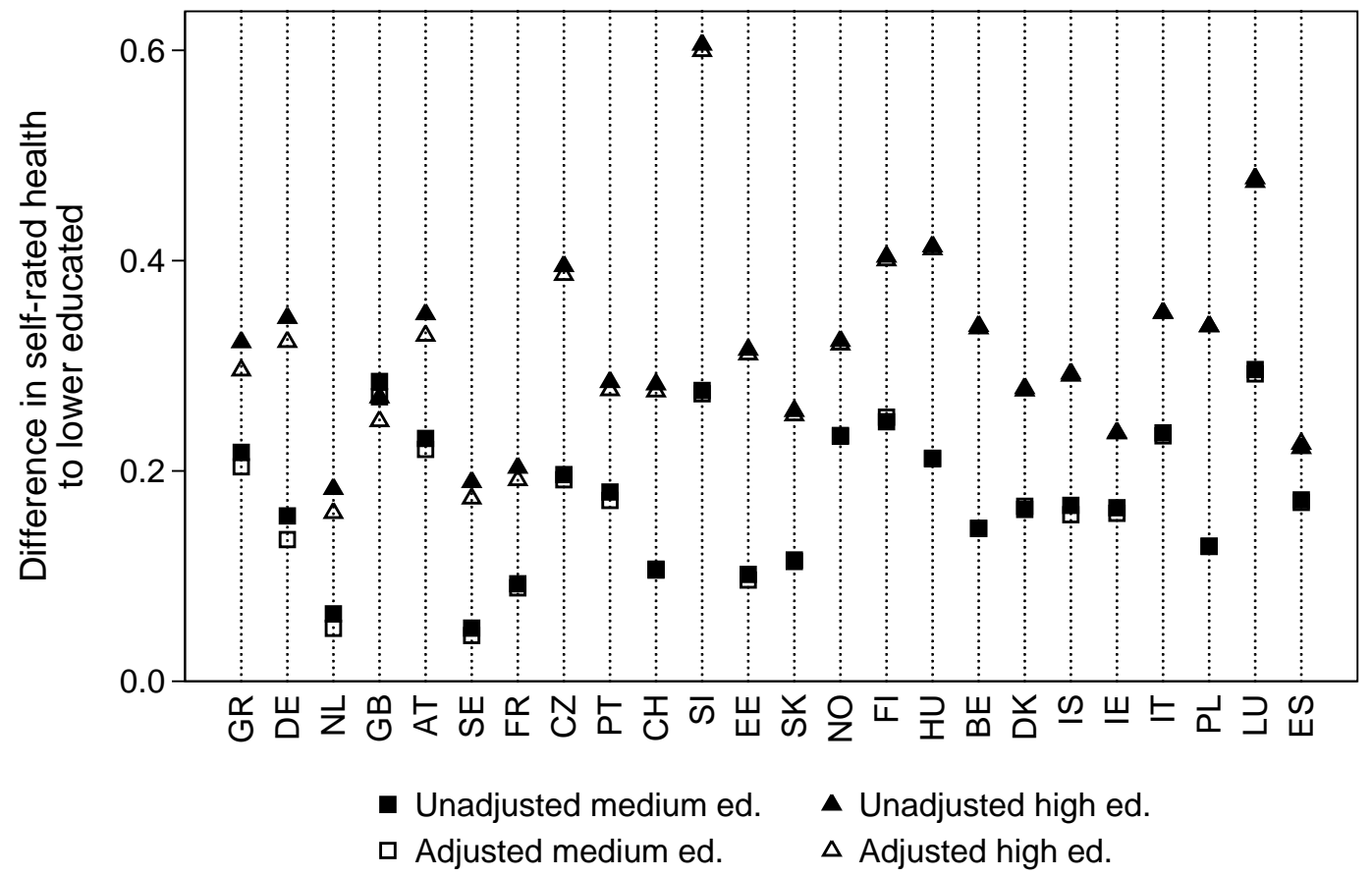

Figure 5.1: Educational gradient in health by country, before and after adjusting for egalitarian doctor-patient relationship, based on country-specific OLS regression models

Note: All models control for age, sex, marital status, and labor market status.

For the indicators of a paternalistic doctor-patient relationship, Model 3 of Table 5.3 indicates direct negative associations with self-rated health for two of the three indicators. When patients report that doctors keep the truth from patients and deny mistakes, it goes along with statistically significant poorer self-rated health. Reporting about a reluctance to ask questions has a negative effect that is not statistically different from zero. The size of the educational gradient in health is, however, unaffected by including indicators of a paternalistic doctor-patient relationship, as can be seen from comparing the coefficients for education between Models 2 and 3.

Table 5.4 reports the interactions between indicators of the doctor-patient relationship and education. For two of the six indicators, we can detect a statistically 
significant interaction with education, namely when respondents report doctors to be easy to understand (Model 4 of Table 5.4) and when patients are reluctant to ask questions (Model 7 of Table 5.4). To facilitate interpretation, we have plotted the interactions in Figure 5.2. The upper panel of Figure 5.2 plots the interaction of Model 4 of Table 5.4. It shows that whether doctors are easy to understand plays virtually no role for the self-rated health of the higher and medium educated; the slopes of their predicted lines are virtually flat over the continuum of how easy doctors are to understand. For the group of the lower educated, however, we can see a marked increase in self-rated health as the reports of doctors being easy to understand increase. The plot shows evidence that it is the lower educated who benefit from a more egalitarian doctor-patient relationship, giving some support for Hypothesis 2A. The lower panel of Figure 5.2 plots the interaction of Model 7 of Table 5.4. Again, for the higher educated and for those with medium education, it shows that there is virtually no association of self-rated health with reporting that patients are reluctant to ask questions, as the slopes of the predictions are flat. However, for the lower educated, we can see a falling slope as the agreement to the statement that patients are reluctant to ask questions gets stronger. Substantively, this means that a reluctance to answer questions is irrelevant for those with educational degrees classified as medium and high, but for the lower educated, such a reported reluctance is associated with worse self-rated health. This gives some support for Hypothesis 2B. 
Table 5.4: Self-rated health regressed on education and doctor-patient relationship including interaction terms, OLS regression

\begin{tabular}{|c|c|c|c|c|c|c|}
\hline & (4) & (5) & (6) & (7) & (8) & (9) \\
\hline \multicolumn{7}{|l|}{ Education (ref. low education) } \\
\hline Medium education & $\begin{array}{l}0.161^{* * *} \\
(0.0200)\end{array}$ & $\begin{array}{l}0.162^{* * *} \\
(0.0197)\end{array}$ & $\begin{array}{l}0.163^{* * *} \\
(0.0196)\end{array}$ & $\begin{array}{l}0.166^{* * *} \\
(0.0201)\end{array}$ & $\begin{array}{l}0.165^{* * *} \\
(0.0198)\end{array}$ & $\begin{array}{l}0.166^{* * *} \\
(0.0200)\end{array}$ \\
\hline High education & $\begin{array}{l}0.293^{* * *} \\
(0.0215)\end{array}$ & $\begin{array}{l}0.292^{* * *} \\
(0.0214)\end{array}$ & $\begin{array}{l}0.292^{* * *} \\
(0.0215)\end{array}$ & $\begin{array}{l}0.298^{* * *} \\
(0.0216)\end{array}$ & $\begin{array}{l}0.297^{* * *} \\
(0.0213)\end{array}$ & $\begin{array}{l}0.298^{* * *} \\
(0.0215)\end{array}$ \\
\hline \multicolumn{7}{|l|}{ Egalitarian model } \\
\hline Easy to understand & $\begin{array}{l}0.0358^{* * *} \\
(0.00917)\end{array}$ & $\begin{array}{l}0.0196^{* * *} \\
(0.00511)\end{array}$ & $\begin{array}{l}0.0197^{* * *} \\
(0.00510)\end{array}$ & & & \\
\hline Medium ed. $\times$ Easy to understand & $\begin{array}{l}-0.0227^{*} \\
(0.0100)\end{array}$ & & & & & \\
\hline High ed. $\times$ Easy to understand & $\begin{array}{c}-0.0326^{*} \\
(0.0119)\end{array}$ & & & & & \\
\hline Treat patients as equal & $\begin{array}{l}0.0267^{* * *} \\
(0.00513)\end{array}$ & $\begin{array}{l}0.0331^{* * *} \\
(0.00842)\end{array}$ & $\begin{array}{l}0.0266^{* * *} \\
(0.00514)\end{array}$ & & & \\
\hline Medium ed. $\times$ Treat patients as equal & & $\begin{array}{l}-0.00938 \\
(0.00825)\end{array}$ & & & & \\
\hline High ed. $\times$ Treat patients as equal & & $\begin{array}{l}-0.0114 \\
(0.0100)\end{array}$ & & & & \\
\hline Discuss decisions & $\begin{array}{l}-0.00116 \\
(0.00432)\end{array}$ & $\begin{array}{l}-0.00139 \\
(0.00433)\end{array}$ & $\begin{array}{c}0.00439 \\
(0.00769)\end{array}$ & & & \\
\hline Medium ed. $\times$ Discuss decisions & & & $\begin{array}{l}-0.00846 \\
(0.0109)\end{array}$ & & & \\
\hline High ed. $\times$ Discuss decisions & & & $\begin{array}{r}-0.00996 \\
(0.0106)\end{array}$ & & & \\
\hline \multicolumn{7}{|l|}{ Paternalistic model } \\
\hline Patients reluctant to ask questions & & & & $\begin{array}{l}-0.0209^{* *} \\
(0.00579)\end{array}$ & $\begin{array}{l}-0.00536 \\
(0.00429)\end{array}$ & $\begin{array}{l}-0.00531 \\
(0.00430)\end{array}$ \\
\hline Medium ed. $\times$ Patients reluctant to $\ldots$ & & & & $\begin{array}{l}0.0214^{*} \\
(0.00817)\end{array}$ & & \\
\hline High ed. $\times$ Patients reluctant to $\ldots$ & & & & $\begin{array}{l}0.0293^{* *} \\
(0.00823)\end{array}$ & & \\
\hline Keep truth from patients & & & & $\begin{array}{c}-0.0232^{* * *} \\
(0.00582)\end{array}$ & $\begin{array}{l}-0.0275^{*} \\
(0.0107)\end{array}$ & $\begin{array}{r}-0.0233^{* * *} \\
(0.00581)\end{array}$ \\
\hline Medium ed. $\times$ Keep truth from $\ldots$ & & & & & $\begin{array}{l}0.00394 \\
(0.0128)\end{array}$ & \\
\hline High ed. $\times$ Keep truth from $\ldots$ & & & & & $\begin{array}{c}0.0120 \\
(0.0106)\end{array}$ & \\
\hline Doctors deny mistakes & & & & $\begin{array}{l}-0.0147^{*} \\
(0.00673)\end{array}$ & $\begin{array}{l}-0.0146^{*} \\
(0.00675)\end{array}$ & $\begin{array}{c}-0.0132 \\
(0.00991)\end{array}$ \\
\hline Medium ed. $\times$ Doctors deny mistakes & & & & & & $\begin{array}{l}0.00170 \\
(0.0120)\end{array}$ \\
\hline High ed. $\times$ Doctors deny mistakes & & & & & & $\begin{array}{r}-0.00911 \\
(0.0134)\end{array}$ \\
\hline Age & $\begin{array}{r}-0.0140^{* * *} \\
(0.00140)\end{array}$ & $\begin{array}{r}-0.0140^{* * *} \\
(0.00140)\end{array}$ & $\begin{array}{c}-0.0140^{* * *} \\
(0.00141)\end{array}$ & $\begin{array}{c}-0.0138^{* * *} \\
(0.00142)\end{array}$ & $\begin{array}{c}-0.0138^{* * *} \\
(0.00142)\end{array}$ & $\begin{array}{r}-0.0138^{* * *} \\
(0.00142)\end{array}$ \\
\hline Female (ref. male) & $\begin{array}{l}-0.0106 \\
(0.0178)\end{array}$ & $\begin{array}{l}-0.0106 \\
(0.0178)\end{array}$ & $\begin{array}{l}-0.0106 \\
(0.0178)\end{array}$ & $\begin{array}{l}-0.0124 \\
(0.0175)\end{array}$ & $\begin{array}{l}-0.0117 \\
(0.0175)\end{array}$ & $\begin{array}{l}-0.0117 \\
(0.0175)\end{array}$ \\
\hline Married/cohab. (ref. not m./c.) & $\begin{array}{c}0.0781^{* * *} \\
(0.0125)\end{array}$ & $\begin{array}{c}0.0784^{* * *} \\
(0.0125)\end{array}$ & $\begin{array}{c}0.0784^{* * *} \\
(0.0125)\end{array}$ & $\begin{array}{l}0.0800^{* * *} \\
(0.0125)\end{array}$ & $\begin{array}{c}0.0798^{* * *} \\
(0.0125)\end{array}$ & $\begin{array}{c}0.0798^{* * *} \\
(0.0125)\end{array}$ \\
\hline Active on labor market (ref. not a.) & $\begin{array}{l}0.286^{* * *} \\
(0.0176)\end{array}$ & $\begin{array}{l}0.286^{* * *} \\
(0.0177)\end{array}$ & $\begin{array}{l}0.286^{* * *} \\
(0.0177)\end{array}$ & $\begin{array}{l}0.285^{* * *} \\
(0.0179)\end{array}$ & $\begin{array}{l}0.285^{* * *} \\
(0.0178)\end{array}$ & $\begin{array}{l}0.285^{* * *} \\
(0.0178)\end{array}$ \\
\hline Intercept & $\begin{array}{l}2.877^{* * *} \\
(0.0151)\end{array}$ & $\begin{array}{l}2.876^{* * *} \\
(0.0147)\end{array}$ & $\begin{array}{l}2.875^{* * *} \\
(0.0147)\end{array}$ & $\begin{array}{l}2.873^{* * *} \\
(0.0147)\end{array}$ & $\begin{array}{l}2.874^{* * *} \\
(0.0143)\end{array}$ & $\begin{array}{l}2.874^{* * *} \\
(0.0147)\end{array}$ \\
\hline Country dummies included & YES & YES & YES & YES & YES & YES \\
\hline$N$ countries & 24 & 24 & 24 & 24 & 24 & 24 \\
\hline$N$ & 31,089 & 31,089 & 31,089 & 31,089 & 31,089 & 31,089 \\
\hline
\end{tabular}

Notes: Robust standard errors in parentheses. Coefficients for country dummies not displayed.

${ }^{*} p<0.05,{ }^{* *} p<0.01,{ }^{* * *} p<0.001$ 


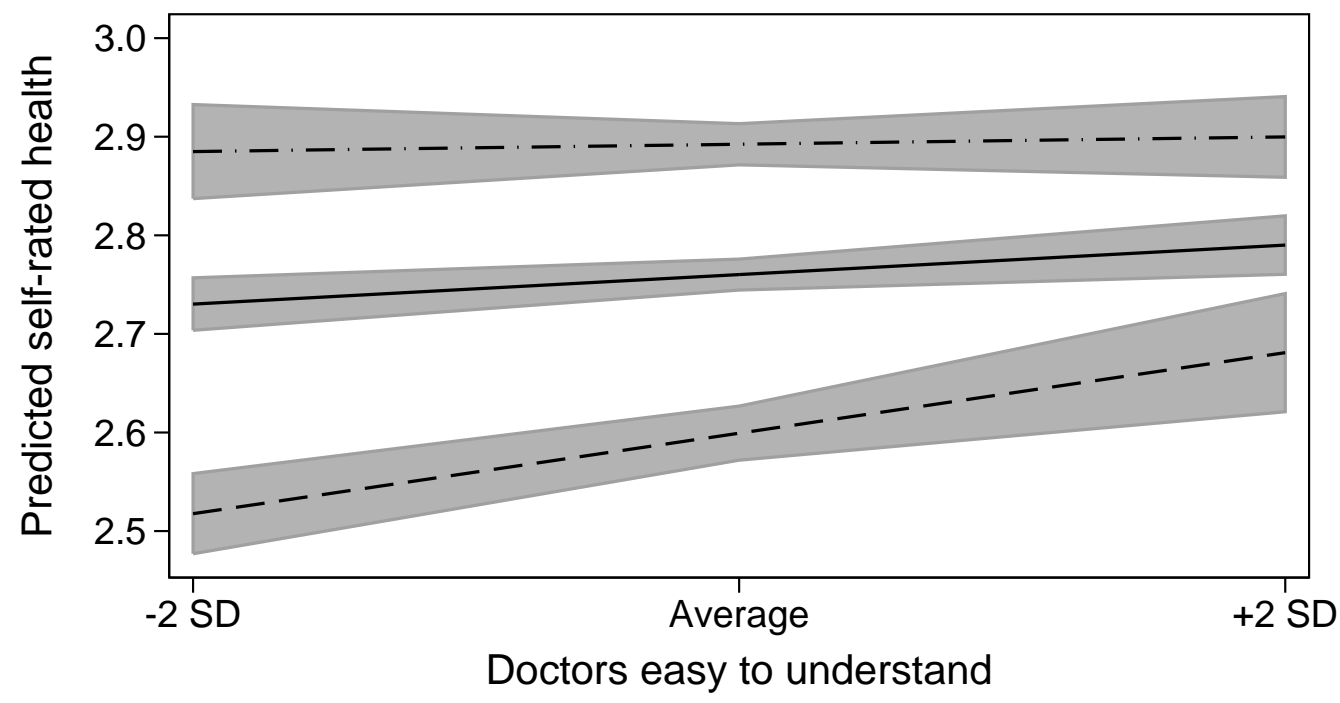

- - Higher $\quad$ Medium $\quad-$ Lower educated

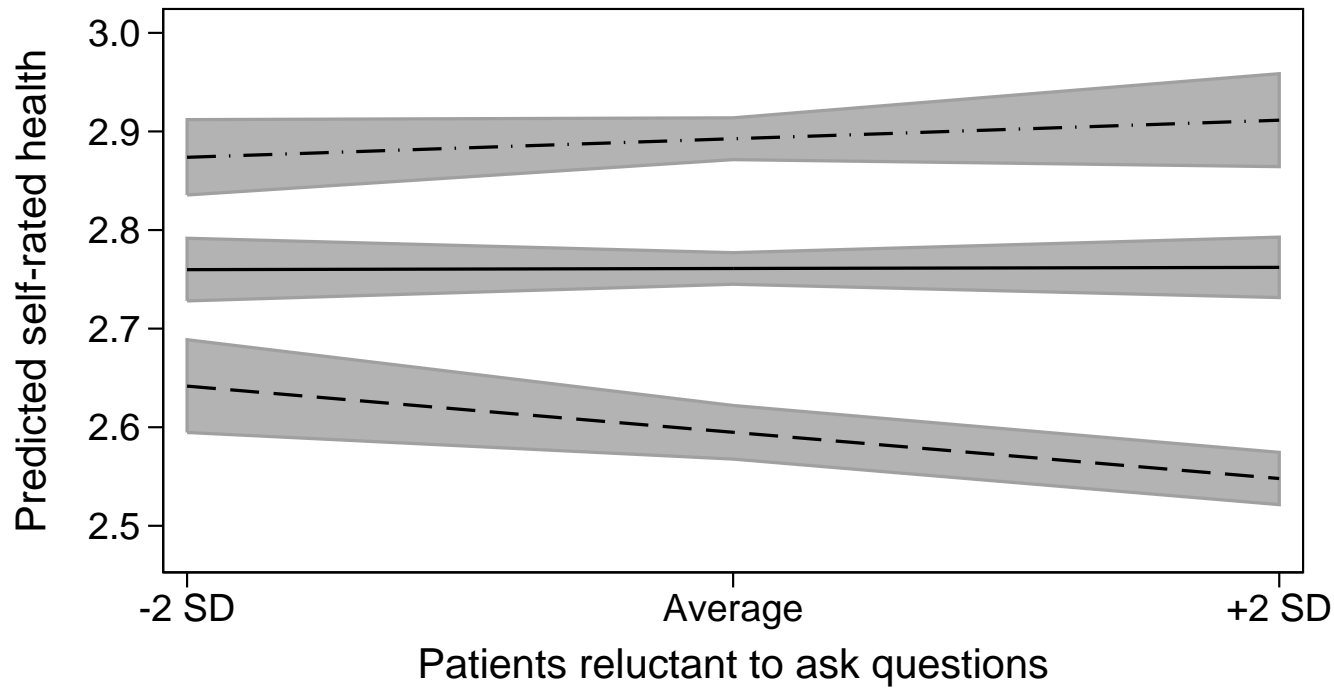

- - Higher $\longrightarrow$ Medium $\quad-$ Lower educated

$95 \%$ confidence intervals based on robust standard errors

Figure 5.2: Upper panel: Plot of the 'doctors easy to understand' $\times$ education interaction. Lower panel: Plot of the 'patients reluctant to ask questions' $\times$ education interaction.

Note: Calculations based on Model 4 of Table 5.4 (upper panel) and Model 7 of Table 5.4 (lower panel). 


\subsubsection{Sensitivity analyses}

We were able to replicate these findings from pooled OLS models with country dummies and robust standard errors also using random effects models without obtaining substantially different results. Findings from these analyses are reported in the Appendix of this chapter.

\subsection{Discussion}

The role of doctor-patient relations for the educational gradient in self-rated health is largely unknown. Drawing on a large, European general population survey, our study contributed to this field of research in several respects.

Firstly, our analyses showed that two different models of the doctor-patient relationship, namely the traditional paternalistic model and the more egalitarian, 'patient-centered' model are directly associated with self-rated health. Reports that doctors are easy to understand and treat their patients as equals go along with better self-rated health. Conversely, reports that doctors do not tell the whole truth and are not willing to admit mistakes is associated with worse self-ratings of health. This confirms findings from previous studies, which have suggested that a more egalitarian doctor-patient relationship is associated with better wellbeing and health outcomes (Griffin et al., 2004; O'Connor et al., 2009; Ong et al., 1995; Stewart, 1995a,b; Weiner et al., 2013; Williams et al., 2000).

Secondly, the different models of the doctor-patient relationship do not mediate the educational gradient in self-rated health. Despite the evidence for differential treatment of patients by their doctors according to their SES (Willems et al., 2005), accounting for those differences does not decrease the education-self-rated health association. This has important policy implications, as measures to improve doctor-patient relations can have a positive impact on overall population health, but cannot be expected to compress the social gradient in health.

Thirdly, we found a number of interactions between education and indicators of the different models of doctor-patient relations. While for those with medium and high educational degrees reporting that doctors are easy to understand - an indicator of the egalitarian model of doctor-patient relations - does not translate into better health, but for the lower educated, reporting that doctors are easy to understand is associated with better self-rated health. Also, reports that patients are reluctant to ask questions - an indicator of the paternalistic model of the doctor-patient relationship - is unrelated to self-rated health for the medium and higher educated, but for the lower educated there is a negative association. When the lower educated report that patients are reluctant to ask questions, this 
is associated with lower self-rated health. Thus, some aspects of the transition of the doctor-patient relationship towards a more egalitarian and a less paternalistic model could improve the health of the lower educated.

We acknowledge that our conclusions are based on a cross-sectional, observational study. Our results are associational and cannot make any claims about causality. Despite these caveats, we believe that our study gives important impulses for future research under improved methodological conditions. Drawing on longitudinal data would help to rule out causality issues and could yield greater insights as to how the change in doctor-patient relations occurred over time. Also, using validated and more comprehensive indicators of the different models of the doctor-patient relationship could strengthen the case for the arguments laid out in this chapter. Despite these limitations, our study was the first to reveal that in a large European population sample the move towards a more egalitarian model of doctor-patient relations did not have the positive effects on social inequality in health that one could have expected, which opens up avenues for further research on the social gradient in health.

\section{Appendix}

This Appendix includes replications of the analyses reported in the main text of the chapter using random effects models. Table A5.1 is a replication of the models examining the direct and indirect effects reported in Table 5.3 and Table A5.2 reports the results of a replication of the moderation analyses reported in Table 5.4. A comparison of the Tables shows that the results are robust to these different estimation strategies. 
Table A5.1: Self-rated health regressed on education and doctor-patient relationship, random intercept models

(1)

Education (ref. low education)

Medium education

High education

Egalitarian model

Easy to understand

Treat patients as equal

Discuss decisions

Paternalistic model

Patients reluctant to ask questions

$\begin{array}{lll}0.167^{* * *} & 0.162^{* * *} & 0.165^{* * *} \\ (0.0120) & (0.0120) & (0.0120) \\ 0.301^{* * *} & 0.293^{* * *} & 0.297^{* * *} \\ (0.0133) & (0.0134) & (0.0133)\end{array}$

(2)

(3)

(0.0133)
$0.0196^{* * *}$

(0.00402)

$0.0266^{* * *}$

$(0.00403)$

$-0.00148$

(0.00417)
$-0.00539$

Keep truth from patients

(0.00410)

$-0.0233^{* * *}$

(0.00436)

Doctors deny mistakes

$-0.0146^{* * *}$

(0.00428)

Age

$-0.0138^{* * *}$

$-0.0140^{* * *}$

$-0.0138^{* * *}$

Female (ref. male)

$(0.000354) \quad(0.000355)$

$(0.000354)$

$-0.0115$

$-0.0106$

$-0.0117$

Married/cohab. (ref. not m./c.)

$(0.00921)$

$(0.00920)$

$(0.00921)$

$0.0800^{* * *}$

$0.0784^{* * *}$

$0.0798^{* * *}$

(0.00990)

Active on labor market (ref. not active)

$0.286^{* * *}$

$(0.00989)$

(0.00989)

(0.0110)

$0.286^{* * *}$

$0.285^{* * *}$

(0.0109)

(0.0110)

Intercept

$2.615^{* * *}$

$2.622^{* * *}$

$2.618^{* * *}$

$(0.0568)$

(0.0567)

$(0.0569)$

\begin{tabular}{lccc}
\hline Variance (intercept) & $0.0754^{* * *}$ & $0.0752^{* * *}$ & $0.756^{* * *}$ \\
Variance (residual) & $0.625^{* * *}$ & $0.623^{* * *}$ & $0.624^{* * *}$ \\
\hline$N$ countries & 24 & 24 & 24 \\
$N$ & 31,089 & 31,089 & 31,089 \\
\hline Notes: Standard errors in parentheses. ${ }^{*} p<0.05,^{* *} p<0.01,{ }^{* * *} p<0.001$
\end{tabular}

Notes: Standard errors in parentheses. ${ }^{*} p<0.05,{ }^{* *} p<0.01,{ }^{* * *} p<0.001$ 
Table A5.2: Self-rated health regressed on education and doctor-patient relationship including interaction terms, random intercept models

\begin{tabular}{|c|c|c|c|c|c|c|}
\hline & (4) & $(5)$ & (6) & (7) & (8) & (9) \\
\hline \multicolumn{7}{|l|}{ Education (ref. low education) } \\
\hline Medium education & $\begin{array}{l}0.160^{* * *} \\
(0.0120)\end{array}$ & $\begin{array}{l}0.161^{* * *} \\
(0.0120)\end{array}$ & $\begin{array}{l}0.162^{* * *} \\
(0.0120)\end{array}$ & $\begin{array}{l}0.166^{* * *} \\
(0.0120)\end{array}$ & $\begin{array}{l}0.165^{* * *} \\
(0.0120)\end{array}$ & $\begin{array}{l}0.165^{* * *} \\
(0.0120)\end{array}$ \\
\hline High education & $\begin{array}{l}0.293^{* * *} \\
(0.0134)\end{array}$ & $\begin{array}{l}0.292^{* * *} \\
(0.0134)\end{array}$ & $\begin{array}{l}0.293^{* * *} \\
(0.0134)\end{array}$ & $\begin{array}{l}0.298^{* * *} \\
(0.0133)\end{array}$ & $\begin{array}{l}0.297^{* * *} \\
(0.0134)\end{array}$ & $\begin{array}{l}0.298^{* * *} \\
(0.0133)\end{array}$ \\
\hline \multicolumn{7}{|l|}{ Egalitarian model } \\
\hline Easy to understand & $\begin{array}{l}0.0358^{* * *} \\
(0.00651)\end{array}$ & $\begin{array}{l}0.0196^{* * *} \\
(0.00402)\end{array}$ & $\begin{array}{l}0.0197^{* * *} \\
(0.00403)\end{array}$ & & & \\
\hline Medium ed. $\times$ Easy to understand & $\begin{array}{l}-0.0227^{*} \\
(0.00893)\end{array}$ & & & & & \\
\hline High ed. $\times$ Easy to understand & $\begin{array}{c}-0.0325^{* *} \\
(0.0109)\end{array}$ & & & & & \\
\hline Treat patients as equal & $\begin{array}{l}0.0267^{* * *} \\
(0.00402)\end{array}$ & $\begin{array}{l}0.0331^{* * *} \\
(0.00631)\end{array}$ & $\begin{array}{l}0.0267^{* * *} \\
(0.00403)\end{array}$ & & & \\
\hline Medium ed. $\times$ Treat patients as equal & & $\begin{array}{l}-0.00934 \\
(0.00834)\end{array}$ & & & & \\
\hline High ed. $\times$ Treat patients as equal & & $\begin{array}{c}-0.0114 \\
(0.00971)\end{array}$ & & & & \\
\hline Discuss decisions & $\begin{array}{l}-0.00116 \\
(0.00417)\end{array}$ & $\begin{array}{l}-0.00139 \\
(0.00417)\end{array}$ & $\begin{array}{c}0.00440 \\
(0.00667)\end{array}$ & & & \\
\hline Medium ed. $\times$ Discuss decisions & & & $\begin{array}{l}-0.00844 \\
(0.00872)\end{array}$ & & & \\
\hline High ed. $\times$ Discuss decisions & & & $\begin{array}{l}-0.01000 \\
(0.0101)\end{array}$ & & & \\
\hline \multicolumn{7}{|l|}{ Paternalistic model } \\
\hline Patients reluctant to ask questions & & & & $\begin{array}{l}-0.0210^{* *} \\
(0.00677)\end{array}$ & $\begin{array}{l}-0.00537 \\
(0.00410)\end{array}$ & $\begin{array}{l}-0.00532 \\
(0.00410)\end{array}$ \\
\hline Medium ed. $\times$ Patients reluctant to $\ldots$ & & & & $\begin{array}{c}0.0215^{*} \\
(0.00917)\end{array}$ & & \\
\hline High ed. $\times$ Patients reluctant to $\ldots$ & & & & $\begin{array}{l}0.0294^{* *} \\
(0.0108)\end{array}$ & & \\
\hline Keep truth from patients & & & & $\begin{array}{r}-0.0232^{* * *} \\
(0.00436)\end{array}$ & $\begin{array}{c}-0.0275^{* * *} \\
(0.00698)\end{array}$ & $\begin{array}{r}-0.0233^{* * *} \\
(0.00436)\end{array}$ \\
\hline Medium ed. $\times$ Keep truth from $\ldots$ & & & & & $\begin{array}{c}0.00397 \\
(0.00968)\end{array}$ & \\
\hline High ed. $\times$ Keep truth from $\ldots$ & & & & & $\begin{array}{c}0.0120 \\
(0.0115)\end{array}$ & \\
\hline Doctors deny mistakes & & & & $\begin{array}{r}-0.0147^{* * *} \\
(0.00428)\end{array}$ & $\begin{array}{c}-0.0146^{* * *} \\
(0.00428)\end{array}$ & $\begin{array}{c}-0.0132 \\
(0.00693)\end{array}$ \\
\hline Medium ed. $\times$ Doctors deny mistakes & & & & & & $\begin{array}{c}0.00167 \\
(0.00970)\end{array}$ \\
\hline High ed. $\times$ Doctors deny mistakes & & & & & & $\begin{array}{r}-0.00909 \\
(0.0113)\end{array}$ \\
\hline Age & $\begin{array}{l}-0.0141^{* * *} \\
(0.000355)\end{array}$ & $\begin{array}{l}-0.0140^{* * *} \\
(0.000355)\end{array}$ & $\begin{array}{l}-0.0140^{* * *} \\
(0.000355)\end{array}$ & $\begin{array}{l}-0.0138^{* * *} \\
(0.000354)\end{array}$ & $\begin{array}{l}-0.0138^{* * *} \\
(0.000354)\end{array}$ & $\begin{array}{l}-0.0138^{* * *} \\
(0.000354)\end{array}$ \\
\hline Female (ref. male) & $\begin{array}{l}-0.0106 \\
(0.00920)\end{array}$ & $\begin{array}{l}-0.0107 \\
(0.00920)\end{array}$ & $\begin{array}{c}-0.0107 \\
(0.00920)\end{array}$ & $\begin{array}{l}-0.0125 \\
(0.00921)\end{array}$ & $\begin{array}{l}-0.0118 \\
(0.00921)\end{array}$ & $\begin{array}{c}-0.0118 \\
(0.00921)\end{array}$ \\
\hline Married/cohab. (ref. not m./c.) & $\begin{array}{l}0.0781^{* * *} \\
(0.00989)\end{array}$ & $\begin{array}{l}0.0784^{* * *} \\
(0.00989)\end{array}$ & $\begin{array}{l}0.0783^{* * *} \\
(0.00989)\end{array}$ & $\begin{array}{l}0.0799^{* * *} \\
(0.00989)\end{array}$ & $\begin{array}{l}0.0797^{* * *} \\
(0.00989)\end{array}$ & $\begin{array}{l}0.0797^{* * *} \\
(0.00989)\end{array}$ \\
\hline Active on labor market (ref. not a.) & $\begin{array}{l}0.286^{* * *} \\
(0.0109)\end{array}$ & $\begin{array}{l}0.286^{* * *} \\
(0.0109)\end{array}$ & $\begin{array}{l}0.286^{* * *} \\
(0.0109)\end{array}$ & $\begin{array}{l}0.285^{* * *} \\
(0.0110)\end{array}$ & $\begin{array}{l}0.285^{* * *} \\
(0.0110)\end{array}$ & $\begin{array}{l}0.285^{* * *} \\
(0.0110)\end{array}$ \\
\hline Intercept & $\begin{array}{l}2.623^{* * *} \\
(0.0568)\end{array}$ & $\begin{array}{l}2.622^{* * *} \\
(0.0568)\end{array}$ & $\begin{array}{l}2.621^{* * *} \\
(0.0567)\end{array}$ & $\begin{array}{l}2.617^{* * *} \\
(0.0568)\end{array}$ & $\begin{array}{l}2.618^{* * *} \\
(0.0569)\end{array}$ & $\begin{array}{l}2.618^{* * *} \\
(0.0569)\end{array}$ \\
\hline Variance (intercept) & $0.0752^{* * *}$ & $0.0752^{* * *}$ & $0.0752^{* * *}$ & $0.0754^{* * *}$ & $0.0756^{* * *}$ & $0.0756^{* * *}$ \\
\hline Variance (residual) & $0.623^{* * *}$ & $0.623^{* * *}$ & $0.623^{* * *}$ & $0.624^{* * * *}$ & $0.624^{* * *}$ & $0.624^{* * *}$ \\
\hline $\begin{array}{l}N \text { countries } \\
N\end{array}$ & $\begin{array}{c}24 \\
31,089\end{array}$ & $\begin{array}{c}24 \\
31,089\end{array}$ & $\begin{array}{c}24 \\
31,089\end{array}$ & $\begin{array}{c}24 \\
31,089\end{array}$ & $\begin{array}{c}24 \\
31,089\end{array}$ & $\begin{array}{c}24 \\
31,089\end{array}$ \\
\hline
\end{tabular}

Notes:Robust standard errors in parentheses. ${ }^{*} p<0.05,{ }^{* *} p<0.01,{ }^{* * *} p<0.001$ 



\section{Chapter 6}

\section{Conclusion}

The existence of social inequalities in health does not only raise import ethical questions (Daniels, 2008), it also suggests potential for improving overall population health by enhancing the health status of those who are worse off and, thus, reducing health inequalities. Improving the health status of populations is an important policy goal, and better understanding the linkages between social stratification and health can identify key levers for doing so. However, the multifaceted nature of socioeconomic status (SES) and the multilevel nature upon which it operates makes it difficult to find simple solutions.

In four empirical Chapters, the present book examined a number of pathways that lead from aspects of social stratification to health. After demonstrating the remarkable variability of health gradients not only between, but also within countries, it proceeded to explore how subjective aspects of SES affected health, taking into account how these effects are modified by the social conditions in societies. Having shown that subjective perceptions of SES affect health in a large number of countries, the role of social comparisons for health is examined to get a closer understanding of how subjective aspects of SES affect health. An additional aspect of health inequalities are inequalities in power relationships in health care. To shed light on the SES aspects of this important area, the role of the doctor-patient relationship, which is characterized by an imbalance in terms of power between the doctor and the patient, was studied with respect to its associations with health inequalities.

In this concluding Chapter, the main findings are summarized first, then the theoretical and practical implications are discussed, the contributions of the studies are reviewed, and, finally, limitations and directions for future research are identified. 


\subsection{Summary of main findings}

\subsubsection{Educational health gradients in the US and Europe}

The previous Chapters revealed a number of key findings. Chapter 2 explored the variation in the educational gradient in health across the countries of Europe and the federal states of the US. The first remarkable finding of the study was the great variability of the relationship between education and self-rated health across the European countries and the federal states. But we did not compare the relationship only between countries, for one select country - the US - we had an especially large sample. With these data, we were also able to look into the education-health relationship on a subnational level, namely on the level of the 50 federal states. Doing so revealed the great heterogeneity in the association between education and health within a single country. The within-US patterns were striking when seen in conjunction with the findings from Europe: Despite the high per capita income in the US, some regions in the US reveal health inequalities similar to those found in Eastern European middle income countries.

\subsubsection{Subjective SES-health gradients in comparative per- spective}

Chapter 3 took our analysis further in the direction of the subjective aspects of SES. Drawing on information from 27 high and middle income countries from around the world, we explored the relationship between subjective SES, one's appraisal about their own location in a socioeconomic status order, and health across countries. Our analyses showed that subjective SES and health were positively related in all countries under study. The higher one's subjective SES, the better the self-reported health and the greater the psychological wellbeing. This relationship held, even when controlling for a range of objective indicators of SES, namely education, household income, and occupational prestige. Furthermore, analyses revealed sizable cross-national variation in the size of the subjective SES-health association across countries. A crucial fact was that the size of the subjective SEShealth association in a country was largely independent of the income distribution and the overall level of wealth in a country.

\subsubsection{Income comparisons, income inequality, and health}

Chapter 4 focused on a mechanism assumed to link social stratification on the country-level to population health. In a sample of 23 European countries, we found that income inequality is indeed negatively correlated with self-rated health 
and psychological wellbeing, even when accounting for differences in GDP per capita. Given the fact that status competition is one of the most often proposed mechanisms linking income inequality to individual health, we hypothesized that the negative effect of income inequality on health should be strongest for those who attach the highest importance to comparing their income to the incomes of others. While we did find a negative direct relationship of the reported importance of income comparison to health - those who found comparing their incomes to those of others reported worse health and less wellbeing-, this relationship had the same size in all countries, regardless of the level of income inequality in a country. The same was true for different levels of income: The relationship between the reported importance of income comparisons to health had the same size at all levels of individual income.

\subsubsection{The doctor-patient relationship and the social gra- dient in health}

Chapter 5 then returned to the relationship between education and self-rated health and explored the relational aspects of the SES gradient in health by focusing on the doctor-patient relationship. Two different models of the doctorpatient relationship - the paternalistic or doctor-centered one and the egalitarian or patient-centered one - were examined to assess their association with the educational gradient in health. We were able to detect a number of direct associations of some aspects of the doctor-patient relationship with self-rated health. Reports that doctors are easy to understand and treat their patients as equals are associated with better self-reported health. When respondents, however, report that doctors do not tell the whole truth and are not willing to admit mistakes, it goes along with worse self-reported health. Despite these direct effects, the doctorpatient relationship was not found to be a mediator for the educational gradient in health. Even when accounting for any differences in the doctor-patient relationship among the educational groups, the association between education and self-reported health remains the same. What was striking, however, was the fact that some aspects of the doctor-patient relationship had different health effects for the educational groups. Analyses revealed that the positive association between reporting that doctors are easy to understand is solely driven by the lower educated, as for those with medium or high educational degrees, there is virtually no association. Reports that patients are reluctant to ask questions were also found to be unrelated to health for those with medium and high education, but had a strong negative association with health for the lower educated. 


\subsection{Discussion and implications}

The findings reported in this book have several important implications for research on social stratification and health.

Firstly, the substantial variability in the relationship between education and health across countries (as reported in Chapter 2) illustrate the malleability of the social gradient in health. If the relationship between SES and health were solely due to biological or genetic factors, the relationship should be fairly constant across countries and states. The revealed variation, however, underlines the importance of social forces that govern health and wellbeing. Also, the striking similarity between Eastern European countries and some regions of the US raises speculation about the underlying factors causing this resemblance. Rudimentary social protection and problems with the health care systems seem likely candidates.

Secondly, we find a robust association between subjective SES and health in a large number of countries, even over and above the associations of objective SES indicators with health (as reported in Chapter 3). We are also able to detect substantial variation in the size of the subjective SES-health gradient across countries. However, this variation appears to be unrelated to national income and income inequality in a country. The fact that subjective SES is not ameliorated by higher national income could point to that subjective SES is not merely reflecting unmeasured aspects of objective SES, but is indeed an indicator of distinct subjective aspects of SES.

Thirdly, we are able to call into question the mechanism of status differentiation as a driving force of the negative income inequality-health relationship and the social gradient in health. Researchers, such as Wilkinson and Pickett (2010) and Marmot (2004), identified this as an important factor explaining the SEShealth association. Our cross-national study, however, conducted an innovative and crucial test of this mechanism, making use of two important health outcomes and was unable to find support for the status differentiation mechanism. Future research on the income inequality-health correlation should consider focusing on other possible linking mechanisms. For instance, income inequality has been shown to skew politics in favor of the well-off in a society (Hacker and Pierson, 2010), which could also have repercussions on population health by deteriorating welfare provision to those who are worse off.

Fourthly, when comparing the findings across Chapters 2 and 3, the apparent sensitivity of the income inequality-health relationship to the selection of countries under study is revealed. While in Chapter 3 we are unable to detect a negative relationship between income inequality and two health outcomes in a sample of 27 countries from around the world, we find such a relationship in a sample of 
23 European countries in Chapter 4. We were able to cast doubt upon one of the popular mechanisms assumed to underlie the income inequality-health relationship, and this finding calls the existence of a universal relationship between income inequality and health into question (e.g. Beckfield, 2004; Lynch et al., 2004).

Fifthly, we show that creating a more egalitarian doctor-patient relationship in health care can have positive results for lower educated respondents. Although the educational gradient in health is not due to any systematic differences in the doctor-patient relationship that the different educational group experience, we reveal that some aspects of the doctor-patient relationship do have varying effects for the educational groups. Particularly, the lower educated seem to benefit from a more egalitarian doctor-patient relationship: thus, a continued move to a more egalitarian doctor-patient relationship. For instance, this could occur via communication training for physicians and by creating incentive structures in the health care system for more active patients, which might improve the health of the lower educated and, thus, compress the social gradient in health, ultimately contributing to a better health status of the overall population.

\subsection{Contributions}

The selection of studies presented in this book make several distinct contributions. Firstly, we emphasize the importance of not only looking beyond national borders when studying the relationship between SES and health, but also revealing the relevance of looking closely at heterogeneities within countries under study in Chapter 2. This is an important contribution to the literature comprising the comparative turn in health inequality research, which may remind this nascent research field of the fallacy known as "methodological nationalism" (Mau and Verwiebe, 2010; Wimmer and Glick Schiller, 2002).

Secondly, we reveal a robust association of subjective SES and health for the first time in a cross-national setting from around the world in Chapter 3. Furthermore, we are also able to show that this relationship holds in all of the 27 countries under study after controlling for the objective SES markers of income, education, and occupational prestige. While previous studies have largely focused on single countries, and most frequently on the US or the UK, our study is able to show substantial variation in the size of the subjective SES-health association across countries.

Thirdly, we present a novel test of the status competition mechanism that has been hypothesized as linking the relationship between relative income, income inequality, and health in Chapter 4 . In a 23-country study, we were able to show 
that individuals' social comparison orientation with respect to income does not exacerbate the negative health effects of relative income or income inequality in 23 European countries, casting serious doubt on the causal link between income inequality and health.

Fourthly, we explore a novel dimension of the health gradient literature, namely the doctor-patient relationship in Chapter 5. While the relationship between doctors and patients has shifted tremendously in the past decades towards a more egalitarian model of the relation, the consequences for the social gradient in health have so far been left unexplored. Our results allow us to identify important areas where the social gradient in health can potentially be reduced.

\subsection{Limitations and directions for future research}

Being a genuinely cross-national study, the Chapters in this book have to face the limitations that apply to most cross-national research.

A major problem for cross-national research on health inequalities is one of its core aspects, namely the measurement of health. A major methodological step is improving the measurements of health outcomes across countries to be able to assess and remove measurement error and any measurement invariance across countries. For the Chapters in the present book, this was only possible for the psychological wellbeing used measure in Chapter 4 (Van de Velde et al., 2010a), but it can be hoped that cross-national health survey data will soon be able to provide the necessary information for state-of-the-art analytical techniques. A greater focus on multi-item measures of core variables to allow for the assessment of measurement equivalence (Davidov et al., 2014) or the use of anchoring vignettes (King et al., 2004), as it has already been implemented in the Survey of Health, Aging, and Retirement in Europe (SHARE, Börsch-Supan et al., 2013), is greatly desirable.

Furthermore, due to the great reliance of cross-national research on crosssectional data, causal interpretations of the results can easily be called into question. This creates the problem of being unable to distinguish between health selection and social causation on the level of individuals, but also that the findings of country effects might be plagued by omitted variable bias. For improving the understanding of SES effects on health, the collection of longitudinal data on the individual level offers great potential. Again, the SHARE data (Börsch-Supan et al., 2013) will become a valuable resource. For understanding country effects (also in the absence of longitudinal data on the individual level), analyses of panels of country-level data, such as Avendano (2012), Beckfield (2004), or Pop et al. 
(2013), can yield important insights, especially when making use of a fixed-effects framework that allows one to account for any time-constant factors, even when they are unobserved. However, these models are limited in their ability to control for country differences in population composition. In order to overcome this, analyses of pooled cross-sectional cross-national data sets are a promising line of research (Peter et al., 2014).

As already demonstrated in Chapter 2, future research (e.g. Kibele et al., 2014) can potentially yield important insights when properly modeling all the relevant levels in a spatial hierarchy and when contextual-level effects are of interest. The understanding of the consequences of an "omitted level bias" deserve more attention in future research.

The majority of the countries that are studied in cross-national research are what some (Henrich et al., 2010) have called 'WEIRD:' Western, Educated, Industrialized, Rich, and Democratic, which raises the question of how far the findings from our studies can be generalized to a global context. In Chapter 3, we were able to step out of the Western context a bit by including also non-Western countries, but future research should focus on increasing the scope of cross-national research on health inequalities, assessing whether results obtained in a WEIRD context can be replicated under different context conditions. Comparative research on health gradients is an emerging and highly dynamic field that can greatly benefit from drawing on different data sources (e.g Corsi et al., 2012) and, as done by Olafsdottir et al. (2013) and here in Chapter 2, by pooling different data sets. A further standardization of the measures used in studies would greatly benefit such endeavors. 



\section{Bibliography}

Adler, Nancy E., 2013. 'Health Disparities. Taking on the Challenge.' Perspectives on Psychological Science 8(6): 679-681. doi: 10.1177/1745691613506909.

Adler, Nancy E., Thomas Boyce, Margaret A. Chesney, Sheldon Cohen, Susan Folkman, Robert L. Kahn, and S. Leonard Syme, 1994. 'Socioeconomic Status and Health. The Challenge of the Gradient.' American Psychologist 49(1): 15-24. doi: 10.1037/0003-066X.49.1.15.

Adler, Nancy E., Elissa S. Epel, Grace Castellazzo, and Jeannette R. Ickovics, 2000. 'Relationship of Subjective and Objective Social Status With Psychological and Physiological Functioning. Preliminary Data in Healthy White Women.' Health Psychology 19(6): 586-592. doi: 10.1037/0278-6133.19.6.586.

Adler, Nancy E. and Joan M. Ostrove, 1999. 'Socioeconomic Status and Health. What We Know and What We Don't.' Annals of the New York Academy of Sciences 896(1): 3-15. doi: 10.1111/j.1749-6632.1999.tb08101.x.

Adler, Nancy E. and David H. Rehkopf, 2008. 'US Disparities in Health. Descriptions, Causes, and Mechanisms.' Annual Review of Public Health 29: 235-252. doi: 10.1146/annurev.publhealth.29.020907.090852.

Aldabe, Benedicte, Robert Anderson, Maija Lyly-Yrjänäinen, Agnès Parent-Thirion, Greet Vermeylen, Cecily C. Kelleher, and Isabelle Niedhammer, 2011. 'Contribution of Material, Occupational, and Psychosocial Factors in the Explanation of Social Inequalities in Health in 28 Countries in Europe.' Journal of Epidemiology and Community Health 65(12): 1123-1131. doi: $10.1136 /$ jech.2009.102517.

Allison, Paul D., 2002. Missing Data. Thousand Oaks, CA: Sage. doi: 10.4135/9781412985079.

- 2009. Fixed Effects Regression Models. Thousand Oaks, CA: Sage. doi: $10.4135 / 9781412993869$.

Atkinson, Anthony B., 2003. 'Income Inequality in OECD Countries. Data and Explanations.' CESifo Economic Studies 49(4): 479-513. doi: 10.1093/cesifo/49.4.479.

Avendano, Mauricio, 2012. 'Correlation or Causation? Income Inequality and Infant Mortality in Fixed-Effects Models in the Period 1960-2008 in 34 OECD Countries.' Social Science and Medicine 75(4): 754-760. doi: 10.1016/j.socscimed.2012.04.017.

Avendano, Mauricio, M. Maria Glymour, James Banks, and Johan P. Mackenbach, 2009. 'Health Disadvantage in US Adults Aged 50 to 74 Years. A Comparison of the Health of Rich and 
Poor Americans with that of Europeans.' American Journal of Public Health 99(3): 540-548. doi: 10.2105/AJPH.2008.139469.

Avendano, Mauricio and Ichiro Kawachi, 2011. 'The Search for Explanations of the American Health Disadvantage Relative to the English.' American Journal of Epidemiology 173(8): 866-869. doi: 10.1093/aje/kwq484.

_, 2014. 'Why Do Americans Have Shorter Life Expectancy and Worse Health Than Do People in Other High-Income Countries?' Annual Review of Public Health 35: 307-325. doi: 10.1146/annurev-publhealth-032013-182411.

Avendano, Mauricio, Renske Kok, Maria Glymour, Lisa F. Berkman, Ichiro Kawachi, Anton E. Kunst, and Johan P. Mackenbach, 2011. 'Do Americans Have Higher Mortality Than Europeans at All Levels of the Education Distribution? A Comparison of the United States and 14 European Countries.' In Crimmins, Eileen M., Samuel H. Preston, and Barney Cohen, eds., International Differences in Mortality at Older Ages. Dimensions and Sources, pp. 313-332. Washington, DC: National Academies Press. doi: 10.17226/12945.

Bambra, Clare, 2011. 'Health Inequalities and Welfare State Regimes. Theoretical Insights on a Public Health Puzzle.' Journal of Epidemiology and Community Health 65(9): 740-745. doi: 10.1136/jech.2011.136333.

Banks, James, Michael Marmot, Zoe Oldfield, and James P. Smith, 2006. 'Disease and Disadvantage in the United States and in England.' JAMA 295(17): 2037-2045. doi: 10.1001/jama.295.17.2037.

— 2009. 'The SES-Health Gradient on Both Sides of the Atlantic.' In Wise, David A., ed., Developments in the Economics of Aging, pp. 359-406. Chicago, IL: University of Chicago Press. doi: 10.7208/chicago/9780226903361.003.0011.

Banks, James, Alastair Muriel, and James P. Smith, 2010. 'Disease Prevalence, Disease Incidence, and Mortality in the United States and in England.' Demography 47(Supplement): S211-S231. doi: $10.1353 /$ dem.2010.0008.

Bardage, Carola, Saskia M. F. Pluijm, Nancy L. Pedersen, Dorly J. H. Deeg, Marja Jylhä, Marianna Noale, Tzvia Blumstein, and Ángel Otero, 2005. 'Self-Rated Health Among Older Adults. A Cross-National Comparison.' European Journal of Ageing 2(2): 149-158. doi: 10.1007/s10433-005-0032-7.

Barry, Christine A., Colin P. Bradley, Nicky Britten, Fiona A. Stevenson, and Nick Barber, 2000. 'Patients' Unvoiced Agendas in General Practice Consultations. Qualitative Study.' BMJ 320(7244): 1246-1250. doi: 10.1136/bmj.320.7244.1246.

Bayer, Ronald, Amy L. Fairchild, Kim Hopper, and Constance A. Nathanson, 2013. 'Confronting the Sorry State of US Health.' Science 341(6149): 962-963. doi: 10.1126/science.1241249.

Beck, Ulrich, 1992. Risk Society. Towards a New Modernity. London: Sage.

Beckfield, Jason, 2004. 'Does Income Inequality Harm Health? New Cross-National Evidence.' Journal of Health and Social Behavior 45(3): 231-248. doi: 10.1177/002214650404500301. 
Beckfield, Jason and Nancy Krieger, 2009. 'Epi + Demos + Cracy. Linking Political Systems and Priorities to the Magnitude of Health Inequities. Evidence, Gaps, and a Research Agenda.' Epidemiologic Reviews 31(1): 152-177. doi: 10.1093/epirev/mxp002.

Beckfield, Jason, Sigrun Olafsdottir, and Elyas Bakhtiari, 2013. 'Health Inequalities in Global Context.' American Behavioral Scientist 57(8): 1014-1039. doi: 10.1177/0002764213487343.

Beham, Barbara, Sonja Drobnič, and Patrick Präg, 2011. 'Work Demands and Resources and the Work-Family Interface. Testing a Salience Model on German Service Sector Employees.' Journal of Vocational Behavior 78(1): 110-122. doi: 10.1016/j.jvb.2010.07.008.

_ 2014. 'The Work-Family Interface of Service Sector Workers. A Comparison of Work Resources and Professional Status across Five European Countries.' Applied Psychology 63(1): 29-61. doi: 10.1111/apps.12012.

Beham, Barbara, Patrick Präg, and Sonja Drobnic, 2012. 'Who's Got the Balance? A Study of Satisfaction with the Work-Family Balance among Part-Time Service Sector Employees in Five Western European Countries.' International Journal of Human Resource Management 23(18): 3725-3741. doi: 10.1080/09585192.2012.654808.

Bell, Bethany A., Grant B. Morgan, Jason A. Schoeneberger, Jeffrey D. Kromrey, and John M. Ferron, 2014. 'How Low Can You Go? An Investigation of the Influence of Sample Size and Model Complexity on Point and Interval Estimates in Two-Level Linear Models.' Methodology 10(1): 1-11. doi: 10.1027/1614-2241/a000062.

Bengtsson, Tommy and Frans Van Poppel, 2011. 'Socioeconomic Inequalities in Death from Past to Present. An Introduction.' Explorations in Economic History 48(3): 343-356. doi: 10.1016/j.eeh.2011.05.004.

Berkman, Lisa and Arnold M. Epstein, 2008. 'Beyond Health Care. Socioeconomic Status and Health.' New England Journal of Medicine 358(23): 2509-2510. doi: 10.1056/NEJMe0802773.

Berkman, Lisa F. and S. Leonard Syme, 1979. 'Social Networks, Host Resistance, and Mortality. A Nine-Year Follow-Up Study of Alameda County Residents.' American Journal of Epidemiology 109(2): 186-204. doi: 10.1093/oxfordjournals.aje.a112674.

Bezruchka, Stephen, 2012. 'The Hurrider I Go the Behinder I Get. The Deteriorating International Ranking of US Health Status.' Annual Review of Public Health 33: 157-173. doi: 10.1146/annurev-publhealth-031811-124649.

Bloom, Samuel W., 2002. The Word as Scalpel. A History of Medical Sociology. New York: Oxford University Press.

Braveman, Paula, Susan Egerter, and David R. Williams, 2011. 'The Social Determinants of Health. Coming of Age.' Annual Review of Public Health 32: 381-398. doi: 10.1146/annurevpublhealth-031210-101218.

Braveman, Paula A., Catherine Cubbin, Susan Egerter, Sekai Chideya, Kristen S. Marchi, Marilyn Metzler, and Samuel Posner, 2005. 'Socioeconomic Status in Health Research. One Size Does Not Fit All.' JAMA 294(22): 2879-2888. doi: 10.1001/jama.294.22.2879. 
Brennan, Geoffrey and Philip Pettit, 2004. The Economy of Esteem. An Essay on Civil and Political Society. New York: Oxford University Press. doi: 10.1093/0199246483.001.0001/acprof9780199246489 .

Brennenstuhl, Sarah, Amélie Quesnel-Vallée, and Peggy McDonough, 2012. 'Welfare Regimes, Population Health, and Health Inequalities. A Research Synthesis.' Journal of Epidemiology and Community Health 66(5): 397-409. doi: 10.1136/jech-2011-200277.

Börsch-Supan, Axel, Martina Brandt, Christian Hunkler, Thorsten Kneip, Julie Korbmacher, Frederic Malter, Barbara Schaan, Stephanie Stuck, and Sabrina Zuber, 2013. 'Data Resource Profile. The Survey of Health, Aging, and Retirement in Europe (SHARE).' International Journal of Epidemiology 42(4): 992-1001. doi: 10.1093/ije/dyt088.

Bryan, Mark L. and Stephen P. Jenkins, 2015. 'Multilevel Modeling of Country Effects. A Cautionary Tale.' European Sociological Review doi: 10.1093/esr/jcv059.

Bultman, Dara C. and Bonnie L. Svarstad, 2000. 'Effects of Physician Communication Style on Client Medication Beliefs and Adherence with Antidepressant Treatment.' Patient Education and Counseling 40(2): 173-185. doi: 10.1016/S0738-3991(99)00083-X.

Burström, Bo and Peeter Fredlund, 2001. 'Self-Rated Health. Is It as Good a Predictor of Subsequent Mortality Among Adults in Lower as Well as in Higher Social Classes?' Journal of Epidemiology and Community Health 55(11): 836-840. doi: 10.1136/jech.55.11.836.

Buunk, Abraham P. and Frederick X. Gibbons, 1997. Health, Coping, and Well-Being. Perspectives from Social Comparison Theory. Mahwah, NJ: Lawrence Erlbaum.

—, 2007. 'Social Comparison. The End of a Theory and the Emergence of a Field.' Organizational Behavior and Human Decision Processes 102(1): 3-21. doi: 10.1016/j.obhdp.2006.09.007.

Byrne, Patrick S. and Barrie E. L. Long, 1976. Doctors Talking to Patients. A Study of the Verbal Behaviour of General Practitioners Consulting in Their Surgeries. London: HMSO.

CDC, 2008. Behavioral Risk Factor Surveillance System (BRFSS) Survey Data. Atlanta, GA: US Department of Health and Human Services, Centers for Disease Control and Prevention.

Chan, Tak Wing and John H. Goldthorpe, 2007. 'Class and Status. The Conceptual Distinction and its Empirical Relevance.' American Sociological Review 72(4): 512-532. doi: 10.1177/000312240707200402.

Chandola, Tarani, 1998. 'Social Inequality in Coronary Heart Disease. A Comparison of Occupational Classifications.' Social Science and Medicine 47(4): 525-533. doi: 10.1016/S02779536(98)00141-5.

Chandola, Tarani, Mel Bartley, Amanda Sacker, Crispin Jenkinson, and Michael Marmot, 2003. 'Health Selection in the Whitehall II Study, UK.' Social Science and Medicine 56(10): 20592072. doi: 10.1016/S0277-9536(02)00201-0.

Charles, Cathy, Amiram Gafni, and Tim Whelan, 1997. 'Shared Decision-Making in the Medical Encounter. What Does It Mean? (Or, It Takes at Least Two to Tango).' Social Science and Medicine 44(5): 681-692. doi: 10.1016/S0277-9536(96)00221-3. 
Charmaz, Kathy and Dana Rosenfeld, 2010. 'Chronic Illness.' In Cockerham, William C., ed., The New Blackwell Companion to Medical Sociology, pp. 312-333. Malden, MA: Wiley-Blackwell. doi: $10.1002 / 9781444314786 . c h 14$.

Chen, Jarvis T., Jason Beckfield, Pamela D. Waterman, and Nancy Krieger, 2013. 'Can Changes in the Distributions of and Associations Between Education and Income Bias Temporal Comparisons of Health Disparities? An Exploration With Causal Graphs and Simulations.' American Journal of Epidemiology 177(9): 870-881. doi: 10.1093/aje/kwt041.

Cialdini, Robert B. and Vladas Griskevicius, 2010. 'Social Influence.' In Baumeister, Roy F. and Eli J. Finkel, eds., Advanced Social Psychology. The State of the Science, pp. 385-417. New York: Oxford University Press.

Clark, Andrew E., Paul Frijters, and Michael A. Shields, 2008. 'Relative Income, Happiness, and Utility. An Explanation for the Easterlin Paradox and Other Puzzles.' Journal of Economic Literature 46(1): 95-144. doi: 10.1257/jel.46.1.95.

Clark, Andrew E. and Claudia Senik, 2010. 'Who Compares to Whom? The Anatomy of Income Comparisons in Europe.' The Economic Journal 120(544): 573-594. doi: 10.1111/j.14680297.2010.02359.x.

Cohen, Jacob, Patricia Cohen, Stephen G. West, and Leona S. Aiken, 2003. Applied Multiple Regression/Correlation Analysis for the Behavioral Sciences. Mahwah, NJ: Erlbaum, 3rd edition. doi: 10.4324/9780203774441.

Cohen, Sheldon, Cuneyt M. Alper, William J. Doyle, Nancy E. Adler, John J. Treanor, and Ronald B. Turner, 2008. 'Objective and Subjective Socioeconomic Status and Susceptibility to the Common Cold.' Health Psychology 27(2): 268-274. doi: 10.1037/0278-6133.27.2.268.

Coleman, James, Elihu Katz, and Herbert Menzel, 1957. 'The Diffusion of an Innovation Among Physicians.' Sociometry 20(4): 253-270. doi: 10.2307/2785979.

Collins, Amy Love and Noreen Goldman, 2008. 'Perceived Social Position and Health in Older Adults in Taiwan.' Social Science and Medicine 66(3): 536-544. doi: 10.1016/j.socscimed.2007.10.004.

Corsi, Daniel J., Melissa Neuman, Jocelyn E. Finlay, and SV Subramanian, 2012. 'Demographic and Health Surveys. A Profile.' International Journal of Epidemiology 41(6): 1602-1613. doi: $10.1093 / \mathrm{ije} /$ dys 184 .

Crimmins, Eileen M., Samuel H. Preston, and Barney Cohen, 2011. International Differences in Mortality at Older Ages. Dimensions and Sources. Washington, DC: National Academies Press. doi: 10.17226/12945.

Crow, R., H. Gage, S. Hampson, J. Hart, A. Kimber, L. Storey, and H. Thomas, 2003. 'The Measurement of Satisfaction with Healthcare. Implications for Practice from a Systematic Review of the Literature.' Health Technology Assessment 32(6): 1-244. doi: 10.3310/hta6320.

Cundiff, Jenny M., Timothy W. Smith, Bert N. Uchino, and Cynthia A. Berg, 2013. 'Subjective Social Status. Construct Validity and Associations with Psychosocial Vulnerability and Self-Rated Health.' International Journal of Behavioral Medicine 20(1): 148-158. doi: $10.1007 / \mathrm{s} 12529-011-9206-1$. 
Dahlin, Johanna and Juho Härkönen, 2013. 'Cross-National Differences in the Gender Gap in Subjective Health in Europe. Does Country-Level Gender Equality Matter?' Social Science and Medicine 98: 24-28. doi: 10.1016/j.socscimed.2013.08.028.

Daniels, Norman, 2008. Just Health. Meeting Health Needs Fairly. New York: Cambridge University Press. doi: 10.1017/CBO9780511809514.

Davey Smith, George, 1996. 'Income Inequality and Mortality. Why Are They Related?' BMJ 312(7037): 987-988. doi: 10.1136/bmj.312.7037.987.

Davidov, Eldad, Bart Meuleman, Jan Cieciuch, Peter Schmidt, and Jaak Billiet, 2014. 'Measurement Equivalence in Cross-National Research.' Annual Review of Sociology 40: 55-75. doi: 10.1146/annurev-soc-071913-043137.

De Bruin, Arnout, H. Susan J. Picavet, and A. Nossikov, 1996. Health Interview Surveys. Towards International Harmonization of Methods and Instruments. Copenhagen: WHO Regional Publications.

Deaton, Angus, 2013. The Great Escape. Health, Wealth, and the Origins of Inequality. Princeton, NJ: Princeton University Press. doi: 10.2307/j.ctt3fgxbm.

Deci, Edward L., Richard Koestner, and Richard M. Ryan, 1999. 'A Meta-Analytic Review of Experiments Examining the Effects of Extrinsic Rewards on Intrinsic Motivation.' Psychological Bulletin 125(6): 627-668. doi: 10.1037/0033-2909.125.6.627.

Demakakos, Panayotes, James Nazroo, Elizabeth Breeze, and Michael Marmot, 2008. 'Socioeconomic Status and Health. The Role of Subjective Social Status.' Social Science and Medicine 67(2): 330-340. doi: 10.1016/j.socscimed.2008.03.038.

Department of Health, 2001. The Expert Patient. A New Approach to Chronic Disease Management for the 21st Century. London: HMSO.

Dickerson, Sally and Margaret E. Kemeny, 2004. 'Acute Stressors and Cortisol Responses. A Theoretical Integration and Synthesis of Laboratory Research.' Psychological Bulletin 130(3): 355-391. doi: 10.1037/0033-2909.130.3.355.

Djundeva, Maja, Melinda Mills, Rafael Wittek, and Nardi Steverink, 2015. 'Receiving Instrumental Support in Late Parent-Child Relationships and Parental Depression.' Journals of Gerontology Series B: Psychological Sciences and Social Sciences 70(6): 981-994. doi: 10.1093 /geronb/gbu136.

Dow, William H. and David H. Rehkopf, 2010. 'Socioeconomic Gradients in Health in International and Historical Context.' Annals of the New York Academy of Sciences 1186(1): 24-36. doi: 10.1111/j.1749-6632.2009.05384.x.

Drobnič, Sonja, Barbara Beham, and Patrick Präg, 2010. 'Good Job, Good Life? Working Conditions and Quality of Life in Europe' Social Indicators Research 99(2): 205-225. doi: $10.1007 / \mathrm{s} 11205-010-9586-7$.

Dunn, James R., Gerry Veenstra, and Nancy Ross, 2006. 'Psychosocial and Neo-Material Dimensions of SES and Health Revisited. Predictors of Self-Rated Health in a Canadian National Survey.' Social Science and Medicine 62(6): 1465-1473. doi: 10.1016/j.socscimed.2005.07.038. 
Durkheim, Emile, 1933. The Division of Labor in Society. Glencoe, IL: Free Press.

— 1951. Suicide. New York: Free Press.

Eikemo, Terje Andreas, Martijn Huisman, Clare Bambra, and Anton E. Kunst, 2008a. 'Health Inequalities According to Educational Level in Different Welfare Regimes. A Comparison of 23 European Countries.' Sociology of Health and Illness 30(4): 565-582. doi: 10.1111/j.14679566.2007.01073.x.

Eikemo, Terje Andreas, Anton E. Kunst, Ken Judge, and Johan P. Mackenbach, 2008b. 'ClassRelated Health Inequalities Are Not Larger in the East. A Comparison of Four European Regions Using the New European Socio-Economic Classification.' Journal of Epidemiology and Community Health 62(12): 1072-1078. doi: 10.1136/jech.2007.072470.

Ellwardt, Lea, Sascha Peter, Patrick Präg, and Nardi Steverink, 2014. 'Social Contacts of Older People in 27 European Countries. The Role of Welfare Spending and Economic Inequality.' European Sociological Review 30(4): 413-430. doi: 10.1093/esr/jcu046.

Elo, Irma T., 2009. 'Social Class Differentials in Health and Mortality. Patterns and Explanations in Comparative Perspective.' Annual Review of Sociology 35: 553-572. doi: 10.1146/annurevsoc-070308-115929.

Elstad, Jon Ivar, 2004. 'Health and Status Attainment. Effects of Health on Occupational Achievement among Employed Norwegian Men.' Acta Sociologica 47(2): 127-140. doi: $10.1177 / 0001699304043824$.

Enders, Craig K. and Davood Tofighi, 2007. 'Centering Predictor Variables in Cross-Sectional Multilevel Models. A New Look at an Old Issue.' Psychological Methods 12(2): 121-138. doi: 10.1037/1082-989x.12.2.121.

Epel, Elissa S., Elizabeth H. Blackburn, Jue Lin, Firdaus S. Dhabhar, Nancy E. Adler, Jason D. Morrow, and Richard M. Cawthon, 2004. 'Accelerated Telomere Shortening in Response to Life Stress.' Proceedings of the National Academy of Sciences 101(49): 17312-17315. doi: 10.1073/pnas.0407162101.

Erikson, Robert and John H. Goldthorpe, 1992. The Constant Flux. A Study of Class Mobility in Industrial Societies. Oxford: Clarendon.

Eurostat, 2011. Statistics Database. Economy and Finance. Luxembourg: europa.eu/portal/page/portal/statistics/search_database.

Euteneuer, Frank, 2014. 'Subjective Social Status and Health.' Current Opinion in Psychiatry 27(5): 337-343. doi: 10.1097/YCO.0000000000000083.

Evans, Mariah D.R. and Jonathan Kelley, 2004. 'Subjective Social Location. Data From 21 Nations.' International Journal of Public Opinion Research 16(1): 3-38. doi: 10.1093/ijpor/16.1.3.

EVS, 2011. European Values Study 2008 (4th wave). Integrated dataset (Vers. 3.0.0). Cologne: GESIS Data Archive Study no. ZA4800. doi: 10.4232/1.11004. 
EVS and GESIS, 2011. EVS 2008 Method Report. Documentation of the Full Data Release 30/11010. Related to the Integrated Dataset, Archive Study No. ZA4800. Cologne: GESIS. doi: $10.4232 / 1.10188$.

Ezzati, Majid, Ari B. Friedman, Sandeep C. Kulkarni, and Christopher J. L. Murray, 2008. 'The Reversal of Fortunes. Trends in County Mortality and Cross-County Mortality Disparities in the United States.' PLoS Medicine 5(4): e66. doi: 10.1371/journal.pmed.0050066.

Festinger, Leon, 1954. 'A Theory of Social Comparison Processes.' Human Relations 7(2): 117-140. doi: 10.1177/001872675400700202.

Fiske, Susan T., 2011. Envy Up, Scorn Down. How Status Divides Us. New York: Russell Sage.

Frank, Robert H., 1985. Choosing the Right Pond. Human Behavior and the Quest for Status. New York: Oxford University Press.

Frieswijk, Nynke, Abraham P. Buunk, Nardi Steverink, and Jordis P. J. Slaets, 2007. 'Subjective Well-Being in Frail Older Persons. Why Social Comparison Orientation and Self-Management Are Important.' Revue Internationale de Psychologie Sociale 20(1): 105-124.

Fuchs, Susanne and Claus Offe, 2009. 'Welfare State Formation in the Enlarged European Union. Patterns of Reform in Postcommunist States.' In Rumford, Chris, ed., The Sage Handbook of European Studies, pp. 420-441. Thousand Oaks, CA: Sage. doi: 10.4135/9780857021045.n24.

Furnée, Carina A., Wim Groot, and Henriëtte Maassen van den Brink, 2008. 'The Health Effects of Education. A Meta-Analysis.' European Journal of Public Health 18(4): 417-421. doi: 10.1093/eurpub/ckn028.

Galobardes, Bruna, Mary Shaw, Debbie A. Lawlor, John W. Lynch, and George Davey Smith, 2006. 'Indicators of Socioeconomic Position (Part 1).' Journal of Epidemiology and Community Health 60(1): 7-12. doi: 10.1136/jech.2004.023531.

Ganzeboom, Harry B. G., Paul M. De Graaf, and Donald J. Treiman, 1992. 'A Standard International Socio-Economic Index of Occupational Status.' Social Science Research 21(1): 1-56. doi: 10.1016/0049-089X(92)90017-B.

Garbarski, Dana, 2010. 'Perceived Social Position and Health. Is there a Reciprocal Relationship?' Social Science and Medicine 70(5): 692-699. doi: 10.1016/j.socscimed.2009.11.007.

Gelfand, Michele J., Jana L. Raver, Lisa Nishii, Lisa M. Leslie, Janetta Lun, Beng Chong Lim, Lili Duan, Assaf Almaliach, Soon Ang, Jakobina Arnadottir, Zeynep Aycan, Klaus Boehnke, Pawel Boski, Rosa Cabecinhas, Darius Chan, Jagdeep Chhokar, Alessia D’Amato, Montse Ferrer, Iris C. Fischlmayr, Ronald Fischer, Marta Fülöp, James Georgas, Emiko S. Kashima, Yoshishima Kashima, Kibum Kim, Alain Lempereur, Patricia Marquez, Rozhan Othman, Bert Overlaet, Penny Panagiotopoulou, Karl Peltzer, Lorena R. Perez-Florizno, Larisa Ponomarenko, Anu Realo, Vidar Schei, Manfred Schmitt, Peter B. Smith, Nazar Soomro, Erna Szabo, Nalinee Taveesin, Midori Toyama, Evert Van de Vliert, Naharika Vohra, Colleen Ward, and Susumu Yamaguchi, 2011. 'Differences Between Tight and Loose Cultures. A 33-Nation Study.' Science 332(6033): 1100-1104. doi: 10.1126/science.1197754. 
Gesthuizen, Maurice, Tim Huijts, and Gerbert Kraaykamp, 2012. 'Explaining Health Marginalization of the Lower Educated. The Role of Cross-National Variations in Health Expenditure and Labour Market Conditions.' Sociology of Health and Illness 34(4): 591-607. doi: 10.1111/j.1467-9566.2011.01390.x.

Geyer, Siegfried, Örjan Hemström, Richard Peter, and Denny Vågerö, 2006. 'Education, Income, and Occupational Class Cannot Be Used Interchangeably in Social Epidemiology. Empirical Evidence Against a Common Practice.' Journal of Epidemiology and Community Health 60(9): 804-810. doi: 10.1136/jech.2005.041319.

Gilbert, Paul, John Price, and Steven Allan, 1995. 'Social Comparison, Social Attractiveness, and Evolution. How Might They Be Related?' New Ideas in Psychology 13(2): 149-165. doi: 10.1016/0732-118X(95)00002-X.

Goldthorpe, John H., 2010. 'Analyzing Social Inequality. A Critique of Two Recent Contributions from Economics and Epidemiology.' European Sociological Review 26(6): 731-744. doi: $10.1093 /$ esr/jcp046.

Goodman, Elizabeth, Nancy E. Adler, Stephen R. Daniels, John A. Morrison, Gail B. Slap, and Lawrence M. Dolan, 2003. 'Impact of Objective and Subjective Social Status on Obesity in a Biracial Cohort of Adolescents.' Obesity Research 11(8): 1018-1026. doi: 10.1038/oby.2003.140.

Goodman, Elizabeth, Nancy E. Adler, Ichiro Kawachi, A. Lindsay Frazier, Bin Huang, and Graham A. Colditz, 2001. 'Adolescents' Perceptions of Social Status. Development and Evaluation of a New Indicator.' Pediatrics 108(2): e31. doi: 10.1542/peds.108.2.e31.

Gravelle, Hugh, 1998. 'How Much of the Relationship between Population Mortality and Unequal Distribution of Income is a Statistical Artifact?' BMJ 316(7128): 382-385. doi: 10.1136/bmj.316.7128.382.

Griffin, Simon J., Ann-Louise Kinmonth, Marijcke W. M. Veltman, Susan Gillard, Julie Grant, and Moira Stewart, 2004. 'Effect on Health-Related Outcomes of Interventions to Alter the Interaction Between Patients and Practitioners. A Systematic Review of Trials.' Annals of Family Medicine 2(6): 595-608. doi: 10.1370/afm.142.

Hacker, Jacob S., 2006. The Great Risk Shift. The Assault on American Jobs, Families, Health Care, and Retirement And How You Can Fight Back. New York: Oxford University Press.

Hacker, Jacob S. and Paul Pierson, 2010. Winner-Take-All Politics. How Washington Made the Rich Richer-And Turned Its Back on the Middle Class. New York: Simon and Schuster.

Halaby, Charles N., 2004. 'Panel Models in Sociological Research. Theory into Practice.' Annual Review of Sociology 30: 507-544. doi: 10.1146/annurev.soc.30.012703.110629.

Harper, Sam, Nicholas B. King, Stephen C. Meersman, Marsha E. Reichman, Nancy Breen, and John Lynch, 2010. 'Implicit Value Judgments in the Measurement of Health Inequalities.' Milbank Quarterly 88(1): 4-29. doi: 10.1111/j.1468-0009.2010.00587.x. 
Harrington, Jesse R. and Michele J. Gelfand, 2014. 'Tightness-Looseness across the 50 United States.' Proceedings of the National Academy of Sciences 111(22): 7990-7995. doi: $10.1073 /$ pnas. 1317937111.

Harris, Bernard, 2004. 'Public Health, Nutrition, and the Decline of Mortality. The McKeown Thesis Revisited.' Social History of Medicine 17(3): 379-407. doi: 10.1093/shm/17.3.379.

Hedström, Peter and Richard Swedberg, 1996. 'Social Mechanisms.' Acta Sociologica 39(3): 281-308. doi: 10.1177/000169939603900302.

Henderson, Lawrence Joseph, 1935. 'Physician and Patient as a Social System.' New England Journal of Medicine 212(18): 819-823. doi: 10.1056/NEJM193505022121803.

Henrich, Joseph, Steven J. Heine, and Ara Norenzayan, 2010. 'The Weirdest People in the World?' Behavioral and Brain Sciences 33(2-3): 61-83. doi: 10.1017/S0140525X0999152X.

Heritage, John and Douglas W. Maynard, 2006. 'Problems and Prospects in the Study of Physician-Patient Interaction. Thirty Years of Research.' Annual Review of Sociology 32: 351-374. doi: 10.1146/annurev.soc.32.082905.093959.

_, 2011. 'After 30 Years, Problems and Prospects in the Study of Doctor-Patient Interaction.' In Pescosolido, Bernice A., Jack K. Martin, Jane D. McLeod, and Anne Rogers, eds., Handbook of the Sociology of Health, Illness, and Healing. A Blueprint for the 21 ${ }^{\text {st }}$ Century, pp. 323-342. New York: Springer. doi: 10.1007/978-1-4419-7261-3_17.

Hildebrand, Vincent and Philippe Van Kerm, 2009. 'Income Inequality and Self-Rated Health Status. Evidence from the European Community Household Panel.' Demography 46(4): 805825. doi: 10.1353/dem.0.0071.

Hofstede, Geert, 2001. Culture's Consequences. Comparing Values, Behaviors, Institutions, and Organizations across Nations. Thousand Oaks, CA: Sage, 2nd edition.

Holt-Lunstad, Julianne, Timothy B. Smith, and J. Bradley Layton, 2010. 'Social Relationships and Mortality Risk. A Meta-Analytic Review.' PLoS Medicine 7(7): e1000316. doi: 10.1371/journal.pmed.1000316.

House, James S., 2002. 'Understanding Social Factors and Inequalities in Health. 20 ${ }^{\text {th }}$ Century Progress and $21^{\text {st }}$ Century Prospects.' Journal of Health and Social Behavior 43(2): 125-142. doi: $10.2307 / 3090192$.

Huguet, Pascal, Florence Dumas, Herbert Marsh, Isabelle Régner, Ladd Wheeler, Jerry Suls, Marjorie Seaton, and John Nezlek, 2009. 'Clarifying the Role of Social Comparison in the Big-Fish-Little-Pond Effect (BFLPE). An Integrative Study.' Journal of Personality and Social Psychology 97(1): 156-170. doi: 10.1037/a0015558.

Huijts, Tim and Terje Andreas Eikemo, 2009. 'Causality, Social Selectivity, or Artefacts? Why Socioeconomic Inequalities in Health Are Not Smallest in the Nordic Countries.' European Journal of Public Health 19(5): 452-453. doi: 10.1093/eurpub/ckp103.

Huijts, Tim, Terje Andreas Eikemo, and Vera Skalická, 2010a. 'Income-Related Health Inequalities in the Nordic Countries. Examining the Role of Education, Occupational Class, and Age.' Social Science and Medicine 71(11): 1964-1972. doi: 10.1016/j.socscimed.2010.09.021. 
Huijts, Tim and Gerbert Kraaykamp, 2011. 'Religious Involvement, Religious Context, and Self-Assessed Health in Europe.' Journal of Health and Social Behavior 52(1): 91-106. doi: $10.1177 / 0022146510394950$.

, 2012. 'Formal and Informal Social Capital and Self-Rated Health in Europe. A New Test of Accumulation and Compensation Mechanisms Using a Multi-Level Perspective.' Acta Sociologica 55(2): 143-158. doi: 10.1177/0001699312439080.

Huijts, Tim, Gerbert Kraaykamp, and SV Subramanian, 2013. 'Childlessness and Psychological Well-Being in Context. A Multilevel Study on 24 European Countries.' European Sociological Review 29(1): 32-47. doi: 10.1093/esr/jcr037.

Huijts, Tim, Christiaan W. S. Monden, and Gerbert Kraaykamp, 2010b. 'Education, Educational Heterogamy, and Self-Assessed Health in Europe. A Multilevel Study of Spousal Effects in 29 European Countries.' European Sociological Review 26(3): 261-276. doi: 10.1093/esr/jcp019.

Huppert, Felicia A., Nic Marks, Andrew E. Clark, Johannes Siegrist, Alois Stutzer, Joar Vitters $\varnothing$, and Morten Wahrendorf, 2009. 'Measuring Wellbeing across Europe. Description of the ESS Wellbeing Module and Preliminary Findings.' Social Indicators Research 91(3): 301-315. doi: 10.1007/s11205-008-9346-0.

Idler, Ellen L. and Yael Benyamini, 1997. 'Self-Rated Health and Mortality. A Review of Twenty-Seven Community Studies.' Journal of Health and Social Behavior 38(1): 21-37. doi: $10.2307 / 2955359$.

Idler, Ellen L., Shawna V. Hudson, and Howard Leventhal, 1999. 'The Meanings of Self-Ratings of Health. A Qualitative and Quantitative Approach.' Research on Aging 21(3): 458-476. doi: $10.1177 / 0164027599213006$.

IMF, 2012. World Economic Outlook Database April 2012 Edition. Washington, DC: International Monetary Fund.

ISSP Research Group, 2013. International Social Survey Programme 2011 Health and Health Care. ZA5800 v2.0.0. Cologne: GESIS Data Archive. doi: 10.4232/1.11759.

Jackman, Mary R. and Robert W. Jackman, 1973. 'An Interpretation of the Relation Between Objective and Subjective Social Status.' American Sociological Review 38(5): 569-582. doi: $10.2307 / 2094408$.

Jackson, Elton F., 1962. 'Status Consistency and Symptoms of Stress.' American Sociological Review 27(4): 469-480. doi: 10.2307/2090028.

Jones, Kelvyn and Nina Bullen, 1994. 'Contextual Models of Urban House Prices. A Comparison of Fixed- and Random-Coefficient Models Developed by Expansion.' Economic Geography 70(3): pp. 252-272. doi: 10.2307/143993.

Jowell, Roger, Caroline Roberts, Rory Fitzgerald, and Gillian Eva, 2007. Measuring Attitudes Cross-Nationally. Lessons from the European Social Survey. Los Angeles, CA: Sage. doi: 10.4135/9781849209458. 
Jürges, Hendrik, Mauricio Avendano, and Johan Mackenbach, 2008. 'Are Different Measures of Self-Rated Health Comparable? An Assessment in Five European Countries.' European Journal of Epidemiology 23(12): 773-781. doi: 10.1007/s10654-008-9287-6.

Judge, Ken, Jo-Ann Mulligan, and Michaela Benzeval, 1998. 'Income Inequality and Population Health.' Social Science and Medicine 46(4-5): 567-579. doi: 10.1016/S0277-9536(97)00204-9.

Judson, Timothy J., Allan S. Detsky, and Matthew J. Press, 2013. 'Encouraging Patients to Ask Questions. How to Overcome "White-Coat Silence".' JAMA 309(22): 2325-2326. doi: 10.1001/jama.2013.5797.

Jürges, Hendrik, 2009. 'Healthy Minds in Healthy Bodies. An International Comparison of Education-Related Inequality in Physical Health Among Older Adults.' Scottish Journal of Political Economy 56(3): 296-320. doi: 10.1111/j.1467-9485.2009.00485.x.

Kaba, R. and Prasanna Sooriakumaran, 2007. 'The Evolution of the Doctor-Patient Relationship.' International Journal of Surgery 5(1): 57-65. doi: 10.1016/j.ijsu.2006.01.005.

Kaplan, Mark S., Nathalie Huguet, David H. Feeny, and Bentson H. McFarland, 2010. 'SelfReported Hypertension Prevalence and Income among Older Adults in Canada and the United States.' Social Science and Medicine 70(6): 844-849. doi: 10.1016/j.socscimed.2009.11.019.

Karvonen, Sakari and Ossi Rahkonen, 2011. 'Subjective Social Status and Health in Young People.' Sociology of Health and Illness 33(3): 372-383. doi: 10.1111/j.1467-9566.2010.01285.x.

Kasser, Tim and Richard M. Ryan, 1993. 'A Dark Side of the American Dream. Correlates of Financial Success as a Central Life Aspiration.' Journal of Personality and Social Psychology 65(2): 410-422. doi: 10.1037/0022-3514.65.2.410.

Kedar, Orit and W. Phillips Shively, 2005. 'Introduction to the Special Issue on Multilevel Modeling for Large Clusters.' Political Analysis 13(4): 297-300. doi: 10.1093/pan/mpi027.

Kibele, Eva, 2012. Regional Mortality Differences in Germany. Dordrecht: Springer. doi: 10.1007/978-94-007-4432-5.

Kibele, Eva, Patrick Präg, and Paul Norman, 2014. 'Associations between Area-Level Conditions, Socioeconomic Conditions, and Mortality. Do They Vary over the Life Course?' Paper presented at PAA Annual Meeting in Boston, MA, the RC 28 Spring Meeting in Budapest, and the EPC in Budapest.

Kindig, David A. and Erika R. Cheng, 2013. 'Even as Mortality Fell in Most US Counties, Female Mortality Nonetheless Rose in 42.8 \% of Counties from 1992 to 2006.' Health Affairs 32(3): 451-458. doi: 10.1377/hlthaff.2011.0892.

King, Gary, Christopher J. L. Murray, Joshua A. Salomon, and Ajay Tandon, 2004. 'Enhancing the Validity and Cross-Cultural Comparability of Measurement in Survey Research.' American Political Science Review 98(1): 191-207. doi: 10.1017/S000305540400108X.

King, Nicholas B., Sam Harper, and Meredith E. Young, 2012. 'Use of Relative and Absolute Effect Measures in Reporting Health Inequalities. Structured Review.' BMJ 345(7878): 17. doi: $10.1136 /$ bmj.e5774. 
Kitagawa, Evelyn Mae and Philip Morris Hauser, 1973. Differential Mortality in the United States. A Study in Socioeconomic Epidemiology. Cambridge, MA: Harvard University Press.

Koball, Heather L., Emily Moiduddin, Jamila Henderson, Brian Goesling, and Melanie Besculides, 2010. 'What Do We Know About the Link Between Marriage and Health?' Journal of Family Issues 31(8): 1019-1040. doi: 10.1177/0192513X10365834.

Kohn, Melvin L., 1987. 'Cross-National Research as an Analytic Strategy.' American Sociological Review 52(6): 713-731. doi: 10.2307/2095831.

Kondo, Naoki, Grace Sembajwe, Ichiro Kawachi, Rob M. van Dam, SV Subramanian, and Zentaro Yamagata, 2009. 'Income Inequality, Mortality, and Self-Rated Health. Meta-Analysis of Multilevel Studies.' BMJ 339(7731): 1178-1181. doi: 10.1136/bmj.b4471.

Kooiker, Sjoerd, Jakob Kragstrup, Ebba Holme Hansen, Nicky Britten, and Alicja Malgorzata Oltarzewska, 2004. European Social Survey Round 2 Module Proposal. Health and Care Seeking in a Changing Europe. Bergen: europeansocialsurvey.org/docs/round2/questionnaire/ESS2_kooiker_proposal.pdf.

Kopp, Maria, Árpád Skrabski, János Réthelyi, Ichiro Kawachi, and Nancy E. Adler, 2004. 'SelfRated Health, Subjective Social Status, and Middle-Aged Mortality in a Changing Society.' Behavioral Medicine 30(2): 65-72. doi: 10.3200/BMED.30.2.65-72.

Kraus, Michael W., Nancy E. Adler, and Teh-Way David Chen, 2013. 'Is the Association of Subjective SES and Self-Rated Health Confounded by Negative Mood? An Experimental Approach.' Health Psychology 32(2): 138-145. doi: 10.1037/a0027343.

Kraus, Michael W. and Dacher Keltner, 2009. 'Signs of Socioeconomic Status. A Thin-Slicing Approach.' Psychological Science 20(1): 99-106. doi: 10.1111/j.1467-9280.2008.02251.x.

Kreft, Ita and Jan De Leeuw, 1998. Introducing Multilevel Modeling. London: Sage. doi: $10.4135 / 9781849209366$.

Krieger, Nancy, Jarvis T. Chen, Brent A. Coull, Jason Beckfield, Mathew V. Kiang, and Pamela D. Waterman, 2014. 'Jim Crow and Premature Mortality among the US Black and White Population, 1960-2009. An Age-Period-Cohort Analysis.' Epidemiology 25(4): 494504. doi: 10.1097/ede.0000000000000104.

Krieger, Nancy, David R. Williams, and N. E. Moss, 1997. 'Measuring Social Class in US Public Health Research. Concepts, Methodologies, and Guidelines.' Annual Review of Public Health 18: 341-378. doi: 10.1146/annurev.publhealth.18.1.341.

Kuh, Diana and Yoav Ben-Shlomo, 2004. A Life Course Approach to Chronic Disease Epidemiology. Oxford: Oxford University Press, 2nd edition. doi: 10.1093/acprof:oso/9780198578154.001.0001.

Kunst, Anton E., David A. Leon, Feikje Groenhof, and Johan P. Mackenbach, 1998. 'Occupational Class and Cause-Specific Mortality in Middle Aged Men in Eleven European Countries. Comparison of Population-Based Studies.' BMJ 316(7145): 1636-1642. 10.1136/bmj.316.7145.1636. 
Lahelma, Eero, 2001. 'Health and Social Stratification.' In Cockerham, William C., ed., The Blackwell Companion to Medical Sociology, pp. 64-93. Oxford: Blackwell. doi: 10.1002/9780470996447.ch4.

Lancee, Bram and Herman G. Van de Werfhorst, 2012. 'Income Inequality and Participation. A Comparison of 24 European Countries.' Social Science Research 41(5): 1166-1178. doi: 10.1016/j.ssresearch.2012.04.005.

Lantz, Paula M., James S. House, Richard P. Mero, and David R. Williams, 2005. 'Stress, Life Events, and Socioeconomic Disparities in Health. Results from the Americans' Changing Lives Study.' Journal of Health and Social Behavior 46(3): 274-288. doi: $10.1177 / 002214650504600305$.

Lantz, Paula M., John W. Lynch, James S. House, James M. Lepkowski, Richard P. Mero, Marc A. Musick, and David R. Williams, 2001. 'Socioeconomic Disparities in Health Change in a Longitudinal Study of US Adults. The Role of Health-Risk Behaviors.' Social Science and Medicine 53(1): 29-40. doi: 10.1016/S0277-9536(00)00319-1.

Layte, Richard, 2012. 'The Association Between Income Inequality and Mental Health. Testing Status Anxiety, Social Capital, and Neo-Materialist Explanations.' European Sociological Review 28(4): 498-511. doi: 10.1093/esr/jcr012.

Layte, Richard and Christopher T. Whelan, 2009. 'Explaining Social Class Inequalities in Smoking. The Role of Education, Self-Efficacy, and Deprivation.' European Sociological Review 25(4): 399-410. doi: 10.1093/esr/jcn022.

— 2014 . 'Who Feels Inferior? A Test of the Status Anxiety Hypothesis of Social Inequalities in Health.' European Sociological Review 30(4): 525-535. doi: 10.1093/esr/jcu057.

Lenski, Gerhard E., 1954. 'Status Crystallization. A Non-Vertical Dimension of Social Status.' American Sociological Review 19(4): 405-413. doi: 10.2307/2087459.

Liberatos, Penny, Bruce G. Link, and Jennifer L. Kelsey, 1988. 'The Measurement of Social Class in Epidemiology.' Epidemiologic Reviews 10: 87-121.

Lindemann, Kristina and Ellu Saar, 2014. 'Contextual Effects on Subjective Social Position. Evidence from European Countries.' International Journal of Comparative Sociology 55(1): 3-23. doi: 10.1177/0020715214527101.

Lleras-Muney, Adriana, 2005. 'The Relationship Between Education and Adult Mortality in the United States.' Review of Economic Studies 72(1): 189-221. doi: 10.1111/0034-6527.00329.

Lorant, Vincent, Denise Deliège, William W. Eaton, A. Robert, P. Philippot, and Marc Ansseau, 2003. 'Socioeconomic Inequalities in Depression. A Meta-Analysis.' American Journal of Epidemiology 157(2): 98-112. doi: 10.1093/aje/kwf182.

Lutfey, Karen and Jeremy Freese, 2005. 'Toward Some Fundamentals of Fundamental Causality. Socioeconomic Status and Health in the Routine Clinic Visit for Diabetes.' American Journal of Sociology 110(5): 1326-1372. doi: 10.1086/428914. 
Lynch, John W., George Davey Smith, Sam Harper, Marianne Hillemeier, Nancy Ross, George A. Kaplan, and Michael Wolfson, 2004. 'Is Income Inequality a Determinant of Population Health? Part 1. A Systematic Review.' Milbank Quarterly 82(1): 5-99. doi: 10.1111/j.0887378X.2004.00302.x.

Lynch, John W., George Davey Smith, George A. Kaplan, and James S. House, 2000. 'Income Inequality and Mortality. Importance to Health of Individual Income, Psychosocial Environment, or Material Conditions.' BMJ 320(7243): 1200-1204. doi: 10.1136/bmj.320.7243.1200.

Macintyre, Sally, 1997. 'The Black Report and Beyond. What Are the Issues?' Social Science and Medicine 44(6): 723-745. doi: 10.1016/S0277-9536(96)00183-9.

Mackenbach, Johan P., 2012. 'The Persistence of Health Inequalities in Modern Welfare States. The Explanation of a Paradox.' Social Science and Medicine 75(4): 761-769. doi: 10.1016/j.socscimed.2012.02.031.

Mackenbach, Johan P., Marina Karanikolos, and Martin McKee, 2013. 'The Unequal Health of Europeans. Successes and Failures of Policies.' Lancet 381(9872): 1125-1134. doi: 10.1016/S0140-6736(12)62082-0.

Mackenbach, Johan P., Ivana Kulhánová, Matthias Bopp, Patrick Deboosere, Terje A. Eikemo, Rasmus Hoffmann, Margarete C. Kulik, Mall Leinsalu, Pekka Martikainen, Gwenn Menvielle, Enrique Regidor, Bogdan Wojtyniak, Olof Östergren, Olle Lundberg, and the EURO-GBD-SE Consortium, 2015a. 'Variations in the Relation between Education and Cause-Specific Mortality in 19 European Populations. A Test of the "Fundamental Causes" Theory of Social Inequalities in Health.' Social Science and Medicine 127: 51-62. doi: 10.1016/j.socscimed.2014.05.021.

Mackenbach, Johan P., Ivana Kulhánová, Gwenn Menvielle, Matthias Bopp, Carme Borrell, Giuseppe Costa, Patrick Deboosere, Santiago Esnaola, Ramune Kalediene, Katalin Kovacs, Mall Leinsalu, Pekka Martikainen, Enrique Regidor, Maica Rodriguez-Sanz, Bjørn Heine Strand, Rasmus Hoffmann, Terje A. Eikemo, Olof Östergren, Olle Lundberg, Eurothine consortium, and the EURO-GBD-SE Consortium, 2015b. 'Trends in Inequalities in Premature Mortality. A Study of 3.2 Million Deaths in 13 European Countries.' Journal of Epidemiology and Community Health 69(73): 207-217. doi: 10.1136/jech-2014-204319.

Mackenbach, Johan P. and Anton E. Kunst, 1997. 'Measuring the Magnitude of Socio-Economic Inequalities in Health. An Overview of Available Measures Illustrated with Two Examples from Europe.' Social Science and Medicine 44(6): 757-771. doi: 10.1016/S0277-9536(96)00073-1.

Mackenbach, Johan P., Pekka Martikainen, Caspar W. N. Looman, Jetty A. A. Dalstra, Anton E. Kunst, Eero Lahelma, and members of the SEdHA working group, 2005. 'The Shape of the Relationship between Income and Self-Assessed Health. An International Study.' International Journal of Epidemiology 34(2): 286-293. doi: 10.1093/ije/dyh338.

Mackenbach, Johan P., Irina Stirbu, Albert-Jan R. Roskam, Maartje M. Schaap, Gwenn Menvielle, Mall Leinsalu, and Anton E. Kunst, 2008. 'Socioeconomic Inequalities in Health in 22 European Countries.' New England Journal of Medicine 358: 2468-2481. doi: 10.1056/NEJMsa0707519. 
Marmot, Michael G., 2004. The Status Syndrome. How Social Standing Affects Our Health and Longevity. New York: Holt.

Marmot, Michael G., Geoffrey Rose, Michael J. Shipley, and Peter J. Hamilton, 1978. 'Employment Grade and Coronary Heart Disease in British Civil Servants.' Journal of Epidemiology and Community Health 32(4): 244-249. doi: 10.1136/jech.32.4.244.

Marmot, Michael G., Michael J. Shipley, and Geoffrey Rose, 1984. 'Inequalities in Death. Specific Explanations of a General Pattern?' Lancet 323(8384): 1003-1006. doi: 10.1016/S0140$6736(84) 92337-7$.

Marx, Karl, 1975. 'Economic and Philosophic Manuscripts of 1844.' In Marx-Engels Collected Works. Volume 3: 1843-1844, pp. 229-346. New York: International Publishers.

— 1976. 'The Poverty of Philosophy.' In Marx-Engels Collected Works. Volume 6: 1845-1848, pp. 105-212. New York: International Publishers.

Masters, Ryan K., Robert A. Hummer, and Daniel A. Powers, 2012. 'Educational Differences in US Adult Mortality. A Cohort Perspective.' American Sociological Review 77(4): 548-572. doi: $10.1177 / 0003122412451019$.

Mau, Steffen and Roland Verwiebe, 2010. European Societies. Mapping Structure and Change. Bristol: Policy.

McDonough, Peggy, Diana Worts, and Amanda Sacker, 2010. 'Socioeconomic Inequalities in Health Dynamics. A Comparison of Britain and the United States.' Social Science and Medicine 70(2): 251-260. doi: 10.1016/j.socscimed.2009.10.001.

McEwen, Bruce S., 1998. 'Stress, Adaptation, and Disease. Allostasis and Allostatic Load.' Annals of the New York Academy of Sciences 840(1): 33-44. doi: 10.1111/j.17496632.1998.tb09546.x.

McKeown, Thomas, 1979. The Role of Medicine. Dream, Mirage, or Nemesis? Oxford: Blackwell.

McPherson, Miller, Lynn Smith-Lovin, and Matthew E. Brashears, 2006. 'Social Isolation in America. Changes in Core Discussion Networks over Two Decades.' American Sociological Review 71(3): 353-375. doi: 10.1177/000312240607100301.

Meadows, Sarah O., Sara S. McLanahan, and Jeanne Brooks-Gunn, 2008. 'Stability and Change in Family Structure and Maternal Health Trajectories.' American Sociological Review 73: 314-334. doi: 10.1177/000312240807300207.

Mills, Melinda and Patrick Präg, 2014. Gender Inequalities in the School-to-Work Transition in Europe. Brussels: European Commission DG Justice. doi: 10.2838/54302.

Mills, Melinda, Patrick Präg, Flavia Tsang, Katia Begall, James Derbyshire, Laura Kohle, Céline Miani, and Stijn Hoorens, 2014a. Use of Childcare Services in the EU Member States and Progress Towards the Barcelona Targets. Brussels: European Commission DG Justice. doi: $10.2838 / 54302$. 
Mills, Melinda, Flavia Tsang, Patrick Präg, Kai Ruggeri, Céline Miani, and Stijn Hoorens, 2014b. Gender Equality in the Workforce. Reconciling Work, Private, and Family Life in Europe. Brussels: European Commission DG Justice. doi: 10.2838/54302.

Mills, Melinda, Gerhard G. van de Bunt, and Jeanne de Bruijn, 2006. 'Comparative Research. Persistent Problems and Promising Solutions.' International Sociology 21(5): 619-631. doi: $10.1177 / 0268580906067833$.

Mirowsky, John and Catherine E. Ross, 2003. Education, Social Status, and Health. New York: de Gruyter.

Murray, Christopher J. L., Sandeep C. Kulkarni, and Majid Ezzati, 2005. 'Eight Americas. New Perspectives on US Health Disparities.' American Journal of Preventive Medicine 29(5, Supplement 1): 4-10. doi: 10.1016/j.amepre.2005.07.031.

Murray, Christopher J. L., Sandeep C. Kulkarni, Catherine Michaud, Niels Tomijima, Maria T. Bulzacchelli, Terrell J. Iandiorio, and Majid Ezzati, 2006. 'Eight Americas. Investigating Mortality Disparities across Races, Counties, and Race-Counties in the United States.' PLoS Medicine 3(9): e260. doi: 10.1371/journal.pmed.0030260.

Mussweiler, Thomas, 2003. 'Comparison Processes in Social Judgment. Mechanisms and Consequences.' Psychological Review 110(3): 472-489. doi: 10.1037/0033-295X.110.3.472.

Navarro, Vicente, Carles Muntaner, Carme Borrell, Joan Benach, Águeda Quiroga, Maica Rodríguez-Sanz, Núria Vergés, and M. Isabel Pasarín, 2006. 'Politics and Health Outcomes.' Lancet 368(9540): 1033-1037. doi: 10.1016/S0140-6736(06)69341-0.

Neckermann, Kathryn M. and Florencia Torche, 2007. 'Inequality. Causes and Consequences.' Annual Review of Sociology 33: 335-57. doi: 10.1146/annurev.soc.33.040406.131755.

Nettleton, Sarah, 2006. The Sociology of Health and Illness. Cambridge: Polity, 2nd edition.

Neuberger, Julia, 2000. 'The Educated Patient. New Challenges for the Medical Profession.' Journal of Internal Medicine 247(1): 6-10. doi: 10.1046/j.1365-2796.2000.00624.x.

Nobles, Jenna, Miranda Ritterman Weintraub, and Nancy E. Adler, 2013. 'Subjective Socioeconomic Status and Health. Relationships Reconsidered.' Social Science and Medicine 82(13): 58-66. doi: 10.1016/j.socscimed.2013.01.021.

O’Connor, Annette M., Carol L. Bennett, Dawn Stacey, Michael Barry, Nananda F. Col, Karen B. Eden, Vikki A. Entwistle, Valerie Fiset, Margaret Holmes-Rovner, Sara Khangura, Hilary Llewellyn-Thomas, and David Rovner, 2009. Decision Aids for People Facing Health Treatment or Screening Decisions (Review). London: Cochrane Collaboration.

OECD, 2011. OECD I-Library. Paris: oecd-ilibrary.org.

Olafsdottir, Sigrun, 2007. 'Fundamental Causes of Health Disparities. Stratification, the Welfare State, and Health in the United States and Iceland.' Journal of Health and Social Behavior 48(3): 239-253. doi: 10.1177/002214650704800303. 
Olafsdottir, Sigrun and Jason Beckfield, 2011. 'Health and the Social Rights of Citizenship. Integrating Welfare State Theory and Medical Sociology.' In Pescosolido, Bernice A., Jack K. Martin, Jane D. McLeod, and Anne Rogers, eds., Handbook of the Sociology of Health, Illness, and Healing, pp. 101-115. New York: Springer. doi: 10.1007/978-1-4419-7261-3_6.

Olafsdottir, Sigrun, Jason Beckfield, and Elyas Bakhtiari, 2013. 'Contextualizing Disparities. The Case for Comparative Research on Social Inequalities in Health.' Research in the Sociology of Health Care 31: 299-317. doi: 10.1108/S0275-4959(2013)0000031015.

Olshansky, S. Jay, Toni Antonucci, Lisa Berkman, Robert H. Binstock, Axel Börsch-Supan, John T. Cacioppo, Bruce A. Carnes, Laura L. Carstensen, Linda P. Fried, Dana P. Goldman, James Jackson, Martin Kohli, John Rother, Yuhui Zheng, and John Rowe, 2012. 'Differences in Life Expectancy Due to Race and Educational Differences Are Widening, and Many May Not Catch Up.' Health Affairs 31(8): 1803-1813. doi: 10.1377/hlthaff.2011.0746.

Ong, Lucille M. L., Hanneke C. J. M. De Haes, A. M. Hoos, and Frits B. Lammes, 1995. 'Doctor-Patient Communication. A Review of the Literature.' Social Science and Medicine 40(7): 903-918. doi: 10.1016/0277-9536(94)00155-M.

Operario, Don, Nancy E. Adler, and David R. Williams, 2004. 'Subjective Social Status. Reliability and Predictive Utility for Global Health.' Psychology and Health 19(2): 237-246. doi: 10.1080/08870440310001638098.

Ormel, Johan, Siegwart Lindenberg, Nardi Steverink, and Michael Vonkorrf, 1997. 'Quality of Life and Social Production Functions. A Framework for Understanding Health Effects.' Social Science and Medicine 45(7): 1051-1063. doi: 10.1016/S0277-9536(97)00032-4.

Ostrove, Joan M., Nancy E. Adler, Miriam Kuppermann, and A. Eugenes Washington, 2000. 'Objective and Subjective Assessments of Socioeconomic Status and Their Relationship to Self-Rated Health in an Ethnically Diverse Sample of Pregnant Women.' Health Psychology 19(6): 613-618. doi: 10.1037/0278-6133.19.6.613.

Pampel, Fred C., Patrick M. Krueger, and Justin T. Denney, 2010. 'Socioeconomic Disparities in Health Behaviors.' Annual Review of Sociology 36: 349-370. doi: 10.1146/annurev.soc.012809.102529.

Parsons, Talcott, 1951. The Social System. New York: Free Press.

Paterson, Iain and Ken Judge, 2002. 'Equality of Access to Health Care.' In Mackenbach, Johan P. and Marijntje Bakker, eds., Reducing Inequalities in Health. A European Perspective, pp. 169-187. London: Routledge. doi: 10.4324/9780203167281.

Pavalko, Eliza K. and Jennifer Caputo, 2013. 'Social Inequality and Health Across the Life Course.' American Behavioral Scientist 57(8): 1040-1056. doi: 10.1177/0002764213487344.

Peck, B. Mitchell and Sonya Conner, 2011. 'Talking with Me or Talking at Me? The Impact of Status Characteristics on Doctor-Patient Interaction.' Sociological Perspectives 54(4): 547567. doi: $10.1525 /$ sop.2011.54.4.547. 
Peter, Sascha, Patrick Präg, and Lea Ellwardt, 2014. 'Does Income Inequality Really Erode Social Cohesion? Fixed-Effects Evidence from Europe, 2002-2012.' Paper presented at the RC 28 Spring Meeting in Budapest and the BIGSSS International Conference in Bremen.

Phelan, Jo C., Bruce G. Link, and Parisa Tehranifar, 2010. 'Social Conditions as Fundamental Causes of Health Inequalities. Theory, Evidence, and Policy Implications.' Journal of Health and Social Behavior 51(Supplement): S28-S40. doi: 10.1177/0022146510383498.

Pickett, Kate E. and Richard G. Wilkinson, 2015. 'Income Inequality and Health. A Causal Review.' Social Science and Medicine 128: 316-326. doi: 10.1016/j.socscimed.2014.12.031.

Pop, Ioana, Erik Van Ingen, and Wim Van Oorschot, 2013. 'Inequality, Wealth, and Health. Is Decreasing Income Inequality the Key to Create Healthier Societies?' Social Indicators Research 113(3): 1025-1043. doi: 10.1007/s11205-012-0125-6.

Präg, Patrick, Melinda Mills, and Rafael Wittek, 2014. 'Income and Income Inequality as Social Determinants of Health. Do Social Comparisons Play a Role?' European Sociological Review 30(2): 218-229. doi: 10.1093/esr/jct035.

Präg, Patrick, Maria das Dores Guerreiro, Jouko Nätti, Michael Brookes, and Laura den Dulk, 2011. 'Quality of Work and Quality of Life of Service Sector Workers. Cross-National Variations in Eight European Countries.' In Bäck-Wiklund, Margareta, Tanja Van der Lippe, Laura Den Dulk, and Anneke Van Doorne-Huiskes, eds., Quality of Work and Life. Theory, Practice, and Policy, pp. 77-94. Basingstoke: Palgrave Macmillan. doi: 10.1057/9780230299443_5.

Präg, Patrick, Steffen Mau, and Roland Verwiebe, 2010. 'Quality of Life.' In Mau, Steffen and Roland Verwiebe, eds., European Societies. Mapping Structure and Change, pp. 225-260. Bristol: Policy.

Präg, Patrick and Melinda Mills, 2014. Family-Related Working Schedule Flexibility across Europe. Cambridge: Rand Corporation. doi: 10.2838/54302.

Präg, Patrick, Melinda Mills, Maria Letizia Tanturri, Christiaan Monden, and Gilles Pison, 2015. The Demographic Consequences of Assisted Reproductive Technologies. Deliverable D4.6 of 'Families and Societies'.

Präg, Patrick, Melinda Mills, and Rafael Wittek, 2016. 'Subjective Socio-Economic Status and Health in Cross-National Comparison.' Social Science and Medicine 149: 84-92. doi: 10.1016/j.socscimed.2015.11.044.

Präg, Patrick and S.V. Subramanian, 2017. 'Educational Inequalities in Self-Rated Health across US States and European Countries.' International Journal of Public Health 62(6): 709-716. doi: 10.1007/s00038-017-0981-6.

Präg, Patrick, Rafael Wittek, and Melinda Mills, 2017. 'The Educational Gradient in Self-Rated Health in Europe. Does the Doctor-Patient Relationship Make a Difference?' Acta Sociologica doi: $10.1177 / 0001699316670715$.

Quon, Elizabeth C. and Jennifer J. McGrath, 2014. 'Subjective Socioeconomic Status and Adolescent Health. A Meta-Analysis.' Health Psychology 33(5): 433-447. doi: 10.1037/a0033716. 
Radloff, Lenore Sawyer, 1977. 'The CES-D Scale. A Self-Report Depression Scale for Research in the General Population.' Applied Psychological Measurement 1(3): 385-401. doi: $10.1177 / 014662167700100306$.

Rechel, Bernd, Bayard Roberts, Erica Richardson, Sergey Shishkin, Vladimir M. Shkolnikov, David A. Leon, Martin Bobak, Marina Karanikolos, and Martin McKee, 2013. 'Health and Health Systems in the Commonwealth of Independent States.' Lancet 381(9872): 1145-1155. doi: 10.1016/S0140-6736(12)62084-4.

Reitzel, Lorraine R., Jennifer I. Vidrine, Yisheng Li, Patricia D. Mullen, Mary M. Velasquez, Paul M. Cinciripini, Ludmila Cofta-Woerpel, Anthony Greisinger, and David W. Wetter, 2007. 'The Influence of Subjective Social Status on Vulnerability to Postpartum Smoking Among Young Pregnant Women.' American Journal of Public Health 97(8): 1476-1482. doi: 10.2105/AJPH.2006.101295.

Rodgers, Gerry B., 1979. 'Income and Inequality as Determinants of Mortality. An International Cross-Section Analysis.' Population Studies 33(2): 343-351. doi: 10.1080/00324728.1979.10410449.

Ross, Catherine E. and John Mirowsky, 1995. 'Does Employment Affect Health?' Journal of Health and Social Behavior 36(3): 230-243. doi: 10.2307/2137340.

— , 2002. 'Family Relationships, Social Support, and Subjective Life Expectancy.' Journal of Health and Social Behavior 43(4): 469-489. doi: 10.2307/3090238.

Ross, Catherine E. and Chia-Ling Wu, 1996. 'Education, Age, and the Cumulative Advantage in Health.' Journal of Health and Social Behavior 37(1): 104-120. doi: 10.2307/2137234.

Runciman, Walter, 2009. 'How Messy It All Is.' London Review of Books 31(20): 3-6.

Ryan, Richard M. and Edward L. Deci, 2000. 'Self-Determination Theory and the Facilitation of Intrinsic Motivation, Social Development, and Well-Being.' American Psychologist 55(1): 68-78. doi: 10.1037/0003-066X.55.1.68.

Sacker, Amanda, Richard D. Wiggins, Mel Bartley, and Peggy McDonough, 2007. 'Self-Rated Health Trajectories in the United States and the United Kingdom. A Comparative Study.' American Journal of Public Health 97(5): 812-818. doi: 10.2105/AJPH.2006.092320.

Sakurai, Keiko, Norito Kawakami, Kazue Yamaoka, Hirono Ishikawa, and Hideki Hashimoto, 2010. 'The Impact of Subjective and Objective Social Status on Psychological Distress among Men and Women in Japan.' Social Science and Medicine 70(11): 1832-1839. doi: 10.1016/j.socscimed.2010.01.019.

Sapolsky, Robert M., 2004. Why Zebras Don't Get Ulcers. The Acclaimed Guide to Stress, Stress-Related Diseases, and Coping. New York: Holt.

_, 2005. 'The Influence of Social Hierarchy on Primate Health.' Science 308(5722): 648-652. doi: $10.1126 /$ science.1106477. 
Schneider, Simone M. and Jürgen Schupp, 2014. 'Individual Differences in Social Comparison and its Consequences for Life Satisfaction. Introducing a Short Scale of the IowaNetherlands Comparison Orientation Measure.' Social Indicators Research 115(2): 767-789. doi: $10.1007 / \mathrm{s} 11205-012-0227-1$.

Schnittker, Jason and Jane D. McLeod, 2005. 'The Social Psychology of Health Disparities.' Annual Review of Sociology 31: 75-103. doi: 10.1146/annurev.soc.30.012703.110622.

Seeman, Melvin, Sharon Merkin, Arun Karlamangla, Brandon Koretz, and Teresa Seeman, 2014. 'Social Status and Biological Dysregulation. The "Status Syndrome" and Allostatic Load.' Social Science and Medicine 118: 143-151. doi: 10.1016/j.socscimed.2014.08.002.

Semyonov, Moshe, Noah Lewin-Epstein, and Dina Maskileyson, 2013. 'Where Wealth Matters More for Health. The Wealth-Health Gradient in 16 Countries.' Social Science and Medicine 81: 10-17. doi: 10.1016/j.socscimed.2013.01.010.

Shively, Carol A., 2000. 'Social Status, Stress, and Health in Female Monkeys.' In Tarlov, Alvin R. and Richard F. St. Peter, eds., The Society and Population Health Reader. A State and Community Perspective, pp. 278-289. New York: New Press.

Singh-Manoux, Archana, Nancy E. Adler, and Michael G. Marmot, 2003. 'Subjective Social Status. Its Determinants and Its Association with Measures of Ill-Health in the Whitehall II Study.' Social Science and Medicine 56(1): 1321-1333. doi: 10.1016/S0277-9536(02)00131-4.

Singh-Manoux, Archana, Michael G. Marmot, and Nancy E. Adler, 2005. 'Does Subjective Social Status Predict Health and Change in Health Status Better Than Objective Status?' Psychosomatic Medicine 67(6): 855-861. doi: 10.1097/01.psy.0000188434.52941.a0.

Snijders, Tom and Roel Boskers, 2012. Multilevel Analysis. An Introduction to Basic and Advanced Multilevel Modeling. London: Sage, 2nd edition.

Solt, Frederick, 2009. 'Standardizing the World Income Inequality Database.' Social Science Quarterly 90(2): 231-242. doi: 10.1111/j.1540-6237.2009.00614.x.

Stavropoulou, Charitini, 2011. 'Non-Adherence to Medication and Doctor-Patient Relationship. Evidence from a European Survey.' Patient Education and Counseling 83(1): 7-13. doi: 10.1016/j.pec.2010.04.039.

— 2012. 'The Doctor-Patient Relationship. A Review of the Theory and Policy Implications.' In McGuire, Alistair and Joan Costa-Font, eds., The LSE Companion to Health Policy, pp. 314-326. Cheltenham: Edward Elgar. doi: 10.4337/9781781004241.00030.

Steinhart, Brian, 2002. 'Patient Autonomy. Evolution of the Doctor-Patient Relationship.' Haemophilia 8(3): 441-446. doi: 10.1046/j.1365-2516.2002.00614.x.

Stewart, Moira A., 1995a. 'Effective Physician-Patient Communication and Health Outcomes. A Review.' Canadian Medical Association Journal 152(9): 1423-1433.

_ 1995b. 'Studies of Health Outcome and Patient-Centered Communication.' In Stewart, Moira A., Judith Belle Brown, W. Wayne Weston, Ian R. McWhinney, Carol L. McWilliam, and Thomas R. Freeman, eds., Patient-Centered Medicine. Transforming the Clinical Method, pp. 185-190. Thousand Oaks, CA: Sage. 
Stolk, Ronald P., Inge Hutter, and Rafael Wittek, 2009. 'Population Aging Research. A Family of Disciplines.' European Journal of Epidemiology 24(11): 715-718. doi: 10.1007/s10654-0099398-8.

Subramanian, SV, Kelvyn Jones, and Craig Duncan, 2003. 'Multilevel Methods for Public Health Research.' In Kawachi, Ichiro and Lisa F. Berkman, eds., Neighborhoods and Health, pp. 65111. New York: Oxford University Press. doi: 10.1093/acprof:oso/9780195138382.003.0004.

Subramanian, SV, Kelvyn Jones, Afamia Kaddour, and Nancy Krieger, 2009. 'Revisiting Robinson. The Perils of Individualistic and Ecologic Fallacy.' International Journal of Epidemiology 38(2): 342-360. doi: 10.1093/ije/dyn359.

Suls, Jerry, 2003. 'Contributions of Social Comparison to Physical Illness and Well-Being.' In Suls, Jerry and Kenneth A. Wallston, eds., Social Psychological Foundations of Health and Illness, pp. 226-255. Malden, MA: Blackwell. doi: 10.1002/9780470753552.ch9.

Suls, Jerry and Ladd Wheeler, 2000. 'A Selective History of Classic and Neo-Social Comparison Theory.' In Suls, Jerry and Ladd Wheeler, eds., Handbook of Social Comparison. Theory and Research, pp. 1-23. Dordrecht: Kluwer. doi: 10.1007/978-1-4615-4237-7_1.

Tabachnick, Barbara G. and Linda S. Fidell, 2007. Using Multivariate Statistics. Boston, MA: Pearson/Allyn and Bacon.

Thiede, Michael and Stefan Traub, 1997. 'Mutual Influences of Health and Poverty. Evidence from German Panel Data.' Social Science and Medicine 45(6): 867-877. doi: 10.1016/S02779536(96)00427-3.

Thoits, Peggy A., 2010. 'Stress and Health. Major Findings and Policy Implications.' Journal of Health and Social Behavior 51(Supplement): S41-S53. doi: 10.1177/0022146510383498.

— 2011. 'Mechanisms Linking Social Ties and Support to Physical and Mental Health.' Journal of Health and Social Behavior 52(2): 145-161. doi: 10.1177/0022146510395592.

Thorpe, Kenneth E., David H. Howard, and Katya Galactionova, 2007. 'Differences in Disease Prevalence as a Source of the US-European Health Care Spending Gap.' Health Affairs 26(6): w678-w686. doi: 10.1377/hlthaff.26.6.w678.

Torssander, Jenny and Robert Erikson, 2010. 'Stratification and Mortality. A Comparison of Education, Class, Status, and Income.' European Sociological Review 26(4): 465-474. doi: 10.1093/esr/jcp034.

Townsend, Peter and Nick Davidson, 1982. Inequalities in Health. The Black Report. London: Penguin.

Umberson, Debra, Robert Crosnoe, and Corinne Reczek, 2010. 'Social Relationships and Health Behavior Across the Life Course.' Annual Review of Sociology 36: 139-157. doi: 10.1146/annurev-soc-070308-120011.

UNESCO, 2006. ISCED 199\%. International Standard Classification of Education. Montreal: UNESCO Institute for Statistics. 
US Burden of Disease Collaborators, 2013. 'The State of US Health, 1990-2010. Burden of Diseases, Injuries, and Risk Factors.' JAMA 310(6): 591-608. doi: 10.1001/jama.2013.13805.

Van de Velde, Sarah, Piet Bracke, and Katia Levecque, 2010a. 'Gender Differences in Depression in 23 European Countries. Cross-National Variation in the Gender Gap in Depression.' Social Science and Medicine 71(2): 305-313. doi: 10.1016/j.socscimed.2010.03.035.

Van de Velde, Sarah, Piet Bracke, Katia Levecque, and Bart Meuleman, 2010b. 'Gender Differences in Depression in 25 European Countries after Eliminating Measurement Bias in the CES-D 8.' Social Science Research 39(3): 396-404. doi: 10.1016/j.ssresearch.2010.01.002.

Veblen, Thorstein, 1899. The Theory of the Leisure Class. An Economic Study of Institutions. London: George Allen \& Unwin.

Von dem Knesebeck, Olaf, Pablo E. Verde, and Nico Dragano, 2006. 'Education and Health in 22 European Countries.' Social Science and Medicine 63(5): 1344-1351. doi: 10.1016/j.socscimed.2006.03.043.

Weber, Max, 1968. Economy and Society. An Outline of Interpretive Sociology. Berkeley, CA: University of California Press.

Weiner, Saul J., Alan Schwartz, Gunjan Sharma, Amy Binns-Calvey, Naomi Ashley, Brendan Kelly, Amit Dayal, Sonal Patel, Frances M. Weaver, and Ilene Harris, 2013. 'Patient-Centered Decision Making and Health Care Outcomes. An Observational Study.' Annals of Internal Medicine 158(8): 573-580. doi: 10.7326/0003-4819-158-8-201304160-00001.

Wendt, Claus, Lorraine Frisina, and Heinz Rothgang, 2009. 'Healthcare System Types. A Conceptual Framework for Comparison.' Social Policy and Administration 43(1): 70-90. doi: 10.1111/j.1467-9515.2008.00647.x.

Whelan, Christopher T. and Bertrand Maître, 2013. 'Material Deprivation, Economic Stress, and Reference Groups in Europe. An Analysis of EU-SILC 2009.' European Sociological Review 29(6): 1162-1174. doi: 10.1093/esr/jct006.

White, Robert W., 1959. 'Motivation Reconsidered. The Concept of Competence.' Psychological Review 66(5): 297-333. doi: 10.1037/h0040934.

Whitehead, Margaret, 1997. 'Life and Death over the Millennium.' In Drever, Frances and Margaret Whitehead, eds., Health Inequalities. Decennial Supplement, pp. 7-28. London: HMSO.

Wilkinson, Richard G., 1992. 'Income Distribution and Life Expectancy.' BMJ 304(6820): 165-168. doi: 10.1136/bmj.304.6820.165.

- 1996. Unhealthy Societies. The Affictions of Inequality. London and New York: Routledge. doi: $10.4324 / 9780203421680$.

— 1997. 'Health Inequalities. Relative or Absolute Material Standards?' BMJ 314(7080): 591-595. doi: 10.1136/bmj.314.7080.591.

Wilkinson, Richard G. and Kate E. Pickett, 2006. 'Income Inequality and Population Health. A Review and Explanation of the Evidence.' Social Science and Medicine 62(7): 1768-1784. doi: 10.1016/j.socscimed.2005.08.036. 
— , 2008. 'Income Inequality and Socioeconomic Gradients in Mortality.' American Journal of Public Health 98(4): 699-704. doi: 10.2105/AJPH.2007.109637.

—_, 2009. 'Income Inequality and Social Dysfunction.' Annual Review of Sociology 35: 493-511. doi: 10.1146/annurev-soc-070308-115926.

—, 2010. The Spirit Level. Why Equality Is Better for Everyone. London: Penguin, 3rd edition.

Willems, S., S. De Maesschalck, M. Deveugele, A. Derese, and J. De Maeseneer, 2005. 'SocioEconomic Status of the Patient and Doctor-Patient Communication. Does It Make a Difference?' Patient Education and Counseling 56(2): 139-146. doi: 10.1016/j.pec.2004.02.011.

Williams, Geoffrey C., Richard M. Frankel, Thomas L. Campbell, and Edward L. Deci, 2000. 'Research on Relationship-Centered Care and Healthcare Outcomes from the Rochester Biopsychosocial Program. A Self-Determination Theory Integration.' Families, Systems, and Health 18(1): 79-90. doi: 10.1037/h0091854.

Williams, Mark V., Ruth M. Parker, David W. Baker, Nina S. Parikh, Kathryn Pitkin, Wendy C. Coates, and Joanne R. Nurss, 1995. 'Inadequate Functional Health Literacy Among Patients at Two Public Hospitals.' JAMA 274(21): 1677-1682. doi: 10.1001/jama.1995.03530210031026.

Willson, Andrea E., Kim M. Shuey, and Jr. Elder, Glen H., 2007. 'Cumulative Advantage Processes as Mechanisms of Inequality in Life Course Health.' American Journal of Sociology 112(6): 1886-1924. doi: 10.1086/512712.

Wimmer, Andreas and Nina Glick Schiller, 2002. 'Methodological Nationalism and Beyond. Nation-State Building, Migration, and the Social Sciences.' Global Networks 2(4): 301-334. doi: 10.1111/1471-0374.00043.

Wolff, Lisa S., Dolores Acevedo-Garcia, SV Subramanian, Deanne Weber, and Ichiro Kawachi, 2010a. 'Subjective Social Status, a New Measure in Health Disparities Research. Do Race/Ethnicity and Choice of Referent Group Matter?' Journal of Health Psychology 15(4): 560-574. doi: $10.1177 / 1359105309354345$.

Wolff, Lisa S., SV Subramanian, Dolores Acevedo-Garcia, Deanne Weber, and Ichiro Kawachi, 2010b. 'Compared to Whom? Subjective Social Status, Self-Rated Health, and Referent Group Sensitivity in a Diverse US Sample.' Social Science and Medicine 70(12): 2019-2028. doi: 10.1016/j.socscimed.2010.02.033.

Wooldridge, Jeffrey M., 2010. Econometric Analysis of Cross Section and Panel Data. Cambridge, MA: MIT Press, 2nd edition.

Woolf, Steven H. and Laudan Aron, 2013. US Health in International Perspective. Shorter Lives, Poorer Health. Washington, DC: National Academies Press. doi: 10.17226/13497.

World Bank, 2010. World Development Indicators and Global Delevopment Finance. Washington, DC: databank.worldbank.org.

- 2014a. Measuring the Real Size of the World Economy. The Framework, Methodology, and Results of the International Comparison Program (ICP). Washington, DC: World Bank. doi: 10.1596/978-0-8213-9728-2. 
- 2014b. World Development Indicators 2014. Washington, DC: World Bank. doi: 10.1596/978-1-4648-0163-1.

Wright, Caroline E. and Andrew Steptoe, 2005. 'Subjective Socioeconomic Position, Gender, and Cortisol Responses to Waking in an Elderly Population.' Psychoneuroendocrinology 30(6): 582-590. doi: 10.1016/j.psyneuen.2005.01.007.

Wright, Eric Olin, 1997. Class Counts. Comparative Studies in Class Analysis. Cambridge: Cambridge University Press. doi: 10.1017/CBO9780511488917. 



\section{Dutch summary}

In deze studie staat het verband tussen sociale stratificatie en gezondheid centraal. Mensen met een hogere opleiding, een betere baan, een hoger inkomen of hogere status leven langer en gezonder. Niettemin zijn een aantal belangrijke vragen nog niet beantwoord, in het bijzonder de vraag naar de specifieke mechanismen die ten grondslag liggen aan dit verband. Om inzicht te krijgen in deze mechanismen, richt deze studie zich op het veelzijdige karakter van het sociaaleconomische status (SES). Eerder onderzoek heeft aangetoond dat SES niet met slechts één variabele gemeten kan worden, maar dat meerdere dimensies van SES bekeken moeten worden. Niet alleen objectieve indicatoren van sociale stratificatie, zoals opleiding, inkomen of beroepsstatus, maar onderzoek heeft aangetoond dat ook subjectieve dimensies van sociale stratificatie van belang zijn.

Het verband tussen sociale stratificatie en gezondheid is ook op verschillende niveaus belangrijk. Aan de ene kant zijn er veel SES indicatoren die op het niveau van de individu een rol spelen, zoals opleiding, beroepsprestige of subjectief waargenomen SES. Aan de andere kant is SES ook belangrijk op een hoger niveau. Hierbij kan men denken aan de relatie tussen artsen en patiënten of de geaggregeerde inkomensongelijkheid in een land. Deze factoren hebben ook een aantoonbaar effect op de gezondheid.

In dit boek beantwoorden wij enkele onderzoeksvragen op het gebied van sociale verschillen in gezondheid. Hierbij wordt gebruik gemaakt van een cross-nationaal perspectief waarbij zowel objectieve als subjectieve SES indicatoren worden gebruikt.

Voor de empirische analyses wordt er gebruik gemaakt van data op individueel niveau uit verschillende landen, zoals de European Social Survey (ESS), de European Values Study (EVS) of het International Social Survey Program (ISSP). Deze survey data zijn aangevuld met data op nationaal niveau (zoals inkomensongelijkheid) uit verschillende bronnen, o.a. Eurostat, de Wereldbank en de OESO. De gebruikte uitkomstvariabelen zijn de algehele gezondheidstoestand en psychologisch welzijn. Om de hypothesen te toetsen wordt gebruik gemaakt van multi-level regressie modellen. 
In hoofdstuk 2 wordt onderzocht hoe het verband tussen opleiding en subjectieve gezondheid verschilt tussen de Verenigde Staten en Europa. Eerder onderzoek heeft aangetoond dat de gezondheid in de Verenigde Staten slechter is dan in vergelijkbare, geïndustrialiseerde landen. Dit is in strijd met het feit dat de Amerikaanse gezondheidszorg per capita de duurste ter wereld is. Verder blijkt uit eerdere studies dat er ook een grote regionale heterogeniteit binnen de Verenigde Staten is. De empirische analyses combineren wij het grootschalige Behavioral Risk Factor Surveillance System (BRFSS) uit de Verenigde Staten met de European Values Study (EVS). De BRFSS maakt het mogelijk opleidingsverschillen in gezondheid tussen de vijftig deelstaten van de VS te bekijken en de EVS omvat representatieve informatie over de gezondheid van Europeanen op landelijk niveau voor bijna alle Europese landen. De bevindingen tonen aan dat de prevalentie van slechte subjectieve gezondheid en opleidingsverschillen in gezondheid groter zijn in de Verenigde Staten. Maar er zijn ook belangrijke uitzonderingen; sommige deelstaten van de VS vertonen een betere gezondheid dan Europese landen en enkele Oost-Europese landen vertonen een even slechte gezondheid als de VS. Ook als we naar de opleidingsverschillen in gezondheid kijken zijn er enkele, vaak Oost-Europese landen, met verschillen die even groot zijn als in de VS.

In hoofdstuk 3 wordt ingegaan op het verband tussen subjectieve SES en gezondheid vanuit cross-nationaal perspectief. Uit eerder onderzoek blijkt er een robuust verband aanwezig te zijn tussen subjectieve SES en gezondheid. Dit verband blijft ook bestaan als voor objectieve indicatoren van SES (zoals inkomen, educatie en beroepsstatus) wordt gecontroleerd. Verder toont eerder onderzoek aan dat er variatie is in de sterkte van het verband tussen objectieve indicatoren van SES en gezondheid in verschillende landen. Middels data uit 27 landen tonen wij aan dat er in elk land een verband bestaat tussen subjectieve SES en twee indicatoren van gezondheid (subjectieve gezondheid en psychologisch welzijn), zelfs als wij voor de objectieve SES indicatoren opleiding, inkomen, en beroepsstatus controleren. Ook blijkt uit de resultaten dat er een belangrijke mate van variatie is in de sterkte van het verband tussen subjectieve SES en gezondheid tussen de 27 landen. Deze variatie is echter niet afhankelijk van verschillen in inkomensongelijkheid en welvaart tussen landen.

In hoofdstuk 4 staat - naast het verband tussen inkomen en gezondheid - een ander aspect van de relatie tussen sociale stratificatie en gezondheid centraal: het verband tussen inkomensongelijkheid en gezondheid. Deze verbanden zijn vaak verklaard aan de hand van een statusvergelijkingsmechanisme, maar dit mechanisme wordt zelden op een directe manier getoetst. In dit hoofdstuk werken wij dit mechanisme uit en toetsen het ook empirisch via de rol van social vergelijking oriëntaties. Uit eerder onderzoek is gebleken dat mensen verschillen in hun 
geneigdheid zichzelf met anderen te vergelijken. Dus zijn mensen met een sterkere socialevergelijkingsoriëntatie (neiging tot het vergelijken met anderen) sterker beïnvloed door de resultaten van sociale vergelijkingen. In de analyses toetsen wij of sociale vergelijkingen de verbanden tussen inkomen en gezondheid op het individueel niveau en inkomensongelijkheid in een land en gezondheid kunnen verklaren. Er wordt data uit 23 Europese landen gebruikt met twee afhankelijke variabelen (subjectieve gezondheid en psychologisch welzijn) om onze hypotheses te toetsen. Uit onze analyses blijkt dat er een klein negatief effect is van socialevergelijkingsoriëntatie op gezondheid, maar dat het statusvergelijkingsmechanisme het positieve verband tussen inkomen en gezondheid en het negatieve verband tussen inkomensongelijkheid en gezondheid niet kan verklaren. Deze resultaten trekken theorieën van wetenschappers als Wilkinson en Pickett in twijfel, omdat bij hen sociale vergelijking centraal staat voor het verklaren van deze verbanden.

In hoofdstuk 5 wordt het effect van de relatie tussen artsen en patiënten op opleidingsverschillen in gezondheid onderzocht. Eerder onderzoek heeft aangetoond dat relaties tussen artsen en patiënten geëvolueerd zijn van een artsen-gecentreerde, paternalistische aanpak naar een patiënten-gecentreerde, egalitaire aanpak van interactie tussen artsen en hun patiënten. Maar hoe beïnvloedt deze ontwikkeling opleidingsverschillen in gezondheid? Om deze vraag te beantwoorden wordt gebruik gemaakt van data uit 24 Europese landen. Ten eerste toetsen wij of een patiëntengecentreerde aanpak geassocieerd is met een zwakker verband tussen opleiding en gezondheid. Ten tweede wordt onderzocht of de patiënten-gecentreerde dan wel de artsen-gecentreerde aanpak een verschillende invloed heeft op de gezondheid voor mensen met hoge of lage opleiding. Uit de resultaten blijkt dat verschillende aanpakken van de relatie tussen artsen en patiënten niet geassocieerd zijn met opleidingsverschillen in gezondheid. Verder tonen onze analyses aan dat patiënten met een lage opleiding van een egalitaire, patiënten-gecentreerde aanpak profiteren, zodat het algehele gezondheidsniveau onder de bevolking stijgt. 



\section{Curriculum Vitae}

Patrick Präg holds a BA in Social Sciences from the University of Düsseldorf, Germany, and an MA in Criminology from the University of Hamburg, Germany. He obtained a PhD from the Interuniversity Center for Social Science Theory and Methodology (ICS) at the Department of Sociology at the University of Groningen, The Netherlands, with the present dissertation. The research for this dissertation was partially conducted at the Harvard School of Public Health in Boston (Mass.), USA. Since September 2014, Patrick is a research fellow at Nuffield College and a postdoctoral researcher at the Department of Sociology at the University of Oxford, United Kingdom. 



\section{ICS Dissertation Series}

The ICS series presents dissertations of the Interuniversity Center for Social Science Theory and Methodology. Each of these studies aims at integrating explicit theory formation with state-of-the-art empirical research or at the development of advanced methods for empirical research. The ICS was founded in 1986 as a cooperative effort of the universities of Groningen and Utrecht. Since 1992, the ICS expanded to the University of Nijmegen. Most of the projects are financed by the participating universities or by the Netherlands Organization for Scientific Research (NWO). The international composition of the ICS graduate students is mirrored in the increasing international orientation of the projects and thus of the ICS series itself.

1. C. van Liere. (1990). Lastige leerlingen. Een empirisch onderzoek naar sociale oorzaken van probleemgedrag op basisscholen. Amsterdam: Thesis Publishers.

2. Marco H.D. van Leeuwen. (1990). Bijstand in Amsterdam, ca. 1800-1850. Armenzorg als beheersings- en overlevingsstrategie. ICS dissertation, Utrecht.

3. I. Maas. (1990). Deelname aan podiumkunsten via de podia, de media en actieve beoefening. Substitutie of leereffecten? Amsterdam: Thesis Publishers.

4. M.I. Broese van Groenou. (1991). Gescheiden netwerken. De relaties met vrienden en verwanten na echtscheiding. Amsterdam: Thesis Publishers.

5. Jan M.M. van den Bos. (1991). Dutch EC policy making. A model-guided approach to coordination and negotiation. Amsterdam: Thesis Publishers.

6. Karin Sanders. (1991). Vrouwelijke pioniers. Vrouwen en mannen met een 'mannelijke' hogere beroepsopleiding aan het begin van hun loopbaan. Amsterdam: Thesis Publishers.

7. Sjerp de Vries. (1991). Egoism, altruism, and social justice. Theory and experiments on cooperation in social dilemmas. Amsterdam: Thesis Publishers.

8. Ronald S. Batenburg. (1991). Automatisering in bedrijf. Amsterdam: Thesis Publishers.

9. Rudi Wielers. (1991). Selectie en allocatie op de arbeidsmarkt. Een uitwerking voor de informele en geïnstitutionaliseerde kinderopvang. Amsterdam: Thesis Publishers.

10. Gert P. Westert. (1991). Verschillen in ziekenhuisgebruik. ICS dissertation, Groningen. 
11. Hanneke Hermsen. (1992). Votes and policy preferences. Equilibria in party systems. Amsterdam: Thesis Publishers.

12. Cora J.M. Maas. (1992). Probleemleerlingen in het basisonderwijs. Amsterdam: Thesis Publishers.

13. Ed A.W. Boxman. (1992). Contacten en carrière. Een empirisch-theoretisch onderzoek naar de relatie tussen sociale netwerken en arbeidsmarktposities. Amsterdam: Thesis Publishers.

14. Conny G.J. Taes. (1992). Kijken naar banen. Een onderzoek naar de inschatting van arbeidsmarktkansen bij schoolverlaters uit het middelbaar beroepsonderwijs. Amsterdam: Thesis Publishers.

15. Peter van Roozendaal. (1992). Cabinets in multi-party democracies. The effect of dominant and central parties on cabinet composition and durability. Amsterdam: Thesis Publishers.

16. Marcel van Dam. (1992). Regio zonder regie. Verschillen in en effectiviteit van gemeentelijk arbeidsmarktbeleid. Amsterdam: Thesis Publishers.

17. Tanja van der Lippe. (1993). Arbeidsverdeling tussen mannen en vrouwen. Amsterdam: Thesis Publishers.

18. Marc A. Jacobs. (1993). Software: Kopen of kopiëren? Een sociaal-wetenschappelijk onderzoek onder PC-gebruikers. Amsterdam: Thesis Publishers.

19. Peter van der Meer. (1993). Verdringing op de Nederlandse arbeidsmarkt. Sectoren sekseverschillen. Amsterdam: Thesis Publishers.

20. Gerbert Kraaykamp. (1993). Over lezen gesproken. Een studie naar sociale differentiatie in leesgedrag. Amsterdam: Thesis Publishers.

21. Evelien Zeggelink. (1993). Strangers into friends. The evolution of friendship networks using an individual oriented modeling approach. Amsterdam: Thesis Publishers.

22. Jaco Berveling. (1994). Het stempel op de besluitvorming. Macht, invloed en besluitvorming op twee Amsterdamse beleidsterreinen. Amsterdam: Thesis Publishers.

23. Wim Bernasco. (1994). Coupled careers. The effects of spouse's resources on success at work. Amsterdam: Thesis Publishers.

24. Liset van Dijk. (1994). Choices in child care. The distribution of child care among mothers, fathers and non-parental care providers. Amsterdam: Thesis Publishers.

25. Jos de Haan. (1994). Research groups in Dutch sociology. Amsterdam: Thesis Publishers.

26. K. Boahene. (1995). Innovation adoption as a socio-economic process. The case of the Ghanaian cocoa industry. Amsterdam: Thesis Publishers.

27. Paul E.M. Ligthart. (1995). Solidarity in economic transactions. An experimental study of framing effects in bargaining and contracting. Amsterdam: Thesis Publishers.

28. Roger Th. A.J. Leenders. (1995). Structure and influence. Statistical models for the dynamics of actor attributes, network structure, and their interdependence. Amsterdam: Thesis Publishers. 
29. Beate Völker. (1995). Should auld acquaintance be forgot ...? Institutions of communism, the transition to capitalism and personal networks: The case of East Germany. Amsterdam: Thesis Publishers.

30. A. Cancrinus-Matthijsse. (1995). Tussen hulpverlening en ondernemerschap. Beroepsuitoefening en taakopvattingen van openbare apothekers in een aantal WestEuropese landen. Amsterdam: Thesis Publishers.

31. Nardi Steverink. (1996). Zo lang mogelijk zelfstandig. Naar een verklaring van verschillen in oriëntatie ten aanzien van opname in een verzorgingstehuis onder fysiek kwetsbare ouderen. Amsterdam: Thesis Publishers.

32. Ellen Lindeman. (1996). Participatie in vrijwilligerswerk. Amsterdam: Thesis Publishers.

33. Chris Snijders. (1996). Trust and commitments. Amsterdam: Thesis Publishers.

34. Koos Postma. (1996). Changing prejudice in Hungary. A study on the collapse of state socialism and its impact on prejudice against gypsies and Jews. Amsterdam: Thesis Publishers.

35. Jooske T. van Busschbach. (1996). Uit het oog, uit het hart? Stabiliteit en verandering in persoonlijke relaties. Amsterdam: Thesis Publishers.

36. René Torenvlied. (1996). Besluiten in uitvoering. Theorieën over beleidsuitvoering modelmatig getoetst op sociale vernieuwing in drie gemeenten. Amsterdam: Thesis Publishers.

37. Andreas Flache. (1996). The double edge of networks. An analysis of the effect of informal networks on cooperation in social dilemmas. Amsterdam: Thesis Publishers.

38. Kees van Veen. (1997). Inside an internal labor market: Formal rules, flexibility and career lines in a Dutch manufacturing company. Amsterdam: Thesis Publishers.

39. Lucienne van Eijk. (1997). Activity and well-being in the elderly. Amsterdam: Thesis Publishers.

40. Róbert Gál. (1997). Unreliability. Contract discipline and contract governance under economic transition. Amsterdam: Thesis Publishers.

41. Anne-Geerte van de Goor. (1997). Effects of regulation on disability duration. ICS dissertation, Utrecht.

42. Boris Blumberg. (1997). Das Management von Technologiekooperationen. Partnersuche und Verhandlungen mit dem Partner aus empirisch-theoretischer Perspektive. ICS dissertation, Utrecht.

43. Marijke von Bergh. (1997). Loopbanen van oudere werknemers. Amsterdam: Thesis Publishers.

44. Anna Petra Nieboer. (1997). Life events and well-being: A prospective study on changes in well-being of elderly people due to a serious illness event or death of the spouse. Amsterdam: Thesis Publishers.

45. Jacques Niehof. (1997). Resources and social reproduction: The effects of cultural and material resources on educational and occupational careers in industrial nations at the end of the twentieth century. ICS dissertation, Nijmegen. 
46. Ariana Need. (1997). The kindred vote. Individual and family effects of social class and religion on electoral change in the Netherlands, 1956-1994. ICS dissertation, Nijmegen.

47. Jim Allen. (1997). Sector composition and the effect of education on wages: An international comparison. Amsterdam: Thesis Publishers.

48. Jack B.F. Hutten. (1998). Workload and provision of care in general practice. An empirical study of the relation between workload of Dutch general practitioners and the content and quality of their care. ICS dissertation, Utrecht.

49. Per B. Kropp. (1998). Berufserfolg im Transformationsprozeß, Eine theoretischempirische Studie über die Gewinner und Verlierer der Wende in Ostdeutschland. ICS dissertation, Utrecht.

50. Maarten H.J. Wolbers. (1998). Diploma-inflatie en verdringing op de arbeidsmarkt. Een studie naar ontwikkelingen in de opbrengsten van diploma's in Nederland. ICS dissertation, Nijmegen.

51. Wilma Smeenk. (1998). Opportunity and marriage. The impact of individual resources and marriage market structure on first marriage timing and partner choice in the Netherlands. ICS dissertation, Nijmegen.

52. Marinus Spreen. (1999). Sampling personal network structures: Statistical inference in ego-graphs. ICS dissertation, Groningen.

53. Vincent Buskens. (1999). Social networks and trust. ICS dissertation, Utrecht.

54. Susanne Rijken. (1999). Educational expansion and status attainment. A crossnational and over-time comparison. ICS dissertation, Utrecht.

55. Mérove Gijsberts. (1999). The legitimation of inequality in state-socialist and market societies, 1987-1996. ICS dissertation, Utrecht.

56. Gerhard G. Van de Bunt. (1999). Friends by choice. An actor-oriented statistical network model for friendship networks through time. ICS dissertation, Groningen.

57. Robert Thomson. (1999). The party mandate: Election pledges and government actions in the Netherlands, 1986-1998. Amsterdam: Thela Thesis.

58. Corine Baarda. (1999). Politieke besluiten en boeren beslissingen. Het draagvlak van het mestbeleid tot 2000. ICS dissertation, Groningen.

59. Rafael Wittek. (1999). Interdependence and informal control in organizations. ICS dissertation, Groningen.

60. Diane Payne. (1999). Policy making in the European Union: An analysis of the impact of the reform of the structural funds in Ireland. ICS dissertation, Groningen.

61. René Veenstra. (1999). Leerlingen - klassen - scholen. Prestaties en vorderingen van leerlingen in het voortgezet onderwijs. Amsterdam: Thela Thesis.

62. Marjolein Achterkamp. (1999). Influence strategies in collective decision making. A comparison of two models. ICS dissertation, Groningen.

63. Peter Mühlau. (2000). The governance of the employment relation. A relational signaling perspective. ICS dissertation, Groningen.

64. Agnes Akkerman. (2000). Verdeelde vakbeweging en stakingen. Concurrentie om leden. ICS dissertation, Groningen. 
65. Sandra van Thiel. (2000). Quangocratization: Trends, causes and consequences. ICS dissertation, Utrecht.

66. Rudi Turksema. (2000). Supply of day care. ICS dissertation, Utrecht.

67. Sylvia E. Korupp (2000). Mothers and the process of social stratification. ICS dissertation, Utrecht.

68. Bernard A. Nijstad (2000). How the group affects the mind: Effects of communication in idea generating groups. ICS dissertation, Utrecht.

69. Inge F. de Wolf (2000). Opleidingsspecialisatie en arbeidsmarktsucces van sociale wetenschappers. ICS dissertation, Utrecht.

70. Jan Kratzer (2001). Communication and performance: An empirical study in innovation teams. ICS dissertation, Groningen.

71. Madelon Kroneman (2001). Healthcare systems and hospital bed use. ICS/NIVEL dissertation, Utrecht.

72. Herman van de Werfhorst (2001). Field of study and social inequality. Four types of educational resources in the process of stratification in the Netherlands. ICS dissertation, Nijmegen.

73. Tamás Bartus (2001). Social capital and earnings inequalities. The role of informal job search in Hungary. ICS dissertation, Groningen.

74. Hester Moerbeek (2001). Friends and foes in the occupational career. The influence of sweet and sour social capital on the labour market. ICS dissertation, Nijmegen.

75. Marcel van Assen (2001). Essays on actor perspectives in exchange networks and social dilemmas. ICS dissertation, Groningen.

76. Inge Sieben (2001). Sibling similarities and social stratification. The impact of family background across countries and cohorts. ICS dissertation, Nijmegen.

77. Alinda van Bruggen (2001). Individual production of social well-being. An exploratory study. ICS dissertation, Groningen.

78. Marcel Coenders (2001). Nationalistic attitudes and ethnic exclusionism in a comparative perspective: An empirical study of attitudes toward the country and ethnic immigrants in 22 countries. ICS dissertation, Nijmegen.

79. Marcel Lubbers (2001). Exclusionistic electorates. Extreme right-wing voting in Western Europe. ICS dissertation, Nijmegen.

80. Uwe Matzat (2001). Social networks and cooperation in electronic communities. A theoretical-empirical analysis of academic communication and internet discussion groups. ICS dissertation, Groningen.

81. Jacques P.G. Janssen (2002). Do opposites attract divorce? Dimensions of mixed marriage and the risk of divorce in the Netherlands. ICS dissertation, Nijmegen.

82. Miranda Jansen (2002). Waardenoriëntaties en partnerrelaties. Een panelstudie naar wederzijdse invloeden. ICS dissertation, Utrecht.

83. Anne Rigt Poortman (2002). Socioeconomic causes and consequences of divorce. ICS dissertation, Utrecht.

84. Alexander Gattig (2002). Intertemporal decision making. ICS dissertation, Groningen. 
85. Gerrit Rooks (2002). Contract en conflict: Strategisch management van inkooptransacties. ICS dissertation, Utrecht.

86. Károly Takács (2002). Social networks and intergroup conflict. ICS dissertation, Groningen.

87. Thomas Gautschi (2002). Trust and exchange, effects of temporal embeddedness and network embeddedness on providing and dividing a surplus. ICS dissertation, Utrecht.

88. Hilde Bras (2002). Zeeuwse meiden. Dienen in de levensloop van vrouwen, ca. 1850-1950. Amsterdam: Aksant Academic Publishers.

89. Merijn Rengers (2002). Economic lives of artists. Studies into careers and the labour market in the cultural sector. ICS dissertation, Utrecht.

90. Annelies Kassenberg (2002). Wat scholieren bindt. Sociale gemeenschap in scholen. ICS dissertation, Groningen

91. Marc Verboord (2003). Moet de meester dalen of de leerling klimmen? De invloed van literatuuronderwijs en ouders op het lezen van boeken tussen 1975 en 2000. ICS dissertation, Utrecht.

92. Marcel van Egmond (2003). Rain falls on all of us (but some manage to get more wet than others): Political context and electoral participation. ICS dissertation, Nijmegen.

93. Justine Horgan (2003). High performance human resource management in Ireland and the Netherlands: Adoption and effectiveness. ICS dissertation, Groningen.

94. Corine Hoeben (2003). LETS' be a community. Community in local exchange trading systems. ICS dissertation, Groningen.

95. Christian Steglich (2003). The framing of decision situations. Automatic goal selection and rational goal pursuit. ICS dissertation, Groningen.

96. Johan van Wilsem (2003). Crime and context. The impact of individual, neighborhood, city and country characteristics on victimization. ICS dissertation, Nijmegen.

97. Christiaan Monden (2003). Education, inequality and health. The impact of partners and life course. ICS dissertation, Nijmegen.

98. Evelyn Hello (2003). Educational attainment and ethnic attitudes. How to explain their relationship. ICS dissertation, Nijmegen.

99. Marnix Croes en Peter Tammes (2004). Gif laten wij niet voortbestaan. Een onderzoek naar de overlevingskansen van joden in de Nederlandse gemeenten, 19401945. Amsterdam: Aksant Academic Publishers.

100. Ineke Nagel (2004). Cultuurdeelname in de levensloop. ICS dissertation, Utrecht.

101. Marieke van der Wal (2004). Competencies to participate in life. Measurement and the impact of school. ICS dissertation, Groningen.

102. Vivian Meertens (2004). Depressive symptoms in the general population: A multifactorial social approach. ICS dissertation, Nijmegen.

103. Hanneke Schuurmans (2004). Promoting well-being in frail elderly people: Theory and intervention. ICS dissertation, Groningen.

104. Javier Arregui (2004). Negotiation in legislative decision-making in the European Union. ICS dissertation, Groningen. 
105. Tamar Fischer (2004). Parental divorce, conflict and resources. The effects on children's behaviour problems, socioeconomic attainment, and transitions in the demographic career. ICS dissertation, Nijmegen.

106. René Bekkers (2004). Giving and volunteering in the Netherlands: Sociological and psychological perspectives. ICS dissertation, Utrecht.

107. Renée van der Hulst (2004). Gender differences in workplace authority: An empirical study on social networks. ICS dissertation, Groningen.

108. Rita Smaniotto (2004). 'You scratch my back and I scratch yours' versus 'Love thy neighbour': Two proximate mechanisms of reciprocal altruism. ICS dissertation, Groningen.

109. Maurice Gesthuizen (2004). The life-course of the low-educated in the Netherlands: Social and economic risks. ICS dissertation, Nijmegen.

110. Carlijne Philips (2005). Vakantiegemeenschappen. Kwalitatief en kwantitatief onderzoek naar gelegenheid- en refreshergemeenschap tijdens de vakantie. ICS dissertation, Groningen.

111. Esther de Ruijter (2005). Household outsourcing. ICS dissertation, Utrecht.

112. Frank van Tubergen (2005). The integration of immigrants in cross-national perspective: Origin, destination, and community effects. ICS dissertation, Utrecht.

113. Ferry Koster (2005). For the time being. Accounting for inconclusive findings concerning the effects of temporary employment relationships on solidary behavior of employees. ICS dissertation, Groningen.

114. Carolien Klein Haarhuis (2005). Promoting anti-corruption reforms. Evaluating the implementation of a World Bank anti-corruption program in seven African countries (1999-2001). ICS dissertation, Utrecht.

115. Martin van der Gaag (2005). Measurement of individual social capital. ICS dissertation, Groningen.

116. Johan Hansen (2005). Shaping careers of men and women in organizational contexts. ICS dissertation, Utrecht.

117. Davide Barrera (2005). Trust in embedded settings. ICS dissertation, Utrecht.

118. Mattijs Lambooij (2005). Promoting cooperation. Studies into the effects of long-term and short-term rewards on cooperation of employees. ICS dissertation, Utrecht.

119. Lotte Vermeij (2006). What's cooking? Cultural boundaries among Dutch teenagers of different ethnic origins in the context of school. ICS dissertation, Utrecht.

120. Mathilde Strating (2006). Facing the challenge of rheumatoid arthritis. A 13-year prospective study among patients and cross-sectional study among their partners. ICS dissertation, Groningen.

121. Jannes de Vries (2006). Measurement error in family background variables: The bias in the intergenerational transmission of status, cultural consumption, party preference, and religiosity. ICS dissertation, Nijmegen.

122. Stefan Thau (2006). Workplace deviance: Four studies on employee motives and self-regulation. ICS dissertation, Groningen. 
123. Mirjam Plantinga (2006). Employee motivation and employee performance in child care. The effects of the introduction of market forces on employees in the Dutch child-care sector. ICS dissertation, Groningen.

124. Helga de Valk (2006). Pathways into adulthood. A comparative study on family life transitions among migrant and Dutch youth. ICS dissertation, Utrecht.

125. Henrike Elzen (2006). Self-management for chronically ill older people. ICS dissertation, Groningen.

126. Ayşe Güveli (2007). New social classes within the service class in the Netherlands and Britain. Adjusting the EGP class schema for the technocrats and the social and cultural specialists. ICS dissertation, Nijmegen.

127. Willem-Jan Verhoeven (2007). Income attainment in post-communist societies. ICS dissertation, Utrecht.

128. Marieke Voorpostel (2007). Sibling support: The exchange of help among brothers and sisters in the Netherlands. ICS dissertation, Utrecht.

129. Jacob Dijkstra (2007). The effects of externalities on partner choice and payoffs in exchange networks. ICS dissertation, Groningen.

130. Patricia van Echtelt (2007). Time-greedy employment relationships: Four studies on the time claims of post-Fordist work. ICS dissertation, Groningen.

131. Sonja Vogt (2007). Heterogeneity in social dilemmas: The case of social support. ICS dissertation, Utrecht.

132. Michael Schweinberger (2007). Statistical methods for studying the evolution of networks and behavior. ICS dissertation, Groningen.

133. István Back (2007). Commitment and evolution: Connecting emotion and reason in long-term relationships. ICS dissertation, Groningen.

134. Ruben van Gaalen (2007). Solidarity and ambivalence in parent-child relationships. ICS dissertation, Utrecht.

135. Jan Reitsma (2007). Religiosity and solidarity: Dimensions and relationships disentangled and tested. ICS dissertation, Nijmegen.

136. Jan Kornelis Dijkstra (2007) Status and affection among (pre)adolescents and their relation with antisocial and prosocial behavior. ICS dissertation, Groningen.

137. Wouter van Gils (2007). Full-time working couples in the Netherlands. Causes and consequences. ICS dissertation, Nijmegen.

138. Djamila Schans (2007). Ethnic diversity in intergenerational solidarity. ICS dissertation, Utrecht.

139. Ruud van der Meulen (2007). Brug over woelig water: Lidmaatschap van sportverenigingen, vriendschappen, kennissenkringen en veralgemeend vertrouwen. ICS dissertation, Nijmegen.

140. Andrea Knecht (2008). Friendship selection and friends' influence. Dynamics of networks and actor attributes in early adolescence. ICS dissertation, Utrecht.

141. Ingrid Doorten (2008). The division of unpaid work in the household: A stubborn pattern? ICS dissertation, Utrecht.

142. Stijn Ruiter (2008). Association in context and association as context: Causes and consequences of voluntary association involvement. ICS dissertation, Nijmegen. 
143. Janneke Joly (2008). People on our minds: When humanized contexts activate social norms. ICS dissertation, Groningen.

144. Margreet Frieling (2008). 'Joint production' als motor voor actief burgerschap in de buurt. ICS-dissertion, Groningen.

145. Ellen Verbakel (2008). The partner as resource or restriction? Labour market careers of husbands and wives and the consequences for inequality between couples. ICS dissertation, Nijmegen.

146. Gijs van Houten (2008). Beleidsuitvoering in gelaagde stelsels. De doorwerking van aanbevelingen van de Stichting van de Arbeid in het CAO-overleg. ICS dissertation, Utrecht.

147. Eva Jaspers (2008). Intolerance over time: Macro and micro level questions on attitudes towards euthanasia, homosexuality and ethnic minorities. ICS dissertation, Nijmegen.

148. Gijs Weijters (2008). Youth delinquency in Dutch cities and schools: A multilevel approach. ICS dissertation, Nijmegen.

149. Jessica Pass (2009). The self in social rejection. ICS dissertation, Groningen.

150. Gerald Mollenhorst (2009). Networks in contexts. How meeting opportunities affect personal relationships. ICS dissertation, Utrecht.

151. Tom van der Meer (2009). States of freely associating citizens: Comparative studies into the impact of state institutions on social, civic and political participation. ICS dissertation, Nijmegen.

152. Manuela Vieth (2009). Commitments and reciprocity in trust situations. Experimental studies on obligation, indignation, and self-consistency. ICS dissertation, Utrecht.

153. Rense Corten (2009). Co-evolution of social networks and behavior in social dilemmas: Theoretical and empirical perspectives. ICS dissertation, Utrecht.

154. Arieke J. Rijken (2009). Happy families, high fertility? Childbearing choices in the context of family and partner relationships. ICS dissertation, Utrecht.

155. Jochem Tolsma (2009). Ethnic hostility among ethnic majority and minority groups in the Netherlands. An investigation into the impact of social mobility experiences, the local living environment and educational attainment on ethnic hostility. ICS dissertation, Nijmegen.

156. Freek Bucx (2009). Linked lives: Young adults' life course and relations with parents. ICS dissertation, Utrecht.

157. Philip Wotschack (2009). Household governance and time allocation. Four studies on the combination of work and care. ICS dissertation, Groningen.

158. Nienke Moor (2009). Explaining worldwide religious diversity. The relationship between subsistence technologies and ideas about the unknown in pre-industrial and (post-)industrial societies. ICS dissertation, Nijmegen.

159. Lieke ten Brummelhuis (2009). Family matters at work. Depleting and enriching effects of employees' family lives on work outcomes. ICS dissertation, Utrecht.

160. Renske Keizer (2010). Remaining childless. Causes and consequences from a life course perspective. ICS dissertation, Utrecht. 
161. Miranda Sentse (2010). Bridging contexts: The interplay between family, child, and peers in explaining problem behavior in early adolescence. ICS dissertation, Groningen.

162. Nicole Tieben (2010). Transitions, tracks and transformations. Social inequality in transitions into, through and out of secondary education in The Netherlands for cohorts born between 1914 and 1985. ICS dissertation, Nijmegen.

163. Birgit Pauksztat (2010). Speaking up in organizations: Four studies on employee voice. ICS dissertation, Groningen.

164. Richard Zijdeman (2010). Status attainment in The Netherlands, 1811-1941. Spatial and temporal variation before and during industrialization. ICS dissertation, Utrecht.

165. Rianne Kloosterman (2010). Social background and children's educational careers. The primary and secondary effects of social background over transitions and over time in the Netherlands. ICS dissertation, Nijmegen.

166. Olav Aarts (2010). Religious diversity and religious involvement. A study of religious markets in Western societies at the end of the twentieth century. ICS dissertation, Nijmegen.

167. Stephanie Wiesmann (2010). 24/7 negotiation in couples transition to parenthood. ICS dissertation, Utrecht.

168. Borja Martinovic (2010). Interethnic contacts: A dynamic analysis of interaction between immigrants and natives in Western countries. ICS dissertation, Utrecht.

169. Anne Roeters (2010). Family life under pressure? Parents' paid work and the quantity and quality of parent-child and family time. ICS dissertation, Utrecht.

170. Jelle Sijtsema (2010). Adolescent aggressive behavior: Status and stimulation goals in relation to the peer context. ICS dissertation, Groningen.

171. Kees Keizer (2010). The spreading of disorder. ICS dissertation, Groningen.

172. Michael Mäs (2010). The diversity puzzle: Explaining clustering and polarization of opinions. ICS dissertation, Groningen.

173. Marie-Louise Damen (2010). Cultuurdeelname en CKV. Studies naar effecten van kunsteducatie op de cultuurdeelname van leerlingen tijdens en na het voortgezet onderwijs. ICS dissertation, Utrecht.

174. Marieke van de Rakt (2011). Two generations of crime: The intergenerational transmission of convictions over the life course. ICS dissertation, Nijmegen.

175. Willem Huijnk (2011). Family life and ethnic attitudes: The role of the family for attitudes towards intermarriage and acculturation among minority and majority groups. ICS dissertation, Utrecht.

176. Tim Huijts (2011). Social ties and health in Europe: Individual associations, crossnational variations, and contextual explanations. ICS dissertation, Nijmegen.

177. Wouter Steenbeek (2011). Social and physical disorder: How community, business presence and entrepreneurs influence disorder in Dutch neighborhoods. ICS dissertation, Utrecht.

178. Miranda Vervoort (2011). Living together apart? Ethnic concentration in the neighborhood and ethnic minorities' social contacts and language practices. ICS dissertation, Utrecht. 
179. Agnieszka Kanas (2011). The economic performance of immigrants. The role of human and social capital. ICS dissertation, Utrecht.

180. Lea Ellwardt (2011). Gossip in organizations. A social network study. ICS dissertation, Groningen.

181. Annemarije Oosterwaal (2011). The gap between decision and implementation: Decision making, delegation and compliance in governmental and organizational settings. ICS dissertation, Utrecht.

182. Natascha Notten (2011). Parents and the media. Causes and consequences of parental media socialization. ICS dissertation, Nijmegen.

183. Tobias Stark (2011). Integration in schools. A process perspective on students' interethnic attitudes and interpersonal relationships. ICS dissertation, Groningen.

184. Giedo Jansen (2011). Social cleavages and political choices. Large-scale comparisons of social class, religion and voting behavior in Western democracies. ICS dissertation, Nijmegen.

185. Ruud van der Horst (2011). Network effects on treatment results in a closed forensic psychiatric setting. ICS dissertation, Groningen.

186. Mark Levels (2011). Abortion laws in European countries between 1960 and 2010. Legislative developments and their consequences for women's reproductive decisionmaking. ICS dissertation, Nijmegen.

187. Marieke van Londen (2012). Exclusion of ethnic minorities in the Netherlands. The effects of individual and situational characteristics on opposition to ethnic policy and ethnically mixed neighborhoods. ICS dissertation, Nijmegen.

188. Sigrid M. Mohnen (2012). Neighborhood context and health: How neighborhood social capital affects individual health. ICS dissertation, Utrecht.

189. Asya Zhelyazkova (2012). Compliance under controversy: Analysis of the transposition of European directives and their provisions. ICS dissertation, Utrecht.

190. Valeska Korff (2012). Between cause and control: Management in a humanitarian organization. ICS dissertation, Groningen.

191. Maike Gieling (2012). Dealing with diversity: Adolescents' support for civil liberties and immigrant rights. ICS dissertation, Utrecht.

192. Katya Ivanova (2012). From parents to partners: The impact of family on romantic relationships in adolescence and emerging adulthood. ICS dissertation, Groningen.

193. Jelmer Schalk (2012). The performance of public corporate actors: Essays on effects of institutional and network embeddedness in supranational, national, and local collaborative contexts. ICS dissertation, Utrecht.

194. Alona Labun (2012). Social networks and informal power in organizations. ICS dissertation, Groningen.

195. Michał Bojanowski (2012). Essays on social network formation in heterogeneous populations: Models, methods, and empirical analyses. ICS dissertation, Utrecht.

196. Anca Minescu (2012). Relative group position and intergroup attitudes in Russia. ICS dissertation, Utrecht.

197. Marieke van Schellen (2012). Marriage and crime over the life course. The criminal careers of convicts and their spouses. ICS dissertation, Utrecht. 
198. Mieke Maliepaard (2012). Religious trends and social integration: Muslim minorities in the Netherlands. ICS dissertation, Utrecht.

199. Fransje Smits (2012). Turks and Moroccans in the Low Countries around the year 2000: Determinants of religiosity, trend in religiosity and determinants of the trend. ICS dissertation, Nijmegen.

200. Roderick Sluiter (2012). The diffusion of morality policies among Western European countries between 1960 and 2010: A comparison of temporal and spatial diffusion patterns of six morality and eleven non-morality policies. ICS dissertation, Nijmegen.

201. Nicoletta Balbo (2012). Family, friends, and fertility. ICS dissertation, Groningen.

202. Anke Munniksma (2013). Crossing ethnic boundaries: Parental resistance to and consequences of adolescents' cross-ethnic peer relations. ICS dissertation, Groningen.

203. Anja Abendroth (2013). Working women in Europe. How the country, workplace, and family context matter. ICS dissertation, Utrecht.

204. Katia Begall (2013). Occupational hazard? The relationship between working conditions and fertility. ICS dissertation, Groningen.

205. Hidde Bekhuis (2013). The popularity of domestic cultural products: Cross-national differences and the relation to globalization. ICS dissertation, Utrecht.

206. Lieselotte Blommaert (2013). Are Joris and Renske more employable than Rashid and Samira? A study on the prevalence and sources of ethnic discrimination in recruitment in the Netherlands using experimental and survey data. ICS dissertation, Utrecht.

207. Wiebke Schulz (2013). Careers of men and women in the 19th and 20th centuries. ICS dissertation, Utrecht.

208. Ozan Aksoy (2013). Essays on social preferences and beliefs in non-embedded social dilemmas. ICS dissertation, Utrecht.

209. Dominik Morbitzer (2013). Limited farsightedness in network formation. ICS dissertation, Utrecht.

210. Thomas de Vroome (2013). Earning your place: The relation between immigrants' economic and psychological integration in the Netherlands. ICS dissertation, Utrecht.

211. Marloes de Lange (2013). Causes and consequences of employment flexibility among young people: Recent developments in the Netherlands and Europe. ICS dissertation, Nijmegen.

212. Roza Meuleman (2014). Consuming the nation. Domestic cultural consumption: Its stratification and relation with nationalist attitudes. ICS dissertation, Utrecht.

213. Esther Havekes (2014). Putting interethnic attitudes in context. The relationship between neighborhood characteristics, interethnic attitudes and residential behavior. ICS dissertation, Utrecht.

214. Zoltán Lippényi (2014). Transitions toward an open society? Intergenerational occupational mobility in Hungary in the 19th and 20th centuries. ICS dissertation, Utrecht. 
215. Anouk Smeekes (2014). The presence of the past: Historical rooting of national identity and current group dynamics. ICS dissertation, Utrecht.

216. Michael Savelkoul (2014). Ethnic diversity and social capital. Testing underlying explanations derived from conflict and contact theories in Europe and the United States. ICS dissertation, Nijmegen.

217. Martijn Hogerbrugge (2014). Misfortune and family: How negative events, family ties, and lives are linked. ICS dissertation, Utrecht.

218. Gina Potarca (2014). Modern love. Comparative insights in online dating preferences and assortative mating. ICS dissertation, Groningen.

219. Mariska van der Horst (2014). Gender, aspirations, and achievements: Relating work and family aspirations to occupational outcomes. ICS dissertation, Utrecht.

220. Gijs Huitsing (2014). A social network perspective on bullying. ICS dissertation, Groningen.

221. Thomas Kowalewski (2015). Personal growth in organizational contexts. ICS dissertation, Groningen.

222. Manu Muñoz-Herrera (2015). The impact of individual differences on network relations: Social exclusion and inequality in productive exchange and coordination games. ICS dissertation, Groningen.

223. Tim Immerzeel (2015). Voting for a change. The democratic lure of populist radical right parties in voting behavior. ICS dissertation, Utrecht.

224. Fernando Nieto Morales (2015). The control imperative: Studies on reorganization in the public and private sectors. ICS dissertation, Groningen.

225. Jellie Sierksma (2015). Bounded helping: How morality and intergroup relations shape children's reasoning about helping. ICS dissertation, Utrecht.

226. Tinka Veldhuis (2015). Captivated by fear. An evaluation of terrorism detention policy. ICS dissertation, Groningen.

227. Miranda Visser (2015). Loyality in humanity. Turnover among expatriate humanitarian aid workers. ICS dissertation, Groningen.

228. Sarah Westphal (2015). Are the kids alright? Essays on postdivorce residence arrangements and children's well-being. ICS dissertation, Utrecht.

229. Britta Rüschoff (2015). Peers in careers: Peer relationships in the transition from school to work. ICS dissertation, Groningen.

230. Nynke van Miltenburg (2015). Cooperation under peer sanctioning institutions: Collective decisions, noise, and endogenous implementation. ICS dissertation, Utrecht.

231. Antonie Knigge (2015). Sources of sibling similarity. Status attainment in the Netherlands during modernization. ICS dissertation, Utrecht.

232. Sanne Smith (2015). Ethnic segregation in friendship networks. Studies of its determinants in English, German, Dutch, and Swedish school classes. ICS dissertation, Utrecht.

233. Patrick Präg (2015). Social stratification and health. Four essays on the social determinants of health and wellbeing. ICS dissertation, Groningen. 
The relationship between socioeconomic status, health, and wellbeing has puzzled researchers for decades. Individuals with higher education, a better job, or higher status, live longer and healthier. The four essays in this book offer a fresh view on crucial aspects of this relationship. Drawing on data from more than fifty countries, the multidimensional nature of socioeconomic status is explored, not only covering objective indicators of socioeconomic status-education, income, occupational prestige- - but also individuals' subjective perceptions of socioeconomic status and status differentials between doctors and patients. Furthermore, the essays analyze the multilevel nature of social stratification, comparing differences in the strength of the relationship between education and health across countries and regions, as well as investigating the pathways by which income inequality affects health.

Patrick Präg conducted the present study as part of his doctoral degree at the Interuniversity Center for Social Science Theory and Methodology (ICS) and the Department of Sociology of the University of Groningen. Parts of the research were conducted during a research visit at the Harvard T.H. Chan School of Public Health. He is currently a postdoctoral researcher at the Department of Sociology at the University of Oxford and a research fellow at Nuffield College.

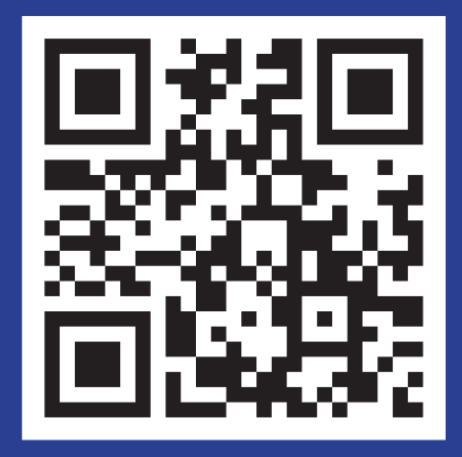

